\title{
PARTIAL REGULARITY OF ALMOST MINIMIZING RECTIFIABLE $G$ CHAINS IN HILBERT SPACE
}

\author{
THIERRY DE PAUW AND ROGER ZÜST
}

\begin{abstract}
We adapt to an infinite dimensional ambient space E.R. Reifenberg's epiperimetric inequality and a quantitative version of D. Preiss' second moments computations to establish that the set of regular points of an almost mass minimizing rectifiable $G$ chain in $\ell_{2}$ is dense in its support, whenever the group $G$ of coefficients is so that $\{\|g\|: g \in G\}$ is discrete and closed.
\end{abstract}

\section{Contents}

1. Introduction

2. Preliminary results for chains in a Hilbert space 8

2.1. Grassmannian and Hausdorff distance 9

2.2. Jacobians in Hilbert space 12

2.3. Chains, Slicing and tangent planes 12

2.4. Excess over a plane 18

2.5. Quadratic forms associated to a chain 21

3. Reifenberg's epiperimetric inequality for a polyhedral cone 25

3.1. Assumptions and preliminaries 26

3.2. Mass estimate of the averaged cone 28

3.3. Plane selection via a quadratic form 44

3.4. A competitor better than the cone 50

4. Moments computations and good approximations by planes 57

4.1. Nearly monotonic, almost monotonic and epiperimetric chains $\quad 57$

4.2. Moments computations $\quad 59$

4.3. Uniform closeness to planes 68

5. Regularity of almost minimizer $\quad 72$

5.1. Polyhedral approximation and a differential inequality 74

5.2. Upper excess bounds and Reifenberg flatness $\quad 80$

5.3. Main regularity results 84

6. Examples and Counterexamples $\quad 91$

References $\quad 94$

The first author was partially supported by the Science and Technology Commission of Shanghai (No. 18dz2271000). The second author was partially supported by grant P2EZP2_148732 of the Swiss National Science Foundation. Both authors were partially supported by project ANR-12-BS01-0014-01 Geometrya. 


\section{INTRODUCTION}

Let $(X,|\cdot|)$ be a separable Hilbert space, and $(G,\|\cdot\|)$ be a complete normed Abelian group, and $m$ be a nonnegative integer. We consider $m$ dimensional rectifiable $G$ chains in $X, T \in \mathscr{R}_{m}(X ; G)$, introduced in [13]. Such $T$ is associated with an $m$ dimensional rectifiable Borel subset $M \subset X$ and a Borel measurable $G$ orientation $\mathbf{g}(x)$ corresponding to $\mathscr{H}^{m}$ almost every $x \in M$. Specifically at almost every $x \in M$ where $M$ admits an approximate $m$ dimensional tangent space $W \subset X, \mathbf{g}(x)$ is an equivalence class $(\xi, g)$ where $\xi$ is an orientation of $W$ and $g \in G$. Here $(\xi, g)$ and $\left(\xi^{\prime}, g^{\prime}\right)$ are equivalent if either they are equal or $\xi$ and $\xi^{\prime}$ are opposite orientations of $W$ and $g=-g^{\prime}$. We set $\|\mathbf{g}\|=\|(\xi, g)\|=\|g\|$. For the data consisting of $M$ and $\mathbf{g}$ to correspond to a member $T \in \mathscr{R}_{m}(X ; G)$ we also require that its mass be finite:

$$
\mathbf{M}(T)=\int_{M}\|\mathbf{g}(x)\| d \mathscr{H}^{m}(x)<\infty .
$$

In [13] $m$ dimensional rectifiable $G$ chains are understood as members of the larger groups $\mathscr{F}_{m}(X ; G)$ of $m$ dimensional flat $G$ chains. This allows for introducing the standard tools of Geometric Measure Theory : Boundary Operator; PushForward by Lipschitzian Mappings; Restriction; Slicing by Lipschitzian Mappings; Support of a Chain; Convergence in Flat Norm; Constancy Theorem, see [14]; Approximation by Polyhedral $G$ chains, see [10]. We will use all of these in the present paper.

The support $\operatorname{spt}(T)$ of $T \in \mathscr{R}_{m}(X ; G)$ consists of those $x \in X$ such that $T\llcorner\mathbf{B}(x, r) \neq 0$ for all $r>0$. Without further restriction it may be the case that $\operatorname{spt}(T)=X$; this can be achieved for $T$ consisting of a mass convergent series of properly chosen $G$ oriented circles whose collection of centers is dense. We say $a \in \operatorname{spt}(T) \backslash \operatorname{spt}(\partial T)$ is a regular point of $T$ whenever there exists a neighborhood $U$ of $a$ in $X$ such that $\operatorname{spt}(T) \cap U$ is an embedded $m$ dimensional Hölder continuously differentiable submanifold of $X$. In this situation there exists a possibly smaller neighborhood $V \subset U$ of $a$ with the following property. There are $C \geq 0, \alpha>0$ and $0<\delta \leq \infty$ such that for every $x \in V$, every $0<r<\min \{\delta$, $\operatorname{dist}(x, \operatorname{spt}(\partial T))\}$, and every $\bar{S} \in \mathscr{R}_{m}(X ; G)$, if $\operatorname{spt}(S) \subset \mathbf{B}(x, r)$ and $\partial S=0$, then

$$
\mathbf{M}\left(T\llcorner\mathbf{B}(x, r)) \leq\left(1+C r^{\alpha}\right) \mathbf{M}(T\llcorner\mathbf{B}(x, r)+S) .\right.
$$

If $T$ verifies the property stated in the last sentence, we say $T$ is $\left(\mathbf{M}, C r^{\alpha}, \delta\right)$ minimizing in $V$. If the specific $C, \alpha$ and $\delta$ are irrelevant we simply say $T$ is almost mass minimizing in $V$. This class of geometric variational objects was introduced, and their regularity studied, by F.J. Almgren [2] in the framework of subsets of $X=\ell_{2}^{n}$ rather than chains. Still in a finite dimensional ambient space, E. Bombieri [8] adapted Almgren's definition to rectifiable $\mathbb{Z}$ chains, essentially as that given above, and studied their regularity following [2].

Examples of $\left(\mathbf{M}, C r^{\alpha}, \delta\right)$ minimizing chains $T \in \mathscr{R}_{m}(X ; G)$ in $V$ encompass the case when $C=0, \delta=\infty$ and $V=X \backslash \operatorname{spt}(\partial T)$. These include mass minimizing $G$ chains in the sense that $\mathbf{M}(T)=\inf \left\{\mathbf{M}(S): S \in \mathscr{R}_{m}(X ; G)\right.$ and $\left.\partial S=\partial T\right\}$. If $a$ is a regular point of such mass minimizing $T$ and $W_{a}$ is the tangent space of $\operatorname{spt}(T)$ at $a$ then $\operatorname{spt}(T)$ is, in a neighborhood of $a$, the (translated) graph of a smooth $f: W_{a} \rightarrow W_{a}^{\perp}$ (with $f(0)=0$ ). Furthermore, near the origin, $f$ satisfies the minimal surface equation in case $m+1=\operatorname{dim} X$, the minimal surface system in case $m+1<\operatorname{dim} X<\infty$, and a corresponding infinite dimensional system of Partial 
Differential Equations in case $\operatorname{dim} X=\infty$. Since $D f(0)=0$ (as $W_{a}$ is tangent to $\operatorname{spt}(T)$ at $a$ ) the so-called blow-up $\hat{f}$ of $f$, i.e. the weak limit in the Hilbert Sobolev space of properly rescaled and renormalized versions of $f$, is harmonic : $\triangle\langle\hat{f}, e\rangle=0$ whenever $e$ is a unit vector in $W_{a}^{\perp}$. A weak version of this observation applied to Lipschitzian maps $f$ that approximate the support of $T$ near points $a$ that verify additional "closeness to flat" assumptions, is at the heart of many proofs of Regularity Theorems for either minimizers of mass or stationary chains. This technique goes back to E. De Giorgi [9]. However in case $T$ is $\left(\mathbf{M}, C r^{\alpha}, \delta\right)$ minimizing, $a$ is a regular point of $\operatorname{spt}(T)$ and the latter is, near $a$, the graph of some Hölder continuously differentiable $f: W_{a} \rightarrow W_{a}^{\perp}$, then $f$ need not solve any Partial Differential Equation whatsoever, as indeed the graph of any such $f$ has the almost mass minimizing property. Our main result is as follows.

1.1. Theorem. Assume $G$ is such that $\{\|g\|: g \in G\}$ is discrete and closed and $T \in \mathscr{R}_{m}(X ; G)$ is almost mass minimizing in an open set $V \subset X \backslash \operatorname{spt}(\partial T)$. It follows that there exists a relatively open set $U \subset \operatorname{spt}(T) \cap V$ which is an embedded $m$ dimensional Hölder continuously differentiable submanifold of $X$, and which is dense in $\operatorname{spt}(T) \cap V$. If one further assumes that $\|g\|=1$ for all $g \in G \backslash\left\{0_{G}\right\}$ then $\mathscr{H}^{m}(\operatorname{spt}(T) \cap V \backslash U)=0$.

We now review relevant earlier results in this vein.

- The case of minimizers in the finite dimensional setting. Here $T \in$ $\mathscr{R}_{m}\left(\ell_{2}^{n} ; G\right)$ is mass minimizing. If $m+1=n$ and $G=\mathbb{Z}$, this is E. De Giorgi's Theorem [9] established in the framework of oriented frontiers. In case $m$ is arbitrary the result has been established by E.R. Reifenberg $[20,21]$ in a different setting than ours. E.R. Reifenberg [19] considers some compact groups of coefficients, he considers sets rather than rectifiable chains and the boundary conditions are expressed by means of Čech homology groups, finally his sets minimize size rather than mass, i.e. Hausdorff measure not weighted by coefficients norm. His method differs from the analysis of blow-up set forth by E. De Giorgi and has inspired the present paper. F.J. Almgren establishes the result in the framework of rectifiable chains minimizing the integral of some elliptic integrands, [1].

- The case of almost minimizers in the finite dimensional setting. Here $T \in$ $\mathscr{R}_{m}\left(\ell_{2}^{n} ; \mathbb{Z}\right)$ is almost mass minimizing. The regularity has been established by E. Bombieri [8] following the scheme of proof set forth by F.J. Almgren [2] in the framework of sets rather than chains.

- The case of minimizers in the infinite dimensional setting. Here $T \in$ $\mathscr{R}_{m}\left(\ell_{2} ; \mathbb{Z}\right)$ is mass minimizing. L. Ambrosio, C. De Lellis and T. Schmidt [4] have established the result in the framework of "currents in metric spaces" $[6]$.

A $C^{1}$ version of the regularity theorem holds when the bound $C r^{\alpha}$ quantifying the almost minimizing property is replaced by a coarser bound that decays fast enough, see the remark after Theorem 5.13. In Section 6 we show that the discreteness of $G$ is necessary and give an example of a mass minimizing chain, with coefficients in a totally disconnected compact normed group, and without any regular point, Example 6.1. Proposition 6.2 shows that any chain induced by a $C^{1, \alpha}$ submanifold of $X$ is almost mass minimizing, as we claimed above, and more generally the sum of finitely many $C^{1, \alpha}$ submanifolds that "nicely" intersect in a common set $\Sigma$ is 
almost mass minimizing as well. Taking for $\Sigma$ a Cantor set of positive $\mathscr{H}^{m}$ measure we show that the set of regular points need not be co-null in general, Example 6.3.

One serious difficulty with working in infinite dimension is the lack of certain compactness results that are specific to a finite dimensional ambient space. Specifically, the essential Excess Decay Lemma is established in [2] and [8] by contradiction, a delicate argument based on compactness. Such reasoning seems to be bound to fail in $\ell_{2}$. We now turn to briefly describing the scheme of proof of Theorem 1.1.

Let $T \in \mathscr{R}_{m}(X ; G)$. We let $\|T\|$ denote the finite Borel measure in $X$ associated with $T$, i.e. $\|T\|(B)=\int_{B}\left\|\mathbf{g}_{T}\right\| d \mathscr{H}^{m}\left\llcorner M_{T}\right.$ where $M_{T}$ and $\mathbf{g}_{T}$ are respectively the $m$ dimensional rectifiable set and the $G$ orientation of $T$. Let $a \in X, r>0$ and $W \in \mathbf{G}(X, m)$. Assume for definiteness $\Theta^{m}(\|T\|, a)=1$. We consider the following two quantities.

$$
\mathbf{e x c}^{m}(\|T\|, a, r)=\frac{\|T\|(\mathbf{B}(a, r))}{\boldsymbol{\alpha}(m) r^{m}}-1
$$

and

$$
\beta_{2}^{2}(\|T\|, a, r, W)=\frac{1}{r^{m+2}} \int_{\mathbf{B}(a, r)} \operatorname{dist}^{2}(y-a, W) d\|T\|(y) .
$$

The first one we call the spherical excess. To get a sense of what these quantify, assume $\operatorname{spt}(T) \cap \mathbf{B}(a, r)$ coincides with $\left(a+\Gamma_{f}\right) \cap \mathbf{B}(a, r)$ where $\Gamma_{f}$ is the graph of some $f: W \rightarrow W^{\perp}$ with small Lipschitz constant so that $\left(a+\Gamma_{f}\right) \cap \mathbf{B}(a, r)$ is contained in a slab around $a+W$ whose height is small with respect to $r$. Upon considering cylindrical versions of $\mathbf{e x c}^{m}$ and $\beta_{2}$, and calculating the Taylor expansion of the Hilbertian Jacobian of $f$, one can check that

$$
\operatorname{exc}^{m}(\|T\|, a, r) \cong \frac{1}{\boldsymbol{\alpha}(m) r^{m}} \int_{W \cap \mathbf{B}(0, r)}\|D f\|_{\mathrm{HS}}^{2} d \mathscr{H}^{m}
$$

(where $\|\cdot\|_{\text {HS }}$ stands for the Hilbert-Schmidt norm), and

$$
\beta_{2}^{2}(\|T\|, a, r, W) \cong \frac{1}{r^{m+2}} \int_{W \cap \mathbf{B}(0, r)}|f|^{2} d \mathscr{H}^{m} .
$$

It is classical to check that if $T$ is mass minimizing then $\mathbf{e x c}^{m}(\|T\|, a, r)$ is a nondecreasing function of $0<r<\operatorname{dist}(a, \operatorname{spt}(\partial T))$, for every $a \notin \operatorname{spt}(\partial T)$. In case $T$ is almost mass minimizing, the spherical excess is almost nondecreasing in the sense that $\exp \left[C r^{\alpha}\right] \frac{\|T\|(\mathbf{B}(a, r))}{\boldsymbol{\alpha}(m) r^{m}}-1$ is nondecreasing. This is a useful fact used throughout the paper. The three steps of the proof are now the following.

(H) Identifying a set of hypotheses called "closeness to flat" that guarantee that $T$ is sufficiently close to flat in some ball $\mathbf{B}(a, r)$, both in the sense that $\operatorname{spt}(T) \cap \mathbf{B}(a, r)$ is close in Hausdorff distance to $(a+W) \cap \mathbf{B}(a, r)$ for some $W \in \mathbf{G}(X, m)$, and in the sense that $\|T\|(\mathbf{B}(a, r))$ is close to the measure $\boldsymbol{\alpha}(m) r^{m}$ of the $m$ dimensional disk $(a+W) \cap \mathbf{B}(a, r)$ weighted by the "local coefficient" of $T$ near $a$. The existence of such $W$ by no means implies that it is the "tangent space" to $\operatorname{spt}(T)$ at $a$ and the remaining part of the analysis consists in estimating how much these $W$ may vary as $r$ tends to 0 . Another feature of the "closeness to flat" assumption is that it should be verified by an open dense set of points in the support. This is where the assumption that $G$ be discrete comes into play.

(1) Showing that, under $(\mathrm{H})$, for $a \in \operatorname{spt}(T)$ the spherical excess decays fast enough, specifically $\operatorname{exc}^{m}(\|T\|, a, r) \leq C r^{\gamma}$ for some $\gamma>0$. 
(2) Showing that, under $(\mathrm{H})$, there exists $W_{a, r} \in \mathbf{G}(X, m)$ (not necessarily the same as in the assumption $(\mathrm{H}))$ such that $\beta_{2}^{2}\left(\|T\|, a, r, W_{a, r}\right) \leq$ $C \operatorname{exc}^{m}(\|T\|, a, r)^{\beta}$. It will then ensue from (2) that $W_{a}=\lim _{r \rightarrow 0} W_{a, r}$ exists, and that $W_{a}$ and $W_{b}$ are close to one another according to how $a$ and $b$ are close. This is enough to finish off the proof.

Let us now briefly comment on steps (1) and (2). To keep the notations short we assume $a=0$ and we abbreviate $\phi_{T}(r)=\|T\|(\mathbf{B}(0, r)), \mathbf{e}_{T}(r)=\mathbf{e x c}^{m}(\|T\|, 0, r)$, and for convenience we introduce the non normalized version of the spherical excess, namely $e_{T}(r)=\operatorname{exc}^{m}(\|T\|, 0, r)=\boldsymbol{\alpha}(m) r^{m} \operatorname{exc}^{m}(\|T\|, 0, r)=\phi_{T}(r)-\boldsymbol{\alpha}(m) r^{m}$. We ought to determine whether $\mathbf{e}_{T}(r) \leq C r^{\gamma}$, which is equivalent to $e_{T}(r) \leq C r^{m(1+\epsilon)}$ where we have abbreviated $\epsilon=\gamma m^{-1}$. In case $T$ is mass minimizing $\mathbf{e}_{T}(r)$ is nondecreasing and nonnegative. In case $T$ is merely almost mass minimizing we would have to introduce a multiplicative factor $\exp \left[\mathrm{Cr}^{\alpha}\right]$ in the computations, which we omit in these introductory remarks. Thus assuming that $e_{T}(r) \geq 0$, the growth $e_{T}(r) \leq C r^{m(1+\epsilon)}$ will follow from the differential inequality

$$
m(1+\epsilon) \frac{d}{d r} \log r \leq \frac{d}{d r} \log e_{T}(r),
$$

which is thus equivalent to

$$
(1+\epsilon) e_{T}(r) \leq \frac{r}{m} e_{T}^{\prime}(r) .
$$

In estimating $r m^{-1} e_{T}^{\prime}(r)=r m^{-1}\left(\phi_{T}^{\prime}(r)-m \boldsymbol{\alpha}(m) r^{m-1}\right)=r m^{-1} \phi_{T}^{\prime}(r)-\boldsymbol{\alpha}(m) r^{m}$ we observe that

$$
\frac{r}{m} \phi_{T}^{\prime}(r) \geq \frac{r}{m} \mathbf{M}(\langle T,|\cdot|, r\rangle)=\mathbf{M}(\llbracket 0 \rrbracket \nVdash\langle T,|\cdot|, r\rangle)=: \phi_{C_{r}}(r)
$$

is the measure in $\mathbf{B}(0, r)$ of the cone $C_{r}$ with vertex 0 and with base the slice

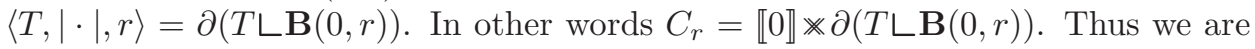
asking whether

$$
(1+\epsilon) e_{T}(r) \leq \phi_{C_{r}}(r)-\boldsymbol{\alpha}(m) r^{m}=e_{C_{r}}(r) .
$$

The inequality $e_{T}(r) \leq \theta e_{C_{r}}(r)$, for some $0<\theta<1$, is the quantitative improvement on the monotonicity inequality that we call the "Reifenberg epiperimetric inequality". In order to evoke its proof let us assume that $r=1$ and that $\operatorname{spt}(T) \cap Z_{W}(0,1)=\Gamma_{f_{W}} \cap Z_{W}(0,1)$ for some $W \in \mathbf{G}(X, m)$ and some $f_{W}: W \rightarrow W^{\perp}$, where $Z_{W}(0,1)$ denotes the cylinder $\pi_{W}^{-1}(W \cap \mathbf{B}(0,1))$. We let $h_{W}$ be the homogeneous extension of degree 1 , to $W \cap \mathbf{B}(0,1)$, of $\left.f_{W}\right|_{\operatorname{Bdry}(W \cap \mathbf{B}(0,1))}$. The question is then (up to error terms due to the replacement of a ball by a cylinder, and which are small with respect to $\left.e_{T}(1)\right)$ whether the following holds:

$$
\int_{W \cap \mathbf{B}(0,1)}\left\|D f_{W}\right\|_{\mathrm{HS}}^{2} d \mathscr{H}^{m} \leq \theta \int_{W \cap \mathbf{B}(0,1)}\left\|D h_{W}\right\|_{\mathrm{HS}}^{2} d \mathscr{H}^{m} .
$$

To see that this cannot be true in general it suffices to observe that if $h_{W}$ is linear and nonzero then it is harmonic and hence a solution of the Dirichlet problem: It minimizes its Dirichlet energy among those maps having the same boundary values and thus $f_{W}$ cannot have strictly less Dirichlet energy. This drawback can be overcome if we can make sure $h_{W}$ is far enough from being linear, which amounts to choosing $W$ initially so as to cancel the linear part of $h_{W}$. This should mean 
that, if possible, $h_{W}$ is orthogonal to linear functions in the Lebesgue space $\mathbf{L}_{2}(W \cap$ $\left.\mathbf{B}(0,1), \mathscr{H}^{m}\right)$. In other words we seek for a $W \in \mathbf{G}(X, m)$ such that

$$
\int_{W \cap \mathbf{B}(0,1)}\left\langle h_{W}(x), v\right\rangle\langle x, u\rangle d \mathscr{H}^{m}(x)=0
$$

for every unit vectors $u \in W$ and $v \in W^{\perp}$. E.R. Reifenberg solves this problem by minimizing a functional over the Grassmannian $\mathbf{G}\left(\ell_{2}^{n}, m\right)$. In the infinite dimensional setting the Grassmannian lacks the compactness required to make this work and instead we argue as follows. Abbreviating $C=C_{1}=\llbracket 0 \rrbracket \circledast \partial(T\llcorner\mathbf{B}(0,1))$, we consider the quadratic form

$$
Q_{C}(x)=\int_{\mathbf{B}(0,1)}\langle x, y\rangle^{2} d\|C\|(y)=\left\langle x, L_{C}(x)\right\rangle
$$

where

$$
L_{C}(x)=\int_{\mathbf{B}(0,1)} y\langle x, y\rangle d\|C\|(y) .
$$

We notice that $L_{C}$ is a positive self-adjoint compact operator on $X$ and therefore $X$ admits an orthonormal basis of eigenvectors $e_{1}, e_{2}, \ldots$ of $L_{C}$, according to the spectral theorem. We order these as usual so that $\lambda_{1} \geq \lambda_{2} \geq \ldots \geq 0$ for the corresponding eigenvalues. In fact, since we assume $T$ (and hence $C$ ) to be sufficiently close to flat in $\mathbf{B}(0,1), Q_{C}$ is close to a multiple of $\left|\pi_{W}(\cdot)\right|^{2}$. Thus if $\hat{W} \in \mathbf{G}(X, m)$ is generated by $e_{1}, \ldots, e_{m}$ then $\hat{W}$ is close to $W$ (appearing in $(\mathrm{H}))$. Moreover $L_{C}\left(e_{k}\right)=\lambda_{k} e_{k}$ for every $k$, and therefore $\left\langle e_{j}, L_{C}\left(e_{k}\right)\right\rangle=0$ for all $j=1, \ldots, m$ and all $k=m+1, m+2, \ldots$, which is (1.2) up to some small error terms depending on the initial closeness to flat. With $W$ replaced by $\hat{W}$ it will be possible to find $f_{\hat{W}}$ with boundary values same as $h_{\hat{W}}$ and achieving the improvement of (1.1). Of course the graph of $f_{\hat{W}}$ may not meet at all the support of $T$ in $\mathbf{B}(0,1)$, yet

$$
\int_{\hat{W} \cap \mathbf{B}(0,1)}\left\|D f_{\hat{W}}\right\|_{\mathrm{HS}}^{2} d \mathscr{H}^{m} \gtrsim e_{T}(1)
$$

according to the almost mass minimizing property of $T$. We can now infer that $\mathbf{e}_{T}(1) \leq \theta \mathbf{e}_{C}(1)$. Our proof follows very closely the arguments of E.R. Reifenberg in [20]. The essential differences are the averaging of the different layers to account for the elements of the normed group $G$ and the use of the quadratic form $Q_{C}$ as explained above.

At this point it is perhaps worth saying a word on how to obtain $f_{W}$ in (1.1) provided (1.2) holds. One way would be to find the optimal $f_{W}$ for the problem, i.e. the mapping $f_{W}$ each of whose coordinates is harmonic. This is indeed how E.R. Reifenberg proceeded. Nevertheless the computations that establish (1.1), involving identities for spherical harmonics, work as well for any homogeneous extension of $h_{W}$, of degree $1+t>1$ (with $\theta$ depending on $t$ ). In other words, contrary to popular belief, there exist proofs of partial regularity for (almost) mass minimizing chains that do not involve at any stage the use of harmonic functions. Sure enough, after we realized this, we found out that C.B. Morrey had also reported this fact in [17, Lemma 10.6.13].

Finally we turn to briefly discussing step (2) of our proof, i.e. the sort of Poincaré inequality with an exponent, $\beta_{2}^{2}(\|T\|, a, r, W) \leq C \operatorname{exc}^{m}(T, a, r)^{\beta}$ whenever $(\mathrm{H})$ holds. In fact, according to the Ahlfors regularity of $\operatorname{spt}(T)$ (a consequence of almost monotonicity), this is equivalent to $\beta_{\infty}(\|T\|, a, r, W) \leq \operatorname{exc}^{m}(\|T\|, a, r)^{\beta}$ (for some 
different $0<\beta<1$ ). Readers familiar with regularity proofs will recognize here a height bound, usually obtained as a corollary of graphical Lipschitz approximation of the support, and that would definitely be one way to go about it for instance when $G=\mathbb{Z}$. Here, as we used a quadratic form $Q$ associated to $T$ already, we choose to be consistent and keep using it. This sort of moments computations has been set forth in [18], and a quantitative version was described in [11]. We review the idea behind it and point out the differences with [11].

Assume for simplicity $a=0 \in \operatorname{spt}(T)$. We notice that according to Cavalieri's principle, information about the growth of $\|T\|(\mathbf{B}(0, r))$ is equivalent to information about the growth of

$$
\hat{V}(\|T\|, x, r)=\int_{\mathbf{B}(x, r)}\left(r^{2}-|x-y|^{2}\right)^{2} d\|T\|(y) .
$$

As its variable $x$ appears in the moving domain of integration, this function $\hat{V}$ does not immediately occur as being differentiable in $x$. We therefore introduce a slight variation of $\hat{V}$, the polynomial function

$$
V(\|T\|, x, r)=\int_{\mathbf{B}(0, r)}\left(r^{2}-|x-y|^{2}\right)^{2} d\|T\|(y)=\sum_{k=0}^{4} P_{k}(\|T\|, x, r)
$$

which is expanded in a sum of $P_{k}$ 's, polynomials in $x$ homogeneous of degree $k$. A simple computation yields

$$
|V(\|T\|, x, r)-\hat{V}(\|T\|, x, r)| \leq C\left(r^{m+1}|x|^{3}+r^{m+2+\beta}|x|^{2}\right) .
$$

From this we infer that if we divide $V$ and $\hat{V}$ by $r^{m+2}$ the corresponding functions will be close up to $O\left(|x|^{2}\right)$ when $|x| \leq r$, and up to $o\left(|x|^{2}\right)$ when $|x|=o(1) r$, so that information about the variations of $\|T\|(\mathbf{B}(0, r))$, and in turn about the variations of $\hat{V}$, translates to information about the polynomials $P_{k}$. We then use the fact that $P_{2}$ normalizes to a dimensionless quantity when divided by $r^{m+2}$, and contains a term akin to $Q$ defined above. Specifically,

$$
P_{2}(\|T\|, x, r)=4 Q_{T}(x, r)-2|x|^{2} \int_{\mathbf{B}(0, r)}\left(r^{2}-|y|^{2}\right) d\|T\|(y)
$$

where, as above,

$$
Q_{T}(x, r)=\int_{\mathbf{B}(0, r)}\langle x, y\rangle^{2} d\|T\|(y),
$$

and if

$$
\mathbf{P}_{2}=\frac{m+2}{\boldsymbol{\alpha}(m) r^{m+2}} P_{2} \text { and } \mathbf{Q}_{T}=\frac{m+2}{\boldsymbol{\alpha}(m) r^{m+2}} Q_{T}
$$

then, for instance,

$$
\left|\operatorname{tr} \mathbf{Q}_{T}(\cdot, r)-m\right| \leq C r^{\gamma^{\prime}}
$$

under $(\mathrm{H})$, where $\gamma^{\prime}$ is a function of $\gamma$ appearing in step (1). Assuming for now that the first moment $P_{1}$, which is a version of a center of mass of $\|T\|$ in $\mathbf{B}(0, r)$ that initially normalizes as $r^{m+1}$, can be made as small as $C r^{\gamma^{\prime}}$ when divided by $r^{m+2}$, we end up showing that

$$
\left.\left.\left|\mathbf{Q}_{T}(x, r)-\right| x\right|^{2}\left|\leq C r^{\gamma^{\prime}}\right| x\right|^{2}
$$


whenever $x \in \operatorname{spt}(T), \Theta^{m}(\|T\|, x)=1$ and $|x|=r^{1+\gamma^{\prime}}$. If we manage to find orthogonal vectors $x_{1}, \ldots, x_{m} \in \operatorname{spt}(T)$ such that $\left|x_{i}\right|=r^{1+\gamma^{\prime}}$ and $\Theta^{m}\left(\|T\|, x_{i}\right)=1$, then we infer from the inequality above, from the definition of $P_{2}$ and from (1.3) that

$$
\frac{1}{r^{m+2}} \int_{\mathbf{B}(0, r)} \operatorname{dist}^{2}(y, W) d\|T\|(y) \leq C r^{\gamma^{\prime}}
$$

and we will be done. In [11] the orthogonal frame $x_{1}, \ldots, x_{m}$ in $\operatorname{spt}(T)$ was found because $\operatorname{spt}(T)$ was shown to be Reifenberg flat under $(\mathrm{H})$, and therefore a topological $m$ disk according to [20]. The Reifenberg flatness however followed by a compactness argument not available in infinite dimension. Instead we take advantage of the fact that $T$ is rectifiable and hence for $\|T\|$ almost every $a$ we can assume we look at a scale $r(a)$ small enough to start with that $\operatorname{spt}(T) \cap \mathbf{B}(a, r)$ is close to its tangent space $W_{a}$ at $a$ (notice a tangent space exists because $\ell_{2}$ has the Radon-Nikodým property, and closeness occurs in Hausdorff distance thanks to Ahlfors regularity). At that small scale $r(a)$ (and all smaller scales) we can find $x_{1}, \ldots, x_{m}$ with the required properties. We then show in 5.8, using a backward bootstrap argument that the same holds at larger scales $r$ provided the density ratio at scale $r$ is not much larger than 1 . This can be done at neighboring points up the same largest scale $r_{0}$ thanks to almost monotonicity, see 5.7. To summarize, we establish that (1.4) now holds at scales uniformly small $0<r \leq r_{0}$ and that $\operatorname{spt}(T) \cap \mathbf{B}(a, r)$ is in fact Reifenberg flat, 5.9. It then classically follows from (1.4) that $\operatorname{spt}(T) \cap \mathbf{B}(a, r)$ is $C^{1, \gamma^{\prime \prime}}$.

\section{Preliminary Results for Chains in a Hilbert space}

Throughout these notes $(X,\langle\cdot, \cdot\rangle)$ is a separable Hilbert space with $\operatorname{dim}(X)>m$, and $T \in \mathscr{R}_{m}(X ; G)$ is a chain with coefficients in a complete normed Abelian group $(G,\|\cdot\|)$. A norm on an Abelian group is a function $\|\cdot\|: G \rightarrow[0, \infty)$ such that for all $g, h \in G$,

(1) $\|-g\|=\|g\|$,

(2) $\|g+h\| \leq\|g\|+\|h\|$,

(3) $\|g\|=0$ if and only if $g=0_{G}$.

The vector space norm on $X$ is denoted by $|\cdot|$ to distinguish it from the one on $G$. Since the support of $T$ is a separable subset of $X$, the statements we make remain true for any Hilbert ambient space because we can always restrict to a separable complete subspace thereof. The Grassmannian of $m$-dimensional subspaces of $X$ is denoted by $\mathbf{G}(X, m)$. Given an $m$-plane $W \in \mathbf{G}(X, m)$ we denote by $\pi_{W}$ the orthogonal projection of $X$ onto $W$. We often use these projections as push-forwards for chains. In order to speak about chains on a plane $W \in \mathbf{G}(X, m)$ it is necessary that we choose an orientation on $W$. This orientation is mostly not so important since we are interested in the mass of the chains which is independent of this choice. In case we work with a collection of planes that are close to each other, we implicitly assume that these orientations are compatible in the sense that the orthogonal projection from one to the other is orientation preserving.

With $\mathbf{B}(x, r)$ and $\mathbf{U}(x, r)$ we denote the closed, respectively open, ball of radius $r \geq 0$ around $x \in X$. Sometimes we may also use $\mathbf{B}(r):=\mathbf{B}(0, r), \mathbf{U}(r):=\mathbf{U}(0, r)$, $\mathbf{B}:=\mathbf{B}(0,1)$ and $\mathbf{U}:=\mathbf{U}(0,1)$. Similarly, for $A \subset X$ we denote by $\mathbf{B}(A, r)$ and $\mathbf{U}(A, r)$ the closed, respectively open, tubular neighborhood of radius $r$ around $A$. 
For intersections we use $\mathbf{B}_{A}(x, r):=\mathbf{B}(x, r) \cap A$ and $\mathbf{U}_{A}(x, r):=\mathbf{U}(x, r) \cap A$. The cylinder around $x \in X$ of radius $r$ above an $m$-dimensional plane $W \in \mathbf{G}(X, m)$ is given by $Z_{W}(x, r):=\pi_{W}{ }^{-1}\left(\mathbf{B}_{W}\left(\pi_{W}(x), r\right)\right)$, or in special cases $Z_{W}(r):=Z_{W}(0, r)$ and $Z_{W}:=Z_{W}(0,1) \cdot \boldsymbol{\alpha}(m)$ denotes the volume of the unit ball in $\mathbb{R}^{m}$. For two nonempty subsets $A, A^{\prime} \subset X$ the Hausdorff distance is

$$
\begin{aligned}
d_{\mathrm{H}}\left(A, A^{\prime}\right) & :=\inf \left\{r>0: A \subset \mathbf{B}\left(A^{\prime}, r\right), A^{\prime} \subset \mathbf{B}(A, r)\right\} \\
& =\max \left\{\sup _{x \in A} \operatorname{dist}\left(x, A^{\prime}\right), \sup _{x^{\prime} \in A^{\prime}} \operatorname{dist}\left(x^{\prime}, A\right)\right\} .
\end{aligned}
$$

In the rest of this section we describe some of the basic tools we need in the process.

2.1. Grassmannian and Hausdorff distance. The Grassmannian $\mathbf{G}(X, m)$ can be equipped with a complete metric. Two natural definitions of a metric are shown to be equivalent in the following lemma. Here $\|\cdot\|$ denotes the operator norm.

2.1. Lemma. Let $m \geq 1$ be an integer and $V_{1}, V_{2} \in \mathbf{G}(X, m)$. The following hold:

(1) $\left\|\pi_{V_{1}}-\pi_{V_{2}}\right\|=d_{H}\left(V_{1} \cap \boldsymbol{B}(0,1), V_{2} \cap \boldsymbol{B}(0,1)\right)$;

(2) $\| \pi_{V_{1}}-\pi_{V_{2}}||=\max \left\{\left|v-\pi_{V_{2}}(v)\right|: v \in V_{1} \cap \boldsymbol{B}(0,1)\right\}$;

(3) $\left\|\pi_{V_{2}^{\perp}} \circ \pi_{V_{1}}\right\| \leq\left\|\pi_{V_{1}}-\pi_{V_{2}}\right\|$.

Proof. We start with the trivial observation that $\operatorname{dist}\left(v, V_{i} \cap \mathbf{B}(0,1)\right)=\operatorname{dist}\left(v, V_{i}\right)$ whenever $v \in \mathbf{B}(0,1), i=1,2$, which will be used repeatedly without further notice. Next we observe that

$$
\max \left\{\operatorname{dist}\left(v, V_{2}\right): v \in V_{1} \cap \mathbf{B}(0,1)\right\} \leq\left\|\pi_{V_{1}}-\pi_{V_{2}}\right\| .
$$

Indeed suppose $v \in V_{1} \cap \mathbf{B}(0,1)$, then

$$
\operatorname{dist}\left(v, V_{2}\right)=\left|\pi_{V_{2}^{\perp}}(v)\right|=\left|\operatorname{id}_{X}(v)-\pi_{V_{2}}(v)\right|=\left|\pi_{V_{1}}(v)-\pi_{V_{2}}(v)\right| \leq\left\|\pi_{V_{1}}-\pi_{V_{2}}\right\|
$$

which establishes (2.1). Swapping $V_{1}$ and $V_{2}$ in (2.1) we obtain

$$
d_{\mathrm{H}}\left(V_{1} \cap \mathbf{B}(0,1), V_{2} \cap \mathbf{B}(0,1)\right) \leq\left\|\pi_{V_{1}}-\pi_{V_{2}}\right\| .
$$

In order to prove conclusion (1) it remains to prove the reverse inequality:

$$
\left\|\pi_{V_{1}}-\pi_{V_{2}}\right\| \leq \max \left\{\max _{v \in V_{1} \cap \mathbf{B}(0,1)} \operatorname{dist}\left(v, V_{2}\right), \max _{v \in V_{2} \cap \mathbf{B}(0,1)} \operatorname{dist}\left(v, V_{1}\right)\right\} .
$$

We prove inequality (2.3) first in case $m=1$ and $\operatorname{dim}(X)=2$. We leave to the reader the simple computation showing that, in this special case, the map $X \cap \mathbf{S}(0,1) \rightarrow \mathbb{R}: x \mapsto\left|\pi_{V_{1}}(x)-\pi_{V_{2}}(x)\right|$ is constant. Therefore

$$
\| \pi_{V_{1}}-\pi_{V_{2}}||=\left|\pi_{V_{1}}(v)-\pi_{V_{2}}(v)\right|=\left|\pi_{V_{2}^{\perp}}(v)\right|=\operatorname{dist}\left(v, V_{2}\right),
$$

for every $v \in V_{1} \cap \mathbf{S}(0,1)$.

Next we assume $m \geq 2$ and $\operatorname{dim}(X)<\infty$. We choose $v^{*}$ which maximizes $\mathbf{S}(0,1) \rightarrow \mathbb{R}: v \mapsto\left|\pi_{V_{1}}(v)-\pi_{V_{2}}(v)\right|^{2}$, so that $\left\|\pi_{V_{1}}-\pi_{V_{2}}\right\|=\left|\pi_{V_{1}}\left(v^{*}\right)-\pi_{V_{2}}\left(v^{*}\right)\right|$. According to the Lagrange Multiplier Theorem there exists $\lambda \in \mathbb{R}$ such that $\lambda v^{*}=$ $\pi_{V_{1}}\left(v^{*}\right)-\pi_{V_{2}}\left(v^{*}\right)$. If $\lambda=0$ then $\left\|\pi_{V_{1}}-\pi_{V_{2}}\right\|=0$ and (2.3) clearly holds. We henceforth assume $\lambda \neq 0$. If $\pi_{V_{1}}\left(v^{*}\right)=0$ then $v^{*} \in V_{2}$, consequently $\left\|\pi_{V_{1}}-\pi_{V_{2}}\right\|=$ $\left|\pi_{V_{2}}\left(v^{*}\right)\right|=\left|v^{*}\right|=1=\left|v^{*}-\pi_{V_{1}}\left(v^{*}\right)\right| \leq \max \left\{\operatorname{dist}\left(v, V_{1}\right): v \in V_{2} \cap \mathbf{B}(0,1)\right\}$, thus (2.3) holds. Similarly if $\pi_{V_{2}}\left(v^{*}\right)=0$ then $\left\|\pi_{V_{1}}-\pi_{V_{2}}\right\| \leq \max \left\{\operatorname{dist}\left(v, V_{2}\right): v \in\right.$ $\left.V_{1} \cap \mathbf{B}(0,1)\right\}$, and $(2.3)$ holds as well. Assuming now that $\pi_{V_{1}}\left(v^{*}\right) \neq 0 \neq \pi_{V_{2}}\left(v^{*}\right)$ we define $V:=\operatorname{span}\left\{\pi_{V_{1}}\left(v^{*}\right), \pi_{V_{2}}\left(v^{*}\right)\right\}$, so that $v \in V$. We note $\operatorname{dim} V=2$ (assuming if possible that $\operatorname{dim} V=1$, it would ensue $\pi_{V_{1}}\left(v^{*}\right)=t \cdot \pi_{V_{2}}\left(v^{*}\right)$ for some $t \neq 0$, and 
THIERRY DE PAUW AND ROGER ZÜST

in turn $v^{*}=\lambda^{-1}(t-1) \pi_{V_{2}}\left(v^{*}\right) \in V_{2}$, thus also $v^{*}=\pi_{V_{2}}\left(v^{*}\right)=t^{-1} \pi_{V_{1}}\left(v^{*}\right) \in V_{1}$, whence $\lambda v^{*}=v^{*}-v^{*}=0$, a contradiction), as well as $\operatorname{dim} V \cap V_{1}=1=\operatorname{dim} V \cap V_{2}$. In other words we may apply the previous 2 dimensional case applies to $V, V \cap V_{1}$ and $V \cap V_{2}$. To this end we notice that $\pi_{v_{i}}(v)=\pi_{V \cap V_{i}}(v)$ whenever $v \in V$ (it suffices to observe that $\pi_{V_{i}}(v) \in V$ for $v=\pi_{V_{j}}\left(v^{*}\right), j=1,2$, which is obvious if $j=i$ whereas if $j=2, i=1$ then $\pi_{V_{2}}\left(\pi_{V_{1}}\left(v^{*}\right)\right)=(\lambda+1) \pi_{V_{2}}\left(v^{*}\right) \in V$, and the other case is similar). Thus,

$$
\begin{aligned}
\left\|\pi_{V_{1}}-\pi_{V_{2}}\right\| & =\left|\pi_{V_{1}}\left(v^{*}\right)-\pi_{V_{2}}\left(v^{*}\right)\right| \\
& =\left|\pi_{V \cap V_{1}}\left(v^{*}\right)-\pi_{V \cap V_{2}}\left(v^{*}\right)\right| \\
& \leq \| \pi_{V \cap V_{1}}\left\lceil V-\pi_{V \cap V_{2}} \uparrow V \|\right. \\
& \leq \max \left\{\max _{v \in V \cap V_{1} \cap \mathbf{B}(0,1)} \operatorname{dist}\left(v, V \cap V_{2}\right), \max _{v \in V \cap V_{2} \cap \mathbf{B}(0,1)} \operatorname{dist}\left(v, V \cap V_{1}\right)\right\} \\
& =\max \left\{\max _{v \in V \cap V_{1} \cap \mathbf{B}(0,1)} \operatorname{dist}\left(v, V_{2}\right), \max _{v \in V \cap V_{2} \cap \mathbf{B}(0,1)} \operatorname{dist}\left(v, V_{1}\right)\right\} \\
& \leq \max \left\{\max _{v \in V_{1} \cap \mathbf{B}(0,1)} \operatorname{dist}\left(v, V_{2}\right), \max _{v \in V_{2} \cap \mathbf{B}(0,1)} \operatorname{dist}\left(v, V_{1}\right)\right\} \\
& \leq\left\|\pi_{V_{1}}-\pi_{V_{2}}\right\| .
\end{aligned}
$$

This completes the proof of $(2.3)$ in case $\operatorname{dim}(X)<\infty$ and we leave it to the reader to check it also holds when $\operatorname{dim}(X)=\infty$. Conclusion (1) now readily follows, whereas conclusion (2) holds because it holds in case $\operatorname{dim} X=2$ and all inequalities above are equalities.

In order to prove (3) we simply notice that

$$
\begin{aligned}
\left\|\pi_{V_{2}^{\perp}} \circ \pi_{V_{1}}\right\| & =\left\|\left(\mathrm{id}_{X}-\pi_{V_{2}}\right) \circ \pi_{V_{1}}\right\| \\
& \left.=\|\left(\operatorname{id}_{X}-\pi_{V_{2}}\right) \circ\left(\pi_{V_{1}}-\pi_{V_{2}}\right)+\left(\operatorname{id}_{X}-\pi_{V_{2}}\right) \circ \pi_{V_{2}}\right) \| \\
& \leq\left\|\pi_{V_{2}^{\perp}}\right\|\left\|\pi_{V_{1}}-\pi_{V_{2}}\right\| .
\end{aligned}
$$

We will need some estimates on the closeness of different planes that approximate some set $S \subset X$. Such estimates are established in the results below.

2.2. Definition. Let $m>0$ be an integer, $S \subset X, x \in S, r>0$ and $\epsilon>0$. We say that $S$ is $\epsilon$ flat at $(x, r)$ if there exists $V \in \mathbf{G}(X, m)$ such that

$$
d_{\mathrm{H}}(S \cap \mathbf{B}(x, r),(x+V) \cap \mathbf{B}(x, r)) \leq \epsilon r .
$$

We also define

$$
\mathbf{G}(S, x, r, \epsilon):=\left\{V \in \mathbf{G}(X, m): d_{\mathrm{H}}(S \cap \mathbf{B}(x, r),(x+V) \cap \mathbf{B}(x, r)) \leq \epsilon r\right\} .
$$

The dimension $m$ does not appear either in the notation or in the terminology since it will always be clear from the context. We leave it to the reader to check the trivial and useful fact that $\mathbf{G}(X, m) \subset \mathbf{G}(S, x, r, \epsilon)$ whenever $\epsilon \geq 1$.

\subsection{Lemma. Assume that}

(1) $S \subset X, x \in S, R>0, \epsilon>0$ and $0<\lambda \leq 1$;

(2) $V \in \mathbf{G}(S, x, R, \epsilon)$.

Then $V \in \mathbf{G}\left(S, x, \lambda R, 2 \epsilon \lambda^{-1}\right)$. 
Proof. If $\lambda \leq \epsilon$ then there is nothing to prove, according to the remark right before the Lemma. Thus we assume from now on that $\epsilon<\lambda$. Let $\zeta \in S \cap \mathbf{B}(x, \lambda R) \subset$ $S \cap \mathbf{B}(x, R)$. Define $\xi:=x+\pi_{V}(\zeta-x)$. Since $|\xi-x|=\left|\pi_{V}(\zeta-x)\right| \leq|\zeta-x| \leq \lambda R$ one has $\xi \in(x+V) \cap \mathbf{B}(x, \lambda R)$. Furthermore,

$$
|\xi-\zeta|=\operatorname{dist}(\zeta, x+V) \leq \operatorname{dist}(\zeta,(x+V) \cap \mathbf{B}(x, R)) \leq \epsilon R
$$

by assumption (2). It follows that

$$
S \cap \mathbf{B}(x, \lambda R) \subset \mathbf{B}\left[(x+V) \cap \mathbf{B}(x, \lambda R),\left(\epsilon \lambda^{-1}\right) \lambda R\right] .
$$

Let now $\xi \in(x+V) \cap \mathbf{B}(x, \lambda R)$ and $\epsilon<\hat{\epsilon}<\lambda$. Define $\xi^{\prime}=x+\lambda^{-1}(\lambda-\hat{\epsilon})(\xi-x)$ and observe that $\left|\xi^{\prime}-x\right|=\lambda^{-1}(\lambda-\hat{\epsilon})|\xi-x| \leq(\lambda-\hat{\epsilon}) R \leq R$. In particular $\xi^{\prime} \in(x+V) \cap \mathbf{B}(x, R)$ and according to assumption (2) there exists $\zeta \in S \cap \mathbf{B}(x, R)$ such that $\left|\xi^{\prime}-\zeta\right| \leq \hat{\epsilon} R$. Note that

$$
|\zeta-x| \leq\left|\zeta-\xi^{\prime}\right|+\left|\xi^{\prime}-x\right| \leq \hat{\epsilon} R+(\lambda-\hat{\epsilon}) R=\lambda R,
$$

whence in fact $\zeta \in S \cap \mathbf{B}(x, \lambda R)$, and also

$$
\begin{aligned}
|\zeta-\xi| \leq\left|\zeta-\xi^{\prime}\right|+\left|\xi^{\prime}-\xi\right| \leq \hat{\epsilon} R+\left|1-\lambda^{-1}(\lambda-\hat{\epsilon})\right| \cdot|\xi-x| & \\
& \leq \hat{\epsilon} R+\hat{\epsilon} \lambda^{-1} \lambda R=2 \hat{\epsilon} R .
\end{aligned}
$$

Therefore

$$
(x+V) \cap \mathbf{B}(x, \lambda R) \subset \mathbf{B}\left[S \cap \mathbf{B}(x, \lambda R),\left(2 \hat{\epsilon} \lambda^{-1}\right) \lambda R\right]
$$

and the conclusion follows from the arbitrariness of $\hat{\epsilon}>\epsilon$.

2.4. Corollary (Same center, different scales). Assume that

(1) $S \subset X, x \in S, \epsilon>0$ and $0<r<R$;

(2) $V_{r} \in \mathbf{G}(S, x, r, \epsilon)$ and $V_{R} \in \mathbf{G}(S, x, R, \epsilon)$.

Then

$$
d_{H}\left(V_{r} \cap \boldsymbol{B}(0,1), V_{R} \cap \boldsymbol{B}(0,1)\right) \leq \epsilon\left(1+2 R r^{-1}\right) .
$$

Proof. We put $\lambda=r R^{-1}$ and we infer from Lemma 2.3 that $d_{\mathrm{H}}\left(\left(x+V_{R}\right) \cap\right.$ $\mathbf{B}(x, r), S \cap \mathbf{B}(x, r)) \leq 2 \epsilon \lambda^{-1} r$. Since also $d_{\mathrm{H}}\left(\left(x+V_{r}\right) \cap \mathbf{B}(x, r), S \cap \mathbf{B}(x, r)\right) \leq \epsilon r$, it follows from the triangle inequality for the Hausdorff distance, and from its invariance under translation, that $d_{\mathrm{H}}\left(V_{r} \cap \mathbf{B}(0, r), V_{R} \cap \mathbf{B}(0, r)\right) \leq \epsilon\left(1+2 \lambda^{-1}\right) r$.

2.5. Lemma (Different centers, same scale). Assume that

(1) $S \subset X, x_{1}, x_{2} \in S, \epsilon>0, \nu>1, R>0,0<\lambda<1,\left|x_{1}-x_{2}\right| \leq(1-\lambda) R$;

(2) $1-\lambda+\epsilon+\nu^{-1}<1$;

(3) $V_{i} \in \mathbf{G}\left(S, x_{i}, R, \epsilon\right), i=1,2$.

Then

$$
d_{H}\left(V_{1} \cap \boldsymbol{B}(0,1), V_{2} \cap \boldsymbol{B}(0,1)\right) \leq 3 \epsilon \nu .
$$

Proof. We let the relation $R=\nu r$ define $r>0$. Choose $\hat{\epsilon}>\epsilon$ such that $1-\lambda+\hat{\epsilon}+$ $\nu^{-1}<1$. Let $h_{1} \in V_{1} \cap \mathbf{B}(0, r)$ and define $\xi_{1}:=x_{1}+h_{1} \in\left(x_{1}+V_{1}\right) \cap \mathbf{B}\left(x_{1}, r\right) \subset$ $\left(x_{1}+V_{1}\right) \cap \mathbf{B}\left(x_{1}, R\right)$. Choose $y \in S \cap \mathbf{B}\left(x_{1}, R\right)$ such that $\left|y-\xi_{1}\right| \leq \hat{\epsilon} R$, according to hypothesis (3). Observe that

$$
\left|y-x_{2}\right| \leq\left|y-\xi_{1}\right|+\left|\xi_{1}-x_{1}\right|+\left|x_{1}-x_{2}\right| \leq \hat{\epsilon} R+\nu^{-1} R+(1-\lambda) R \leq R
$$

and define $\xi_{2}:=x_{2}+\pi_{V_{2}}\left(y-x_{2}\right)$. Since $y \in S \cap \mathbf{B}\left(x_{2}, R\right)$ it follows from assumption (3) that $\left|y-\xi_{2}\right| \leq \epsilon R$. Define also $c_{2}:=x_{2}+\pi_{V_{2}}\left(x_{1}-x_{2}\right)$. Since $x_{2} \in S \cap \mathbf{B}\left(x_{1},(1-\right.$

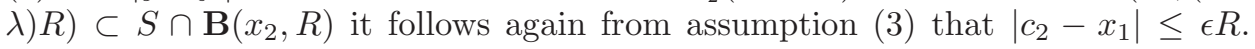


Finally we let $h_{2}:=\xi_{2}-c_{2} \in V_{2}$. Since $\left|h_{2}\right|=\left|\pi_{V_{2}}\left(y-x_{1}\right)\right| \leq\left|y-x_{1}\right| \leq r$ one has $h_{2} \in V_{2} \cap \mathbf{B}(0, r)$. Furthermore,

$$
\begin{aligned}
\left|h_{1}-h_{2}\right| & =\left|\xi_{1}-x_{1}-\xi_{2}+c_{2}\right| \\
& \leq\left|\xi_{1}-y\right|+\left|y-\xi_{2}\right|+\left|x_{1}-c_{2}\right| \\
& \leq \hat{\epsilon} R+\epsilon R+\epsilon R \\
& \leq 3 \hat{\epsilon} \nu r
\end{aligned}
$$

so that

$$
V_{1} \cap \mathbf{B}(0, r) \subset \mathbf{B}\left[V_{2} \cap \mathbf{B}(0, r), 3 \hat{\epsilon} \nu r\right] .
$$

The conclusion follows from the arbitrariness of $\hat{\epsilon}>\epsilon$ and Lemma 2.1(2).

2.2. Jacobians in Hilbert space. Let $V \in \mathbf{G}(X, m)$ be an $m$-plane. For a linear map $L: V \rightarrow V^{\perp}$ we define $\mathcal{J} L$ to be the non-negative number such that

$$
1+(\mathcal{J} L)^{2}=\operatorname{det}\left(\operatorname{id}_{V}+L^{*} L\right)=\operatorname{det}\left(\bar{L}^{*} \bar{L}\right)
$$

where $\bar{L}=\operatorname{id}_{V}+L: V \rightarrow X$ is the direct sum of $\operatorname{id}_{V}$ and $L$. If $\left(e_{1}, \ldots, e_{m}\right)$ is an orthonormal basis of $V$, we can define the matrix $M_{i j}:=\left\langle L e_{i}, L e_{j}\right\rangle$ and for any $K \subset\{1, \ldots, m\}$ we denote by $M_{K}$ the submatrix of $M$ corresponding to the indices in $K$. The determinant in question calculates as

$$
\begin{aligned}
1+(\mathcal{J} L)^{2} & =\operatorname{det}\left(\delta_{i j}+M_{i j}\right) \\
& =\sum_{\sigma \in S_{m}}(-1)^{|\sigma|} \prod_{i=1}^{m}\left(\delta_{i \sigma(i)}+M_{i \sigma(i)}\right) \\
& =1+\sum_{K \neq \emptyset} \operatorname{det}\left(M_{K}\right) .
\end{aligned}
$$

The Hilbert-Schmidt norm of $L$ is given by

$$
\|L\|_{\mathrm{HS}}:=\operatorname{tr}\left(L^{*} L\right)^{\frac{1}{2}} .
$$

The following identity relates the Hilbert-Schmidt norm to $\mathcal{J} L$,

$$
(\mathcal{J} L)^{2}=\|L\|_{\mathrm{HS}}^{2}+\sum_{\# K \geq 2} \operatorname{det}\left(M_{K}\right) .
$$

Further, if $0 \leq \lambda_{1}^{2} \leq \cdots \leq \lambda_{m}^{2}$ are the eigenvalues of $L^{*} L$ and $\|L\|$ is the usual operator norm of $L$, then

$$
\operatorname{Lip}(L)=\|L\|=\lambda_{m} \leq\left(\lambda_{1}^{2}+\cdots+\lambda_{m}^{2}\right)^{\frac{1}{2}}=\|L\|_{\mathrm{HS}} \leq \sqrt{m}\|L\| .
$$

If $f: A \subset V \rightarrow V^{\perp}$ is a map that is differentiable at $x \in A$ we also use the abbreviation $\mathcal{J} f_{x}$ instead of $\mathcal{J} D f_{x}$.

2.3. Chains, Slicing and tangent planes. With $\mathscr{P}_{m}(X ; G)$ and $\mathscr{R}_{m}(X ; G)$ we denote the $m$-dimensional polyhedral, respectively rectifiable, chains in the Hilbert space $X$ with coefficients in $G$. We refer to [13] and [14] for precise definitions and properties of these spaces. Here is a sketch and some basic results we will use later on. Consider a rectifiable $G$-chain $T \in \mathscr{R}_{m}(X ; G)$. According to the definition, $T$ can be represented by a sequence of bi-Lipschitz maps $\gamma_{i}: K_{i} \rightarrow X$ defined on compact sets $K_{i} \subset \mathbb{R}^{m}$ and measurable maps $\mathbf{g}_{i}: K_{i} \rightarrow G$ such that $\gamma_{i}\left(K_{i}\right) \cap \gamma_{j}\left(K_{j}\right)=\emptyset$ for $i \neq j$. The set $M_{T}:=\bigcup_{i} \gamma_{i}\left(K_{i}\right)$ is $\mathscr{H}^{m}$-rectifiable and $\mathrm{g}: X \rightarrow G$ is defined by $\mathbf{g}(x):=0_{G}$ for $x \notin M_{T}$ and $\mathbf{g}(x):=\mathbf{g}_{i}\left(\gamma_{i}^{-1}(x)\right)$ if $x \in \gamma_{i}\left(K_{i}\right)$. Clearly, $\mathbf{g}$ is $\mathscr{H}^{m}$-measurable and it is further assumed to be integrable, 
respectively that the corresponding measure $\|T\|:=\mathscr{H}^{m}\llcorner\|\mathrm{~g}\|$ is finite. The mass of $T$ is the total measure $\mathbf{M}(T)=\|T\|(X)$. For any Borel set $B \subset X$, the restriction $T\left\llcorner B\right.$ is well defined by the weight function $\chi_{B} \mathbf{g}: X \rightarrow G$. Obviously,

$$
\mathbf{M}\left(T\llcorner B)=\int_{B \cap M_{T}}\|\mathbf{g}(x)\| d \mathscr{H}^{m}(x) .\right.
$$

Since the metric differential (in the sense of Kirchheim [16]) of each $\gamma_{i}$ is represented by a scalar product at almost every point, we can further assume that the $\gamma_{i}$ are close to isometries, i.e. given a $\lambda>1$ we can find parametrizations $\gamma_{i}$ as above such that $\max \left\{\operatorname{Lip}\left(\gamma_{i}\right), \operatorname{Lip}\left(\gamma_{i}^{-1}\right)\right\} \leq \lambda$, see [16, Lemma 4] and [15, Lemma 3.2.2].

The $m$-density of $\|T\|$ at a point $x$ is defined by

$$
\Theta^{m}(\|T\|, x):=\lim _{r \downarrow 0} \frac{\|T\|(\mathbf{B}(x, r))}{\boldsymbol{\alpha}(m) r^{m}},
$$

in case the limit exists. It can be shown that for $\|T\|$-a.e. point $x \in X$, there holds $\Theta^{m}(\|T\|, x)=\|\mathbf{g}(x)\|$.

Let $f: X \rightarrow \mathbb{R}^{n}$ be a Lipschitz map for some $n \leq m$. Following [13, Section 3.7], the slice $\langle T, f, y\rangle$ is an element of $\mathscr{R}_{m-n}(X ; G)$ for almost every $y \in \mathbb{R}^{n}$. In particular, for almost every $y$, the set $M_{T} \cap f^{-1}\{y\}$ is $\mathscr{H}^{m-n}$-rectifiable and has a $G$-orientation given by $\pm \mathrm{g}$. On a given chart $K_{i}$ define $T_{i}$ as the $m$-dimensional $G$-chain equipped with $G$-orientation $\mathbf{g}_{i}$. For almost all $y$ it follows from [13, Theorem 3.8.1] that

$$
\langle T, f, y\rangle\left\llcorner\gamma_{i}\left(K_{i}\right)=\left\langle T\left\llcorner\gamma_{i}\left(K_{i}\right), f, y\right\rangle=\gamma_{i \#}\left\langle T_{i}, f \circ \gamma_{i}, y\right\rangle\right.\right.
$$

Hence by the coarea formula [15, Theorem 3.2.12],

$$
\begin{aligned}
\int_{\mathbb{R}^{n}} \mathbf{M}(\langle T, f, y\rangle & \left\llcorner\gamma_{i}\left(K_{i}\right)\right) d y \\
& =\int_{\mathbb{R}^{n}} \mathbf{M}\left(\gamma_{i \#}\left\langle T_{i}, f \circ \gamma_{i}, y\right\rangle\right) d y \\
& \leq \operatorname{Lip}\left(\gamma_{i}\right)^{m-n} \int_{\mathbb{R}^{n}} \mathbf{M}\left(\left\langle T_{i}, f \circ \gamma_{i}, y\right\rangle\right) d y \\
& =\operatorname{Lip}\left(\gamma_{i}\right)^{m-n} \int_{\mathbb{R}^{n}} \int_{K_{i} \cap\left(f \circ \gamma_{i}\right)^{-1}\{y\}}\left\|\mathbf{g}_{i}(z)\right\| d \mathscr{H}^{m-n}(z) d y \\
& =\operatorname{Lip}\left(\gamma_{i}\right)^{m-n} \int_{K_{i}}\left\|\mathbf{g}_{i}(x)\right\| C_{n}\left(D\left(f \circ \gamma_{i}\right)_{x}\right) d x,
\end{aligned}
$$

where $C_{n}(L)$ denotes the coarea factor of a linear map $L: \mathbb{R}^{m} \rightarrow \mathbb{R}^{n}$. In $[5$, Lemma 9.3] or the proof of [15, Lemma 3.2.10] it is shown that in case the kernel $K$ of $L$ has dimension $m-n$, then $C_{n}(L)=J_{m}(q) / J_{m-n}\left(\left.p\right|_{K}\right)$ (otherwise $C_{n}(L)=0$ ), where $q=L+p: \mathbb{R}^{m} \rightarrow \mathbb{R}^{m}$ is linear and $p$ is injective on $K$. In the setting of Hilbert spaces we have $J_{k}(q) J_{k^{\prime}}\left(q^{\prime}\right)=J_{k+k^{\prime}}\left(q \oplus q^{\prime}\right)$ in case $q: W \rightarrow \mathbb{R}^{k}, q^{\prime}: W^{\prime} \rightarrow \mathbb{R}^{k^{\prime}}$ and $W \oplus W^{\prime}$ is a direct sum of Hilbert spaces of dimension $k$ and $k^{\prime}$ respectively. Hence, $C_{n}(L)=J_{n}\left(\left.L\right|_{K^{\perp}}\right)$ and in particular $C_{n}(L) \leq\|L\|^{n}=\operatorname{Lip}(L)^{n}$. Applied to 
the situation above and assuming that $\max \left\{\operatorname{Lip}\left(\gamma_{i}\right), \operatorname{Lip}\left(\gamma_{i}^{-1}\right)\right\} \leq \lambda$ we get

$$
\begin{aligned}
\int_{\mathbb{R}^{n}} \mathbf{M}\left(\langle T, f, y\rangle\left\llcorner\gamma_{i}\left(K_{i}\right)\right) d y\right. & \leq \lambda^{m-n} \operatorname{Lip}\left(f \circ \gamma_{i}\right)^{n} \int_{K_{i}}\left\|\mathbf{g}_{i}(x)\right\| d x \\
& \leq \lambda^{m} \operatorname{Lip}(f)^{n} \mathbf{M}\left(\gamma_{i}^{-1} \#\left(T\left\llcorner\gamma_{i}\left(K_{i}\right)\right)\right)\right. \\
& \leq \lambda^{2 m} \operatorname{Lip}(f)^{n} \mathbf{M}\left(T\left\llcorner\gamma_{i}\left(K_{i}\right)\right) .\right.
\end{aligned}
$$

Summing over all $i$ and taking the limit for $\lambda \rightarrow 1$ we get

$$
\int_{\mathbb{R}^{n}} \mathbf{M}(\langle T, f, y\rangle) d y \leq \operatorname{Lip}(f)^{n} \mathbf{M}(T) .
$$

Another important tool we need is the cone construction. For a rectifiable chain $T \in \mathscr{R}_{m-1}(X ; G)$ one can construct the cone over $T$ with center $x \in X, \llbracket x \rrbracket \mathbb{*} \in$ $\mathscr{R}_{m}(X ; G)$. In case $T$ is a polyhedral chain we can write $T=\sum_{i} g_{i} \llbracket S_{i} \rrbracket$ for finitely many oriented simplices $S_{i}$. If $x=0$, then $\llbracket 0 \rrbracket \nVdash T=\sum_{i} g_{i} \llbracket S_{i}^{\prime} \rrbracket$ where $S_{i}^{\prime}:=\{t x$ : $\left.t \in[0,1], x \in S_{i}\right\}$ and an appropriate orientation. For general rectifiable chains we may define $\llbracket 0 \rrbracket \mathbb{*} T:=\psi_{\#}(\llbracket 0,1 \rrbracket \times T)$ where $\psi(t, x)=t x$. If $B \subset \partial \mathbf{B}(0, r)$ has $\mathscr{H}^{m-1}$-finite measure, then the set $B^{\prime}=\{t x: t \in[0,1], x \in B\}$ satisfies $\mathscr{H}^{m}\left(B^{\prime}\right)=\frac{r}{m} \mathscr{H}^{m-1}(B)$ as one can verify easily. Hence if $\operatorname{spt}(T) \subset \partial \mathbf{B}(0, r)$ and $\partial T=0$, then $\partial(\llbracket 0 \rrbracket \nVdash T)=T$ and

$$
\mathbf{M}(\llbracket 0 \rrbracket * T)=\frac{r}{m} \mathbf{M}(T) .
$$

In case $B \subset X \backslash \mathbf{U}(0, r)$ we get $\mathscr{H}^{m}\left(B^{\prime} \cap \mathbf{B}(0, r)\right) \leq \frac{r}{m} \mathscr{H}^{m-1}(B)$ since the orthogonal projection of $X \backslash \mathbf{B}(0, r)$ onto $\partial \mathbf{B}(0, r)$ is 1-Lipschitz. Hence if $\operatorname{spt}(T) \subset$ $X \backslash \mathbf{U}(0, r)$ and $\partial T=0$, then $\operatorname{spt}(\partial(\llbracket 0 \rrbracket \mathbb{x} T)) \subset X \backslash \mathbf{U}(0, r)$ and

$$
\mathbf{M}(\llbracket 0 \rrbracket \times T) \leq \frac{r}{m} \mathbf{M}(T) .
$$

The basic idea in order to establish a regularity result for some chain $T$ is to show that $\operatorname{spt}(T)$ can be uniformly approximated by planes. Rectifiable measures in $\mathbb{R}^{n}$ have weak tangent planes almost everywhere. It is then a classical observation that this tangent plane is actually a tangent plane for the support of the measure in case the measure is Ahlfors regular. Here we show this fact for chains in $X$. For this it is crucial that Hilbert spaces have the Radon-Nikodým property, which allows to differentiate Lipschitz maps $\mathbb{R}^{m} \rightarrow X$ at almost every point. For a Radon measure $\phi$ in $X$ and $V \in \mathbf{G}(X, m)$ we define

$$
\beta_{\infty}(\phi, x, r, V):=r^{-1} \sup \left\{\left|\pi_{V^{\perp}}(y-x)\right|: y \in \operatorname{spt}(\phi) \cap \mathbf{B}(x, r)\right\} .
$$

2.6. Lemma. Let $X$ be a Hilbert space, $T \in \mathscr{R}_{m}(X ; G)$ and let $U \subset \operatorname{spt}(T)$ be a relatively open set with $\operatorname{dist}(U, \operatorname{spt}(\partial T)) \geq r_{0}>0$. Assume that there are constants $c_{2} \geq c_{1}>0$ such that $\|T\|$ is Ahlfors regular in $U$ in the sense that for all $x \in U$ and $0<r<r_{0}$,

$$
c_{1} \boldsymbol{\alpha}(m) r^{m} \leq\|T\|(\boldsymbol{B}(x, r)) \leq c_{2} \boldsymbol{\alpha}(m) r^{m} .
$$

Then for $\|T\|$-a.e. $x \in U$ there is a plane $W_{x} \in \mathbf{G}(X, m)$ such that

$$
\lim _{r \downarrow 0} \beta_{\infty}\left(\|T\|, x, r, W_{x}\right)=0 .
$$

Moreover, there is some $r_{x}>0$ such that for all $0<r<r_{x}$, there holds $\beta_{\infty}\left(\|T\|, x, r, W_{x}\right) \leq 2^{-1}$ and

$$
\pi_{W_{x} \#}\left(T\left\llcorner\left(B(x, r) \cap Z_{W_{x}}\left(x, 2^{-1} r\right)\right)\right)=g_{x} \llbracket \boldsymbol{B}_{W_{x}}\left(\pi_{W_{x}}(x), 2^{-1} r\right) \rrbracket,\right.
$$


for some $g_{x} \in G$ with $\left\|g_{x}\right\|=\Theta^{m}(\|T\|, x)$.

Proof. The bounds on volumes of balls (2.10) imply that $c_{1} \leq \Theta^{m *}(\|T\|, x) \leq c_{2}$ for all $x \in U$ and by a standard result of measure theory

$$
c_{1} \mathscr{H}^{m}(B) \leq\|T\|(B) \leq 2^{m} c_{2} \mathscr{H}^{m}(B)
$$

for all Borel measurable subsets $B \subset U$, see e.g. [7, Theorem 2.4.3]. As in Subsection 2.3 let $\gamma^{i}: K_{i} \rightarrow U$ be countably many bi-Lipschitz maps defined on compact sets $K_{i} \subset \mathbb{R}^{m}$ with pairwise disjoint images and $\mathscr{H}^{m}\left(U \backslash \bigcup_{i} \gamma^{i}\left(K_{i}\right)\right)=0$. Let $\mathbf{g}_{i}: K_{i} \rightarrow G$ be $\mathscr{H}^{m}$-measurable functions such that $T\left\llcorner U=\sum_{i=1}^{\infty} \gamma_{\#}^{i}\left(\mathbf{g}_{i} \llbracket K_{i} \rrbracket\right)\right.$ and $\left\|\mathbf{g}_{i}\right\| \in\left[c_{1}, c_{2}\right]$. The $\mathscr{H}^{m}$-measurable function $\mathbf{g}: X \rightarrow G$ is defined to be $\mathbf{g}_{i} \circ\left(\gamma^{i}\right)^{-1}$ on $\gamma^{i}\left(K_{i}\right)$ and equal to $0_{G}$ on the complement of the union of all these sets. We can further assume that for each $i$ the inverse of $\gamma^{i}$ is extended to a Lipschitz map $\psi^{i}: X \rightarrow \mathbb{R}^{m}$. Kirszbraun's Theorem allows to extend $\gamma^{i}$ to a Lipschitz map $\gamma^{i}: \mathbb{R}^{m} \rightarrow X$. In what follows we don't need a sharp control on the Lipschitz constant of these extensions and just assume that $\operatorname{Lip}\left(\gamma^{i}\right), \operatorname{Lip}\left(\psi^{i}\right) \leq L$ for some $L>1$. The extended maps $\gamma^{i}$ are differentiable almost everywhere since Hilbert spaces have the Radon-Nikodým property. For a proof of this see for example [12, 1.2]. The Radon-Nikodým property implies as in a standard proof of Rademacher's Theorem that each $\gamma^{i}$ is differentiable at almost every point of $\mathbb{R}^{m}$. With the help of the area formula we see that for $\mathscr{H}^{m}$-almost every point $x \in U$ there is an index $i$ and $y \in K_{i}$ such that

(1) $\gamma^{i}(y)=x$,

(2) $D \gamma_{y}^{i}$ exists and has rank $m$,

(3) $\Theta^{m}\left(\mathscr{H}^{m}\left\llcorner K_{i}, y\right)=\Theta^{m}\left(\mathscr{H}^{m}\left\llcorner\gamma^{i}\left(K_{i}\right), x\right)=\Theta^{m}\left(\mathscr{H}^{m}\llcorner U, x)=1\right.\right.\right.$,

(4) $\left\|\mathbf{g}_{i}\right\|: K_{i} \rightarrow \mathbb{R}$ has an approximate point of continuity at $y$.

Let $W_{x} \in \mathbf{G}(X, m)$ be the image of $D \gamma_{y}^{i}$. The differentiability of $\gamma^{i}$ at $y$ and the fact that $\gamma^{i}$ is $L$-bi-Lipschitz on $K_{i}$ imply that for any $0<\epsilon<\frac{1}{4}$ there is a $0<r_{\epsilon}<r_{0}$ such that $\mathbf{B}\left(x, r_{\epsilon}\right) \cap \operatorname{spt}(T) \subset U$ and for all $0<r<r_{\epsilon}$,

$$
\operatorname{dist}\left(\gamma^{i}(\mathbf{B}(y, L r)), x+W_{x}\right) \leq \epsilon r \text {, and } \gamma^{i}\left(K_{i} \backslash \mathbf{B}(y, L r)\right) \subset U \backslash \mathbf{B}(x, r),
$$

and with (3),

$$
\begin{aligned}
\left(1-3^{-1}\left(2^{m} c_{2}\right)^{-1} c_{1} \epsilon^{m}\right) \boldsymbol{\alpha}(m) r^{m} & \leq \mathscr{H}^{m}\left(\gamma^{i}\left(K_{i}\right) \cap \mathbf{B}(x, r)\right) \\
& \leq \mathscr{H}^{m}(U \cap \mathbf{B}(x, r)) \\
& \leq\left(1+3^{-1}\left(2^{m} c_{2}\right)^{-1} c_{1} \epsilon^{m}\right) \boldsymbol{\alpha}(m) r^{m} .
\end{aligned}
$$

The inclusions in (2.12) imply

$$
\gamma^{i}\left(K_{i}\right) \cap \mathbf{B}(x, r) \subset N(x, \epsilon r),
$$

where $N(x, t):=\left\{z \in U: \operatorname{dist}\left(z, x+W_{x}\right) \leq t\right\}$ for $t \geq 0$. Assume by contradiction that for some $0<r<r_{\epsilon}$ there is a point

$$
x^{\prime} \in U \cap \mathbf{B}(x, r / 2) \backslash N(x, 2 \epsilon r) .
$$

Then obviously $\mathbf{B}\left(x^{\prime}, \epsilon r\right) \subset \mathbf{B}(x, r)$ and $\operatorname{dist}\left(\mathbf{B}\left(x^{\prime}, \epsilon r\right), x+W_{x}\right)>\epsilon r$ as well as $\mathscr{H}^{m}\left(U \cap \mathbf{B}\left(x^{\prime}, \epsilon r\right)\right) \geq\left(2^{m} c_{2}\right)^{-1} c_{1} \boldsymbol{\alpha}(m)(\epsilon r)^{m}$ by (2.10) and (2.11). Hence with the first estimate of (2.13) and (2.14),

$$
\begin{aligned}
\mathscr{H}^{m}(U \cap \mathbf{B}(x, r)) & \geq \mathscr{H}^{m}\left(\gamma^{i}\left(K_{i}\right) \cap \mathbf{B}(x, r)\right)+\mathscr{H}^{m}(U \backslash N(x, \epsilon r)) \\
& >\left(1+3^{-1}\left(2^{m} c_{2}\right)^{-1} c_{1} \epsilon^{m}\right) \boldsymbol{\alpha}(m) r^{m} .
\end{aligned}
$$


This contradicts the last estimate of (2.13). Thus (2.15) is wrong and $\lim \sup _{r \downarrow 0} \beta_{\infty}\left(\|T\|, x, r, W_{x}\right) \leq 4 \epsilon$ for all $\epsilon>0$. This proves the first statement.

To establish the statement about the projections, let $r_{x}:=2^{-1} r_{8^{-1}}$. Then $\boldsymbol{\beta}_{\infty}\left(\|T\|, x, r, W_{x}\right) \leq 2^{-1}$ for all $0<r<r_{x}$. For almost every such $r,\left\langle T, d_{x}, r\right\rangle=$ $\partial\left(T\llcorner\mathbf{B}(x, r)) \in \mathscr{R}_{m-1}(X ; G)\right.$, where $d_{x}(z)=|x-z|$. Since $\operatorname{spt}\left(\left\langle T, d_{x}, r\right\rangle\right) \subset$ $\partial \mathbf{B}(x, r) \cap N\left(x, 2^{-1} r\right)$ we get that $\left|\pi_{W_{x}}(z-x)\right|>2^{-1} r$ for all $z \in \operatorname{spt}\left(\left\langle T, d_{x}, r\right\rangle\right)$. Hence the constancy theorem [14, Theorem 6.4] implies that for $0<s \leq 2^{-1} r$,

$$
\pi_{W_{x} \#}\left(T \left\llcorner\left(\mathbf{B}(x, r) \cap Z_{W_{x}}(x, s)\right)=\left(\pi _ { W _ { x } \# } ( T \llcorner \mathbf { B } ( x , r ) ) ) \left\llcorner\mathbf{B}\left(\pi_{W_{x}}(x), s\right),\right.\right.\right.\right.
$$

and this chain is equal to $g_{r, s} \llbracket \mathbf{B}\left(\pi_{W_{x}}(x), s\right) \rrbracket$ for some $g_{r, s} \in G$. The map $(r, s) \mapsto$ $g_{r, s}$ is locally constant by the constancy theorem and since the domain $\{(r, s) \in$ $\left.\left(0, r_{x}\right) \times\left(0, r_{x}\right): 2 s \leq r\right\}$ is connected, this function is constant. Therefore we find a unique $g_{x} \in G$ as in the second statement of the lemma. It remains to show that $\Theta^{m}(\|T\|, x)=\left\|g_{x}\right\|$.

Since $\|T\|(\mathbf{B}(x, r))=\sum_{j} \int_{\mathbf{B}(x, r) \cap \gamma^{j}\left(K_{j}\right)}\left\|\mathbf{g}_{j} \circ\left(\gamma^{j}\right)^{-1}\right\| d \mathscr{H}^{m}$ and $\left\|\mathbf{g}_{j}\right\| \in\left[c_{1}, c_{2}\right]$, it follows directly from (3) and (4) that $\|\mathbf{g}\|$ is $\mathscr{H}^{m}$-approximately continuous at $x$ and

$$
\Theta^{m}(\|T\|, x)=\Theta^{m}\left(\|T\|\left\llcorner\gamma^{i}\left(K_{i}\right), x\right)=\|\mathbf{g}(x)\| .\right.
$$

Consider the $L$-Lipschitz function $f: X \rightarrow \mathbb{R}$ given by

$$
f(z):=\max \left\{\left|\psi^{i}(z)-\psi^{i}(x)\right|, L^{-1}|z-x|\right\} .
$$

If $z \in \gamma^{i}\left(K_{i}\right)$, then $f(z)=\left|\psi^{i}(z)-\psi^{i}(x)\right|$ and also

$$
\mathbf{B}\left(x, L^{-1} r\right) \subset\{f \leq r\} \subset \mathbf{B}(x, L r) .
$$

As before, $\langle T, f, r\rangle=\partial\left(T\llcorner\{f \leq r\}) \in \mathscr{R}_{m-1}(X ; G)\right.$ for almost every $r>0$. With (3) and the coarea formula we find a sequence $r_{k} \in\left[2^{-k-1}, 2^{-k}\right]$ such that

$$
\lim _{k \rightarrow \infty} \frac{\mathscr{H}^{m-1}\left(\partial \mathbf{B}\left(y, r_{k}\right) \cap K_{i}\right)}{\mathscr{H}^{m-1}\left(\partial \mathbf{B}\left(y, r_{k}\right)\right)}=1,
$$

as well as $\left\langle T, f, r_{k}\right\rangle=\partial\left(T\left\llcorner\left\{f \leq r_{k}\right\}\right) \in \mathscr{R}_{m-1}(X ; G)\right.$ for all $k$. From this it follows that $\frac{1}{r_{k}} d_{\mathrm{H}}\left(\partial \mathbf{B}\left(y, r_{k}\right) \cap K_{i}, \partial \mathbf{B}\left(y, r_{k}\right)\right) \rightarrow 0$. Because $\gamma^{i}: \mathbb{R}^{m} \rightarrow X$ is Lipschitz, differentiable at $y$ and $\lim _{r \downarrow 0} \beta_{\infty}\left(\|T\|, x, r, W_{x}\right)=0$, it follows from (3) that $\frac{1}{r_{k}} \operatorname{dist}\left(\operatorname{spt}\left(\left\langle T, f, r_{k}\right\rangle\right), \gamma^{i}\left(K_{i}\right)\right) \rightarrow 0$ and further $\frac{1}{r_{k}} d_{\mathrm{H}}\left(\gamma^{i}\left(\partial \mathbf{B}\left(y, r_{k}\right) \cap\right.\right.$ $\left.\left.K_{i}\right), \operatorname{spt}\left(\left\langle T, f, r_{k}\right\rangle\right)\right) \rightarrow 0$. Thus

$$
\frac{1}{r_{k}} d_{\mathrm{H}}\left(\partial \mathbf{B}\left(y, r_{k}\right), \operatorname{spt}\left(\partial T_{k}\right)\right) \rightarrow 0,
$$

for the push-forward $T_{k}:=\psi_{\#}^{i}\left(T\left\llcorner\left\{f \leq r_{k}\right\}\right) \in \mathscr{R}_{m}\left(\mathbb{R}^{m} ; G\right)\right.$. The constancy theorem implies now that there are $g_{k} \in G$ and a sequence $0<s_{k}<r_{k}$ with $s_{k} / r_{k} \rightarrow 1$ and

$$
T_{k}\left\llcorner\mathbf{B}\left(y, s_{k}\right)=g_{k} \llbracket \mathbf{B}\left(y, s_{k}\right) \rrbracket .\right.
$$

From the construction of the push-forward as in $[13,3.5]$, it follows that

$$
T_{k}=\mathbf{g}_{i} \llbracket K_{i} \cap \mathbf{B}\left(y, r_{k}\right) \rrbracket+\psi_{\#}^{i}\left(T\left\llcorner A_{k}\right),\right.
$$

for some $\mathscr{H}^{m}$-measurable set $A_{k} \subset U \backslash \gamma^{i}\left(K_{i}\right)$. From (3) and (2.16) it follows that $\frac{1}{r_{k}^{m}} \mathscr{H}^{m}\left(\psi^{i}\left(A_{k}\right)\right) \rightarrow 0$ and also $\frac{1}{r_{k}^{m}} \mathbf{M}\left(\psi_{\#}^{i}\left(T\left\llcorner A_{k}\right)\right) \rightarrow 0\right.$. With (2.17) we conclude that $\mathbf{g}_{i}=g_{k}$ on $\mathbf{B}\left(y, r_{k} / 2\right) \backslash \psi^{i}\left(A_{k}\right)$. Since $r_{k} \in\left[2^{-k-1}, 2^{-k}\right]$, this shows that $g_{k}=g_{k+1}$ if $k$ is large enough. Hence $\mathbf{g}_{i}$ is approximately equal to some $g_{x}^{\prime} \in G$ 
at $y \in K_{i}$ with $\left\|g_{x}^{\prime}\right\|=\|\mathbf{g}(x)\|=\Theta^{m}(\|T\|, x)$ by (4) and (2.16). It follows that $\frac{1}{r_{k}^{m}} \mathbf{M}\left(T_{k}-g_{x}^{\prime} \llbracket \mathbf{B}\left(y, r_{k}\right) \rrbracket\right) \rightarrow 0$. Thus, $\frac{1}{r_{k}^{m}} \mathbf{M}\left(T\left\llcorner\left\{f \leq r_{k}\right\}-\gamma_{\#}^{i}\left(g_{x}^{\prime} \llbracket \mathbf{B}\left(y, r_{k}\right) \rrbracket\right)\right) \rightarrow 0\right.$ and further

$$
\frac{1}{r_{k}^{m}} \mathbf{M}\left(\pi_{W_{x} \#}\left(T\left\llcorner\left\{f \leq r_{k}\right\}\right)-\left(\pi_{W_{x}} \circ \gamma^{i}\right)_{\#}\left(g_{x}^{\prime} \llbracket \mathbf{B}\left(y, r_{k}\right) \rrbracket\right)\right) \rightarrow 0 .\right.
$$

From the fact that $\pi_{W_{x}} \circ \gamma^{i}$ is differentiable at $y$ and (2.17),

$$
\mathbf{M}\left(g_{x}^{\prime} \llbracket x^{\prime}+D\left(\pi_{W_{x}} \circ \gamma^{i}\right)_{y}(\mathbf{B}(0,1)) \rrbracket-\left(\eta_{r_{k}} \circ \pi_{W_{x}} \circ \gamma^{i}\right)_{\#}\left(g_{x}^{\prime} \llbracket \mathbf{B}\left(y, r_{k}\right) \rrbracket\right)\right) \rightarrow 0,
$$

for $k \rightarrow \infty$, where $\eta_{r}(z):=\frac{1}{r}\left(z-x^{\prime}\right)+x^{\prime}$ and $x^{\prime}=\pi_{W_{x}}(x)$. We already know that $\pi_{W_{x}}$ projects $T$ around $x$ to a chain with weight $g_{x}$, hence $g_{x}=g_{x}^{\prime}$ follows from (2.18) and (2.19). This concludes the proof.

The following lemma shows that the group element associated with a projection doesn't change for projections to nearby planes. This is a direct consequence of the constancy theorem.

2.7. Lemma. Let $T \in \mathscr{R}_{m}(X ; G)$ be rectifiable chain. Assume that there is an $m$-plane $V \in \mathbf{G}(X, m)$ such that,

(1) $\operatorname{spt}(T) \subset \boldsymbol{B}(0,1)$ and $\operatorname{spt}(\partial T) \subset \partial \boldsymbol{B}(0,1)$,

(2) $\left|\pi_{V^{\perp}}(x)\right| \leq \frac{1}{5}$ for $x \in \operatorname{spt}(T)$,

(3) $\pi_{V \#}\left(T\left\llcorner Z_{V}\left(0, \frac{1}{2}\right)\right)=g_{0} \llbracket \boldsymbol{B}_{V}\left(0, \frac{1}{2}\right) \rrbracket\right.$.

Then for any $W \in \mathbf{G}(X, m)$ that satisfies $\left\|\pi_{W}-\pi_{V}\right\| \leq \frac{1}{5}$,

$$
\pi_{W \#}\left(T\left\llcorner Z_{W}\left(0,2^{-1}\right)\right)=g_{0} \llbracket \boldsymbol{B}_{W}\left(0,2^{-1}\right) \rrbracket .\right.
$$

We assume that $W$ and $V$ are given orientations such that the projection $\pi_{W}$ : $W \rightarrow V$ is orientation preserving.

Proof. There is a Lipschitz family of $m$-planes $t \mapsto W_{t}$ connecting $V$ with $W$ such that $\left\|\pi_{W_{t}}-\pi_{W_{s}}\right\| \leq L|s-t|$ and $\left|\pi_{V}(w)-w\right| \leq 5^{-1}|w|$ for all $s, t \in[0,1]$ and $w \in W_{t}$. With Lemma 2.1, the latter is equivalent to $\left\|\pi_{V}-\pi_{W_{t}}\right\| \leq 5^{-1}$ for all $t$. Such a family of planes is for example given by $\operatorname{im}\left(L_{t}\right)$ where $L_{t}: W \rightarrow X$ is defined by $L_{t}(w):=\pi_{V}(w)+t \pi_{V^{\perp}}(w)$. With a reparametrization, we may assume that $W_{t}=V, W_{1-t}=W$ for $t \leq 4^{-1}$. Let $S:=\llbracket 0,1 \rrbracket \times T \in \mathscr{R}_{m+1}(\mathbb{R} \times X ; G)$ and $\psi:[0,1] \times X \rightarrow[0,1] \times V$ be the Lipschitz map given by $\psi(t, x)=\left(t, \pi_{V} \circ \pi_{W_{t}}(x)\right)$. We identify $[0,1] \times V$ isometrically (in an orientation preserving way) with $[0,1] \times$ $\mathbb{R}^{m} \subset \mathbb{R}^{m+1}$. The boundary of $S$ is given by, see $[15,4.1 .8]$ and the definition of the boundary in [13, Section 5.1],

$$
\partial S=\llbracket 1 \rrbracket \times T-\llbracket 0 \rrbracket \times T-\llbracket 0,1 \rrbracket \times \partial T .
$$

Let $x \in \operatorname{spt}(T)$. By assumption, $x$ can be written as $x=v+v^{\perp}$ with $v \in \mathbf{B}_{V}(0,1)$ and $\left|v^{\perp}\right| \leq 5^{-1}$. It follows by the closeness of $W_{t}$ to $V$,

$$
\begin{aligned}
\left|\pi_{W_{t}^{\perp}}(x)\right| & =\left|\pi_{W_{t}}(x)-x\right| \leq\left|\pi_{W_{t}}(v)-v\right|+\left|\pi_{W_{t}}\left(v^{\perp}\right)-v^{\perp}\right| \\
& \leq \frac{|v|}{5}+\frac{2}{5} \leq \frac{3}{5} .
\end{aligned}
$$

In particular if $|x|=1$,

$$
\begin{aligned}
\left|\pi_{V} \circ \pi_{W_{t}}(x)\right| & \geq\left|\pi_{V}(x)\right|-\left|\pi_{V}\left(\pi_{W_{t}}(x)-x\right)\right| \geq 1-\left|\pi_{V^{\perp}}(x)\right|-\left|\pi_{W_{t}^{\perp}}(x)\right| \\
& \geq 1-\frac{4}{5} \geq \frac{1}{5}
\end{aligned}
$$


Fix some $t_{0} \in\left(0, \frac{1}{4}\right)$ and let $B:=\left(t_{0}, 1-t_{0}\right) \times \mathbf{U}_{V}\left(0, \frac{1}{5}\right)$. Because of $(2.20)$ and (2.22), the set $B$ lies outside the support of the boundary $\partial \psi_{\#} S$. The constancy theorem implies that $\left(\psi_{\#} S\right)\left\llcorner B=g \llbracket B \rrbracket\right.$ for some $g \in G$. For almost all $t \in\left[t_{0}, 1-t_{0}\right]$ we have for the projection $\pi(t, x):=t$,

$$
\left\langle\left(\psi_{\#} S\right)\llcorner B, \pi, t\rangle=\langle g \llbracket B \rrbracket, \pi, t\rangle=g\left(\llbracket t \rrbracket \times \llbracket \mathbf{U}_{V}\left(0,5^{-1}\right) \rrbracket\right) .\right.
$$

Considering $t_{0}<t<4^{-1}$ we infer $g=g_{0}$. The same slicing argument shows that

$$
\left(\left(\pi_{V} \circ \pi_{W_{t}}\right)_{\#} T\right)\left\llcorner\mathbf{U}_{V}\left(0,5^{-1}\right)=g_{0} \llbracket \mathbf{U}_{V}\left(0,5^{-1}\right) \rrbracket\right.
$$

for almost all $t \in\left[t_{0}, 1-t_{0}\right] . \pi_{V}: W_{t} \rightarrow V$ is bi-Lipschitz and orientation preserving (by assumption). Hence we get for some small $\rho>0\left(\operatorname{such}\right.$ that $\mathbf{U}_{W_{t}}(0, \rho) \subset$ $\left.\pi_{V}{ }^{-1}\left(\mathbf{U}_{V}\left(0, \frac{1}{5}\right)\right)\right)$,

$$
\left(\pi_{W_{t} \#} T\right)\left\llcorner\mathbf{U}_{W_{t}}(0, \rho)=\left(\pi_{W_{t} \#} T\right)\left\llcorner\mathbf{U}_{W_{t}}(0, \rho)=g_{0} \llbracket \mathbf{U}_{W_{t}}(0, \rho) \rrbracket .\right.\right.
$$

Since $\left(\frac{3}{5}\right)^{2}+\left(\frac{1}{2}\right)^{2}<1$ and $(2.21)$, we again see by the constancy theorem on $W$ that the conclusion of the lemma holds for $W=W_{1}$.

2.4. Excess over a plane. For a chain $T \in \mathscr{R}_{m}(X ; G)$, an $m$-plane $V \in \mathbf{G}(X, m)$ and an $\mathscr{H}^{m}$-measurable set $B \subset V$ the excess over $B$ is defined by

$$
\operatorname{Exc}(T, V, B):=\mathbf{M}\left(T\left\llcorner\pi_{V}^{-1}(B)\right)-\mathbf{M}\left(\pi_{V \#}\left(T\left\llcorner\pi_{V}^{-1}(B)\right)\right)\right.\right.
$$

Since $\operatorname{Lip}\left(\pi_{V}\right)=1$, this number is non-negative and for disjoint Borel measurable sets $B, B^{\prime} \subset V$ it is $\operatorname{Exc}(T, V, B)+\operatorname{Exc}\left(T, V, B^{\prime}\right)=\operatorname{Exc}\left(T, V, B \cup B^{\prime}\right)$. From this it follows that Exc is subadditive on the Borel $\sigma$-algebra on $V$. We also use the notation

$$
\operatorname{Exc}(T, V, x, r):=\operatorname{Exc}\left(T, V, \mathbf{B}_{V}\left(\pi_{V}(x), r\right)\right),
$$

and for $x$ at the origin we abbreviate $\operatorname{Exc}_{r}(T, V)$ for $\operatorname{Exc}(T, V, 0, r)$. We will occasionally calculate the excess for different masses. For example $\mathbf{S}(T)$ denotes the size of $T$ and is just the Hausdorff measure of the underlying set $M_{T}$. Alternatively, $\mathbf{S}(T)$ is the mass with respect to the discrete norm on $G$ taking only the values 0 and 1 . The excess with respect to $\mathbf{S}$ is denoted by $\operatorname{Exc}(T, V, B, \mathbf{S})$, and similarly if we work with another mass. Here we give some first implications of small excess. For a given $T \in \mathscr{R}_{m}(X ; G)$ and $V \in \mathbf{G}(X, m)$ we denote by $E_{2}$ the set of points $x \in \mathbf{B}_{V}(0,1)$ for which $M_{x}:=\pi_{V}{ }^{-1}\{x\} \cap M_{T}$ contains more than one point. One can show that this set is Lebesgue measurable and hence $E_{2}$ contains a Borel set $E_{2}^{\prime}$ with $\mathscr{L}^{m}\left(E_{2} \backslash E_{2}^{\prime}\right)=0$.

2.8. Lemma ([20, Lemma 5]). Let $T \in \mathscr{R}_{m}(X ; G)$ and assume there is an m-plane $V \in \mathbf{G}(X, m)$ such that $\operatorname{Exc}_{1}(T, V, \mathbf{S}) \leq \epsilon$. Then,

$$
\mathscr{H}^{m}\left(E_{2}\right) \leq \int_{E_{2}} \# M_{x} d x \leq 2 \epsilon
$$


Proof. The Jacobian of a map on an $m$-rectifiable set is bounded by the $m$ th power of its Lipschitz constant. The area formula leads to,

$$
\begin{aligned}
\int_{E_{2}^{\prime}} \# M_{x} d x & \leq 2 \int_{E_{2}^{\prime}} \# M_{x}-1 d x \\
& =2 \int_{\pi_{V}^{-1}\left(E_{2}^{\prime}\right) \cap M_{T}} J\left(\pi_{V} \mid M_{T}\right)(z) d \mathscr{H}^{m}(z)-2 \mathscr{H}^{m}\left(E_{2}^{\prime}\right) \\
& \leq 2\left(\mathscr{H}^{m}\left(\pi_{V}{ }^{-1}\left(E_{2}^{\prime}\right) \cap M_{T}\right)-\mathscr{H}^{m}\left(E_{2}^{\prime}\right)\right) \\
& =2 \operatorname{Exc}\left(T, V, E_{2}^{\prime}, \mathbf{S}\right) \leq 2 \epsilon .
\end{aligned}
$$

A similar estimate is obtained by summing over the normed group elements in the preimage.

2.9. Lemma. Let $T \in \mathscr{R}_{m}(X ; G)$ and assume there is an m-plane $V \in \mathbf{G}(X, m)$ with $\operatorname{spt}(\partial T) \cap Z_{V}=\emptyset$. Let $g_{0} \in G$ be such that $\pi_{V \#}\left(T\left\llcorner Z_{V}\right)=g_{0} \llbracket \boldsymbol{B}_{V}(0,1) \rrbracket\right.$, then

$$
\sum_{y \in M_{x}}\|\mathbf{g}(y)\| \geq\left\|g_{0}\right\|
$$

for almost every $x \in \boldsymbol{B}_{V}(0,1)$. If further

$$
\max \left\{\operatorname{Exc}_{1}(T, V),\left\|g_{0}\right\| \operatorname{Exc}_{1}(T, V, \mathbf{S})\right\} \leq\left\|g_{0}\right\| \epsilon,
$$

then,

$$
\int_{E_{2}} \sum_{y \in M_{x}}\|\mathbf{g}(y)\| d x \leq 3\left\|g_{0}\right\| \epsilon
$$

Proof. The slices $\left\langle T, \pi_{V}, x\right\rangle$ are defined for almost every $x$ and concentrated on the finite set $M_{x}$, i.e. $\left\langle T, \pi_{V}, x\right\rangle=\sum_{y \in M_{x}} \pm \mathbf{g}(y) \llbracket y \rrbracket$ with + if $\pi_{V}: \operatorname{Tan}\left(M_{T}, y\right) \rightarrow V$ is orientation preserving and - otherwise. By [13, Theorem 3.8.1] we have

$$
\sum_{y \in M_{x}} \pm \mathbf{g}(y) \llbracket x \rrbracket=\pi_{V \#}\left\langle T, \pi_{V}, x\right\rangle=\left\langle\pi_{V \#} T, \pi_{V}, x\right\rangle=g_{0} \llbracket x \rrbracket
$$

for almost all $x \in \mathbf{B}_{V}(0,1)$ since on $\mathbf{B}_{V}(0,1)$ the chain $\pi_{V \#} T$ is a multiple of $g_{0}$. The first statement of the lemma now follows from the triangle inequality of the norm. By the assumption on the mass excess,

$$
\begin{aligned}
\int_{E_{2}^{\prime}} & \sum_{y \in M_{x}}\|\mathbf{g}(y)\|-\left\|g_{0}\right\| d x \\
& =\int_{\pi_{V}^{-1}\left(E_{2}^{\prime}\right) \cap M_{T}}\|\mathbf{g}(z)\| J\left(\pi_{V} \mid M_{T}\right)(z) d \mathscr{H}^{m}(z)-\left\|g_{0}\right\| \mathscr{H}^{m}\left(E_{2}^{\prime}\right) \\
& \leq \int_{\pi_{V}^{-1}\left(E_{2}^{\prime}\right) \cap M_{T}}\|\mathbf{g}(z)\| d \mathscr{H}^{m}(z)-\left\|g_{0}\right\| \mathscr{H}^{m}\left(E_{2}^{\prime}\right) \\
& =\operatorname{Exc}\left(T, V, E_{2}^{\prime}\right) \leq\left\|g_{0}\right\| \epsilon .
\end{aligned}
$$

By Lemma 2.8 it is also $\int_{E_{2}^{\prime}}\left\|g_{0}\right\| d x \leq 2\left\|g_{0}\right\| \epsilon$, hence the result follows.

Often we assume that we are away from the boundary and close to a plane, i.e. by truncating, translating and scaling we are in the situation where $\operatorname{spt}(\partial T) \cap Z_{V}=\emptyset$. By the constancy theorem for $G$ chains [14, Theorem 6.4] it follows that there is an 
element $g_{0} \in G$ such that $\pi_{V \#}\left(T\left\llcorner Z_{V}\right)=g_{0} \llbracket \mathbf{B}_{V}(0,1) \rrbracket\right.$. Next we give conditions such that the size excess is small if the mass excess is small.

2.10. Lemma. Let $T \in \mathscr{R}_{m}(X ; G), V \in \mathbf{G}(X, m)$ and $\epsilon>0$ such that

(1) $\operatorname{spt}(\partial T) \cap Z_{V}=\emptyset$,

(2) $\pi_{V \#}\left(T\left\llcorner Z_{V}\right)=g_{0} \llbracket \boldsymbol{B}_{V}(0,1) \rrbracket\right.$ for some $g_{0} \in G \backslash\left\{0_{G}\right\}$,

(3) $\Theta^{m}(\|T\|, x) \geq \frac{3}{4}\left\|g_{0}\right\|$ for $\|T\|$-almost every $x \in X$,

(4) $\operatorname{Exc}_{1}(T, V) \leq\left\|g_{0}\right\| \epsilon$.

Then $\operatorname{Exc}_{1}(T, V, \mathbf{S}) \leq 5 \epsilon$.

Proof. Let $M_{T} \subset Z_{V}$ be an $\mathscr{H}^{m}$-rectifiable set with $\|T\|\left(Z_{V} \backslash M_{T}\right)=0$ and $\mathbf{g}(x)=0_{G}$ for $x \notin M_{T}$. Since $\Theta^{m}(\|T\|, x)=\|\mathbf{g}(x)\|$ for $\|T\|$-almost every $x \in X$, assumption (3) is equivalent to $\|\mathbf{g}(x)\| \geq \frac{3}{4}\left\|g_{0}\right\|$ for $\|T\|$-almost every $x \in X$.

As before let $E_{2}$ be the set of those $x \in \mathbf{B}_{V}(0,1)$ for which $M_{x}:=\pi_{V}^{-1}\{x\} \cap M_{T}$ contains more than one point. For almost every $x \in \mathbf{B}_{V}(0,1) \backslash E_{2}$, the set $M_{x}$ consists of exactly one point $y$ for which $\|\mathbf{g}(y)\|=\left\|g_{0}\right\|$. Using the area formula and the first part of Lemma 2.9,

$$
\begin{aligned}
\left\|g_{0}\right\| \epsilon & \geq \int_{M_{T}}\|\mathbf{g}(x)\| d \mathscr{H}^{m}(x)-\left\|g_{0}\right\| \boldsymbol{\alpha}(m) \\
& \geq \int_{M_{T}}\|\mathbf{g}(x)\| J\left(\pi_{V} \mid M_{T}\right)(x) d \mathscr{H}^{m}(x)-\int_{\mathbf{B}_{V}(0,1)}\left\|g_{0}\right\| d x \\
& =\int_{\mathbf{B}_{V}(0,1)} \sum_{y \in M_{x}}\|\mathbf{g}(y)\|-\left\|g_{0}\right\| d x \\
& =\int_{E_{2}} \sum_{y \in M_{x}}\|\mathbf{g}(y)\|-\left\|g_{0}\right\| d x \\
& \geq \int_{E_{2}} \frac{6}{4}\left\|g_{0}\right\|-\left\|g_{0}\right\| d x \\
& =\frac{1}{2}\left\|g_{0}\right\| \mathscr{H}^{m}\left(E_{2}\right) .
\end{aligned}
$$

If $E \subset \mathbf{B}_{V}(0,1)$ is Borel measurable with $\mathscr{H}^{m}(E) \leq \delta$, then

$$
\begin{aligned}
\left\|g_{0}\right\| \mathscr{H}^{m}\left(M_{T} \cap \pi_{V}^{-1}(E)\right) & \leq \frac{4}{3} \mathbf{M}\left(T\left\llcorner\pi_{V}^{-1}(E)\right)\right. \\
& \leq \frac{4}{3}\left(\operatorname{Exc}(T, V, E)+\left\|g_{0}\right\| \mathscr{H}^{m}(E)\right) \\
& \leq \frac{4}{3}\left\|g_{0}\right\|(\epsilon+\delta) .
\end{aligned}
$$

Let $B_{2} \supset E_{2}$ be a Borel set with $\mathscr{L}^{m}\left(B_{2} \backslash E_{2}\right)=0$ and set $B_{1}:=\mathbf{B}_{V}(0,1) \backslash B_{2}$, . As shown above, it is $\mathscr{H}^{m}\left(B_{2}\right) \leq 2 \epsilon$ and hence

$$
\begin{aligned}
\operatorname{Exc}_{1}(T, V, \mathbf{S})= & \mathscr{H}^{m}\left(M_{T}\right)-\boldsymbol{\alpha}(m) \\
= & \mathscr{H}^{m}\left(M_{T} \cap \pi_{V}^{-1}\left(B_{1}\right)\right)-\mathscr{H}^{m}\left(B_{1}\right) \\
& +\mathscr{H}^{m}\left(M_{T} \cap \pi_{V}^{-1}\left(B_{2}\right)\right)-\mathscr{H}^{m}\left(B_{2}\right) \\
\leq & \left\|g_{0}\right\|^{-1} \operatorname{Exc}\left(T, V, B_{1}\right)+\mathscr{H}^{m}\left(M_{T} \cap \pi_{V}^{-1}\left(B_{2}\right)\right) \\
\leq & \epsilon+\frac{4}{3}(\epsilon+2 \epsilon) \leq 5 \epsilon .
\end{aligned}
$$


2.5. Quadratic forms associated to a chain. In order for a chain to be close to a submanifold near some point, it is necessary that it can locally be well approximated by planes. The quadratic form associated to a $G$-chain we introduce below will actually be used twice in these notes. First in the proof of the epiperimetric inequality in Section 3, and second, as part of the moments computations in Section 4.

For $T \in \mathscr{R}_{m}(X ; G), x \in X$ and $r>0$ we define the quadratic form

$$
Q(T, x, r)(y):=\frac{m+2}{\boldsymbol{\alpha}(m) r^{m+2}} \int_{\mathbf{B}(x, r)}\langle z-x, y\rangle^{2} d\|T\|(z) .
$$

The reason for this particular normalization is that in case $T=g \llbracket V \rrbracket$ for some oriented $m$-plane $V \in \mathbf{G}(X, m)$, then $Q(T, x, r)(y)=\|g\|\left|\pi_{V}(y)\right|^{2}$ for all $x \in V$ and $r>0$. This is demonstrated in Lemma 2.11 below. With $L_{Q}: X \rightarrow X$ we denote the self-adjoint operator associated to a quadratic form $Q$, i.e. $Q(y)=\left\langle y, L_{Q}(y)\right\rangle$. We now show that in case a chain is close to a plane $V$ near $x$, then $L_{Q(T, x, r)}$ is close to the orthogonal projection $\pi_{V}$.

2.11. Lemma. There is a constant $\mathbf{c}_{2.11}(m)>0$ with the following property. Let $T \in \mathscr{R}_{m}(X ; G)$ and $x \in \operatorname{spt}(T)$. Assume there is an $m$-plane $V \in \mathbf{G}(X, m)$ and $0<\rho<1,0<\epsilon<1$ with

(1) $\operatorname{spt}(\partial T) \cap Z_{V}(x, r)=\emptyset$,

(2) $\pi_{V \#}\left(T\left\llcorner Z_{V}(x, r)\right)=g_{0} \llbracket \boldsymbol{B}_{V}\left(\pi_{V}(x), r\right) \rrbracket\right.$ for some $g_{0} \in G \backslash\left\{0_{G}\right\}$,

(3) $\left|\pi_{V^{\perp}}(y-x)\right| \leq$ or for all $y \in \operatorname{spt}(T) \cap Z_{V}(x, r)$,

(4) $\Theta^{m}\left(\|T\|, x^{\prime}\right) \geq \frac{3}{4}\left\|g_{0}\right\|$ for $\|T\|$-a.e. $x^{\prime}$,

(5) $\operatorname{Exc}(T, V, x, r) \leq\left\|g_{0}\right\| \epsilon r^{m}$.

Then

$$
\begin{aligned}
\operatorname{tr}(Q(T, x, r))-m\left\|g_{0}\right\| & \leq \mathbf{c}_{2.11}\left\|g_{0}\right\|\left(\rho^{2}+\epsilon\right), \\
\left\|L_{Q(T, x, r)}-\right\| g_{0}\left\|\pi_{V}\right\| & \leq \mathbf{c}_{2.11}\left\|g_{0}\right\|(\rho+\epsilon) .
\end{aligned}
$$

Proof. By translating and rescaling $T$ we can assume that $x=0$ and $r=1$. This is justified by the scaling factor in the definition of $Q(T, x, r)$. Let $\left(e_{i}\right)_{i \geq 1}$ be an orthonormal basis of $X$ such that $e_{1}, \ldots, e_{m} \in V$. We define the quadratic form $Q^{\prime}$ on $X$ by

$$
Q^{\prime}(y):=\frac{m+2}{\boldsymbol{\alpha}(m)}\left\|g_{0}\right\| \int_{\mathbf{B}_{V}(0,1)}\langle y, z\rangle^{2} d z
$$


$Q^{\prime}$ is indeed a quadratic form with corresponding bilinear form $Q^{\prime}\left(y, y^{\prime}\right)=$ $\frac{m+2}{\alpha(m)}\left\|g_{0}\right\| \int_{\mathbf{B}_{V}(0,1)}\langle y, z\rangle\left\langle y^{\prime}, z\right\rangle d z$. If $y \in V$ with $|y|=1$, the symmetry of $Q^{\prime}$ implies that

$$
\begin{aligned}
\int_{\mathbf{B}_{V}(0, r)}\langle y, z\rangle^{2} d z & =\frac{1}{m} \sum_{i=1}^{m} \int_{\mathbf{B}_{V}(0, r)}\left\langle e_{i}, z\right\rangle^{2} d z \\
& =\frac{1}{m} \int_{\mathbf{B}_{V}(0, r)}|z|^{2} d z \\
& =\frac{1}{m} \int_{0}^{r} s^{2} \mathscr{H}^{m-1}\left(\mathbf{S}^{m-1}(0, s)\right) d s \\
& =\frac{1}{m} \int_{0}^{r} s^{m+1} \boldsymbol{\alpha}(m) m d r=\frac{\boldsymbol{\alpha}(m)}{m+2} r^{m+2} .
\end{aligned}
$$

Therefore, $Q^{\prime}(y)=\left\|g_{0}\right\|\left|\pi_{V}(y)\right|^{2}$ for all $y \in X$ and by the formula $2 Q^{\prime}\left(y, y^{\prime}\right)=$ $Q^{\prime}\left(y+y^{\prime}\right)-Q^{\prime}(y)-Q^{\prime}\left(y^{\prime}\right)$ we get

$$
Q^{\prime}\left(y, y^{\prime}\right)=\left\|g_{0}\right\|\left\langle\pi_{V}(y), \pi_{V}\left(y^{\prime}\right)\right\rangle=\left\langle y,\left\|g_{0}\right\| \pi_{V}\left(y^{\prime}\right)\right\rangle
$$

for all $y, y^{\prime} \in X$. Hence, $L_{Q^{\prime}}=\left\|g_{0}\right\| \pi_{V}$. We show next that $Q^{\prime}$ is close to $Q(T, 0,1)$.

By assumption $\operatorname{spt}\left(T\left\llcorner Z_{V}\left(\sqrt{1-\rho^{2}}\right)\right) \subset \operatorname{spt}(T\llcorner\mathbf{B}(0,1))\right.$ and hence the constancy theorem implies $\left(\pi_{V \#}(T\llcorner\mathbf{B}(0,1)))\left\llcorner\mathbf{B}_{V}\left(0, \sqrt{1-\rho^{2}}\right)=\right.\right.$ $g_{0} \llbracket \mathbf{B}_{V}\left(0, \sqrt{1-\rho^{2}}\right) \rrbracket$. Thus with the smallness of the excess,

$$
\left\|g_{0}\right\| \boldsymbol{\alpha}(m)\left(1-\rho^{2}\right)^{\frac{m}{2}} \leq \mathbf{M}\left(T\llcorner\mathbf{B}(0,1)) \leq\left\|g_{0}\right\|(\boldsymbol{\alpha}(m)+\epsilon) .\right.
$$

As before let $M_{T} \subset \operatorname{spt}(T) \cap Z_{V}$ be a $\sigma$-compact $\mathscr{H}^{m}$-rectifiable set on which $\| T\left\llcorner Z_{V} \|\right.$ is concentrated, and $\mathrm{g}: M_{T} \rightarrow G$ is a measurable function characterizing $T$. For $y \in \mathbf{B}(0,1)$ write the orthogonal decomposition with respect to $V$ as $y=$ $y_{v}+y_{v}^{\perp}$. The area formula leads to

$$
\begin{aligned}
& \int_{\mathbf{B}(0,1)}\langle y, z\rangle^{2} d\|T\|(z)=\int_{\mathbf{B}(0,1) \cap M_{T}}\|\mathbf{g}(z)\|\langle y, z\rangle^{2} d \mathscr{H}^{m}(z) \\
& \quad \geq \int_{\mathbf{B}(0,1) \cap M_{T}}\|\mathbf{g}(z)\|\langle y, z\rangle^{2} J\left(\pi_{V} \mid M_{T}\right)(z) d \mathscr{H}^{m}(z) \\
& \quad \geq \int_{\mathbf{B}_{V}\left(0, \sqrt{1-\rho^{2}}\right)} \sum_{z \in \pi_{V}^{-1}\left(z^{\prime}\right) \cap M_{T}}\|\mathbf{g}(z)\|\langle y, z\rangle^{2} d z^{\prime} \\
& \quad=\int_{\mathbf{B}_{V}\left(0, \sqrt{1-\rho^{2}}\right)} \sum_{z \in \pi_{V}^{-1}\left(z^{\prime}\right) \cap M_{T}}\|\mathbf{g}(z)\|\left(\left\langle y_{v}, z_{v}\right\rangle+\left\langle y_{v}^{\perp}, z_{v}^{\perp}\right\rangle\right)^{2} d z^{\prime} \\
& \geq \int_{\mathbf{B}_{V}\left(0, \sqrt{1-\rho^{2}}\right)}\left(\left\|g_{0}\right\|\left\langle y_{v}, z^{\prime}\right\rangle^{2}-2 \rho \sum_{z \in \pi_{V}^{-1}\left(z^{\prime}\right) \cap M_{T}}\|\mathbf{g}(z)\|\right) d z^{\prime} .
\end{aligned}
$$


Similarly,

$$
\begin{aligned}
\int_{\mathbf{B}_{V}(0,1)} \sum_{z \in \pi_{V}^{-1}\left(z^{\prime}\right) \cap M_{T}}\|\mathbf{g}(z)\| d z^{\prime} & =\int_{M_{T}}\|\mathbf{g}(z)\| J\left(\pi_{V} \mid M_{T}\right) d \mathscr{H}^{m}(z) \\
& \leq \int_{M_{T}}\|\mathbf{g}(z)\| d \mathscr{H}^{m}(z) \\
& =\mathbf{M}\left(T\left\llcorner Z_{V}\right)\right. \\
& \leq\left\|g_{0}\right\|(\epsilon+\boldsymbol{\alpha}(m)) .
\end{aligned}
$$

With (2.23),

$$
\begin{aligned}
Q(T, 0,1)(y) & =\frac{m+2}{\boldsymbol{\alpha}(m)} \int_{\mathbf{B}(0,1)}\langle y, z\rangle^{2} d\|T\|(z) \\
& \geq \frac{m+2}{\boldsymbol{\alpha}(m)}\left\|g_{0}\right\|\left[\int_{\mathbf{B}_{V}\left(0, \sqrt{1-\rho^{2}}\right)}\left\langle y_{v}, z^{\prime}\right\rangle^{2} d z^{\prime}-(\epsilon+\boldsymbol{\alpha}(m)) 2 \rho\right] \\
& =\left\|g_{0}\right\|\left(1-\rho^{2}\right)^{\frac{m+2}{2}}\left|y_{v}\right|^{2}-\frac{m+2}{\boldsymbol{\alpha}(m)}\left\|g_{0}\right\|(\epsilon+\boldsymbol{\alpha}(m)) 2 \rho \\
& \geq\left\|g_{0}\right\|\left|\pi_{V}(y)\right|^{2}-\mathbf{c}\left\|g_{0}\right\| \rho,
\end{aligned}
$$

for some $\mathbf{c}(m)>0$. To obtain an upper bound, a similar calculation shows,

$$
\begin{aligned}
\int_{\mathbf{B}(0,1)}\langle y, z\rangle^{2} d\|T\|(z) & \leq \int_{M_{T}}\|\mathbf{g}(z)\|\langle y, z\rangle^{2} d \mathscr{H}^{m}(z) \\
& =\int_{M_{T}}\|\mathbf{g}(z)\|\left(\left\langle y_{v}, z_{v}\right\rangle+\left\langle y_{v}^{\perp}, z_{v}^{\perp}\right\rangle\right)^{2} d \mathscr{H}^{m}(z) \\
& \leq \int_{M_{T}}\|\mathbf{g}(z)\|\left(\left\langle y_{v}, z_{v}\right\rangle^{2}+2 \rho+\rho^{2}\right) d \mathscr{H}^{m}(z) .
\end{aligned}
$$

Lemma 2.8 and Lemma 2.10 imply that $\mathscr{H}^{m}\left(E_{2}\right) \leq 10 \epsilon$ where $E_{2} \subset \mathbf{B}_{V}(0,1)$ is the set of those $x$ for which $M_{x}=\pi_{V}^{-1}\{x\} \cap M_{T}$ contains more than one point. By the first part of Lemma 2.9 we can find a Borel set $E_{1}$ such that $M_{x}=\left\{y_{x}\right\}$ and $\left\|\mathbf{g}\left(y_{x}\right)\right\|=\left\|g_{0}\right\|$ for $x \in E_{1}$ and $E_{1} \cup E_{2}$ has full measure in $\mathbf{B}_{V}(0,1)$. We can find countable many pairwise disjoint compact sets $K_{i} \subset \pi_{V}^{-1}\left(E_{1}\right) \cap M_{T}$ such that $\pi_{V}: K_{i} \rightarrow K_{i}^{\prime}:=\pi_{V}\left(K_{i}\right) \subset \mathbf{B}_{V}(0,1)$ is bi-Lipschitz with inverse $\varphi_{i}$ such that $\sup J \varphi_{i} \leq 1+\epsilon$ and the remaining set $M^{\prime}:=M_{T} \cap \pi_{V}{ }^{-1}\left(E_{1}\right) \backslash \cup_{i} K_{i}$ satisfies $\mathscr{H}^{m}\left(\pi_{V}\left(M^{\prime}\right)\right)=0$. By replacing $E_{1}$ with $\cup_{i} K_{i}^{\prime}$ if necessary, we can assume that $M^{\prime}=\emptyset$. The compact sets $K_{i}^{\prime}$ are pairwise disjoint since every $x \in E_{1}$ has only one preimage in $M_{T}$. Using the additivity of the excess,

$$
\begin{aligned}
\left\|g_{0}\right\| \epsilon \geq \operatorname{Exc}\left(T, V, E_{1}\right) & =\sum_{i} \int_{K_{i}}\|\mathbf{g}(z)\| d \mathscr{H}^{m}(z)-\int_{K_{i}^{\prime}}\left\|g_{0}\right\| d z^{\prime} \\
& =\left\|g_{0}\right\| \sum_{i} \int_{K_{i}^{\prime}}\left(J \varphi_{i}\left(z^{\prime}\right)-1\right) d z^{\prime} \\
& \geq 4^{-1}\left\|g_{0}\right\| \sum_{i} \int_{K_{i}^{\prime}}\left(\left\langle y_{v}, z^{\prime}\right\rangle^{2}+3 \rho\right)\left(J \varphi_{i}\left(z^{\prime}\right)-1\right) d z^{\prime} .
\end{aligned}
$$

Similarly,

$$
\mathbf{M}\left(T\left\llcorner\pi_{V}^{-1}\left(\mathbf{B}_{V}(0,1) \backslash E_{1}\right)\right) \leq\left\|g_{0}\right\|\left(\mathscr{H}^{m}\left(E_{2}\right)+\epsilon\right) \leq 11\left\|g_{0}\right\| \epsilon .\right.
$$


Combining these estimates with (2.24) leads to

$$
\begin{aligned}
\int_{\mathbf{B}(0,1)}\langle y, z\rangle^{2} d\|T\|(z) \leq & \int_{M_{T}}\|\mathbf{g}(z)\|\left(\left\langle y_{v}, z_{v}\right\rangle^{2}+2 \rho+\rho^{2}\right) d \mathscr{H}^{m}(z) \\
\leq & \int_{\pi_{V^{-1}}\left(E_{1}\right) \cap M_{T}}\|\mathbf{g}(z)\|\left(\left\langle y_{v}, z_{v}\right\rangle^{2}+3 \rho\right) d \mathscr{H}^{m}(z) \\
& +\mathbf{M}\left(T\left\llcorner\pi_{V}^{-1}\left(\mathbf{B}_{V}(0,1) \backslash E_{1}\right)\right)(1+3 \rho)\right. \\
\leq & \left\|g_{0}\right\| \sum_{i} \int_{K_{i}^{\prime}}\left(\left\langle y_{v}, z^{\prime}\right\rangle^{2}+3 \rho\right) J \varphi_{i}\left(z^{\prime}\right) d z^{\prime}+44\left\|g_{0}\right\| \epsilon \\
\leq & \left\|g_{0}\right\| \sum_{i} \int_{K_{i}^{\prime}}\left(\left\langle y_{v}, z^{\prime}\right\rangle^{2}+3 \rho\right) d z^{\prime}+48\left\|g_{0}\right\| \epsilon \\
\leq & \left\|g_{0}\right\| \int_{\mathbf{B}_{V}(0,1)}\left\langle y_{v}, z^{\prime}\right\rangle^{2} d z^{\prime}+3\left\|g_{0}\right\| \boldsymbol{\alpha}(m) \rho+48\left\|g_{0}\right\| \epsilon .
\end{aligned}
$$

Hence, $Q(T, 0,1)(y) \leq Q^{\prime}(y)+\mathbf{c}\left\|g_{0}\right\|(\rho+\epsilon)$ for some $\mathbf{c}(m)>0$. And with the lower bound we obtain $\left|Q(T, 0,1)(y)-Q^{\prime}(y)\right| \leq \mathbf{c}\left\|g_{0}\right\|(\rho+\epsilon)|y|^{2}$ for all $y \in X$. It follows directly that

$$
\left|\left\langle y^{\prime},\left(L_{Q(T, 0,1)}-L_{Q^{\prime}}\right)(y)\right\rangle\right|=\left|Q(T, 0,1)\left(y^{\prime}, y\right)-Q^{\prime}\left(y^{\prime}, y\right)\right| \leq \mathbf{c}\left\|g_{0}\right\|(\rho+\epsilon),
$$

for all $y, y^{\prime} \in \mathbf{B}(0,1)$. If we set $y^{\prime}=\left(L_{Q(T, 0,1)}-L_{Q^{\prime}}\right)(y) /\left|\left(L_{Q(T, 0,1)}-L_{Q^{\prime}}\right)(y)\right|$ we get the result about the projection.

Next we estimate the trace of the quadratic form. By the monotone convergence theorem,

$$
\begin{aligned}
\operatorname{tr} Q(T, 0,1) & =\sum_{i \geq 1} Q(T, 0,1)\left(e_{i}\right)=\frac{m+2}{\boldsymbol{\alpha}(m)} \int_{\mathbf{B}(0,1)} \sum_{i \geq 1}\left\langle z, e_{i}\right\rangle^{2} d\|T\|(z) \\
& =\frac{m+2}{\boldsymbol{\alpha}(m)} \int_{\mathbf{B}(0,1)}|z|^{2} d\|T\|(z) .
\end{aligned}
$$

With similar calculations as before,

$$
\begin{aligned}
\int_{\mathbf{B}(0,1)}|z|^{2} d\|T\|(z) \leq & \int_{M_{T}}\|\mathbf{g}(z)\|\left(\left|z_{v}\right|^{2}+\rho^{2}\right) d \mathscr{H}^{m}(z) \\
\leq & \int_{\pi_{V^{-1}}\left(E_{1}\right) \cap M_{T}}\|\mathbf{g}(z)\|\left(\left|z_{v}\right|^{2}+\rho^{2}\right) d \mathscr{H}^{m}(z) \\
& +\mathbf{M}\left(T\left\llcorner\pi_{V}^{-1}\left(\mathbf{B}_{V}(0,1) \backslash E_{1}\right)\right)\left(1+\rho^{2}\right)\right. \\
\leq & \left\|g_{0}\right\| \sum_{i} \int_{K_{i}^{\prime}}\left(\left|z^{\prime}\right|^{2}+\rho^{2}\right) J \varphi_{i}\left(z^{\prime}\right) d z^{\prime}+22\left\|g_{0}\right\| \epsilon \\
\leq & \left\|g_{0}\right\| \sum_{i} \int_{K_{i}^{\prime}}\left|z^{\prime}\right|^{2}+\rho^{2} d z^{\prime}+24\left\|g_{0}\right\| \epsilon \\
\leq & \left\|g_{0}\right\| \int_{\mathbf{B}_{V}(0,1)}\left|z^{\prime}\right|^{2} d z^{\prime}+\boldsymbol{\alpha}(m)\left\|g_{0}\right\| \rho^{2}+24\left\|g_{0}\right\| \epsilon .
\end{aligned}
$$

Since $\operatorname{tr} Q^{\prime}=m\left\|g_{0}\right\|$, the result follows.

It is easy to check that the quadratic forms $Q(T, x, r)$ are compact. Without loss of generality assume that $x=0$ and $r=1$. If $\left(y_{i}\right)$ is a sequence in $X$ converging weakly to $y$, then there is a finite constant $C>0$ such that $\left|y_{i}\right| \leq C$ for all $i$. For 
any $z \in \mathbf{B}(0,1)$ we get $\left\langle y_{i}, z\right\rangle^{2} \leq C^{2}$. So, by Lebesgue's Dominated Convergence Theorem and using the fact that $\|T\|$ is a finite measure,

$$
\begin{aligned}
\lim _{i \rightarrow \infty} Q(T, 0,1)\left(y_{i}\right) & =\lim _{i \rightarrow \infty} \frac{m+2}{\boldsymbol{\alpha}(m)} \int_{\mathbf{B}(0,1)}\left\langle y_{i}, z\right\rangle^{2} d\|T\|(z) \\
& =\frac{m+2}{\boldsymbol{\alpha}(m)} \int_{\mathbf{B}(0,1)} \lim _{i \rightarrow \infty}\left\langle y_{i}, z\right\rangle^{2} d\|T\|(z) \\
& =\frac{m+2}{\boldsymbol{\alpha}(m)} \int_{\mathbf{B}(0,1)}\langle y, z\rangle^{2} d\|T\|(z)=Q(T, x, r)(y) .
\end{aligned}
$$

It follows from the spectral theorem that there is an orthonormal basis $\left(e_{k}\right) \subset X$ of eigenvectors of $L_{Q(T, x, r)}$. The only accumulation point of the set of eigenvalues $\left(\lambda_{k}\right)$ corresponding to $\left(e_{k}\right)$ is 0 and we can assume that this sequence is ordered as $\lambda_{1} \geq \lambda_{2} \geq \cdots \geq 0$. Let $W_{x, r} \in \mathbf{G}(X, m)$ be the $m$-plane spanned by $e_{1}, \ldots, e_{m}$.

2.12. Lemma. Assume $T$ satisfies the same assumptions as in Lemma 2.11 for $x \in X, r>0$ and an m-plane $V \in \mathbf{G}(X, m)$. If $\mathbf{c}_{2.11}(\rho+\epsilon)<1$, then $\left|\lambda_{i}-\left\|g_{0}\right\|\right| \leq$ $\mathbf{c}_{2.11}\left\|g_{0}\right\|(\rho+\epsilon)$ for $i=1, \ldots, m$ and $W_{x, r}$ is close to $V$ in the sense that

$$
\left\|\pi_{W_{x, r}}-\pi_{V}\right\| \leq 2 \sqrt{m} \mathbf{c}_{2.11}(\rho+\epsilon) .
$$

Proof. Again we assume that $x=0$ and $r=1$. Let $v$ be a unit vector in $V$ orthogonal to $V^{\prime}:=\operatorname{span}\left\{e_{1}, \ldots, e_{m-1}\right\}$. Such a vector exists since orthogonal projection $\pi_{V^{\prime}}: V \rightarrow V^{\prime}$ has nontrivial kernel. We can write $v=\sum_{i \geq m} v_{i} e_{i}$ with $1=|v|^{2}=\sum_{i \geq m} v_{i}^{2}$. By Lemma 2.11 we get $\| g_{0}|| v|-| L_{Q(T, x, r)}(v) \mid \leq$ $\mathbf{c}_{2.11}\left\|g_{0}\right\|(\rho+\epsilon)|v|$ and further,

$$
\left\|g_{0}\right\|^{2}\left(1-\mathbf{c}_{2.11}(\rho+\epsilon)\right)^{2} \leq\left|L_{Q(T, x, r)}(v)\right|^{2}=\sum_{i \geq m}\left|\lambda_{i} v_{i}\right|^{2} \leq \lambda_{m}^{2} .
$$

Hence, $\left\|g_{0}\right\|\left(1-\mathbf{c}_{2.11}(\rho+\epsilon)\right) \leq \lambda_{m} \leq \cdots \leq \lambda_{1}$. To obtain the upper bound we use again Lemma 2.11,

$$
\begin{aligned}
\lambda_{1} & \leq\left|\left\|g_{0}\right\| \pi_{V}\left(e_{1}\right)-\lambda_{1} e_{1}\right|+\left\|g_{0}\right\|\left|\pi_{V}\left(e_{1}\right)\right| \\
& \leq\left|\left\|g_{0}\right\| \pi_{V}\left(e_{1}\right)-L_{Q(T, x, r)}\left(e_{1}\right)\right|+\left\|g_{0}\right\| \\
& \leq \mathbf{c}_{2.11}\left\|g_{0}\right\|(\rho+\epsilon)+\left\|g_{0}\right\| .
\end{aligned}
$$

This shows the first estimate of the lemma. For $i=1, \ldots, m$, this implies that

$$
\begin{aligned}
\left\|g_{0}\right\|\left|e_{i}-\pi_{V}\left(e_{i}\right)\right| & \leq\left|\left\|g_{0}\right\| e_{i}-\lambda_{i} e_{i}\right|+\left|L_{Q(T, x, r)}\left(e_{i}\right)-\left\|g_{0}\right\| \pi_{V}\left(e_{i}\right)\right| \\
& \leq 2 \mathbf{c}_{2.11}\left\|g_{0}\right\|(\rho+\epsilon),
\end{aligned}
$$

and therefore $\left|w-\pi_{V}(w)\right| \leq 2 \sqrt{m} c|w|$ for all $w \in W_{x, r}$. With Lemma 2.1, the proof is finished.

\section{REIFENBERG'S EPIPERIMETRIC INEQUALITY FOR A POLYHEDRAL CONE}

This section essentially contains the results covered by Reifenberg in [20]. In the setting of manifolds they can also be found in the book [17] of Morrey. The proofs here often contain a bit more detail, and where appropriate we use the tools for chains such as slicing and push-forwards to clarify the arguments. The main object in this section is a polyhedral cone $P \in \mathscr{P}_{m}(X ; G)$ in a Hilbert space $X$ with coefficients in a normed Abelian group $G$. We assume that $\operatorname{spt}(P)$ has finite diameter but we could as well work with the infinite cone generated by $P$. The main 
statement of this section is Theorem 3.18 which essentially states that if $P$ is close to an $m$-plane $V \in \mathbf{G}(X, m)$, then there is a comparison surface $S \in \mathscr{P}_{m}(X ; G)$ with $\partial(S\llcorner\mathbf{B}(0,1))=\partial(P\llcorner\mathbf{B}(0,1))$ and

$$
\mathbf{M}\left(S\llcorner\mathbf{B}(0,1))-\left\|g_{0}\right\| \boldsymbol{\alpha}(m) \leq \lambda\left(\mathbf{M}\left(P\llcorner\mathbf{B}(0,1))-\left\|g_{0}\right\| \boldsymbol{\alpha}(m)\right),\right.\right.
$$

for some constant $0<\lambda(m)<1$ and some $g_{0} \in G$ representing the group element of the projection from $P$ to $V$. We will apply this theorem in Proposition 5.4 to a rectifiable chain by approximating it with polyhedral chains which is justified by [10].

3.1. Assumptions and preliminaries. Until further notice we assume that $0 \leq$ $\rho<\frac{1}{2}, 0 \leq \epsilon<\frac{1}{2}$ and $V \in \mathbf{G}(X, m)$ is an $m$-plane with:

(1) $P$ is in general position with respect to $V$, i.e. $P$ can be written as a finite formal sum $\sum_{i} g_{i} \llbracket S_{i} \rrbracket$ of oriented $m$-simplices $S_{i}$ and $g_{i} \in G$ such that $\pi_{V}$ restricted to $S_{i}$ is one-to-one and orientation preserving.

(2) $\operatorname{spt}(\partial P) \cap Z_{V}(2)=\emptyset$.

(3) $P\left\llcorner Z_{V}\right.$ is 1-homogeneous in its domain, i.e. $\eta_{r \#}\left(P\left\llcorner Z_{V}\right)=P\left\llcorner Z_{V}(r)\right.\right.$ where $\eta_{r}(p)=r p$ is the scaling by $r \in[0,2]$ in $X$.

(4) $\pi_{V \#}\left(P\left\llcorner Z_{V}\right)=g_{0} \llbracket \mathbf{B}_{V}(0,1) \rrbracket\right.$ for some $g_{0} \in G \backslash\left\{0_{G}\right\}$.

(5) $\left\|g_{i}\right\| \geq \frac{3}{4}\left\|g_{0}\right\|$.

(6) $\operatorname{Exc}_{1}(P, V) \leq\left\|g_{0}\right\| \epsilon$.

(7) $\left|\pi_{V^{\perp}}(x)\right| \leq \rho$ for all $x \in \operatorname{spt}(P) \cap Z_{V}$.

(8) $\epsilon \leq \rho^{6 m}$.

Combining (4) and (6) we get

$$
\mathbf{M}\left(P\left\llcorner Z_{V}\right)-\left\|g_{0}\right\| \boldsymbol{\alpha}(m) \leq\left\|g_{0}\right\| \epsilon .\right.
$$

Similarly, it follows directly from Lemma 2.10 that

$$
\operatorname{Exc}_{1}(P, V, \mathbf{S})=\mathscr{H}^{m}\left(\operatorname{spt}(P) \cap Z_{V}\right)-\boldsymbol{\alpha}(m) \leq 5 \epsilon .
$$

For the most part of this section we could replace (5) by a bound like $\operatorname{Exc}_{1}(P, V, \mathbf{S}) \leq$ $\epsilon$. The properties for $P$ stated above are scaling invariant with respect to $\left\|g_{0}\right\|$ and by replacing the group norm $\|\cdot\|$ with $\|\cdot\| /\left\|g_{0}\right\|$ if necessary we may assume that $\left\|g_{0}\right\|=1$.

By the general position assumption (1) we can write $P=\sum_{i} g_{i} \llbracket S_{i} \rrbracket$ for finitely many almost disjoint oriented $m$-simplices $S_{i}$ and $g_{i} \neq 0_{G}$. Because $P$ is 1 homogeneous we can assume that $S_{i}$ is the convex hull of $S_{i}^{\prime} \cup\{0\}$ where $S_{i}^{\prime}$ is some $(m-1)$-simplex in $X \backslash Z_{V}(2)$. It follows that $\operatorname{spt}(P)=\bigcup_{i} S_{i}$ and therefore the set $P_{x}:=\pi_{V}^{-1}(x) \cap \operatorname{spt}(P)$ is finite for all $x \in \mathbf{B}_{V}(0,1)$. Any such simplex $S_{i}$ can be uniquely expressed as the graph of an affine map $\mathbf{y}^{i}: \pi_{V}\left(S_{i}\right) \rightarrow V^{\perp}$. Let $I_{x}$ be the collection of all $i$ for which $x \in \pi_{V}\left(S_{i}\right)$. Note that for almost every $x \in \mathbf{B}_{V}(0,1), x+\mathbf{y}^{i}(x)$ is in the interior of $S_{i}$ for every $i \in I_{x}$. Further, for almost every $x \in \mathbf{B}_{V}(0,1)$,

$$
\sum_{i \in I_{x}} g_{i}=g_{0}
$$

since we assume that $\pi_{V}: S_{i} \rightarrow V$ is orientation preserving. The map $\mathbf{y}^{i}$ is defined in a neighborhood of $x$ if $x+\mathbf{y}^{i}(x)$ is in the interior of $S_{i}$. As in Subsections 2.4 and 2.5 let $E_{1}$ be the points in $\mathbf{B}_{V}(0,1)$ where $\pi_{V}$ has only one preimage in $\operatorname{spt}(P)$ lying in the interior of some simplex $S_{i}$ and $E_{2}=\mathbf{B}_{V}(0,1) \backslash E_{1}$ be the complement. 
For a point $x \in E_{1}$ we write $\mathbf{y}(x)$ for the only element in $V^{\perp}$ with $x+\mathbf{y}(x) \in \operatorname{spt}(P)$. Some immediate consequences of Lemma 2.8, Lemma 2.9 and (3.1) are

$$
\begin{gathered}
\mathscr{H}^{m}\left(E_{2}\right) \leq \int_{E_{2}} \# I_{x} d x \leq 10 \epsilon, \\
\int_{E_{2}} \sum_{i \in I_{x}}\left\|g_{i}\right\| d x \leq 15\left\|g_{0}\right\| \epsilon .
\end{gathered}
$$

Further, by our assumptions on $P$ and the area formula, there holds for any Borel set $B \subset V$,

$$
\mathscr{H}^{m}\left(\operatorname{spt}(P) \cap \pi_{V}^{-1}(B)\right)=\sum_{i} \mathscr{H}^{m}\left(S_{i} \cap \pi_{V}^{-1}(B)\right)=\int_{B} \sum_{i \in I_{x}}\left(1+\left(\mathcal{J} \mathbf{y}_{x}^{i}\right)^{2}\right)^{\frac{1}{2}} d x,
$$

and

$$
\mathbf{M}\left(P\left\llcorner\pi_{V}^{-1}(B)\right)=\sum_{i} \mathbf{M}\left(g_{i} \llbracket S_{i} \rrbracket\left\llcorner\pi_{V}^{-1}(B)\right)=\int_{B} \sum_{i \in I_{x}}\left\|g_{i}\right\|\left(1+\left(\mathcal{J} \mathbf{y}_{x}^{i}\right)^{2}\right)^{\frac{1}{2}} d x .\right.\right.
$$

We know that $\left\langle P, \pi_{V}, x\right\rangle$ is in $\mathscr{P}_{0}(X ; G)$ for almost every $x \in V$ and because of the observation above we get that

$$
\left\langle P, \pi_{V}, x\right\rangle=\sum_{i \in I_{x}} g_{i} \llbracket x+\mathbf{y}^{i}(x) \rrbracket .
$$

As in [20] we often use orthogonal coordinates $x \in V$ and $y \in V^{\perp}$.

We define for any $x \in \mathbf{B}_{V}(0,1)$,

$$
\|D \mathbf{y}\|(x):=\sum_{i \in I_{x}}\left\|D \mathbf{y}_{x}^{i}\right\|
$$

and similarly $\|D \mathbf{y}\|_{\mathrm{HS}}(x)$ is defined.

Here are some basic estimates we will need later on.

3.1. Lemma ([20, Lemma 1]). Let $a, b \geq 0, a_{1}, \ldots, a_{k} \geq 0, \lambda_{1}, \ldots, \lambda_{k} \geq 0$ with $\lambda_{1}+\cdots+\lambda_{k}=1$ and $\delta \in(0,1]$. Then

$$
\begin{aligned}
\left(1+a^{2}+b^{2}\right)^{\frac{1}{2}} & \leq\left(1+a^{2}\right)^{\frac{1}{2}}+\left(\left(1+b^{2}\right)^{\frac{1}{2}}-1\right), \\
\left(1+\left(\lambda_{1} a_{1}+\cdots+\lambda_{k} a_{k}\right)^{2}\right)^{\frac{1}{2}} & \leq \lambda_{1}\left(1+a_{1}^{2}\right)^{\frac{1}{2}}+\cdots+\lambda_{k}\left(1+a_{k}^{2}\right)^{\frac{1}{2}}, \\
\left(1+\delta^{2} a^{2}\right)^{\frac{1}{2}}-1 & \leq \delta\left(\left(1+a^{2}\right)^{\frac{1}{2}}-1\right) \\
(1+a b)^{\frac{1}{2}}-1 & \leq \frac{1}{2} \delta^{-2} b^{2}+\delta\left(\left(1+a^{2}\right)^{\frac{1}{2}}-1\right) .
\end{aligned}
$$

Proof. Abbreviating $\alpha^{2}=1+a^{2}, \beta^{2}=1+b^{2}$ and squaring both sides of (3.4) we notice it is equivalent to $\alpha+\beta \leq 1+\alpha \beta$, which readily holds as $\alpha \geq 1$ and $\beta \geq 1$. Next, introducing $h(t)=\sqrt{1+t^{2}}, t \geq 0$, we notice (3.5) expresses the convexity of $h$. Letting $g(s)=s^{-1} \int_{0}^{s} h^{\prime}=s^{-1}(h(s)-h(0))$ we note (3.6) is equivalent to $g$ being nondecreasing. Computing $g^{\prime}$ this in turn is equivalent to $s^{-1} \int_{0}^{s} h^{\prime} \leq h^{\prime}(s)$, a consequence of the fact that $h^{\prime}$ is nondecreasing. In order to establish (3.7) we notice $h$ is nondecreasing, convex, $h(t) \leq 1+\frac{t^{2}}{2}$ and we apply (3.6) as follows : $h(\sqrt{a b})-1 \leq$ $h\left(\frac{1}{2} \frac{b}{\delta}+\frac{1}{2} \delta a\right)-1 \leq \frac{1}{2}\left(h\left(\frac{b}{\delta}\right)-1\right)+\frac{1}{2}(h(\delta a)-1) \leq \frac{1}{4} \frac{b^{2}}{\delta^{2}}+\frac{1}{2} \delta(h(\delta a)-1)$. 
Now, let $W$ be a $k$-dimensional subspace of $V$ and $W^{\perp}$ its orthogonal complement in $V$. Then for all $w^{\prime} \in W^{\perp}$ the slice $\left\langle P, \pi_{W^{\perp}}, w^{\prime}\right\rangle$ is defined and an element of $\mathscr{P}_{k}(X ; G)$. Writing $x=w+w^{\prime} \in W+W^{\perp}$ for some $x \in \mathbf{B}_{V}(0,1)$, we have by iterated slicing and the assumption on $P$,

$$
\left\langle\left\langle P, \pi_{W^{\perp}}, w^{\prime}\right\rangle, \pi_{W}, w\right\rangle=\left\langle P,\left(\pi_{W^{\perp}}, \pi_{W}\right),\left(w^{\prime}, w\right)\right\rangle=\left\langle P, \pi_{V}, x\right\rangle .
$$

So, for some fixed $w^{\prime} \in W^{\perp} \cap \mathbf{B}_{V}(0,1)$ the slice $P^{\prime}:=\left\langle P, \pi_{W^{\perp}}, w^{\prime}\right\rangle$ satisfies $\chi\left\langle P^{\prime}, \pi_{W}, w\right\rangle=\chi\left\langle P, \pi_{V}, x\right\rangle=g_{0}$ (where $\chi$ denotes the augmentation map) for all $w \in W$ with $w+w^{\prime} \in \mathbf{B}_{V}(0,1)$. Further,

$$
\partial P^{\prime}=(-1)^{m-k}\left\langle\partial P, \pi_{W^{\perp}}, w^{\prime}\right\rangle \text {, }
$$

and hence

$$
\operatorname{spt}\left(\partial P^{\prime}\right) \cap Z_{V}=\operatorname{spt}\left(\left\langle\partial P, \pi_{W^{\perp}}, w^{\prime}\right\rangle\right) \cap Z_{V} \subset \operatorname{spt}(\partial P) \cap Z_{V}=\emptyset .
$$

As a result $\operatorname{spt}\left(\partial\left(P^{\prime}\left\llcorner Z_{V}\right)\right) \subset \partial Z_{V}\right.$.

3.2. Mass estimate of the averaged cone. Let $\left[x_{1}, x_{2}\right]$ be the straight line segment connecting two different points $x_{1}, x_{2} \in \mathbf{B}_{V}(0,1)$. Let $W$ be the oriented $(m-1)$-dimensional subspace of $V$ orthogonal to $x_{2}-x_{1}$. By the general position assumption the set $\operatorname{spt}(P) \cap \pi_{V}^{-1}\left(\left[x_{1}, x_{2}\right]\right)$ is a union of finitely many straight lines each lying in some simplex $S_{i}$. We define the truncated slice,

$$
P\left(x_{1}, x_{2}\right):=\left\langle P, \pi_{W}, w\right\rangle\left\llcorner\pi_{V}^{-1}\left(\left[x_{1}, x_{2}\right]\right),\right.
$$

where $w \in W \cap \mathbf{B}_{V}(0,1)$ is the vector with $\pi_{W}\left(x_{2}\right)=\pi_{W}\left(x_{1}\right)=w$. By the observations above and some general facts about slices, $P\left(x_{1}, x_{2}\right) \in \mathscr{P}_{1}(X ; G)$, $\pi_{V \#} P\left(x_{1}, x_{2}\right)=g_{0} \llbracket x_{1}, x_{2} \rrbracket$ (given the right orientation on $W$ ) and hence $\pi_{V \#} \partial P\left(x_{1}, x_{2}\right)=g_{0} \llbracket x_{2} \rrbracket-g_{0} \llbracket x_{1} \rrbracket$.

3.2. Lemma ([20, Lemma 2]). If $x_{1}, x_{2} \in E_{1}$, then

$$
\left|\mathbf{y}\left(x_{1}\right)-\mathbf{y}\left(x_{2}\right)\right| \leq \int_{\left[x_{1}, x_{2}\right]}\|D \mathbf{y}\|(x) d \mathscr{H}^{1}(x) .
$$

Proof. As noted above, $P\left(x_{1}, x_{2}\right)$ is a well-defined non-zero polyhedral 1-chain with $\operatorname{support} \operatorname{in} \operatorname{spt}(P) \cap \pi_{V}^{-1}\left(\left[x_{1}, x_{2}\right]\right)$ and $\partial P\left(x_{1}, x_{2}\right)=g_{0} \llbracket x_{2}+\mathbf{y}\left(x_{2}\right) \rrbracket-g_{0} \llbracket x_{1}+\mathbf{y}\left(x_{1}\right) \rrbracket$. We want to show that there is a curve in $\operatorname{spt}\left(P\left(x_{1}, x_{2}\right)\right)$ connecting $x_{1}+\mathbf{y}\left(x_{1}\right)$ and $x_{2}+\mathbf{y}\left(x_{2}\right)$. As a polyhedral 1-chain, $P\left(x_{1}, x_{2}\right)$ is a finite sum of loops $g_{i} \llbracket L_{i} \rrbracket$ and curves $g_{j} \llbracket C_{j} \rrbracket$ connecting $x_{1}+\mathbf{y}\left(x_{1}\right)$ with $x_{2}+\mathbf{y}\left(x_{2}\right)$. For any loop, $\pi_{V \#}\left(g_{i} \llbracket L_{i} \rrbracket\right)=0$ by the constancy theorem, since $\partial\left(g_{i} \llbracket L_{i} \rrbracket\right)=0$. Since $\pi_{V \#} \sum_{j} g_{j} \llbracket C_{j} \rrbracket=g_{0} \llbracket x_{1}, x_{2} \rrbracket \neq$ 0 , the set of $C_{j}$ 's is not empty.

Thus there is a piecewise linear curve $\gamma:[0, L] \rightarrow \operatorname{spt}\left(P\left(x_{1}, x_{2}\right)\right)$ connecting $x_{1}+\mathbf{y}\left(x_{1}\right)$ and $x_{2}+\mathbf{y}\left(x_{2}\right)$ that is injective and satisfies $\left\|\gamma^{\prime}\right\|=1$ almost everywhere. By the area formula

$$
\int_{0}^{L} f(t)\left|\left(\pi_{V} \circ \gamma\right)^{\prime}(t)\right| d t=\int_{\left[x_{1}, x_{2}\right]} \sum_{t \in\left(\pi_{V} \circ \gamma\right)^{-1}(x)} f(t) d \mathscr{H}^{1}(x),
$$

for all measurable functions $f$. Set $f(t):=\left|\left(\pi_{V} \circ \gamma\right)^{\prime}(t)\right|^{-1}$. If $\gamma(t)$ is in some simplex $S_{i(t)}$ in a neighborhood of $t$, then by (2.6)

$$
f(t)=\left|\pi_{V}\left(\gamma^{\prime}(t)\right)\right|^{-1} \leq \operatorname{Lip}\left(\mathbf{y}^{i(t)}\right)=\left\|D \mathbf{y}^{i(t)}\right\| .
$$


Finally,

$$
\begin{aligned}
\left|\mathbf{y}\left(x_{1}\right)-\mathbf{y}\left(x_{2}\right)\right| \leq L & =\int_{0}^{L} f(t)\left|\left(\pi_{V} \circ \gamma\right)^{\prime}(t)\right| d t \\
& \leq \int_{\left[x_{1}, x_{2}\right]} \sum_{t \in\left(\pi_{V} \circ \gamma\right)^{-1}(x)}\left\|D \mathbf{y}_{x}^{i(t)}\right\| d \mathscr{H}^{1}(x) \\
& \leq \int_{\left[x_{1}, x_{2}\right]} \sum_{i \in I_{x}}\left\|D \mathbf{y}_{x}^{i}\right\| d \mathscr{H}^{1}(x),
\end{aligned}
$$

and by definition this is exactly the integral of $\|D \mathbf{y}\|$.

3.3. Lemma ([20, Lemma 3]). There is a constant $\mathbf{c}_{3.3}(m)>0$ with the following property. If $f$ is a measurable nonnegative homogeneous function of degree 0 on $V$ (i.e. $f(t x)=f(x)$ for all $t>0)$, then

$$
\int_{\boldsymbol{B}_{V}(0,1)} d x \int_{\boldsymbol{B}_{V}(x, \rho|x|)} d x^{\prime} \int_{\left[x, x^{\prime}\right]} d z \rho^{-m}|x|^{-m} f(z) \leq \mathbf{c}_{3.3} \rho \int_{\boldsymbol{B}_{V}(0,1)} f(x) d x .
$$

Proof. Let

$$
\begin{aligned}
Z & :=\{|x| \leq 1\} \times\left\{\left|x^{\prime}\right| \leq 1\right\} \times\{\lambda \in[0, \rho]\} \subset V^{2} \times \mathbb{R}, \\
Z^{\prime} & :=\left\{\left(x, x^{\prime}, x^{\prime \prime}\right) \in V^{3}:|x| \leq 1,\left|x-x^{\prime}\right| \leq \rho|x|, x^{\prime \prime} \in\left[x, x^{\prime}\right]\right\} \subset V^{3},
\end{aligned}
$$

and define $\varphi: Z \rightarrow Z^{\prime}$ by $\varphi\left(x, x^{\prime}, \lambda\right):=\left(x, x+\rho|x| x^{\prime}, x+\lambda|x| x^{\prime}\right)$ and $g: Z \rightarrow \mathbb{R}$ by $g\left(x, x^{\prime}, \lambda\right):=f\left(x+\lambda|x| x^{\prime}\right)$. $\varphi$ is bi-Lipschitz onto its image and $J \varphi_{\left(x, x^{\prime}, \lambda\right)}=$ $\rho^{m}|x|^{m+1}\left|x^{\prime}\right| \leq \rho^{m}|x|^{m}$ holds almost everywhere on $Z$. By the area formula,

$$
\begin{aligned}
\int_{|x| \leq 1} \int_{\left|x-x^{\prime}\right| \leq \rho|x|} \int_{z \in\left[x, x^{\prime}\right]} \rho^{-m|x|^{-m} f(z)} & \leq \int_{|x| \leq 1} \int_{\left|x^{\prime}\right| \leq 1} \int_{\lambda \in[0, \rho]} g\left(x, x^{\prime}, \lambda\right) \\
& =\int_{\left|x^{\prime}\right| \leq 1} \int_{\lambda \in[0, \rho]} \int_{|x| \leq 1} g\left(x, x^{\prime}, \lambda\right) \\
& \leq \boldsymbol{\alpha}(m) \rho \max _{\left|x^{\prime}\right| \leq 1, \lambda \in[0, \rho]} \int_{|x| \leq 1} f\left(x+\lambda|x| x^{\prime}\right) .
\end{aligned}
$$

By assumption $\lambda \leq \rho<\frac{1}{2}$. For fixed $\lambda$ and $x^{\prime}$, the map $\psi: x \mapsto x+\lambda|x| x^{\prime}$ is a bijection of $\mathbf{B}_{V}(0,1)$ onto a subset of $\mathbf{B}_{V}(0,2)$ with

$$
D \psi_{x}(v)=v+\frac{1}{|x|}\langle x, v\rangle \lambda x^{\prime}
$$

Hence $\operatorname{det} D \psi_{x}=1+\lambda \frac{1}{|x|}\left\langle x, x^{\prime}\right\rangle \in\left[\frac{1}{2}, \frac{3}{2}\right]$ since we assume that $\rho \leq \frac{1}{2}$. Therefore,

$$
\begin{aligned}
\int_{|x| \leq 1} f\left(x+\lambda|x| x^{\prime}\right) & =\int_{\mathbf{B}_{V}(0,1)} f(\psi(x)) d x \\
& =\int_{\psi\left(\mathbf{B}_{V}(0,1)\right)} f(x)\left(\operatorname{det} D \psi_{x}\right)^{-1} d x \\
& \leq 2 \int_{\mathbf{B}_{V}(0,2)} f(x) d x
\end{aligned}
$$

Finally,

$$
\int_{\mathbf{B}_{V}(0,2)} f(x) d x=\int_{\mathbf{B}_{V}(0,1)} 2^{m} f(2 x) d x=\int_{\mathbf{B}_{V}(0,1)} 2^{m} f(x) d x
$$


and the statement follows.

The next estimate and its proof is directly taken from [20].

3.4. Lemma ([20, Lemma 4]). Let $\mu$ be a finite measure on $E$ and $f \in L^{\infty}(E, \mu)$ a positive function. Then

$$
\min \left\{\left(\int_{E} f d \mu\right)^{2},\left(\int_{E} 1 d \mu\right)^{2}\right\} \leq 3\left(\int_{E} 1 d \mu\right)\left(\int_{E}\left(1+f^{2}\right)^{\frac{1}{2}}-1 d \mu\right) .
$$

Proof. The Cauchy-Schwarz inequality implies that

$$
\begin{aligned}
\left(\int_{E} f d \mu\right)^{2} & =\left(\int_{E} \frac{f}{\left(\left(1+f^{2}\right)^{\frac{1}{2}}+1\right)^{\frac{1}{2}}}\left(\left(1+f^{2}\right)^{\frac{1}{2}}+1\right)^{\frac{1}{2}} d \mu\right)^{2} \\
& \leq\left(\int_{E} \frac{f^{2}}{\left(1+f^{2}\right)^{\frac{1}{2}}+1} d \mu\right)\left(\int_{E}\left(1+f^{2}\right)^{\frac{1}{2}}+1 d \mu\right) \\
& =\left(\int_{E}\left(1+f^{2}\right)^{\frac{1}{2}}-1 d \mu\right)\left(\int_{E} 2+\left(\left(1+f^{2}\right)^{\frac{1}{2}}-1\right) d \mu\right) .
\end{aligned}
$$

If $\int_{E}\left(1+f^{2}\right)^{\frac{1}{2}}-1 d \mu \leq \int_{E} 1 d \mu$, it follows that

$$
\left(\int_{E} f d \mu\right)^{2} \leq\left(\int_{E}\left(1+f^{2}\right)^{\frac{1}{2}}-1 d \mu\right)\left(\int_{E} 3 d \mu\right),
$$

and otherwise,

$$
\left(\int_{E} 1 d \mu\right)^{2} \leq\left(\int_{E} 1 d \mu\right)\left(\int_{E}\left(1+f^{2}\right)^{\frac{1}{2}}-1 d \mu\right) .
$$

We define an average function $\overline{\mathbf{y}}: \mathbf{B}_{V}(0,2) \rightarrow V^{\perp}$ by

$$
\overline{\mathbf{y}}(x):=\sum_{i \in I_{x}} \delta_{i} \mathbf{y}^{i}(x):=\left(\sum_{i \in I_{x}}\left\|g_{i}\right\|\right)^{-1} \sum_{i \in I_{x}}\left\|g_{i}\right\| \mathbf{y}^{i}(x) .
$$

Note that for $\mathscr{H}^{m}$-a.e. $x \in \mathbf{B}_{V}(0,1)$ it is $\left\|g_{0}\right\| \leq \sum_{i \in I_{x}}\left\|g_{i}\right\|$. Obviously, $|\overline{\mathbf{y}}(x)| \leq \rho$ for $\mathscr{H}^{m}$-a.e. $x \in \mathbf{B}_{V}(0,1)$ and for $x \in E_{1}$ we have $\overline{\mathbf{y}}(x)=\mathbf{y}(x)$. A suitable smoothed version of $\mathbf{y}$ is obtained by the map $\mathbf{v}: \mathbf{B}_{V}(0,1) \rightarrow V^{\perp}$ with

$$
\mathbf{v}(x):=\left(\boldsymbol{\alpha}(m) \rho^{m}|x|^{m}\right)^{-1} \int_{\mathbf{B}_{V}(x, \rho|x|)} \overline{\mathbf{y}}(z) d z .
$$

Because $P$ is 1-homogeneous, so are $\overline{\mathbf{y}}$ and $\mathbf{v}$. Here are first estimates of $\mathbf{v}$.

3.5. Lemma ([20, Lemma 7$])$. For all $x \in \boldsymbol{B}_{V}(0,1)$,

$$
|\mathbf{v}(x)| \leq 2 \rho,
$$

and there is a constant $\mathbf{c}_{3.5}(\mathrm{~m})>0$ such that

$$
\int_{B_{V}(0,1)} \sum_{i \in I_{x}}\left|\mathbf{v}(x)-\mathbf{y}^{i}(x)\right|^{2} d x \leq \mathbf{c}_{3.5} \rho^{2} \epsilon .
$$


Proof. By the definition of $\overline{\mathbf{y}}$ and the assumption on the height bound of $P$ it is clear that $|\overline{\mathbf{y}}(x)| \leq \rho$ for $x \in \mathbf{B}_{V}(0,1)$. Fix some $x \in \mathbf{B}_{V}\left(0, \frac{1}{2}\right)$. To obtain shorter formulas we abbreviate $B_{x, \rho}:=\mathbf{B}_{V}(x, \rho|x|)$ and $\left|B_{x, \rho}\right|=\mathscr{H}^{m}\left(B_{x, \rho}\right)$. Clearly, $B_{x, \rho} \subset \mathbf{B}_{V}(0,1)$ and hence

$$
\begin{aligned}
|\mathbf{v}(x)| & \leq\left(\boldsymbol{\alpha}(m) \rho^{m}|x|^{m}\right)^{-1} \int_{\mathbf{B}_{V}(x, \rho|x|)}|\overline{\mathbf{y}}(z)| d z \\
& \leq\left|B_{x, \rho}\right|^{-1}\left|B_{x, \rho}\right| \rho=\rho .
\end{aligned}
$$

This immediately implies the first statement of the lemma. By (3.9) and (3.2) we have on $E_{2} \subset \mathbf{B}_{V}(0,1)$,

$$
\begin{aligned}
\int_{E_{2}} \sum_{i \in I_{x}}\left|\mathbf{v}(x)-\mathbf{y}^{i}(x)\right|^{2} d x & \leq \int_{E_{2}} \sum_{i \in I_{x}} 4 \max \left\{|\mathbf{v}(x)|,\left|\mathbf{y}^{i}(x)\right|\right\}^{2} d x . \\
& \leq 4 \rho^{2} \int_{E_{2}} \# I_{x} d x \\
& \leq 40 \epsilon \rho^{2}
\end{aligned}
$$

Using that $E_{2}$ is a cone, it follows with (3.2) and the bounds $\sqrt{\epsilon} \leq \rho^{m} \leq 1$ that

$$
\begin{aligned}
\left|B_{x, \rho}\right|^{-1} \mathscr{H}^{m}\left(E_{2} \cap \mathbf{B}_{V}(x, \rho|x|)\right) & \leq\left|B_{x, \rho}\right|^{-1} \mathscr{H}^{m}\left(E_{2} \cap \mathbf{B}_{V}(0,(1+\rho)|x|)\right) \\
& \leq\left|B_{x, \rho}\right|^{-1} 10 \epsilon(1+\rho)^{m}|x|^{m} \\
& \leq 10 \cdot 2^{m} \boldsymbol{\alpha}(m)^{-1} \sqrt{\epsilon}
\end{aligned}
$$

With (3.10) and (3.11) it follows that there is some $\mathbf{c}(m)>0$ with

$$
\begin{aligned}
& \int_{\mathbf{B}_{V}\left(0, \frac{1}{2}\right)} \sum_{i \in I_{x}}\left|\mathbf{v}(x)-\mathbf{y}^{i}(x)\right|^{2} d x \\
& \leq \mathbf{c} \epsilon \rho^{2}+\int_{E_{1} \cap \mathbf{B}_{V}\left(0, \frac{1}{2}\right)} \sum_{i \in I_{x}}\left[\left|B_{x, \rho}\right|^{-1} \int_{B_{x, \rho}} \overline{\mathbf{y}}\left(x^{\prime}\right) d x^{\prime}-\mathbf{y}^{i}(x)\right]^{2} d x \\
& \leq \mathbf{c} \epsilon \rho^{2}+\int_{E_{1} \cap \mathbf{B}_{V}\left(0, \frac{1}{2}\right)}\left[\left|B_{x, \rho}\right|^{-1} \int_{B_{x, \rho}}\left|\overline{\mathbf{y}}\left(x^{\prime}\right)-\mathbf{y}(x)\right| d x^{\prime}\right]^{2} d x \\
& \leq \mathbf{c} \epsilon \rho^{2}+\int_{E_{1} \cap \mathbf{B}_{V}\left(0, \frac{1}{2}\right)}\left[\left.|| B_{x, \rho}\right|^{-1} \int_{E_{1} \cap B_{x, \rho}}\left|\mathbf{y}\left(x^{\prime}\right)-\mathbf{y}(x)\right| d x^{\prime} \mid+\mathbf{c} \sqrt{\epsilon} \rho\right]^{2} d x .
\end{aligned}
$$

Because $(a+b)^{2} \leq 2\left(a^{2}+b^{2}\right)$ we get that the expression above is bounded by

$$
\mathbf{c}^{\prime} \epsilon \rho^{2}+2 \int_{E_{1} \cap \mathbf{B}_{V}\left(0, \frac{1}{2}\right)}\left[\left|B_{x, \rho}\right|^{-1} \int_{E_{1} \cap B_{x, \rho}}\left|\mathbf{y}\left(x^{\prime}\right)-\mathbf{y}(x)\right| d x^{\prime}\right]^{2} d x,
$$

for some $\mathbf{c}^{\prime}(m)>0$. Since $\left|x^{\prime}\right| \leq 2|x| \leq 1$ for $x^{\prime} \in B_{x, \rho}$ and $\mathbf{y}$ is 1-homogeneous,

$$
\int_{E_{1} \cap B_{x, \rho}}\left|\mathbf{y}\left(x^{\prime}\right)-\mathbf{y}(x)\right| d x^{\prime} \leq \int_{B_{x, \rho}} 3 \rho|x| d x^{\prime} \leq 3\left(1+\frac{1}{m}\right) \int_{B_{x, \rho}} d x^{\prime} \int_{\left[x, x^{\prime}\right]} 1 .
$$

By Lemma 3.2,

$$
\int_{E_{1} \cap B_{x, \rho}}\left|\mathbf{y}\left(x^{\prime}\right)-\mathbf{y}(x)\right| d x^{\prime} \leq \int_{B_{x, \rho}} d x^{\prime} \int_{\left[x, x^{\prime}\right]}\|D \mathbf{y}\|,
$$


and hence the second term of (3.12) is bounded by

$$
\mathbf{c}(m) \int_{E_{1}}\left[\left|B_{x, \rho}\right|^{-1} \min \left\{\int_{B_{x, \rho}} d x^{\prime} \int_{\left[x, x^{\prime}\right]}\|D \mathbf{y}\|, \int_{B_{x, \rho}} d x^{\prime} \int_{\left[x, x^{\prime}\right]} 1\right\}\right]^{2} d x .
$$

Hence we can apply Lemma 3.4 and (3.13) is smaller or equal

$$
\begin{aligned}
& 3 \mathbf{c}(m) \int_{E_{1}}\left|B_{x, \rho}\right|^{-2}\left(\int_{B_{x, \rho}} d x^{\prime} \int_{\left[x, x^{\prime}\right]} 1\right)\left(\int_{B_{x, \rho}} d x^{\prime} \int_{\left[x, x^{\prime}\right]}\left(1+\|D \mathbf{y}\|^{2}\right)^{\frac{1}{2}}-1\right) \\
& \quad \leq 3 \mathbf{c}(m) \int_{E_{1}}\left|B_{x, \rho}\right|^{-2}\left|B_{x, \rho}\right| \rho|x|\left(\int_{B_{x, \rho}} d x^{\prime} \int_{\left[x, x^{\prime}\right]}\left(1+\|D \mathbf{y}\|^{2}\right)^{\frac{1}{2}}-1\right) \\
& \quad \leq 3 \mathbf{c}(m) \int_{\mathbf{B}_{V}(0,1)}\left|B_{x, \rho}\right|^{-1} \rho|x| \int_{B_{x, \rho}} d x^{\prime} \int_{\left[x, x^{\prime}\right]}\left(1+\|D \mathbf{y}\|^{2}\right)^{\frac{1}{2}}-1 .
\end{aligned}
$$

Because $\|D \mathbf{y}\|$ is 0 -homogeneous, so is $\left(1+\|D \mathbf{y}\|^{2}\right)^{\frac{1}{2}}-1$ and by Lemma 3.3 the term above is smaller than or equal to

$$
3 \mathbf{c}(m) \boldsymbol{\alpha}(m)^{-1} \mathbf{c}_{3.3} \rho^{2} \int_{\mathbf{B}_{V}(0,1)}\left(1+\|D \mathbf{y}\|^{2}\right)^{\frac{1}{2}}-1 .
$$

Using $\left(1+\left(\sum_{i} a_{i}\right)^{2}\right)^{\frac{1}{2}} \leq \sum_{i}\left(1+a_{i}^{2}\right)^{\frac{1}{2}},\left\|D \mathbf{y}_{x}^{i}\right\| \leq \mathcal{J} \mathbf{y}_{x}^{i}$ for almost all $x$ and (3.1) we see that

$$
\begin{aligned}
\int_{\mathbf{B}_{V}(0,1)}\left(1+\|D \mathbf{y}\|^{2}\right)^{\frac{1}{2}}-1 & =\int_{\mathbf{B}_{V}(0,1)}\left(1+\left(\sum_{i \in I_{x}}\left\|D \mathbf{y}_{x}^{i}\right\|\right)^{2}\right)^{\frac{1}{2}}-1 d x \\
& \leq \int_{\mathbf{B}_{V}(0,1)} \sum_{i \in I_{x}}\left(1+\left\|D \mathbf{y}_{x}^{i}\right\|^{2}\right)^{\frac{1}{2}}-1 d x \\
& \leq \int_{\mathbf{B}_{V}(0,1)} \sum_{i \in I_{x}}\left(1+\left(\mathcal{J} \mathbf{y}_{x}^{i}\right)^{2}\right)^{\frac{1}{2}}-1 d x \\
& =\mathscr{H}^{m}\left(\operatorname{spt}(P) \cap Z_{V}\right)-\boldsymbol{\alpha}(m) \leq 5 \epsilon .
\end{aligned}
$$

Plugging this back into (3.12) gives that $\int_{\mathbf{B}_{V}\left(0, \frac{1}{2}\right)} \sum_{i \in I_{x}}\left|\mathbf{v}(x)-\mathbf{y}^{i}(x)\right|^{2} d x \leq \mathbf{c}^{\prime \prime} \rho^{2} \epsilon$ for some $\mathbf{c}^{\prime \prime}(m)>0$. By homogeneity the lemma follows.

Next we want to estimate the differential $D \mathbf{v}_{x}$. It will also become clear from the following proof that $\mathbf{v}$ is differentiable at all $x \neq 0$.

3.6. Lemma ([20, Lemma 8]). There is a constant $\mathbf{c}_{3.6}(m)>0$ such that for all $x \in \boldsymbol{B}_{V}(0,1) \backslash\{0\}$,

$$
\left\|D \mathbf{v}_{x}\right\|_{\mathrm{HS}} \leq\left(1+\mathbf{c}_{3.6} \rho\right)\left(\boldsymbol{\alpha}(m) \rho^{m}|x|^{m}\right)^{-1} \int_{\boldsymbol{B}_{V}(x, \rho|x|)}\left\|D \overline{\mathbf{y}}_{z}\right\|_{\mathrm{HS}} d z .
$$

Proof. By definition

$$
\mathbf{v}(x)=\left(\boldsymbol{\alpha}(m) \rho^{m}|x|^{m}\right)^{-1} \int_{\mathbf{B}_{V}(x, \rho|x|)} \overline{\mathbf{y}}(z) d z=\boldsymbol{\alpha}(m)^{-1} \int_{\mathbf{B}_{V}(0,1)} \overline{\mathbf{y}}(x+z \rho|x|) d z .
$$

The map $\overline{\mathbf{y}}$ is piecewise linear and in particular differentiable outside a set of measure zero. Let $v \in B_{V}(0,1)$ and $t \geq 0$. The Lebesgue Dominated Convergence 
Theorem implies that

$$
\begin{aligned}
& \frac{1}{t}(\mathbf{v}(x+t v)-\mathbf{v}(x)) \\
& \quad=\boldsymbol{\alpha}(m)^{-1} \int_{\mathbf{B}_{V}(0,1)} \frac{1}{t}(\overline{\mathbf{y}}(x+t v+z \rho|x+t v|)-\overline{\mathbf{y}}(x+z \rho|x|)) d z \\
& \quad \rightarrow \boldsymbol{\alpha}(m)^{-1} \int_{\mathbf{B}_{V}(0,1)} D \overline{\mathbf{y}}_{x+z \rho|x|}(v+z \rho\langle x, v\rangle) d z
\end{aligned}
$$

for $t \rightarrow 0$. Hence with (2.6),

$$
\left|D \mathbf{v}_{x}(v)\right| \leq \boldsymbol{\alpha}(m)^{-1} \int_{\mathbf{B}_{V}(0,1)}\left|D \overline{\mathbf{y}}_{x+z \rho|x|}(v)\right|+\rho\left\|D \overline{\mathbf{y}}_{x+z \rho|x|}\right\|_{\mathrm{HS}} d z .
$$

For an orthonormal basis $\left(e_{1}, \ldots, e_{m}\right)$ of $V$, using the multivariate Jensen inequality

$$
\left(\left(f a_{1}\right)^{2}+\cdots+\left(f a_{m}\right)^{2}\right)^{\frac{1}{2}} \leq f\left(a_{1}^{2}+\cdots+a_{m}^{2}\right)^{\frac{1}{2}},
$$

to $(3.15)$ leads to

$$
\begin{aligned}
\left\|D \mathbf{v}_{x}\right\|_{\mathrm{HS}} & =\left(\sum_{i}\left|D \mathbf{v}_{x}\left(e_{i}\right)\right|^{2}\right)^{\frac{1}{2}} \\
& \leq \boldsymbol{\alpha}(m)^{-1} \int_{\mathbf{B}_{V}(0,1)}\left(\sum_{i}\left(\left|D \overline{\mathbf{y}}_{x+z \rho|x|}\left(e_{i}\right)\right|+\rho\left\|D \overline{\mathbf{y}}_{x+z \rho|x|}\right\|_{\mathrm{HS}}\right)^{2}\right)^{\frac{1}{2}} d z \\
& \leq \boldsymbol{\alpha}(m)^{-1} \int_{\mathbf{B}_{V}(0,1)}\left(\left\|D \overline{\mathbf{y}}_{x+z \rho|x|}\right\|_{\mathrm{HS}}^{2}+\left(m \rho^{2}+2 \sqrt{m} \rho\right)\left\|D \overline{\mathbf{y}}_{x+z \rho|x|}\right\|_{\mathrm{HS}}^{2}\right)^{\frac{1}{2}} d z \\
& \leq(1+\sqrt{m} \rho) \boldsymbol{\alpha}(m)^{-1} \int_{\mathbf{B}_{V}(0,1)}\left\|D \overline{\mathbf{y}}_{x+z \rho|x|}\right\|_{\mathrm{HS}} d z \\
& \leq(1+\sqrt{m} \rho)\left(\boldsymbol{\alpha}(m) \rho^{m}|x|^{m}\right)^{-1} \int_{\mathbf{B}_{V}(x, \rho|x|)}\left\|D \overline{\mathbf{y}}_{z}\right\|_{\mathrm{HS}} d z .
\end{aligned}
$$

In the next Lemma we estimate the derivatives of $\mathbf{v}$ pointwise.

3.7. Lemma ([20, Lemma 9]). There is a constant $\mathbf{c}_{3.7}(m)>0$ such that $\left\|D \mathbf{v}_{x}\right\|_{\mathrm{HS}} \leq \mathbf{c}_{3.7} \epsilon^{\frac{1}{3}}$ and hence $|\mathbf{v}(x)| \leq \mathbf{c}_{3.7} \epsilon^{\frac{1}{3}}|x|$ for all $x \in \boldsymbol{B}_{V}(0,1)$.

Proof. Fix a point $x \in \mathbf{B}_{V}(0,1)$. For shorter formulas we abbreviate $B_{x, \rho}:=$ $\mathbf{B}_{V}(x, \rho|x|)$. The Cauchy-Schwarz inequality implies

$$
\begin{aligned}
& \int_{B_{x, \rho}}\|D \overline{\mathbf{y}}\|_{\mathrm{HS}}=\int_{B_{x, \rho}}\left(\|D \overline{\mathbf{y}}\|_{\mathrm{HS}}^{2}\right)^{\frac{1}{2}} \\
& =\int_{B_{x, \rho}}\left(\left(1+\|D \overline{\mathbf{y}}\|_{\mathrm{HS}}^{2}\right)^{\frac{1}{2}}-1\right)^{\frac{1}{2}}\left(\left(1+\|D \overline{\mathbf{y}}\|_{\mathrm{HS}}^{2}\right)^{\frac{1}{2}}+1\right)^{\frac{1}{2}} \\
& \leq\left(\int_{B_{x, \rho}}\left(1+\|D \overline{\mathbf{y}}\|_{\mathrm{HS}}^{2}\right)^{\frac{1}{2}}-1\right)^{\frac{1}{2}}\left(\int_{B_{x, \rho}}\left(1+\|D \overline{\mathbf{y}}\|_{\mathrm{HS}}^{2}\right)^{\frac{1}{2}}+1\right)^{\frac{1}{2}} \\
& =\left(\int_{B_{x, \rho}}\left(1+\|D \overline{\mathbf{y}}\|_{\mathrm{HS}}^{2}\right)^{\frac{1}{2}}-1\right)^{\frac{1}{2}}\left(2\left|B_{x, \rho}\right|+\int_{B_{x, \rho}}\left(1+\|D \overline{\mathbf{y}}\|_{\mathrm{HS}}^{2}\right)^{\frac{1}{2}}-1\right)^{\frac{1}{2}} .
\end{aligned}
$$


By definition $\overline{\mathbf{y}}(z)=\sum_{i \in I_{z}} \delta_{i}(z) \mathbf{y}^{i}(z)$, where $\delta_{i}(z)=\left\|g_{i}\right\|\left(\sum_{j \in I_{x}}\left\|g_{j}\right\|\right)^{-1} \leq$ $\left\|g_{i}\right\|\left\|g_{0}\right\|^{-1}$. For almost every $\xi \in \mathbf{B}_{V}(0,1)$ there holds,

$$
\left\|D \overline{\mathbf{y}}_{\xi}\right\|_{\mathrm{HS}}=\left\|D\left(\sum_{i \in I_{\xi}} \delta_{i} \mathbf{y}^{i}\right)_{\xi}\right\|_{\mathrm{HS}}=\left\|\sum_{i \in I_{\xi}} \delta_{i}(\xi) D \mathbf{y}_{\xi}^{i}\right\|_{\mathrm{HS}} \leq \sum_{i \in I_{\xi}} \delta_{i}(\xi)\left\|D \mathbf{y}_{\xi}^{i}\right\|_{\mathrm{HS}} .
$$

Using Lemma 3.1 and the fact that $\left\|D \mathbf{y}_{z}^{i}\right\|_{\mathrm{HS}} \leq \mathcal{J} \mathbf{y}_{z}^{i}$ for almost all $z$,

$$
\begin{aligned}
\int_{B_{x, \rho}}\left(1+\|D \overline{\mathbf{y}}\|_{\mathrm{HS}}^{2}\right)^{\frac{1}{2}}-1 & \leq \int_{B_{x, \rho}}\left(1+\left(\sum_{i \in I_{z}} \delta_{i}(z)\left\|D \mathbf{y}_{z}^{i}\right\|_{\mathrm{HS}}\right)^{2}\right)^{\frac{1}{2}}-1 d z \\
& \leq \frac{1}{\left\|g_{0}\right\|} \int_{B_{x, \rho}} \sum_{i \in I_{z}}\left\|g_{i}\right\|\left(1+\left\|D \mathbf{y}_{z}^{i}\right\|_{\mathrm{HS}}^{2}\right)^{\frac{1}{2}}-\left\|g_{0}\right\| d z \\
& \leq \frac{1}{\left\|g_{0}\right\|} \int_{\mathbf{B}_{V}(0,(1+\rho)|x|)} \sum_{i \in I_{z}}\left\|g_{i}\right\|\left(1+\left(\mathcal{J} \mathbf{y}_{z}^{i}\right)^{2}\right)^{\frac{1}{2}}-\left\|g_{0}\right\| d z \\
& =(1+\rho)^{m}|x|^{m}\left\|g_{0}\right\|^{-1} \operatorname{Exc}_{1}(P, V) \\
& \leq 2^{m}|x|^{m} \epsilon .
\end{aligned}
$$

Hence

$$
\begin{aligned}
\int_{B_{x, \rho}}\|D \overline{\mathbf{y}}\|_{\mathrm{HS}} & \leq\left(2^{m}|x|^{m} \epsilon\right)^{\frac{1}{2}}\left(2^{m}|x|^{m} \epsilon+2\left|B_{x, \rho}\right|\right)^{\frac{1}{2}} \\
& =\left(2^{m}|x|^{m} \epsilon\right)^{\frac{1}{2}}\left(2^{m}|x|^{m} \epsilon+2 \boldsymbol{\alpha}(m) \rho^{m}|x|^{m}\right)^{\frac{1}{2}} \\
& \leq \mathbf{c}(m)|x|^{m} \epsilon^{\frac{1}{2}},
\end{aligned}
$$

for some constant $\mathbf{c}(m)>0$. By Lemma 3.6 and because $\epsilon^{\frac{1}{6}} \leq \rho^{m}$ we get for $x \neq 0$,

$$
\begin{aligned}
\left\|D \mathbf{v}_{x}\right\|_{\mathrm{HS}} & \leq\left(1+\mathbf{c}_{3.6} \rho\right)\left(\boldsymbol{\alpha}(m) \rho^{m}|x|^{m}\right)^{-1} \mathbf{c}|x|^{m} \epsilon^{\frac{1}{2}} \\
& =\mathbf{c}\left(1+\mathbf{c}_{3.6} \rho\right) \boldsymbol{\alpha}(m)^{-1} \epsilon^{-\frac{1}{6}} \epsilon^{\frac{1}{2}} \\
& =\mathbf{c}^{\prime} \epsilon^{\frac{1}{3}}
\end{aligned}
$$

for some constant $\mathbf{c}^{\prime}(m)>0$.

Because $\left\|D \mathbf{v}_{x}\right\| \leq\left\|D \mathbf{v}_{x}\right\|_{\mathrm{HS}} \leq \sqrt{m}\left\|D \mathbf{v}_{x}\right\|$ the statement in the lemma above is true for both norms. Further estimates on the integral of this differential is needed. In the following lemma the particular definition of the averaging function $\overline{\mathbf{y}}(x)=\left(\sum_{j \in I_{x}}\left\|g_{j}\right\|\right)^{-1} \sum_{i \in I_{x}}\left\|g_{i}\right\| \mathbf{y}^{i}(x)$ is used in an essential way.

3.8. Lemma ([20, Lemma 10]). There is a constant $\mathbf{c}_{3.8}(m)>0$ such that

$$
\int_{B_{V}} \sum_{i \in I_{x}}\left\|g_{i}\right\|\left(1+\left\|D \mathbf{v}_{x}\right\|_{\mathrm{HS}}^{2}\right)^{\frac{1}{2}} d x \leq \mathbf{c}_{3.8}\left\|g_{0}\right\| \rho \epsilon+\int_{B_{V}} \sum_{i \in I_{x}}\left\|g_{i}\right\|\left(1+\left\|D \mathbf{y}_{x}^{i}\right\|_{\mathrm{HS}}^{2}\right)^{\frac{1}{2}} d x .
$$

Proof. For $x \in \mathbf{B}_{V}$ abbreviate again $B_{x, \rho}:=\mathbf{B}_{V}(x, \rho|x|)$. Combining (3.16) and (3.17) as obtained in the proof of Lemma 3.7 above with the estimate $\epsilon \leq \rho^{m+1}$, 
we get,

$$
\begin{aligned}
& \left(\int_{B_{x, \rho}}\|D \overline{\mathbf{y}}\|_{\mathrm{HS}}\right)^{2} \\
& \quad \leq\left(\int_{B_{x, \rho}}\left(1+\|D \overline{\mathbf{y}}\|_{\mathrm{HS}}^{2}\right)^{\frac{1}{2}}-1\right)\left(2\left|B_{x, \rho}\right|+\int_{B_{x, \rho}}\left(1+\|D \overline{\mathbf{y}}\|_{\mathrm{HS}}^{2}\right)^{\frac{1}{2}}-1\right) \\
& \quad \leq\left(\int_{B_{x, \rho}}\left(1+\|D \overline{\mathbf{y}}\|_{\mathrm{HS}}^{2}\right)^{\frac{1}{2}}-1\right)\left(2 \boldsymbol{\alpha}(m) \rho^{m}|x|^{m}+2^{m}|x|^{m} \epsilon\right) \\
& \quad \leq 2 \boldsymbol{\alpha}(m) \rho^{m}|x|^{m}(1+\mathbf{c} \rho) \int_{B_{x, \rho}}\left(1+\|D \overline{\mathbf{y}}\|_{\mathrm{HS}}^{2}\right)^{\frac{1}{2}}-1,
\end{aligned}
$$

for some constant $\mathbf{c}(m)>0$. Using the fact that $\left(1+a^{2}\right)^{\frac{1}{2}} \leq 1+\frac{1}{2} a^{2}$, it follows from Lemma 3.6 and (3.18) that for $\mathbf{c}^{\prime}=\max \left\{\mathbf{c}_{3.6}, \mathbf{c}\right\}$,

$$
\begin{aligned}
\int_{\mathbf{B}_{V}}(1+ & \left.\|D \mathbf{v}\|_{\mathrm{HS}}^{2}\right)^{\frac{1}{2}}-1 \leq \frac{1}{2} \int_{\mathbf{B}_{V}}\|D \mathbf{v}\|_{\mathrm{HS}}^{2} \\
& \leq \frac{1}{2} \int_{\mathbf{B}_{V}}\left[\left(1+\mathbf{c}_{3.6} \rho\right)\left(\boldsymbol{\alpha}(m) \rho^{m}|x|^{m}\right)^{-1} \int_{B_{x, \rho}}\|D \overline{\mathbf{y}}\|_{\mathrm{HS}}\right]^{2} \\
& \leq \frac{1}{2} \int_{\mathbf{B}_{V}} 2\left(1+\mathbf{c}^{\prime} \rho\right)^{3}\left(\boldsymbol{\alpha}(m) \rho^{m}|x|^{m}\right)^{-1} \int_{B_{x, \rho}}\left(1+\|D \overline{\mathbf{y}}\|_{\mathrm{HS}}^{2}\right)^{\frac{1}{2}}-1 \\
& =\int_{\mathbf{B}_{V}}\left(1+\mathbf{c}^{\prime} \rho\right)^{3} \boldsymbol{\alpha}(m)^{-1} \int_{\mathbf{B}_{V}}\left(1+\left\|D \overline{\mathbf{y}}_{x+\rho|x| z}\right\|_{\mathrm{HS}}^{2}\right)^{\frac{1}{2}}-1 d z \\
& \leq\left(1+\mathbf{c}^{\prime} \rho\right)^{3} \max _{z \in \mathbf{B}_{V}} \int_{\mathbf{B}_{V}}\left(1+\left\|D \overline{\mathbf{y}}_{x+\rho|x| z}\right\|_{\mathrm{HS}}^{2}\right)^{\frac{1}{2}}-1 d x .
\end{aligned}
$$

As in (3.8), for fixed $z \in \mathbf{B}_{V}(0,1)$ the map $\psi: x \mapsto x+\rho|x| z$ is a bijection of $\mathbf{B}_{V}(0,1)$ and a subset of $\mathbf{B}_{V}(0,1+\rho)$ with $D \psi_{x}(v)=v+\frac{\rho}{|x|}\langle x, v\rangle z$ and $\operatorname{det} D \psi_{x}=1+\frac{\rho}{|x|}\langle x, z\rangle$ for $x \neq 0$. It is $\operatorname{det} D \psi_{x} \geq 1-\rho$ and since we assume that $\rho \leq \frac{1}{2}$ we obtain

$$
\operatorname{det} D\left(\psi^{-1}\right)_{\xi} \leq \frac{1}{1-\rho} \leq 1+2 \rho
$$

for almost all $\xi \in \psi\left(\mathbf{B}_{V}(0,1)\right)$. By the homogeneity of $\|D \overline{\mathbf{y}}\|_{\mathrm{HS}}$,

$$
\begin{aligned}
\int_{\mathbf{B}_{V}(0,1)}(1 & \left.+\left\|D \overline{\mathbf{y}}_{x+\rho|x| z}\right\|_{\mathrm{HS}}^{2}\right)^{\frac{1}{2}}-1 d x \\
& =\int_{\psi\left(\mathbf{B}_{V}(0,1)\right)}\left(\left(1+\left\|D \overline{\mathbf{y}}_{\xi}\right\|_{\mathrm{HS}}^{2}\right)^{\frac{1}{2}}-1\right) \operatorname{det} D\left(\psi^{-1}\right)_{\xi} d \xi \\
& \leq(1+2 \rho) \int_{\mathbf{B}_{V}(0,1+\rho)}\left(1+\left\|D \overline{\mathbf{y}}_{\xi}\right\|_{\mathrm{HS}}^{2}\right)^{\frac{1}{2}}-1 d \xi \\
& =(1+2 \rho)(1+\rho)^{m} \int_{\mathbf{B}_{V}(0,1)}\left(1+\left\|D \overline{\mathbf{y}}_{\xi}\right\|_{\mathrm{HS}}^{2}\right)^{\frac{1}{2}}-1 d \xi \\
& \leq\left(1+\mathbf{c}^{\prime \prime} \rho\right) \int_{\mathbf{B}_{V}(0,1)}\left(1+\left\|D \overline{\mathbf{y}}_{\xi}\right\|_{\mathrm{HS}}^{2}\right)^{\frac{1}{2}}-1 d \xi
\end{aligned}
$$


for some constant $\mathbf{c}^{\prime \prime}(m)>0$. By the excess bound in term of $\epsilon$, we obtain as in $(3.17)$,

$$
\begin{aligned}
\int_{\mathbf{B}_{V}}\left(1+\|D \overline{\mathbf{y}}\|_{\mathrm{HS}}^{2}\right)^{\frac{1}{2}}-1 & \leq \int_{\mathbf{B}_{V}}\left(1+\left(\sum_{i \in I_{\xi}} \delta_{i}(\xi)\left\|D \mathbf{y}_{\xi}^{i}\right\|_{\mathrm{HS}}\right)^{2}\right)^{\frac{1}{2}}-1 d \xi \\
& \leq \int_{\mathbf{B}_{V}} \sum_{i \in I_{\xi}} \delta_{i}(\xi)\left(1+\left\|D \mathbf{y}_{\xi}^{i}\right\|_{\mathrm{HS}}^{2}\right)^{\frac{1}{2}}-1 d \xi \\
& \leq\left\|g_{0}\right\|^{-1} \int_{\mathbf{B}_{V}} \sum_{i \in I_{\xi}}\left\|g_{i}\right\|\left(1+\left(\mathcal{J} \mathbf{y}_{\xi}^{i}\right)^{2}\right)^{\frac{1}{2}}-\left\|g_{0}\right\| d \xi \\
& =\left\|g_{0}\right\|^{-1} \operatorname{Exc}_{1}(P, V) \leq \epsilon .
\end{aligned}
$$

Combining (3.19),(3.20) and (3.21) we see that for $\mathbf{c}^{\prime \prime \prime}=\max \left\{\mathbf{c}^{\prime \prime}, \mathbf{c}^{\prime}\right\}$,

$$
\begin{aligned}
\int_{\mathbf{B}_{V}}\left(1+\|D \mathbf{v}\|_{\mathrm{HS}}^{2}\right)^{\frac{1}{2}}-1 & \leq\left(1+\mathbf{c}^{\prime \prime \prime} \rho\right)^{4} \int_{\mathbf{B}_{V}}\left(1+\left\|D \overline{\mathbf{y}}_{\xi}\right\|_{\mathrm{HS}}^{2}\right)^{\frac{1}{2}}-1 d \xi \\
& \leq \mathbf{c}^{\prime \prime \prime \prime \prime} \rho \epsilon+\int_{\mathbf{B}_{V}}\left(1+\left\|D \overline{\mathbf{y}}_{\xi}\right\|_{\mathrm{HS}}^{2}\right)^{\frac{1}{2}}-1 d \xi
\end{aligned}
$$

for some constant $\mathbf{c}^{\prime \prime \prime \prime}(m)>0$. With (3.5) of Lemma 3.1 we obtain,

$$
\int_{\mathbf{B}_{V}}\left(1+\|D \mathbf{v}\|_{\mathrm{HS}}^{2}\right)^{\frac{1}{2}} \leq \mathbf{c}^{\prime \prime \prime \prime} \rho \epsilon+\int_{\mathbf{B}_{V}}\left(\sum_{j \in I_{\xi}}\left\|g_{j}\right\|\right)^{-1} \sum_{i \in I_{\xi}}\left\|g_{i}\right\|\left(1+\left\|D \mathbf{y}_{\xi}^{i}\right\|_{\mathrm{HS}}^{2}\right)^{\frac{1}{2}} d \xi .
$$

Applying (3.3), Lemma 3.7 and $\epsilon^{\frac{2}{3}} \leq \rho$ to the estimate above leads to,

$$
\begin{aligned}
\int_{\mathbf{B}_{V}} & \sum_{i \in I_{x}}\left\|g_{i}\right\|\left(\left(1+\left\|D \mathbf{v}_{x}\right\|^{2}\right)^{\frac{1}{2}}-1\right) d x \\
\leq & \left\|g_{0}\right\| \int_{E_{1}}\left(1+\left\|D \mathbf{v}_{x}\right\|_{\mathrm{HS}}^{2}\right)^{\frac{1}{2}}-1 d x+\int_{E_{2}} \sum_{i \in I_{x}}\left\|g_{i}\right\|\left\|D \mathbf{v}_{x}\right\|_{\mathrm{HS}}^{2} d x \\
\leq & \left\|g_{0}\right\| \int_{\mathbf{B}_{V}}\left(1+\left\|D \mathbf{v}_{x}\right\|_{\mathrm{HS}}^{2}\right)^{\frac{1}{2}}-1 d x+15\left\|g_{0}\right\| \epsilon \mathbf{c}_{3.7} \epsilon^{\frac{2}{3}} \\
\leq & 15 \mathbf{c}_{3.7}\left\|g_{0}\right\| \epsilon^{\frac{5}{3}}+\mathbf{c}^{\prime \prime \prime \prime}\left\|g_{0}\right\| \rho \epsilon \\
& +\left\|g_{0}\right\| \int_{\mathbf{B}_{V}}\left(\sum_{j \in I_{x}}\left\|g_{j}\right\|\right)^{-1} \sum_{i \in I_{x}}\left\|g_{i}\right\|\left(\left(1+\left\|D \mathbf{y}_{x}^{i}\right\|_{\mathrm{HS}}^{2}\right)^{\frac{1}{2}}-1\right) d x \\
\leq & \left(15 \mathbf{c}_{3.7}+\mathbf{c}^{\prime \prime \prime \prime \prime}\right)\left\|g_{0}\right\| \rho \epsilon+\int_{\mathbf{B}_{V}} \sum_{i \in I_{x}}\left\|g_{i}\right\|\left(\left(1+\left\|D \mathbf{y}_{x}^{i}\right\|_{\mathrm{HS}}^{2}\right)^{\frac{1}{2}}-1\right) d x .
\end{aligned}
$$

Adding $\int_{\mathbf{B}_{V}} \sum_{i \in I_{x}}\left\|g_{i}\right\|$ to both sides, the lemma follows.

By assumption (4) we have $\pi_{V \#}\left(P\left\llcorner Z_{V}\right)=g_{0} \llbracket \mathbf{B}_{V}(0,1) \rrbracket\right.$. Define the rectifiable $G$-chain $T^{\prime}:=\left(\operatorname{id}_{V}+\mathbf{v}\right)_{\#}\left(g_{0} \llbracket \mathbf{B}_{V}(0,1) \rrbracket\right) \in \mathscr{R}_{m}(X ; G)$. Lemma 3.5 implies that

$$
d_{\mathrm{H}}\left(\left(\operatorname{spt}(P) \cup \operatorname{spt}\left(T^{\prime}\right)\right) \cap Z_{V}, \mathbf{B}_{V}(0,1)\right) \leq 2 \rho<1 .
$$

Let $\varphi: X \rightarrow X$ be some Lipschitz map with the following properties: for $x \in V$ and $y \in V^{\perp}$,

(1) $\varphi(x+y)=x+y$, if $|x| \geq \frac{3}{4}$ or $|y| \geq 2$,

(2) $\varphi(x+y)=x+\mathbf{v}(x)$, if $|x| \leq \frac{1}{2}$ and $|y| \leq 1$, 
(3) $\varphi(x+y)=x+(4|x|-2) y+(3-4|x|) \mathbf{v}(x))$, if $\frac{1}{2} \leq|x| \leq \frac{3}{4}$ and $|y| \leq 1$,

Define $T:=\varphi_{\#} P \in \mathscr{R}_{m}(X ; G)$. It is easy to check that

(1) $\partial\left(T\left\llcorner Z_{V}\left(\frac{3}{4}\right)\right)=\partial\left(P\left\llcorner Z_{V}\left(\frac{3}{4}\right)\right)\right.\right.$,

(2) $\pi_{V \#}\left(T\left\llcorner Z_{V}\right)=g_{0} \llbracket \mathbf{B}_{V}(0,1) \rrbracket\right.$,

(3) $T\left\llcorner Z_{V}\left(\frac{1}{2}\right)=T^{\prime}\left\llcorner Z_{V}\left(\frac{1}{2}\right)\right.\right.$,

(4) for $\frac{1}{2} \leq|x| \leq \frac{3}{4}$,

$$
\operatorname{spt}(T) \cap \pi_{V}^{-1}(x)=\bigcup_{i \in I_{x}}\left\{x+(3-4|x|) \mathbf{v}(x)+(4|x|-2) \mathbf{y}^{i}(x)\right\} .
$$

3.9. Lemma ([20, Lemma 11]). There are constants $\mathbf{c}_{3.9}(m), \rho_{3.9}(m), \epsilon_{3.9}(m)>0$ such that if $0<\rho \leq \rho_{3.9}(\mathrm{~m})$ and $0<\epsilon \leq \epsilon_{3.9}(\mathrm{~m})$ (recall we also always assume $\left.\epsilon \leq \rho^{6 m}\right)$, then

$$
\int_{A} \sum_{i \in I_{x}}\left\|g_{i}\right\|\left(1+\left(\mathcal{J} \mathbf{z}_{x}^{i}\right)^{2}\right)^{\frac{1}{2}} d x \leq \mathbf{c}_{3.9}\left\|g_{0}\right\| \rho^{\frac{1}{2}} \epsilon+\int_{A} \sum_{i \in I_{x}}\left\|g_{i}\right\|\left(1+\left(\mathcal{J} \mathbf{y}_{x}^{i}\right)^{2}\right)^{\frac{1}{2}} d x
$$

where $\mathbf{z}^{i}:=(4 r-2) \mathbf{y}^{i}+(3-4 r) \mathbf{v}, r(x):=|x|$ and $A:=\left\{x \in V: \frac{1}{2} \leq|x| \leq \frac{3}{4}\right\}$.

Proof. We start by noticing that $0 \leq 4|x|-2 \leq 1$ as well as $0 \leq 3-4|x| \leq 1$, two facts we will freely use without further reference in the course of this proof. For almost all $x \in A$ it is

$$
D \mathbf{z}_{x}^{i}(v)=(4|x|-2) D \mathbf{y}_{x}^{i}(v)+(3-4|x|) D \mathbf{v}_{x}(v)+\frac{4\langle x, v\rangle}{|x|}\left(\mathbf{y}^{i}(x)-\mathbf{v}(x)\right) .
$$

Fix a point $x$ for which all the differentials in the formula above exist and consider the matrix $M_{k l}^{i}:=\left\langle D \mathbf{z}_{x}^{i}\left(e_{k}\right), D \mathbf{z}_{x}^{i}\left(e_{l}\right)\right\rangle$ for some orthonormal basis $\left(e_{1}, \ldots, e_{m}\right)$ of $V$. Applying (2.5),

$$
\left(\mathcal{J} \mathbf{z}_{x}^{i}\right)^{2}=\sum_{K \neq \emptyset} \operatorname{det}\left(M_{K}^{i}\right)
$$

For simplicity we assume that the $e_{k}=e_{k}^{i}$ 's are eigenvectors of $D \mathbf{y}_{x}^{i *} D \mathbf{y}_{x}^{i}$ with eigenvalues $\left(\lambda_{k}^{i}\right)^{2}$, for $\lambda_{k}^{i} \geq 0$, and $\Lambda^{i}$ is the corresponding diagonal matrix (i.e. $\left.\Lambda_{k l}^{i}=\left\langle D \mathbf{y}_{x}^{i}\left(e_{k}^{i}\right), D \mathbf{y}_{x}^{i}\left(e_{l}^{i}\right)\right\rangle=\left(\lambda_{k}^{i}\right)^{2} \delta_{k l}\right)$. We can write

$$
M_{k l}^{i}=(4|x|-2)^{2} \Lambda_{k l}^{i}+(3-4|x|)^{2}\left\langle D \mathbf{v}_{x}\left(e_{k}^{i}\right), D \mathbf{v}_{x}\left(e_{l}^{i}\right)\right\rangle+R_{k l}^{i},
$$

where

$$
\begin{aligned}
\left|R_{k l}^{i}\right| \leq & 16\left|\mathbf{y}^{i}(x)-\mathbf{v}(x)\right|^{2}+8\left\|D \mathbf{v}_{x}\right\|\left|\mathbf{y}^{i}(x)-\mathbf{v}(x)\right| \\
& +\left(\left\|D \mathbf{v}_{x}\right\|+4\left|\mathbf{y}^{i}(x)-\mathbf{v}(x)\right|\right)\left(\lambda_{k}^{i}+\lambda_{l}^{i}\right) .
\end{aligned}
$$

Next we estimate the minors of $M^{i}$ corresponding to some nonempty subset $K \subset$ $\{1, \ldots, m\}$. Using (3.22) to expand each $M_{k k}^{i}=\operatorname{det}\left(M_{\{k\}}^{i}\right)$ we find that for subsets 
with one element $K=\{k\}$,

$$
\begin{aligned}
\left\|D \mathbf{z}_{x}^{i}\right\|_{\mathrm{HS}}^{2}= & \sum_{k=1}^{m} \operatorname{det}\left(M_{\{k\}}^{i}\right) \\
\leq & (4|x|-2)^{2} \sum_{k=1}^{m} \Lambda_{k k}^{i}+(3-4|x|)^{2} \sum_{k=1}^{m}\left|D \mathbf{v}_{x}\left(e_{k}\right)\right|^{2} \\
& \quad+2(4|x|-2)(3-4|x|) \sum_{k=1}^{m}\left\langle D \mathbf{y}_{x}^{i}\left(e_{k}\right), D \mathbf{v}_{x}\left(e_{k}\right)\right\rangle \\
& +16\left|\mathbf{y}^{i}(x)-\mathbf{v}(x)\right|^{2}+8\left|\mathbf{y}^{i}(x)-\mathbf{v}(x)\right|\left(\left\|D \mathbf{v}_{x}\right\|+\left\|D \mathbf{y}_{x}^{i}\right\|\right) \\
= & \left((4|x|-2)\left\|D \mathbf{y}_{x}^{i}\right\|_{\mathrm{HS}}+(3-4|x|)\left\|D \mathbf{v}_{x}\right\|_{\mathrm{HS}}\right)^{2} \\
& +16\left|\mathbf{y}^{i}(x)-\mathbf{v}(x)\right|^{2}+8\left|\mathbf{y}^{i}(x)-\mathbf{v}(x)\right|\left(\left\|D \mathbf{v}_{x}\right\|+\left\|D \mathbf{y}_{x}^{i}\right\|\right) .
\end{aligned}
$$

Furthermore the exists a constant $\mathbf{c}=\mathbf{c}(m)>0$ such that if $\# K \geq 2$ then,

$$
\begin{aligned}
\operatorname{det}\left(M_{K}^{i}\right) \leq & (4|x|-2)^{2} \operatorname{det}\left(\Lambda_{K}^{i}\right)+\mathbf{c}\left\|D \mathbf{v}_{x}\right\|^{4} \\
& +\mathbf{c}\left(\left|\mathbf{y}^{i}(x)-\mathbf{v}(x)\right|^{2}+\left\|D \mathbf{v}_{x}\right\|\left|\mathbf{y}^{i}(x)-\mathbf{v}(x)\right|\right) \\
& +\mathbf{c}\left(\left|\mathbf{y}^{i}(x)-\mathbf{v}(x)\right|+\left\|D \mathbf{v}_{x}\right\|\right)\left(\mathcal{J} \mathbf{y}_{x}^{i}\right)^{2}
\end{aligned}
$$

We now indicate how to obtain the above inequality:

$$
\begin{aligned}
& \operatorname{det}\left(M_{K}^{i}\right)=\sum_{\sigma \in S_{q}}(-1)^{|\sigma|} \prod_{k \in K} M_{k \sigma(k)}^{i} \\
& =\sum_{\sigma \in S_{q}}(-1)^{|\sigma|} \prod_{k \in K}\left((4|x|-2)^{2} \Lambda_{k \sigma(k)}^{i}+(3-4|x|)^{2}\left\langle D \mathbf{v}_{x}\left(e_{k}^{i}\right), D \mathbf{v}_{x}\left(e_{\sigma(k)}^{i}\right)\right\rangle+R_{k \sigma(k)}^{i}\right) \\
& =\sum_{\sigma \in S_{q}}(-1)^{|\sigma|} \sum_{K_{1}, K_{2}, K_{3}}\left(\prod_{k \in K_{1}}(4|x|-2)^{2} \Lambda_{k \sigma(k)}^{i}\right) \\
& \left(\prod_{k \in K_{2}}(3-4|x|)^{2}\left\langle D \mathbf{v}_{x}\left(e_{k}^{i}\right), D \mathbf{v}_{x}\left(e_{\sigma(k)}^{i}\right)\right\rangle\right)\left(\prod_{k \in K_{3}} R_{k \sigma(k)}^{i}\right),
\end{aligned}
$$

where the last sum extends over all partitions $\left\{K_{1}, K_{2}, K_{3}\right\}$ of $K$. Letting $q:=\# K$, the term corresponding to $K_{2}=K_{3}=\emptyset$ equals $(4|x|-2)^{2 q} \operatorname{det}\left(\Lambda_{K}^{i}\right)$ when $\sigma=\operatorname{id}_{K}$ and zero otherwise, whereas the term corresponding to $K_{1}=K_{3}=\emptyset$ is bounded above by $(3-4|x|)^{2 q}\left\|D \mathbf{v}_{x}\right\|^{2 q}$ regardless of $\sigma$. These are respectively bounded above by $(4|x|-2)^{2} \operatorname{det}\left(\Lambda_{K}^{i}\right)$ and by $\left\|D \mathbf{v}_{x}\right\|^{4}$ since $0 \leq \operatorname{det}\left(\Lambda_{K}^{i}\right)$, since $q \geq 2$, and since we may assume $\left\|D \mathbf{v}_{x}\right\| \leq \mathbf{c}_{3.7}(m) \epsilon^{\frac{1}{3}} \leq 1$ provided $\epsilon$ is small enough depending upon $\epsilon$. Thus these two terms, corresponding to all $\sigma \in S_{q}$, are accounted for in the first line of (3.25). In order to bound from above the other terms, we start by observing that if $\emptyset \neq \hat{K} \subset\{1, \ldots, m\}$ then

$$
\prod_{k \in \hat{K}}\left(\lambda_{k}^{i}\right)^{2} \leq \operatorname{det}\left(I+\Lambda^{i}\right)-1=\left(\mathcal{J} \mathbf{y}_{x}^{i}\right)^{2},
$$

Furthermore a product $\prod_{k \in \hat{K}} \Lambda_{k \sigma(k)}^{i}$ is either zero (when $\sigma \neq \mathrm{id}_{K}$ ) or equal to $\prod_{k \in \hat{K}}\left(\lambda_{k}^{i}\right)^{2}$, thus in both cases it is less than or equal to $\left(\mathcal{J} \mathbf{y}_{x}^{i}\right)^{2}$. Now the remaining 
terms corresponding to $K_{3}=\emptyset$ have necessarily $K_{1} \neq \emptyset \neq K_{2}$ and are therefore bounded above by

$$
\left(\prod_{k \in K_{1}} \Lambda_{k \sigma(k)}^{i}\right)\left(\prod_{k \in K_{2}}\left\|D \mathbf{v}_{x}\right\|^{2}\right) \leq\left\|D \mathbf{v}_{x}\right\|\left(\mathcal{J} \mathbf{y}_{x}^{i}\right)^{2}
$$

and are accounted for in the last line of (3.25). Next we consider the terms corresponding to $K_{3} \neq \emptyset$ and we define $a_{x}^{i}$ and $b_{x}^{i}$ in the obvious way, according to (3.23), so that $\left|R_{k l}^{i}\right| \leq a_{x}^{i}+b_{x}^{i}\left(\lambda_{k}^{i}+\lambda_{l}^{i}\right)$ and we notice that we may assume $0 \leq a_{x}^{i} \leq 1$ and $0 \leq b_{x}^{i} \leq 1$ provided $\epsilon$ is small enough depending on $m$. Now

$$
\begin{aligned}
\left|\prod_{k \in K_{3}} R_{k \sigma(k)}^{i}\right| \leq & \prod_{k \in K_{3}}\left(a_{b}^{i}+b_{x}^{i}\left(\lambda_{k}^{i}+\lambda_{\sigma(k)}^{i}\right)\right) \\
= & \sum_{K_{3,1}, K_{3,2}}\left(a_{x}^{i}\right)^{\# K_{3,1}}\left(b_{x}^{i}\right)^{\# K_{3,2}} \prod_{k \in K_{3,2}}\left(\lambda_{k}^{i}+\lambda_{\sigma(k)}^{i}\right) \\
& \leq\left\{\begin{array}{ll}
a_{x}^{i} & \text { if } K_{3,2}=\emptyset \\
b_{x}^{i} \prod_{k \in K_{3,2}}\left(\lambda_{k}^{i}+\lambda_{\sigma(k)}^{i}\right) & \text { if } K_{3,2} \neq \emptyset
\end{array},\right.
\end{aligned}
$$

where the sum extends over partitions $\left\{K_{3,1}, K_{3,2}\right\}$ of $K_{3}$. Thus the terms corresponding to $K_{3} \neq \emptyset, K_{3,2}=\emptyset$ and $K_{1}=\emptyset$ are accounted for in the second line of (3.25), whereas the terms where $K_{3} \neq \emptyset, K_{3,2}=\emptyset$ and $K_{1} \neq \emptyset$ are accounted for in the third line of (3.25) because $\left|\mathbf{y}^{i}(x)-\mathbf{v}(x)\right| \leq 1$. It remains to consider the terms for which $K_{3,2} \neq \emptyset$. The following sum extends over partitions $\left\{L_{1}, L_{2}\right\}$ of $K_{3,2}$ :

$$
\begin{aligned}
\prod_{k \in K_{3,2}}\left(\lambda_{k}^{i}+\lambda_{\sigma(k)}^{i}\right) & =\sum_{L_{1}, L_{2}}\left(\prod_{k \in L_{1}} \lambda_{k}^{i}\right)\left(\prod_{k \in \sigma\left(L_{2}\right)} \lambda_{k}^{i}\right) \\
& =\sum_{L_{1}, L_{2}}\left(\prod_{k \in L_{1} \cap \sigma\left(L_{2}\right)}\left(\lambda_{k}^{i}\right)^{2}\right)\left(\prod_{k \in L_{1} \ominus \sigma\left(L_{2}\right)} \lambda_{k}^{i}\right) \\
& \leq \sum_{L_{1}, L_{2}}\left(\prod_{k \in L_{1} \cap \sigma\left(L_{2}\right)}\left(\lambda_{k}^{i}\right)^{2}\right)\left(\prod_{\substack{k \in L_{1} \ominus \sigma\left(L_{2}\right) \\
\lambda_{k}^{i} \geq 1}}\left(\lambda_{k}^{i}\right)^{2}\right)
\end{aligned}
$$

We first analyze the various subcases occurring as $K_{1}=\emptyset$. If $L_{1} \cap \sigma\left(L_{2}\right) \neq \emptyset$, the corresponding term is bounded above by $b_{x}^{i}\left(\mathcal{J} \mathbf{y}_{x}^{i}\right)^{2}$ and thus is accounted for in the third line of (3.25). The same occurs if $L_{1} \cap \sigma\left(L_{2}\right)=\emptyset$ but there exists $k \in L_{1} \ominus \sigma\left(L_{2}\right)$ such that $\lambda_{k}^{i} \geq 1$. Now if $\lambda_{k}^{i}<1$ for all $k \in L_{1} \ominus \sigma\left(L_{2}\right)$, and $L_{1} \cap \sigma\left(L_{2}\right)=\emptyset$, we pick two $\lambda_{k_{1}}^{i}, \lambda_{k_{2}}^{i} \in L_{1} \ominus \sigma\left(L_{2}\right)$ with $k_{1} \neq k_{2}$ (this is possible since \# $\left(L_{1} \ominus \sigma\left(L_{2}\right)\right) \geq 2$ because $\left.L_{1} \cap \sigma\left(L_{2}\right)=\emptyset\right)$ and we observe that we can bound a factor $\lambda_{k_{1}}^{i} \lambda_{k_{2}}^{i} \leq \frac{1}{2}\left(\left(\lambda_{k_{1}}^{i}\right)^{2}+\left(\lambda_{k_{2}}^{i}\right)^{2}\right) \leq\left(\mathcal{J} \mathbf{y}_{x}^{i}\right)^{2}$ and, again, the corresponding term is bounded above by $b_{x}^{i}\left(\mathcal{J} \mathbf{y}_{x}^{i}\right)^{2}$ and thus is accounted for in the third line of (3.25). 
Finally it remains only to consider the case when $K_{3,2} \neq \emptyset$ and $K_{1} \neq \emptyset$. We observe these terms are bounded above by

$$
b_{x}^{i}\left(\prod_{k \in K_{1}} \Lambda_{k \sigma(k)}^{i}\right)\left(\prod_{k \in L_{1} \cap \sigma\left(L_{2}\right)}\left(\lambda_{k}^{i}\right)^{2}\right)\left(\prod_{\substack{k \in L_{1} \ominus \sigma\left(L_{2}\right) \\ \lambda_{k}^{i} \geq 1}}\left(\lambda_{k}^{i}\right)^{2}\right)
$$

Clearly $K_{1} \cap L_{1} \subset K_{1} \cap K_{3}=\emptyset$. If $\sigma_{K_{1}} \neq \mathrm{id}_{K_{1}}$ then the second factor above (corresponding to $k \in K_{1}$ ) vanishes, thus we may assume $K_{1} \cap \sigma\left(L_{2}\right)=\emptyset$ but then the product is of the type $b_{x}^{i} \prod_{k \in \hat{K}}\left(\lambda_{k}^{i}\right)^{2}$ with $\hat{K} \neq \emptyset$ and (3.25) is established.

Summing up (3.25) over all $K$ with $\# K \geq 2$ we find that

$$
\begin{aligned}
\left(\mathcal{J} \mathbf{z}_{x}^{i}\right)^{2}-\left\|D \mathbf{z}_{x}^{i}\right\|_{\mathrm{HS}}^{2}= & \sum_{\substack{K \subset\{1, \ldots, m\} \\
\# K \geq 2}} \operatorname{det}\left(M_{K}^{i}\right) \\
\leq & (4|x|-2)^{2}\left(\left(\mathcal{J} \mathbf{y}_{x}^{i}\right)^{2}-\left\|D \mathbf{y}_{x}^{i}\right\|_{\mathrm{HS}}^{2}\right)+\mathbf{c}^{\prime}\left\|D \mathbf{v}_{x}\right\|^{4} \\
& +\mathbf{c}^{\prime}\left(\left|\mathbf{y}^{i}(x)-\mathbf{v}(x)\right|^{2}+\left\|D \mathbf{v}_{x}\right\|\left|\mathbf{y}^{i}(x)-\mathbf{v}(x)\right|\right) \\
& +\mathbf{c}^{\prime}\left(\left|\mathbf{y}^{i}(x)-\mathbf{v}(x)\right|+\left\|D \mathbf{v}_{x}\right\|\right)\left(\mathcal{J} \mathbf{y}_{x}^{i}\right)^{2},
\end{aligned}
$$

and with the help of (3.24), recalling that $\left\|D \mathbf{y}_{x}^{i}\right\|_{\mathrm{HS}} \leq \mathcal{J} \mathbf{y}_{x}^{i}$,

$$
\begin{aligned}
\left(\mathcal{J} \mathbf{z}_{x}^{i}\right)^{2} \leq(4|x| & -2)^{2}\left(\left(\mathcal{J} \mathbf{y}_{x}^{i}\right)^{2}-\left\|D \mathbf{y}_{x}^{i}\right\|_{\mathrm{HS}}^{2}\right) \\
& +\left((4|x|-2)\left\|D \mathbf{y}_{x}^{i}\right\|_{\mathrm{HS}}+(3-4|x|)\left\|D \mathbf{v}_{x}\right\|_{\mathrm{HS}}\right)^{2}+\mathbf{c}^{\prime}\left\|D \mathbf{v}_{x}\right\|^{4} \\
& +\mathbf{c}^{\prime}\left(\left|\mathbf{y}^{i}(x)-\mathbf{v}(x)\right|^{2}+\left\|D \mathbf{v}_{x}\right\|\left|\mathbf{y}^{i}(x)-\mathbf{v}(x)\right|\right) \\
& +\mathbf{c}^{\prime}\left(\left|\mathbf{y}^{i}(x)-\mathbf{v}(x)\right|+\left\|D \mathbf{v}_{x}\right\|\right)\left(\mathcal{J} \mathbf{y}_{x}^{i}\right)^{2} \\
& +\mathbf{c}^{\prime}\left|\mathbf{y}^{i}(x)-\mathbf{v}(x)\right|\left\|D \mathbf{y}_{x}^{i}\right\| \\
\leq((4|x| & \left.-2) \mathcal{J} \mathbf{y}_{x}^{i}+(3-4|x|)\left\|D \mathbf{v}_{x}\right\|_{\mathrm{HS}}\right)^{2}+\mathbf{c}^{\prime}\left\|D \mathbf{v}_{x}\right\|^{4} \\
& +\mathbf{c}^{\prime}\left(\left|\mathbf{y}^{i}(x)-\mathbf{v}(x)\right|^{2}+\left\|D \mathbf{v}_{x}\right\|\left|\mathbf{y}^{i}(x)-\mathbf{v}(x)\right|\right) \\
& +\mathbf{c}^{\prime}\left(\left|\mathbf{y}^{i}(x)-\mathbf{v}(x)\right|+\left\|D \mathbf{v}_{x}\right\|\right)\left(\mathcal{J} \mathbf{y}_{x}^{i}\right)^{2} \\
& +\mathbf{c}^{\prime}\left|\mathbf{y}^{i}(x)-\mathbf{v}(x)\right|\left\|D \mathbf{y}_{x}^{i}\right\| \\
=((4|x| & \left.-2) \mathcal{J} \mathbf{y}_{x}^{i}+(3-4|x|)\left\|D \mathbf{v}_{x}\right\|_{\mathrm{HS}}\right)^{2}+\sum_{j=1}^{6} R^{i j}(x)
\end{aligned}
$$

for some $\mathbf{c}^{\prime}(m)>0$, where the $R^{i j}(x), j=1, \ldots, 6$, are readily defined by the last equality.

Now from the above inequality, applying seven times (3.4) of Lemma 3.1, and one time (3.5), we see that

$$
\begin{aligned}
\left(1+\left(\mathcal{J} \mathbf{z}_{x}^{i}\right)^{2}\right)^{\frac{1}{2}} \leq(4|x|-2)\left(1+\left(\mathcal{J} \mathbf{y}_{x}^{i}\right)^{2}\right)^{\frac{1}{2}} & +(3-4|x|)\left(1+\left\|D \mathbf{v}_{x}\right\|_{\mathrm{HS}}^{2}\right)^{\frac{1}{2}} \\
& +\sum_{j=1}^{6}\left(\left(1+R^{i j}(x)\right)^{\frac{1}{2}}-1\right) .
\end{aligned}
$$


Since the maps $x \mapsto\left\|g_{i}(x)\right\|, x \mapsto\left\|D \mathbf{v}_{x}\right\|_{\text {HS }}$ and $x \mapsto \mathcal{J} \mathbf{y}_{x}^{i}$ are 0-homogeneous for all $i$ we obtain, thanks to Lemma 3.8,

$$
\begin{aligned}
\int_{|x|=r} \sum_{i \in I_{x}}\left\|g_{i}\right\| & \left(1+\left\|D \mathbf{v}_{x}\right\|_{\mathrm{HS}}^{2}\right)^{\frac{1}{2}} d \mathscr{H}^{m-1}(x) \\
= & \frac{d}{d r} \int_{\mathbf{B}_{V}(0, r)} \sum_{i \in I_{x}}\left\|g_{i}\right\|\left(1+\left\|D \mathbf{v}_{x}\right\|_{\mathrm{HS}}^{2}\right)^{\frac{1}{2}} d x \\
& =m r^{m-1} \int_{\mathbf{B}_{V}(0,1)} \sum_{i \in I_{x}}\left\|g_{i}\right\|\left(1+\left\|D \mathbf{v}_{x}\right\|_{\mathrm{HS}}^{2}\right)^{\frac{1}{2}} d x \\
& \leq m r^{m-1} \int_{\mathbf{B}_{V}(0,1)} \sum_{i \in I_{x}}\left\|g_{i}\right\|\left(1+\left(\mathcal{J} \mathbf{y}_{x}^{i}\right)^{2}\right)^{\frac{1}{2}} d x+m r^{m-1} \mathbf{c}_{3.8}\left\|g_{0}\right\| \rho \epsilon \\
& =\int_{|x|=r} \sum_{i \in I_{x}}\left\|g_{i}\right\|\left(1+\left(\mathcal{J} \mathbf{y}_{x}^{i}\right)^{2}\right)^{\frac{1}{2}} d \mathscr{H}^{m-1}(x)+m r^{m-1} \mathbf{c}_{3.8}\left\|g_{0}\right\| \rho \epsilon .
\end{aligned}
$$

Therefore,

$$
\begin{aligned}
\int_{A} \sum_{i \in I_{x}}\left\|g_{i}\right\|\left((4|x|-2)\left(1+\left(\mathcal{J} \mathbf{y}_{x}^{i}\right)^{2}\right)^{\frac{1}{2}}+(3-4|x|)\left(1+\left\|D \mathbf{v}_{x}\right\|_{\mathrm{HS}}^{2}\right)^{\frac{1}{2}}\right) d x \\
=\int_{\frac{1}{2}}^{\frac{3}{4}}\left((4 r-2) \int_{|x|=r} \sum_{i \in I_{x}}\left\|g_{i}\right\|\left(1+\left(\mathcal{J} \mathbf{y}_{x}^{i}\right)^{2}\right)^{\frac{1}{2}} d \mathscr{H}^{m-1}(x)\right. \\
\left.\quad+(3-4 r) \int_{|x|=r} \sum_{i \in I_{x}}\left\|g_{i}\right\|\left(1+\left\|D \mathbf{v}_{x}\right\|_{\mathrm{HS}}^{2}\right)^{\frac{1}{2}} d \mathscr{H}^{m-1}(x)\right) d r \\
\leq \int_{\frac{1}{2}}^{\frac{3}{4}}\left((4 r-2) \int_{|x|=r} \sum_{i \in I_{x}}\left\|g_{i}\right\|\left(1+\left(\mathcal{J} \mathbf{y}_{x}^{i}\right)^{2}\right)^{\frac{1}{2}} d \mathscr{H}^{m-1}(x)\right. \\
\quad+(3-4 r) \int_{|x|=r} \sum_{i \in I_{x}}\left\|g_{i}\right\|\left(1+\left(\mathcal{J} \mathbf{y}_{x}^{i}\right)^{2}\right)^{\frac{1}{2}} d \mathscr{H}^{m-1}(x) \\
\left.\quad+(3-4 r) m r^{m-1} \mathbf{c}_{3.8}\left\|g_{0}\right\| \rho \epsilon\right) d r \\
\leq m \mathbf{c}_{3.8}\left\|g_{0}\right\| \rho \epsilon+\int_{A} \sum_{i \in I_{x}}\left\|g_{i}\right\|\left(1+\left(\mathcal{J} \mathbf{y}_{x}^{i}\right)^{2}\right)^{\frac{1}{2}} d x
\end{aligned}
$$

Thus it follows from (3.26) that

$$
\begin{array}{r}
\int_{A} \sum_{i \in I_{x}}\left\|g_{i}\right\|\left(1+\left(\mathcal{J} \mathbf{z}_{x}^{i}\right)^{2}\right)^{\frac{1}{2}} d x \leq m \mathbf{c}_{3.8}\left\|g_{0}\right\| \rho \epsilon+\int_{A} \sum_{i \in I_{x}}\left\|g_{i}\right\|\left(1+\left(\mathcal{J} \mathbf{y}_{x}^{i}\right)^{2}\right)^{\frac{1}{2}} d x \\
+\sum_{j=1}^{6} \int_{A} \sum_{i \in I_{x}}\left\|g_{i}\right\|\left(\left(1+R^{i j}(x)\right)^{\frac{1}{2}}-1\right) d x .
\end{array}
$$

In order to complete the proof of the Lemma it is therefore sufficient to establish that

$$
\int_{A} \sum_{i \in I_{x}}\left\|g_{i}\right\|\left(\left(1+R^{i j}(x)\right)^{\frac{1}{2}}-1\right) d x \leq \mathbf{c}^{\prime \prime \prime}(m)\left\|g_{0}\right\| \rho^{\frac{1}{2}} \epsilon
$$

for some $\mathbf{c}^{\prime \prime \prime}(m)>0$ and all $j=1, \ldots, 6$. 
Clearly,

$$
\begin{aligned}
& \left(1+R^{i 1}(x)\right)^{\frac{1}{2}}-1 \leq R^{i 1}(x) \leq \mathbf{c}^{\prime}\left\|D \mathbf{v}_{x}\right\|^{4} \leq \mathbf{c}^{\prime} \mathbf{c}_{3.7^{4}}^{\epsilon^{\frac{4}{3}}} \\
& \left(1+R^{i 2}(x)\right)^{\frac{1}{2}}-1 \leq R^{i 2}(x) \leq \mathbf{c}^{\prime}\left|\mathbf{y}^{i}(x)-\mathbf{v}(x)\right|^{2}
\end{aligned}
$$

Setting $\delta:=\rho^{\frac{1}{2}}<1$ we obtain with (3.7) of Lemma 3.1,

$$
\begin{aligned}
\left(1+R^{i 3}(x)\right)^{\frac{1}{2}}-1 & =\left(1+\mathbf{c}^{\prime}\left\|D \mathbf{v}_{x}\right\|\left|\mathbf{y}^{i}(x)-\mathbf{v}(x)\right|\right)^{\frac{1}{2}}-1 \\
& \leq \frac{1}{2} \delta^{-2} \mathbf{c}^{\prime 2}\left|\mathbf{y}^{i}(x)-\mathbf{v}(x)\right|^{2}+\delta\left(\left(1+\left\|D \mathbf{v}_{x}\right\|^{2}\right)^{\frac{1}{2}}-1\right) \\
& \leq \frac{1}{2} \mathbf{c}^{\prime 2} \rho^{-1}\left|\mathbf{y}^{i}(x)-\mathbf{v}(x)\right|^{2}+\rho^{\frac{1}{2}}\left(\left(1+\left\|D \mathbf{v}_{x}\right\|^{2}\right)^{\frac{1}{2}}-1\right)
\end{aligned}
$$

Setting $\delta:=\mathbf{c}^{\prime}\left|\mathbf{y}^{i}(x)-\mathbf{v}(x)\right| \leq \mathbf{c}^{\prime} 3 \rho \leq 1$ provided $\rho$ is small enough depending on $m$, we infer from (3.6) of Lemma 3.1 that

$$
\begin{aligned}
\left(1+R^{i 4}(x)\right)^{\frac{1}{2}}-1 & =\left(1+\mathbf{c}^{\prime}\left|\mathbf{y}^{i}(x)-\mathbf{v}(x)\right|\left(\mathcal{J} \mathbf{y}_{x}^{i}\right)^{2}\right)^{\frac{1}{2}}-1 \\
& \leq\left(3 \mathbf{c}^{\prime} \rho\right)^{\frac{1}{2}}\left(\left(1+\left(\mathcal{J} \mathbf{y}_{x}^{i}\right)^{2}\right)^{\frac{1}{2}}-1\right)
\end{aligned}
$$

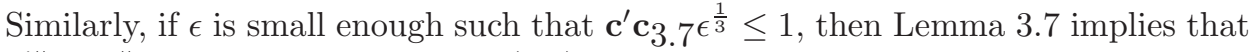
$\mathbf{c}^{\prime}\left\|D \mathbf{v}_{x}\right\| \leq 1$ and it follows from (3.6) of Lemma 3.1 that

$$
\begin{aligned}
\left(1+R^{i 5}(x)\right)^{\frac{1}{2}}-1 & =\left(1+\mathbf{c}^{\prime}\left\|D \mathbf{v}_{x}\right\|\left(\mathcal{J} \mathbf{y}_{x}^{i}\right)^{2}\right)^{\frac{1}{2}}-1 \\
& \leq\left(\mathbf{c}^{\prime} \mathbf{c}_{3.7}\right)^{\frac{1}{2}} \epsilon^{\frac{1}{6}}\left(\left(1+\left(\mathcal{J} \mathbf{y}_{x}^{i}\right)^{2}\right)^{\frac{1}{2}}-1\right)
\end{aligned}
$$

Finally, applying again (3.7) of Lemma 3.1 with $\delta:=\rho^{\frac{1}{2}}$,

$$
\begin{aligned}
\left(1+R^{i 6}(x)\right)^{\frac{1}{2}}-1 & =\left(1+\mathbf{c}^{\prime}\left|\mathbf{y}^{i}(x)-\mathbf{v}(x)\right|\left\|D \mathbf{y}_{x}^{i}\right\|\right)^{\frac{1}{2}}-1, \\
& \leq \frac{1}{2} \delta^{-2} \mathbf{c}^{\prime 2}\left|\mathbf{y}^{i}(x)-\mathbf{v}(x)\right|^{2}+\delta\left(\left(1+\left\|D \mathbf{y}_{x}^{i}\right\|^{2}\right)^{\frac{1}{2}}-1\right), \\
& \leq \frac{1}{2} \mathbf{c}^{\prime 2} \rho^{-1}\left|\mathbf{y}^{i}(x)-\mathbf{v}(x)\right|^{2}+\rho^{\frac{1}{2}}\left(\left(1+\left(\mathcal{J} \mathbf{y}_{x}^{i}\right)^{2}\right)^{\frac{1}{2}}-1\right) .
\end{aligned}
$$

We next integrate these over $x \in A$. By (3.3),

$$
\begin{aligned}
\int_{A} \sum_{i \in I_{x}}\left\|g_{i}\right\|\left[\left(1+R^{i 1}(x)\right)^{\frac{1}{2}}-1\right] d x & \leq \mathbf{c}^{\prime} \epsilon^{\frac{4}{3}} \int_{A} \sum_{i \in I_{x}}\left\|g_{i}\right\| d x \\
& =\mathbf{c}^{\prime} \epsilon^{\frac{4}{3}} \int_{E_{1}}\left\|g_{0}\right\| d x+\mathbf{c}^{\prime} \epsilon^{\frac{4}{3}} 15\left\|g_{0}\right\| \epsilon \\
& \leq(\boldsymbol{\alpha}(m)+15) \mathbf{c}^{\prime}\left\|g_{0}\right\| \epsilon^{\frac{4}{3}} .
\end{aligned}
$$

For the remaining terms note that by (3.3) and Lemma 3.5,

$$
\begin{aligned}
\int_{A} \sum_{i \in I_{x}}\left\|g_{i}\right\|\left|\mathbf{v}(x)-\mathbf{y}^{i}(x)\right|^{2} d x & \leq \int_{E_{1}}\left\|g_{0}\right\||\mathbf{y}(x)-\mathbf{v}(x)|^{2} d x+9 \rho^{2} \int_{E_{2}} \sum_{i \in I_{x}}\left\|g_{i}\right\| d x \\
& \leq\left(\mathbf{c}_{3.5}+135\right)\left\|g_{0}\right\| \rho^{2} \epsilon .
\end{aligned}
$$


Further by Lemma 3.8,

$$
\begin{aligned}
\int_{A} \sum_{i \in I_{x}}\left\|g_{i}\right\| & \left(\left(1+\left\|D \mathbf{v}_{x}\right\|^{2}\right)^{\frac{1}{2}}-1\right) d x \\
& \leq \mathbf{c}_{3.8}\left\|g_{0}\right\| \rho \epsilon+\int_{\mathbf{B}_{V}} \sum_{i \in I_{x}}\left\|g_{i}\right\|\left(\left(1+\left\|D \mathbf{y}_{x}^{i}\right\|^{2}\right)^{\frac{1}{2}}-1\right) d x \\
& \leq \mathbf{c}_{3.8}\left\|g_{0}\right\| \rho \epsilon+\int_{\mathbf{B}_{V}} \sum_{i \in I_{x}}\left\|g_{i}\right\|\left(1+\left\|\mathcal{J} \mathbf{y}_{x}^{i}\right\|^{2}\right)^{\frac{1}{2}}-\left\|g_{0}\right\| d x \\
& \leq\left(\mathbf{c}_{3.8} \rho+1\right)\left\|g_{0}\right\| \epsilon
\end{aligned}
$$

and obviously,

$$
\begin{aligned}
\int_{A} \sum_{i \in I_{x}}\left\|g_{i}\right\|\left(\left(1+\left(\mathcal{J} \mathbf{y}_{x}^{i}\right)^{2}\right)^{\frac{1}{2}}-1\right) d x & \leq \int_{\mathbf{B}_{V}} \sum_{i \in I_{x}}\left\|g_{i}\right\|\left(1+\left(\mathcal{J} \mathbf{y}_{x}^{i}\right)^{2}\right)^{\frac{1}{2}}-\left\|g_{0}\right\| d x \\
& \leq\left\|g_{0}\right\| \epsilon
\end{aligned}
$$

Combining these estimates we obtain

$$
\begin{aligned}
& \int_{A} \sum_{i \in I_{x}}\left\|g_{i}\right\|\left[\left(1+R^{i 1}(x)\right)^{\frac{1}{2}}-1\right] d x \leq \mathbf{c}^{\prime \prime}(m)\left\|g_{0}\right\| \epsilon^{\frac{4}{3}}, \\
& \int_{A} \sum_{i \in I_{x}}\left\|g_{i}\right\|\left[\left(1+R^{i 2}(x)\right)^{\frac{1}{2}}-1\right] d x \leq \mathbf{c}^{\prime \prime}(m)\left\|g_{0}\right\| \rho^{2} \epsilon, \\
& \int_{A} \sum_{i \in I_{x}}\left\|g_{i}\right\|\left[\left(1+R^{i 3}(x)\right)^{\frac{1}{2}}-1\right] d x \leq \mathbf{c}^{\prime \prime}(m)\left\|g_{0}\right\| \rho^{\frac{1}{2}} \epsilon, \\
& \int_{A} \sum_{i \in I_{x}}\left\|g_{i}\right\|\left[\left(1+R^{i 4}(x)\right)^{\frac{1}{2}}-1\right] d x \leq \mathbf{c}^{\prime \prime}(m)\left\|g_{0}\right\| \rho^{\frac{1}{2}} \epsilon, \\
& \int_{A} \sum_{i \in I_{x}}\left\|g_{i}\right\|\left[\left(1+R^{i 5}(x)\right)^{\frac{1}{2}}-1\right] d x \leq \mathbf{c}^{\prime \prime}(m)\left\|g_{0}\right\| \epsilon^{\frac{7}{6}}, \\
& \int_{A} \sum_{i \in I_{x}}\left\|g_{i}\right\|\left[\left(1+R^{i 6}(x)\right)^{\frac{1}{2}}-1\right] d x \leq \mathbf{c}^{\prime \prime}(m)\left\|g_{0}\right\| \rho^{\frac{1}{2}} \epsilon,
\end{aligned}
$$

for some constant $\mathbf{c}^{\prime \prime}(m)>0$. Since we assume that $\epsilon \leq \rho^{6 m} \leq \rho^{6} \leq 1$, the sum of all the integrals involving the $R$-terms is bounded by $\mathbf{c}^{\prime \prime \prime}(m)\left\|g_{0}\right\| \rho^{\frac{1}{2}} \epsilon$ for some constant $\mathbf{c}^{\prime \prime \prime}(m)>0$.

3.10. Lemma ([20, Lemma 12]). There is a constant $\mathbf{c}_{3.10}(m)>0$ such that

$$
\int_{B_{V}(0,1)}\left(1+\left(\mathcal{J} \mathbf{v}_{x}\right)^{2}\right)^{\frac{1}{2}} d x \leq \int_{B_{V}(0,1)}\left(1+\left\|D \mathbf{v}_{x}\right\|_{\mathrm{HS}}^{2}\right)^{\frac{1}{2}} d x+\mathbf{c}_{3.10} \epsilon^{\frac{4}{3}} .
$$

Proof. By Lemma 3.7 we know that $\left\|D \mathbf{v}_{x}\right\| \leq \mathbf{c}_{3.7} \epsilon^{\frac{1}{3}}$. Hence letting $M$ denote the matrix of $D \mathbf{v}_{x}^{*} D \mathbf{v}_{x}$ with respect to some orthonormal basis of $V$,

$$
\left(\mathcal{J} \mathbf{v}_{x}\right)^{2}=\left\|D \mathbf{v}_{x}\right\|_{\mathrm{HS}}^{2}+\sum_{\# K \geq 2} \operatorname{det}\left(M_{K}\right) \leq\left\|D \mathbf{v}_{x}\right\|_{\mathrm{HS}}^{2}+\mathbf{c} \epsilon^{\frac{4}{3}}
$$

for some constant $\mathbf{c}(m)>0$, and the statement follows by Lemma 3.1, i.e. by integrating the estimate

$$
\left(1+\left(\mathcal{J} \mathbf{v}_{x}\right)^{2}\right)^{\frac{1}{2}} \leq\left(1+\left\|D \mathbf{v}_{x}\right\|_{\mathrm{HS}}^{2}+\mathbf{c} \epsilon^{\frac{4}{3}}\right)^{\frac{1}{2}} \leq\left(1+\left\|D \mathbf{v}_{x}\right\|_{\mathrm{HS}}^{2}\right)^{\frac{1}{2}}+\mathbf{c} \epsilon^{\frac{4}{3}} .
$$


Combining the results of this subsection we get:

3.11. Proposition. There is a constant $\mathbf{c}_{3.11}(m)>0$ with the following property. If $\rho \leq \rho_{3.9}$ and $\epsilon \leq \epsilon_{3.9}$, then

$$
\begin{aligned}
\mathbf{M}\left(T\left\llcorner Z_{V}\left(2^{-1}\right)\right)\right. & \leq \mathbf{c}_{3.11}\left\|g_{0}\right\| \rho \epsilon+\mathbf{M}\left(P\left\llcorner Z_{V}\left(2^{-1}\right)\right),\right. \\
\mathbf{M}(T) & \leq \mathbf{c}_{3.11}\left\|g_{0}\right\| \rho^{\frac{1}{2}} \epsilon+\mathbf{M}(P) .
\end{aligned}
$$

Proof. By the construction of $T$, it is $T\left\llcorner Z_{V}\left(\frac{3}{4}\right)^{c}=P\left\llcorner Z_{V}\left(\frac{3}{4}\right)^{c}\right.\right.$. By Lemma 3.9

$$
\mathbf{M}\left(T\left\llcorner\left\{\frac{1}{2} \leq\left|\pi_{V}(x)\right| \leq \frac{3}{4}\right\}\right) \leq \mathbf{M}\left(P\left\llcorner\left\{\frac{1}{2} \leq\left|\pi_{V}(x)\right| \leq \frac{3}{4}\right\}\right)+\mathbf{c}_{3.9}\left\|g_{0}\right\| \rho^{\frac{1}{2}} \epsilon .\right.\right.
$$

Further, by the definition of $T$, Lemma 3.10 and Lemma 3.8 (note that $\epsilon^{\frac{1}{3}} \leq \rho$ ) applied to the integration domain $\mathbf{B}_{V}(0,1)$,

$$
\begin{aligned}
\mathbf{M}\left(T\left\llcorner Z_{V}\left(2^{-1}\right)\right)\right. & =\mathbf{M}\left(\left(\operatorname{id}_{V}+\mathbf{v}\right)_{\#}\left(g_{0} \llbracket \mathbf{B}_{V}\left(0,2^{-1}\right) \rrbracket\right)\right. \\
& =\left\|g_{0}\right\| \int_{\mathbf{B}_{V}\left(0,2^{-1}\right)}\left(1+\left(\mathcal{J} \mathbf{v}_{x}\right)^{2}\right)^{\frac{1}{2}} d x \\
& \leq\left\|g_{0}\right\| \int_{\mathbf{B}_{V}\left(0,2^{-1}\right)}\left(1+\left\|D \mathbf{v}_{x}\right\|_{\mathrm{HS}}^{2}\right)^{\frac{1}{2}} d x+\mathbf{c}_{3.10}\left\|g_{0}\right\| \epsilon^{\frac{4}{3}} \\
& \leq \int_{\mathbf{B}_{V}\left(0,2^{-1}\right)} \sum_{i \in I_{x}}\left\|g_{i}\right\|\left(1+\left\|D \mathbf{y}_{x}^{i}\right\|_{\mathrm{HS}}^{2}\right)^{\frac{1}{2}} d x+\left(\mathbf{c}_{3.10}+\mathbf{c}_{3.8}\right) \rho\left\|g_{0}\right\| \epsilon \\
& \leq \int_{\mathbf{B}_{V}\left(0,2^{-1}\right)} \sum_{i \in I_{x}}\left\|g_{i}\right\|\left(1+\left(\mathcal{J} \mathbf{y}_{x}^{i}\right)^{2}\right)^{\frac{1}{2}} d x+\left(\mathbf{c}_{3.10}+\mathbf{c}_{3.8}\right) \rho\left\|g_{0}\right\| \epsilon \\
& =\mathbf{M}\left(P\left\llcorner Z_{V}\left(2^{-1}\right)\right)+\left(\mathbf{c}_{3.10}+\mathbf{c}_{3.8}\right) \rho\left\|g_{0}\right\| \epsilon .\right.
\end{aligned}
$$

Adding up the masses of these two regions gives the result.

3.3. Plane selection via a quadratic form. Additionally to the assumptions on $P$ in the beginning of Subsection 3.1 we further assume that $\rho \leq \rho_{3.9}$ (without mentioning it repeatedly) and that $\epsilon \leq \epsilon_{3.9}$. With $T_{\mathbf{v}}:=\left(\operatorname{id}_{V}+\mathbf{v}\right)_{\#}\left(g_{0} \llbracket \mathbf{B}_{V}(0,2) \rrbracket\right)$ we denote the cone generated by the graph of $\mathbf{v}$ over $V$ and weight $g_{0}$. Let $Q=Q\left(T_{\mathbf{v}}, 0,1\right)$ be the quadratic form associated to the chain $T_{\mathbf{v}}$ as defined in Subsection 2.5, i.e.

$$
Q(y):=\frac{m+2}{\boldsymbol{\alpha}(m)}\left\|g_{0}\right\| \int_{\mathbf{B}(0,1) \cap \operatorname{spt}\left(T_{\mathbf{v}}\right)}\langle y, x\rangle^{2} d \mathscr{H}^{m}(x) .
$$

As noted before Lemma 2.12, the quadratic form $Q$ is compact and hence $X$ has an orthonormal basis $\left(e_{k}\right)_{k}$ of eigenvectors of $Q$. Let $W \in \mathbf{G}(X, m)$ be the $m$-plane spanned by $e_{1}, \ldots, e_{m}$ corresponding to the $m$ largest eigenvalues of $Q$.

3.12. Lemma. There are constants $\mathbf{c}_{3.12}(m)>0$ and $0<\epsilon_{3.12} \leq \epsilon_{3.9}$ with $\mathbf{c}_{3.12}\left(\epsilon_{3.12}\right)^{\frac{1}{3}}<\frac{1}{20}$ and the following property. If $0<\epsilon<\epsilon_{3.12}$, then:

(1) $\left\|\pi_{W}-\pi_{V}\right\| \leq \mathbf{c}_{3.12} \epsilon^{\frac{1}{3}}$.

(2) $\pi_{W}: \operatorname{spt}\left(T_{\mathbf{v}}\right) \rightarrow W$ is injective and the map $\mathbf{w}: \boldsymbol{B}_{W}(0,1) \rightarrow W^{\perp}$ with $\operatorname{graph}(\mathbf{w})=\operatorname{spt}\left(T_{\mathbf{v}}\left\llcorner Z_{W}\right)\right.$ is 1-homogeneous, continuously differentiable in $\boldsymbol{B}_{W}(0,1) \backslash\{0\}$ with $\left\|D \mathbf{w}_{x}\right\| \leq \mathbf{c}_{3.12} \epsilon^{\frac{1}{3}}$. 
(3) For $x \in \boldsymbol{B}_{W}(0,1), y \in W^{\perp}$ with $|x|,|y| \leq 1$,

$$
\left|\int_{S_{W}}\langle y, \mathbf{w}(z)\rangle\langle x, z\rangle d \mathscr{H}^{m-1}(z)\right| \leq \mathbf{c}_{3.12} \epsilon
$$

Proof. By Lemma 3.7 we have a height bound for $T_{\mathbf{v}}$ over $\mathbf{B}_{V}(0,1)$ of $\mathbf{c}_{3.7^{\epsilon^{\frac{1}{3}}} \text { and by }}$ Proposition 3.11 the excess is bounded by $\operatorname{Exc}_{1}\left(T_{\mathbf{v}}, V\right) \leq 2^{m} \mathbf{c}_{3.11}\left\|g_{0}\right\| \rho \epsilon+\left\|g_{0}\right\| \epsilon$. The first statement is now a direct consequence of Lemma 2.12 and there is some $\mathbf{c}(m)>0$ such that $\left\|\pi_{W}-\pi_{V}\right\| \leq \mathbf{c} \epsilon^{\frac{1}{3}}$. We may assume that $\epsilon$ is small enough such that $\max \left\{\mathbf{c}, \mathbf{c}_{3.7}\right\} \epsilon^{\frac{1}{3}} \leq \frac{1}{4}$. For $v \in V$ we have $\left|v-\pi_{W}(v)\right| \leq \mathbf{c} \epsilon^{\frac{1}{3}}|v| \leq \frac{1}{2}|v|$, respectively, $\frac{1}{2}|v| \leq\left|\pi_{W}(v)\right|$. Now, consider two points $p, p^{\prime} \in \operatorname{spt}\left(T_{\mathbf{v}}\right)$, i.e. $p=$ $v+\mathbf{v}(v)$ and $p^{\prime}=v^{\prime}+\mathbf{v}\left(v^{\prime}\right)$ for $v, v^{\prime} \in V$. By Lemma 3.7, $\mathbf{v}$ is Lipschitz with constant $\mathbf{c}_{3.7^{\frac{1}{3}}}$ and hence

$$
\begin{aligned}
\left|\pi_{W^{\perp}}\left(p-p^{\prime}\right)\right| & =\left|p-p^{\prime}-\pi_{W}\left(p-p^{\prime}\right)\right| \\
& \leq\left|v-v^{\prime}-\pi_{W}\left(v-v^{\prime}\right)\right|+\left|\mathbf{v}(v)-\mathbf{v}\left(v^{\prime}\right)-\pi_{W}\left(\mathbf{v}(v)-\mathbf{v}\left(v^{\prime}\right)\right)\right| \\
& \leq \mathbf{c} \epsilon^{\frac{1}{3}}\left|v-v^{\prime}\right|+2 \mathbf{c}_{3.7^{\frac{1}{3}}}\left|v-v^{\prime}\right| \\
& \leq 2\left(\mathbf{c}+2 \mathbf{c}_{3.7}\right) \epsilon^{\frac{1}{3}}\left|\pi_{W}\left(v-v^{\prime}\right)\right| .
\end{aligned}
$$

Also

$$
\begin{aligned}
& \left|\pi_{W}\left(p-p^{\prime}\right)\right| \geq\left|\pi_{W}\left(v-v^{\prime}\right)\right|-\left|\pi_{W}\left(\mathbf{v}(v)-\mathbf{v}\left(v^{\prime}\right)\right)\right| \\
& \geq\left|\pi_{W}\left(v-v^{\prime}\right)\right|-\mathbf{c}_{3.7^{\epsilon^{\frac{1}{3}}}}\left|v-v^{\prime}\right|
\end{aligned}
$$

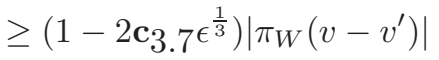

$$
\begin{aligned}
& \geq \frac{1}{2}\left|\pi_{W}\left(v-v^{\prime}\right)\right| \text {. }
\end{aligned}
$$

Combined we get

$$
\left|\pi_{W^{\perp}}\left(p-p^{\prime}\right)\right| \leq \mathbf{c}^{\prime} \epsilon^{\frac{1}{3}}\left|\pi_{W}\left(p-p^{\prime}\right)\right|,
$$

for some constant $\mathbf{c}^{\prime}(m)>0$. This in turn implies that $\mathbf{w}$ is well defined and Lipschitz with the constant as stated in (2). Since $\pi_{W} \circ\left(\mathrm{id}_{V}+\mathbf{v}\right)$ is of class $C^{1}$ outside the origin, it follows from the inverse function theorem that the same holds for $\mathbf{w}$ and $\left\|D \mathbf{w}_{x}\right\| \leq \operatorname{Lip}(\mathbf{w}) \leq \mathbf{c}^{\prime} \epsilon^{\frac{1}{3}}$. This shows (2).

Let $D:=\mathbf{B}(0,1) \cap \operatorname{spt}\left(T_{\mathbf{v}}\right)$. For $x \in W$ and $y \in W^{\perp}$ with $|x|,|y| \leq 1$,

$$
\begin{aligned}
0 & =\frac{\boldsymbol{\alpha}(m)}{\left\|g_{0}\right\|(m+2)} Q(x, y)=\int_{D}\langle x, z\rangle\langle y, z\rangle d \mathscr{H}^{m}(z) \\
& =\int_{\pi_{W}(D)}\langle x, w+\mathbf{w}(w)\rangle\langle y, w+\mathbf{w}(w)\rangle\left(1+\left(\mathcal{J} \mathbf{w}_{w}\right)^{2}\right)^{\frac{1}{2}} d w \\
& =\int_{\pi_{W}(D)}\langle x, w\rangle\langle y, \mathbf{w}(w)\rangle\left(1+\left(\mathcal{J} \mathbf{w}_{w}\right)^{2}\right)^{\frac{1}{2}} d w .
\end{aligned}
$$

Because of $\left\|D \mathbf{w}_{x}\right\| \leq \mathbf{c}^{\prime} \epsilon^{\frac{1}{3}}$, we note as in the proof of Lemma 3.10 that

$$
1 \leq\left(1+\left(\mathcal{J} \mathbf{w}_{z}\right)^{2}\right)^{\frac{1}{2}} \leq 1+\mathbf{c}^{\prime \prime} \epsilon^{\frac{2}{3}},
$$

for some constant $\mathbf{c}^{\prime \prime}(m)>0$. Hence $|s(w)| \leq \mathbf{c}^{\prime \prime} \epsilon^{\frac{2}{3}}$ for $s(w):=\left(1+\left(\mathcal{J} \mathbf{w}_{w}\right)^{2}\right)^{\frac{1}{2}}-1$ and $w \in \mathbf{B}_{W}(0,1)$. Further, $\mathscr{H}^{m}\left(\mathbf{B}_{W}(0,1) \backslash \pi_{W}(D)\right) \leq \mathbf{c}^{\prime} \epsilon^{\frac{2}{3}}$ since the height bound 
obtained above implies $\mathbf{B}_{W}\left(0, \sqrt{1-\mathbf{c}^{\prime 2} \epsilon^{\frac{2}{3}}}\right) \subset \pi_{W}(D)$ and $|\mathbf{w}(w)| \leq \mathbf{c}^{\prime} \epsilon^{\frac{1}{3}} \leq 1$ if $\epsilon$ is small enough. Hence,

$$
\begin{aligned}
\left|\int_{\mathbf{B}_{W}(0,1)}\langle y, \mathbf{w}(w)\rangle\langle x, w\rangle d w\right| \\
\leq\left|\int_{\mathbf{B}_{W}(0,1)}\langle y, \mathbf{w}(w)\rangle\langle x, w\rangle(1+s(w)) d w\right| \\
\quad+\left|\int_{\mathbf{B}_{W}(0,1)}\langle y, \mathbf{w}(w)\rangle\langle x, w\rangle s(w) d w\right| \\
\leq\left|\int_{\mathbf{B}_{W}(0,1) \backslash \pi_{W}(D)}\langle y, \mathbf{w}(w)\rangle\langle x, w\rangle(1+s(w)) d w\right|+\boldsymbol{\alpha}(m)\|\mathbf{w}\|_{\infty}\left\|_{s}\right\|_{\infty} \\
\leq \mathscr{H}^{m}\left(\mathbf{B}_{W}(0,1) \backslash \pi_{W}(D)\right)\|\mathbf{w}\|_{\infty} 2+\boldsymbol{\alpha}(m)\|\mathbf{w}\|_{\infty}\|s\|_{\infty} \\
\leq \mathbf{c}^{\prime \prime \prime} \epsilon
\end{aligned}
$$

for some constant $\mathbf{c}^{\prime \prime \prime}(m)>0$. By the homogeneity of $\mathbf{w}$ the last statement of the lemma follows.

Let

$$
\mathbf{w}_{0}:=\frac{1}{\boldsymbol{\alpha}(m) m} \int_{\mathbf{S}_{W}} \mathbf{w}(x) d \mathscr{H}^{m-1}(x) \in W^{\perp},
$$

and note that $\boldsymbol{\alpha}(m) m=\mathscr{H}^{m-1}\left(\mathbf{S}_{W}\right)$. Instead of taking the harmonic extension of w over $\mathbf{S}_{W}$ as in [20], we follow [17] and define $\mathbf{h}: \mathbf{B}_{W}(0,1) \rightarrow W^{\perp}$ by

$$
\mathbf{h}(t x):=\mathbf{w}_{0}+t^{2}\left(\mathbf{w}(x)-\mathbf{w}_{0}\right),
$$

for $|x|=1$ and $t \in[0,1]$. For a point $x \in \mathbf{S}_{W}$ we denote with $D_{S} \mathbf{w}_{x}$ the derivative of the restriction of $\mathbf{w}$ to $\mathbf{S}_{W}$ at the point $x$. The following calculations are contained in [17].

3.13. Lemma. There is a constant $\mathbf{c}_{3.13}(m)>0$ such that if $0<\epsilon<\epsilon_{3.12}$, then

$$
\int_{B_{W}(0,1)}\left(1+(\mathcal{J} \mathbf{h})^{2}\right)^{\frac{1}{2}} \leq \mathbf{c}_{3.13} \epsilon^{\frac{4}{3}}+\int_{B_{W}(0,1)}\left(1+\|D \mathbf{h}\|_{\mathrm{HS}}^{2}\right)^{\frac{1}{2}}
$$

and further

$$
\begin{aligned}
& \int_{B_{W}(0,1)}\|D \mathbf{h}\|_{\mathrm{HS}}^{2}=\frac{1}{m+2} \int_{S_{W}} 4\left|\mathbf{w}(x)-\mathbf{w}_{0}\right|^{2}+\left\|D_{S} \mathbf{w}_{x}\right\|_{\mathrm{HS}}^{2} d \mathscr{H}^{m-1}(x), \\
& \int_{B_{W}(0,1)}\|D \mathbf{w}\|_{\mathrm{HS}}^{2}=\frac{1}{m} \int_{S_{W}}|\mathbf{w}(x)|^{2}+\left\|D_{S} \mathbf{w}_{x}\right\|_{\mathrm{HS}}^{2} d \mathscr{H}^{m-1}(x) .
\end{aligned}
$$

Proof. By definition and the 1-homogeneity of $\mathbf{w}$ it is

$$
\mathbf{h}(x)=\mathbf{w}_{0}+|x|^{2}\left(\mathbf{w}(x /|x|)-\mathbf{w}_{0}\right)=\mathbf{w}_{0}\left(1-|x|^{2}\right)+|x| \mathbf{w}(x) .
$$

Hence,

$$
D \mathbf{h}_{x}(w)=-2\langle x, w\rangle \mathbf{w}_{0}+\langle x /|x|, w\rangle \mathbf{w}(x)+|x| D \mathbf{w}_{x}(w) .
$$

If $x \neq 0$ and $e_{1}, \ldots, e_{m}$ is an orthonormal basis of $W$ with $e_{1}=|x|^{-1} x$, then $|x| D \mathbf{w}_{x}\left(e_{1}\right)=\mathbf{w}(x)$ and further

$$
\begin{aligned}
D \mathbf{h}_{x}\left(e_{1}\right) & =-2|x| \mathbf{w}_{0}+\mathbf{w}(x)+|x| D \mathbf{w}_{x}\left(e_{1}\right)=2\left(\mathbf{w}(x)-|x| \mathbf{w}_{0}\right) \\
& =2|x|\left(\mathbf{w}(x /|x|)-\mathbf{w}_{0}\right) .
\end{aligned}
$$


For $k \geq 2$ by the 1-homogeneity of $\mathbf{w}$,

$$
D \mathbf{h}_{x}\left(e_{k}\right)=|x| D \mathbf{w}_{x}\left(e_{k}\right)=|x| D_{S} \mathbf{w}_{|x|^{-1} x}\left(e_{k}\right) .
$$

Hence,

$$
\begin{aligned}
\left\|D \mathbf{h}_{x}\right\|_{\mathrm{HS}}^{2} & =\sum_{k}\left|D \mathbf{h}_{x}\left(e_{k}\right)\right|^{2} \\
& =\left|D \mathbf{h}_{x}\left(e_{1}\right)\right|^{2}+|x|^{2} \sum_{k \geq 2}\left|D_{S} \mathbf{w}_{x /|x|}\left(e_{k}\right)\right|^{2} \\
& =4|x|^{2}\left|\mathbf{w}(x /|x|)-\mathbf{w}_{0}\right|^{2}+|x|^{2}\left\|D_{S} \mathbf{w}_{x /|x|}\right\|_{\mathrm{HS}}^{2},
\end{aligned}
$$

and in particular with the definition of $\mathbf{w}_{0}$ and Lemma 3.12,

$$
\left\|D \mathbf{h}_{x}\right\|_{\mathrm{HS}}^{2} \leq 16 \operatorname{Lip}(\mathbf{w})^{2}+\left\|D \mathbf{w}_{x}\right\|_{\mathrm{HS}}^{2} \leq 17 \mathbf{c}_{3.12^{\epsilon^{\frac{2}{3}}}} .
$$

The first statement follows now exactly as in Lemma 3.10. Similarly,

$$
\left\|D \mathbf{w}_{x}\right\|_{\mathrm{HS}}^{2}=|\mathbf{w}(x /|x|)|^{2}+\left\|D_{S} \mathbf{w}_{x /|x|}\right\|_{\mathrm{HS}}^{2} .
$$

Integrating gives

$$
\begin{aligned}
\int_{\mathbf{B}_{W}(0,1)}\|D \mathbf{h}\|_{\mathrm{HS}}^{2} & =\int_{0}^{1} d r \int_{\mathbf{S}_{W}(r)}\left\|D \mathbf{h}_{x}\right\|_{\mathrm{HS}}^{2} d \mathscr{H}^{m-1}(x) \\
& =\int_{0}^{1} r^{2} d r \int_{\mathbf{S}_{W}(r)} 4\left|\mathbf{w}(x / r)-\mathbf{w}_{0}\right|^{2}+\left\|D_{S} \mathbf{w}_{x / r}\right\|_{\mathrm{HS}}^{2} d \mathscr{H}^{m-1}(x) \\
& =\int_{0}^{1} r^{m+1} d r \int_{\mathbf{S}_{W}} 4\left|\mathbf{w}(x)-\mathbf{w}_{0}\right|^{2}+\left\|D_{S} \mathbf{w}_{x}\right\|_{\mathrm{HS}}^{2} d \mathscr{H}^{m-1}(x) \\
& =\frac{1}{m+2} \int_{\mathbf{S}_{W}} 4\left|\mathbf{w}(x)-\mathbf{w}_{0}\right|^{2}+\left\|D_{S} \mathbf{w}_{x}\right\|_{\mathrm{HS}}^{2} d \mathscr{H}^{m-1}(x)
\end{aligned}
$$

And similarly,

$$
\begin{aligned}
\int_{\mathbf{B}_{V}(0,1)}\|D \mathbf{w}\|_{\mathrm{HS}}^{2} & =\int_{0}^{1} r^{m-1} d r \int_{\mathbf{S}_{W}}|\mathbf{w}(x)|^{2}+\left\|D_{S} \mathbf{w}_{x}\right\|_{\mathrm{HS}}^{2} d \mathscr{H}^{m-1}(x) \\
& =\frac{1}{m} \int_{\mathbf{S}_{W}}|\mathbf{w}(x)|^{2}+\left\|D_{S} \mathbf{w}_{x}\right\|_{\mathrm{HS}}^{2} d \mathscr{H}^{m-1}(x) .
\end{aligned}
$$

It is $L^{2}\left(\mathbf{S}_{W}\right)=\oplus_{i=0}^{\infty} \mathcal{H}_{i}\left(\mathbf{S}_{W}\right)$ where $\mathcal{H}_{i}\left(\mathbf{S}_{W}\right)$ denotes the Hilbert space of harmonic polynomials of degree $i$ on $\mathbf{S}_{W}=\mathbf{S}^{m-1}$. Hence for a fixed orthonormal basis $\left(e_{l}\right)_{l>m}$ of $W^{\perp}$ we can write the restriction $w^{l}=\left\langle\mathbf{w}, e_{l}\right\rangle$ as a sum $\sum_{i \geq 0} w_{i}^{l} p_{i}^{l}$ with $w_{i}^{l} \in \mathbb{R}$ and $p_{i}^{l} \in \mathcal{H}_{i}\left(\mathbf{S}_{W}\right)$ of unit $L^{2}$-norm. It is understood that the partial sums converge in $L^{2}$ to $w^{l}: \mathbf{B}_{W}(0,1) \rightarrow \mathbb{R}$. Every $p_{i}^{l}$ is the restriction of a harmonic homogeneous polynomial of degree $i$ (the extension is just given by $p_{i}^{l}(r x)=r^{i} p_{i}^{l}(x)$ for $r \geq 0$ ), which is for simplicity also denoted by $p_{i}^{l}$. Thus

$$
\begin{aligned}
\int_{\mathbf{S}_{W}}|\mathbf{w}(x)|^{2} & =\int_{\mathbf{S}_{W}} \sum_{m<l}\left|w^{l}(x)\right|^{2}=\sum_{m<l} \int_{\mathbf{S}_{W}}\left|w^{l}(x)\right|^{2} \\
& =\sum_{m<l} \sum_{i=0}^{\infty} \int_{\mathbf{S}_{W}}\left(w_{i}^{l}\right)^{2}\left|p_{i}^{l}(x)\right|^{2}=\sum_{m<l} \sum_{i=0}^{\infty}\left(w_{i}^{l}\right)^{2} \\
& \leq \boldsymbol{\alpha}(m) m \mathbf{c}_{3.12^{2}}^{\epsilon^{\frac{2}{3}}},
\end{aligned}
$$


if we assume that $\epsilon$ is small enough. In particular, for each fixed $i, \sum_{m<l}\left(w_{i}^{l}\right)^{2}<$ $\infty$. Furthermore, since $\mathcal{H}_{i}\left(\mathbf{S}_{W}\right)$ is finite dimensional, there is a constant $C(i, m)$ depending only on $i$ and $m$ such that $\left|p_{i}^{l}(x)\right| \leq C(i, m)$ for all $|x| \leq 1$. Hence,

$$
\sup _{|x| \leq 1}\left|\sum_{m<N<l \leq N^{\prime}} w_{i}^{l} p_{i}^{l}(x) e_{l}\right|^{2} \leq C(i, m)^{2} \sum_{m<N<l \leq N^{\prime}}\left(w_{i}^{l}\right)^{2} \rightarrow 0,
$$

as $N, N^{\prime} \rightarrow \infty$, and the partial sums

$$
\left(x \mapsto \sum_{m<l \leq N} w_{i}^{l} p_{i}^{l}(x) e_{l}\right)_{N>m}
$$

converge uniformly with respect to the norm in $X$ for $N \rightarrow \infty$ to some $\mathbf{w}_{i}$ : $\mathbf{B}_{W}(0,1) \rightarrow W^{\perp}$. Similarly, the norms of the derivatives up to some fixed order of these partial sums converge uniformly as well, hence $\mathbf{w}_{i}$ is smooth because the partial sums are. Note that $\mathbf{w}_{0}$ is the constant map with value as defined in (3.28). Finally, it is not hard to see that the partial sums $\sum_{i} \mathbf{w}_{i}$ converge to $\mathbf{w}$ in $L^{2}\left(\mathbf{S}_{W}, W^{\perp}\right)$.

3.14. Lemma. There is a constant $\mathbf{c}_{3.14}(m)>0$ with the following property. If $0<\epsilon<\epsilon 3.12$, then $\left\|\mathbf{w}_{1}\right\|_{\infty}:=\sup _{|x| \leq 1}\left|\mathbf{w}_{1}(x)\right| \leq \mathbf{c}_{3.14}(m) \epsilon$.

Proof. For $i \neq 1, x \in W$ and $y \in W^{\perp}$,

$$
\int_{\mathbf{S}_{W}}\left\langle y, \mathbf{w}_{i}(z)\right\rangle\langle x, z\rangle d \mathscr{H}^{m-1}(z)=0
$$

since $\mathcal{H}_{1} \perp \mathcal{H}_{i}$ if $i \neq 1$ and $z \mapsto\langle x, z\rangle \in \mathcal{H}_{1}$. Because $\sum_{i} \mathbf{w}_{i}$ converge to $\mathbf{w}$ in $L^{2}$ and (3.27) we get

$$
\left|\int_{\mathbf{S}_{W}}\left\langle y, \mathbf{w}_{1}(z)\right\rangle\langle x, z\rangle d \mathscr{H}^{m-1}(z)\right| \leq \mathbf{c}_{3.12^{\epsilon}},
$$

for all $x \in W$ and $y \in W^{\perp}$ with $|x|,|y| \leq 1$. Since $w_{1}^{l} p_{1}^{l}: W \rightarrow \mathbb{R}$ is a linear functional it is represented by some vector $w_{l} \in W$ with $\left\langle e_{l}, \mathbf{w}_{1}(z)\right\rangle=\left\langle w_{l}, z\right\rangle$. If we set $x=\sum_{k} \kappa^{k} e_{k}, y=\sum_{l} \lambda^{l} e_{l}$ and $w_{l}=\sum_{k^{\prime}} w_{l}^{k^{\prime}} e_{k^{\prime}} \in X$ it follows

$$
\begin{aligned}
\int_{\mathbf{S}_{W}}\left\langle y, \mathbf{w}_{1}(z)\right\rangle\langle x, z\rangle d \mathscr{H}^{m-1}(z) & =\sum_{k, l, l^{\prime}} \int_{\mathbf{S}_{W}}\left\langle\lambda^{l} e_{l},\left\langle w_{l^{\prime}}, z\right\rangle e_{l^{\prime}}\right\rangle\left\langle\kappa^{k} e_{k}, z\right\rangle d \mathscr{H}^{m-1}(z) \\
& =\sum_{k, l} \int_{\mathbf{S}_{W}} \kappa^{k} \lambda^{l}\left\langle w_{l}, z\right\rangle\left\langle e_{k}, z\right\rangle d \mathscr{H}^{m-1}(z) \\
& =\sum_{k, k^{\prime}, l} \int_{\mathbf{S}_{W}} \kappa^{k} \lambda^{l} w_{l}^{k^{\prime}}\left\langle e_{k^{\prime}}, z\right\rangle\left\langle e_{k}, z\right\rangle d \mathscr{H}^{m-1}(z) \\
& =\sum_{k, l} \int_{\mathbf{S}_{W}} \kappa^{k} \lambda^{l} w_{l}^{k}\left\langle e_{k}, z\right\rangle^{2} d \mathscr{H}^{m-1}(z) \\
& =\sum_{k, l} \mathbf{c}(m) \kappa^{k} \lambda^{l} w_{l}^{k}=\mathbf{c}(m) \sum_{k, l, l^{\prime}}\left\langle\lambda^{l} e_{l}, \kappa^{k} w_{l^{\prime}}^{k} e_{l^{\prime}}\right\rangle \\
& =\mathbf{c}(m) \sum_{l, l^{\prime}}\left\langle\lambda^{l} e_{l},\left\langle w_{l^{\prime}}, x\right\rangle e_{l^{\prime}}\right\rangle=\mathbf{c}(m)\left\langle y, \mathbf{w}_{1}(x)\right\rangle
\end{aligned}
$$


for some $\mathbf{c}(m)>0$ depending only on $m$. Hence letting $y=\mathbf{w}_{1}(x)$ and referring to (3.27),

$$
\left|\mathbf{w}_{1}(x)\right|=\left\langle\frac{\mathbf{w}_{1}(x)}{\left|\mathbf{w}_{1}(x)\right|}, \mathbf{w}_{1}(x)\right\rangle \leq \mathbf{c}(m)^{-1} \mathbf{c}_{3.12^{\epsilon}} .
$$

Since $x$ is arbitrary the proof is complete.

For $p, q \in \mathcal{H}_{k}\left(\mathbf{S}^{m-1}\right)$ it is a standard exercise using the divergence theorem to show that (with $\nabla$ we understand the gradient in $\mathbb{R}^{m}$ )

$$
\int_{\mathbf{S}^{m-1}} \nabla p \cdot \nabla q d \mathscr{H}^{m-1}=k(m+2 k-2) \int_{\mathbf{S}^{m-1}} p q d \mathscr{H}^{m-1},
$$

whereas for harmonic polynomials $p$ and $q$ of different degree both integrals are zero. With $\partial_{r} p=k p$ we get (for $|x|=1$ )

$$
\nabla p \cdot \nabla p=\|D p\|_{\mathrm{HS}}^{2}=k^{2} p^{2}+\left\|D_{S} p\right\|_{\mathrm{HS}}^{2},
$$

and hence

$$
\begin{aligned}
\int_{\mathbf{S}^{m-1}}\left\|D_{S} p\right\|_{\mathrm{HS}}^{2} d \mathscr{H}^{m-1} & =k(m+2 k-2) \int_{\mathbf{S}^{m-1}} p^{2} d \mathscr{H}^{m-1}-k^{2} \int_{\mathbf{S}^{m-1}} p^{2} d \mathscr{H}^{m-1} \\
& =k(m+k-2) \int_{\mathbf{S}^{m-1}} p^{2} d \mathscr{H}^{m-1} .
\end{aligned}
$$

More generally, if $f=\sum_{k} f_{k} p_{k}$ is a finite sum of homogeneous harmonic polynomials $p_{k}$ of degree $k$ and $L^{2}$-norm 1 we get as in [17]

$$
\int_{\mathbf{S}^{m-1}}\left\|D_{S} f\right\|_{\mathrm{HS}}^{2} d \mathscr{H}^{m-1}=\sum_{k} f_{k}^{2} k(m+k-2) .
$$

The restriction of any polynomial to the sphere is the finite sum of homogeneous harmonic polynomials and the formula remains true for $f$ in $C^{1}\left(\mathbf{S}^{m-1}, \mathbb{R}\right)$ by approximation.

Let $\mathbf{w}=\mathbf{w}_{0}+\mathbf{w}_{1}+\sum_{k \geq 2} \mathbf{w}_{k}$ as before with convergence in $L^{2}\left(\mathbf{S}^{m-1}, W^{\perp}\right)$, whereas with uniform convergence on $\mathbf{S}^{m-1}$,

$$
\mathbf{w}_{k}=\sum_{l>n} w_{k}^{l} p_{k}^{l} e_{l} .
$$

Note that $p_{0}^{l}=\mathscr{H}^{m-1}\left(\mathbf{S}^{m-1}\right)^{-\frac{1}{2}}=(\boldsymbol{\alpha}(m) m)^{-\frac{1}{2}}$ by normalization. As in [17], although there without the error term in $\epsilon$, we get the following estimate.

3.15. Lemma. There is some constant $\mathbf{c}_{3.15}(m)>0$ such that if $0<\epsilon<\epsilon_{3.12}$, then

$$
\int_{B_{W}(0,1)}\|D \mathbf{h}\|_{\mathrm{HS}}^{2} \leq \mathbf{c}_{3.15} \epsilon^{2}+\frac{2 m}{2 m+1} \int_{B_{W}(0,1)}\|D \mathbf{w}\|_{\mathrm{HS}}^{2} .
$$

Proof. With Lemma 3.13 and (3.29) we get

$$
\begin{aligned}
\int_{\mathbf{B}_{W}(0,1)}\|D \mathbf{h}\|_{\mathrm{HS}}^{2} & =\frac{1}{m+2} \int_{\mathbf{S}_{W}} 4\left|\mathbf{w}(x)-\mathbf{w}_{0}\right|^{2}+\left\|D_{S} \mathbf{w}_{x}\right\|_{\mathrm{HS}}^{2} d \mathscr{H}^{m-1}(x) \\
& =\frac{1}{m+2}\left[4 \sum_{l>m, k \geq 1}\left(w_{k}^{l}\right)^{2}+\sum_{l>m, k \geq 1}\left(w_{k}^{l}\right)^{2} k(m+k-2)\right] .
\end{aligned}
$$

By Lemma 3.14 it is

$$
\sum_{l>m}\left(w_{1}^{l}\right)^{2}=\left\|\mathbf{w}_{1}\right\|_{L^{2}}^{2} \leq \mathbf{c}^{\prime}\left\|\mathbf{w}_{1}\right\|_{\infty}^{2} \leq \mathbf{c} \epsilon^{2}
$$


for some constants $\mathbf{c}^{\prime}(m)>0$ and $\mathbf{c}(m)=\mathbf{c}_{3.14}(m) \mathbf{c}^{\prime}(m)>0$. Hence,

$$
\int_{\mathbf{B}_{W}(0,1)}\|D \mathbf{h}\|_{\mathrm{HS}}^{2} \leq \mathbf{c} \epsilon^{2}+\frac{1}{m+2} \sum_{l>m, k \geq 2}\left(w_{k}^{l}\right)^{2}[4+k(m+k-2)] .
$$

Similarly by Lemma 3.13,

$$
\int_{\mathbf{B}_{W}(0,1)}\|D \mathbf{w}\|_{\mathrm{HS}}^{2}=\frac{1}{m} \sum_{l>n, k \geq 0}\left(w_{k}^{l}\right)^{2}[1+k(m+k-2)] .
$$

As in [17],

$$
\begin{aligned}
\int_{\mathbf{B}_{W}(0,1)}\|D \mathbf{h}\|_{\mathrm{HS}}^{2} \leq \mathbf{c} \epsilon^{2}+\frac{1}{m+2} \sum_{l>m, k \geq 2}\left(w_{k}^{l}\right)^{2}[4+k(m+k-2)] \\
=\mathbf{c} \epsilon^{2}+\frac{1}{m+2} \sum_{l>m, k \geq 2}\left(w_{k}^{l}\right)^{2}[1+k(m+k-2)]\left[1+\frac{3}{1+k(m+k-2)}\right] \\
\leq \mathbf{c} \epsilon^{2}+\frac{m}{m+2}\left[1+\frac{3}{1+2 m}\right] \int_{\mathbf{B}_{W}(0,1)}\|D \mathbf{w}\|_{\mathrm{HS}}^{2} \\
=\mathbf{c} \epsilon^{2}+\frac{2 m}{2 m+1} \int_{\mathbf{B}_{W}(0,1)}\|D \mathbf{w}\|_{\mathrm{HS}}^{2} .
\end{aligned}
$$

3.4. A competitor better than the cone. In Subsection 3.2 we defined the comparison surface $T$ and in Proposition 3.11 we saw that $\mathbf{M}(T)$ can't be much bigger than $\mathbf{M}(P)$. We now modify $T$ on the cylinder $Z_{V}\left(\frac{1}{4}\right)$ to get a new chain $S$. We work on a 4 -times smaller scale than in the last subsection, so we replace the $\mathbf{h}$ constructed there by $\mathbf{h}_{4}:=4^{-1} \mathbf{h}(4 x)$. The new chain $S$ is defined by

(1) $S\left\llcorner Z_{W}\left(\frac{1}{4}\right)^{c}:=T\left\llcorner Z_{W}\left(\frac{1}{4}\right)^{c}\right.\right.$,

(2) $S\left\llcorner Z_{W}\left(\frac{1}{4}\right):=\left(\operatorname{id}_{W}+\mathbf{h}_{4}\right)_{\#}\left(g_{0} \llbracket \mathbf{B}_{W}\left(0, \frac{1}{4}\right) \rrbracket\right)\right.$.

The plane $W$ is close to $V$ by Lemma 3.12. So we can assume that $\epsilon$ is small enough such that

$$
\begin{gathered}
\operatorname{spt}(T) \cap Z_{W}\left(4^{-1}\right) \subset \operatorname{spt}(T) \cap Z_{V}\left(2^{-1}\right), \quad \text { and } \\
S\left\llcorner\mathbf{B}(0,1)^{c}=T\left\llcorner\mathbf{B}(0,1)^{c}=P\left\llcorner\mathbf{B}(0,1)^{c} .\right.\right.\right.
\end{gathered}
$$

By the construction of $\mathbf{h}$ and because $\mathbf{w}$ is 1-homogeneous we have $\mathbf{h}_{4}(x)=\mathbf{w}(x)$ for $|x|=\frac{1}{4}$ and hence $\partial P=\partial T=\partial S$. Note that the comparison surface $S$ is obtained via a push-forward from $T$ and hence also from $P$, i.e. $S=\psi_{\#} P$ for some Lipschitz map $\psi: X \rightarrow X$ with $\operatorname{spt}(\psi)$ contained in a neighborhood of 0 (for example, $\mathbf{B}_{V}(0,1)+\mathbf{B}_{V^{\perp}}(0,2 \rho)$ would be okay).

Before we give the main result, we compare the mass over the planes $W$ and $V$. The Lemma is essentially contained in [20].

3.16. Lemma ([20, Lemma 14]). There are constants $\mathbf{c}_{3.16}(m)>0$ and $0<\epsilon_{3.16} \leq$ $\epsilon_{3.12}$ such that if $0<\rho, \epsilon<\epsilon_{3.16}$, then

$$
\mathbf{M}\left(T_{\mathbf{v}}\left\llcorner Z_{W}\right) \leq \mathbf{M}\left(T_{\mathbf{v}}\left\llcorner Z_{V}\right)\right.\right.
$$

implies

$$
\mid \mathbf{M}\left(P\left\llcorner Z_{W}\right)-\mathbf{M}\left(P\left\llcorner Z_{V}\right)-\left[\mathbf { M } \left(T_{\mathbf{v}}\left\llcorner Z_{W}\right)-\mathbf{M}\left(T_{\mathbf{v}}\left\llcorner Z_{V}\right)\right] \mid \leq \mathbf{c}_{3.16}\left\|g_{0}\right\| \rho \epsilon .\right.\right.\right.\right.
$$


Proof. As noted in [20, Lemma 13], an elementary calculation shows that

$$
\begin{aligned}
& \mathbf{M}\left(T_{\mathbf{v}}\left\llcorner Z_{W}\right)=\int_{\mathbf{B}_{V}}\left\|g_{0}\right\|\left(1+\left(\mathcal{J} \mathbf{v}_{x}\right)^{2}\right)^{\frac{1}{2}}\left(\frac{|x|^{2}+|\mathbf{v}(x)|^{2}-k(x)^{2}}{|x|^{2}}\right)^{-\frac{m}{2}} d x,\right. \\
& \mathbf{M}\left(P\left\llcorner Z_{W}\right)=\int_{\mathbf{B}_{V}} \sum_{i \in I_{x}}\left\|g_{i}\right\|\left(1+\left(\mathcal{J} \mathbf{y}_{x}^{i}\right)^{2}\right)^{\frac{1}{2}}\left(\frac{|x|^{2}+\left|\mathbf{y}^{i}(x)\right|^{2}-k^{i}(x)^{2}}{|x|^{2}}\right)^{-\frac{m}{2}} d x,\right.
\end{aligned}
$$

where $k(x):=\left|\pi_{W^{\perp}}(x+\mathbf{v}(x))\right|$ and $k^{i}(x):=\left|\pi_{W^{\perp}}\left(x+\mathbf{y}^{i}(x)\right)\right|$. It is $\left|\mathbf{y}^{i}(x)\right| \leq \rho$ for $|x| \leq 1$ by the assumption on $P$. Using $\epsilon \leq \rho^{3}$,

$$
\begin{aligned}
k^{i}(x) & =\left|x+\mathbf{y}^{i}(x)-\pi_{W}\left(x+\mathbf{y}^{i}(x)\right)\right| \leq 2 \rho+\left|x-\pi_{W}(x)\right| \leq 2 \rho+\mathbf{c}_{3.12^{\epsilon^{\frac{1}{3}}}} \\
& \leq\left(2+\mathbf{c}_{3.12}\right) \rho .
\end{aligned}
$$

Similarly, $k(x) \leq\left(2 \mathbf{c}_{3.7}+\mathbf{c}_{3.12}\right) \epsilon^{\frac{1}{3}}$. For $i \in I_{x}$ let

$$
\begin{aligned}
\delta(x) & :=\left(\frac{|x|^{2}+|\mathbf{v}(x)|^{2}-k(x)^{2}}{|x|^{2}}\right)^{-\frac{m}{2}}-1, \\
\delta^{i}(x) & :=\left(\frac{|x|^{2}+\left|\mathbf{y}^{i}(x)\right|^{2}-k^{i}(x)^{2}}{|x|^{2}}\right)^{-\frac{m}{2}}-1 .
\end{aligned}
$$

The Taylor polynomial approximation for $t \in\left[-\frac{1}{2}, \frac{1}{2}\right]$ implies that $(1+t)^{-\frac{m}{2}}=$ $1-\frac{m}{2} t+R(t)$ and $R(t)=\frac{m(m+2)}{8}(1+\xi)^{-\frac{m+2}{2}} t^{2}$ for some $\xi \in\left[-\frac{1}{2}, \frac{1}{2}\right]$. So, if $\epsilon$ and $\rho$ are small, there is a constant $\mathbf{c}(m)>0$ such that

$$
\begin{aligned}
\left|\delta(x)-\frac{m}{2} \frac{k(x)^{2}-|\mathbf{v}(x)|^{2}}{|x|^{2}}\right| & \leq \mathbf{c} \epsilon^{\frac{4}{3}}, \\
\left|\delta^{i}(x)-\frac{m}{2} \frac{k^{i}(x)^{2}-\left|\mathbf{y}^{i}(x)\right|^{2}}{|x|^{2}}\right| & \leq \mathbf{c} \rho^{4} .
\end{aligned}
$$

Hence, for some $\mathbf{c}^{\prime}(m)>0$, both $\left|\delta^{i}(x)\right|$ and $|\delta(x)|$ are bounded by $\mathbf{c}^{\prime} \rho^{2}$. It is

$$
\begin{aligned}
\mathbf{M}\left(P\left\llcorner Z_{W}\right)-\mathbf{M}\left(P\left\llcorner Z_{V}\right)=\right.\right. & \int_{\mathbf{B}_{V}} \sum_{i \in I_{x}}\left\|g_{i}\right\|\left(1+\left(\mathcal{J} \mathbf{y}_{x}^{i}\right)^{2}\right)^{\frac{1}{2}} \delta^{i}(x) d x \\
= & \int_{\mathbf{B}_{V}} \sum_{i \in I_{x}}\left\|g_{i}\right\| \delta^{i}(x) d x \\
& +\int_{\mathbf{B}_{V}} \sum_{i \in I_{x}}\left\|g_{i}\right\| \delta^{i}(x)\left(\left(1+\left(\mathcal{J} \mathbf{y}_{x}^{i}\right)^{2}\right)^{\frac{1}{2}}-1\right) d x,
\end{aligned}
$$

and therefore

$$
\mid \mathbf{M}\left(P\left\llcorner Z_{W}\right)-\mathbf{M}\left(P\left\llcorner Z_{V}\right)-\int_{\mathbf{B}_{V}} \sum_{i \in I_{x}}\left\|g_{i}\right\| \delta^{i}(x) d x \mid \leq \mathbf{c}^{\prime}\left\|g_{0}\right\| \rho^{2} \epsilon,\right.\right.
$$


by the excess estimate on $P$ and the bound on $\left|\delta^{i}(x)\right|$ above. Similarly it follows from Lemma 3.8 and Lemma 3.10,

$$
\begin{aligned}
& \mid \mathbf{M}\left(T_{\mathbf{v}}\left\llcorner Z_{W}\right)-\mathbf{M}\left(T_{\mathbf{v}}\left\llcorner Z_{V}\right)-\int_{\mathbf{B}_{V}}\left\|g_{0}\right\| \delta(x) d x \mid\right.\right. \\
& \quad \leq \int_{\mathbf{B}_{V}}|\delta(x)|\left\|g_{0}\right\|\left(\left(1+\left(\mathcal{J} \mathbf{v}_{x}\right)^{2}\right)^{\frac{1}{2}}-1\right) d x \\
& \quad \leq \mathbf{c}^{\prime} \rho^{2}\left(\left(\mathbf{c}_{3.8}+\mathbf{c}_{3.10}\right)\left\|g_{0}\right\| \epsilon+\int_{\mathbf{B}_{V}} \sum_{i \in I_{x}}\left\|g_{i}\right\|\left(1+\left(\mathcal{J} \mathbf{y}_{x}^{i}\right)^{2}\right)^{\frac{1}{2}}-\left\|g_{0}\right\| d x\right) \\
& \quad \leq \mathbf{c}^{\prime \prime}\left\|g_{0}\right\| \rho^{2} \epsilon,
\end{aligned}
$$

for some constant $\mathbf{c}^{\prime \prime}(m)>0$. Since we assume that $\mathbf{M}\left(T_{\mathbf{v}}\left\llcorner Z_{W}\right) \leq \mathbf{M}\left(T_{\mathbf{v}}\left\llcorner Z_{V}\right)\right.\right.$ it follows from (3.35) that $\int_{\mathbf{B}_{V}}\left\|g_{0}\right\| \delta(x) d x \leq \mathbf{c}^{\prime \prime}\left\|g_{0}\right\| \rho^{2} \epsilon$. Then by (3.32),

$$
\begin{aligned}
\left\|g_{0}\right\| \int_{\mathbf{B}_{V}} \frac{m}{2} \frac{k(x)^{2}-|\mathbf{v}(x)|^{2}}{|x|^{2}} d x & \leq\left\|g_{0}\right\| \int_{\mathbf{B}_{V}} \delta(x)+\mathbf{c} \epsilon^{\frac{4}{3}} d x \\
& \leq\left(\mathbf{c}^{\prime \prime}+\boldsymbol{\alpha}(m) \mathbf{c}\right)\left\|g_{0}\right\| \rho^{2} \epsilon .
\end{aligned}
$$

By 1-homogeneity of $k$ and $\mathbf{v}$,

$$
\begin{aligned}
\left\|g_{0}\right\| \int_{\mathbf{B}_{V}} k(x)^{2}-|\mathbf{v}(x)|^{2} d x & =\left\|g_{0}\right\| \frac{m}{m+2} \int_{\mathbf{B}_{V}} \frac{k(x)^{2}-|\mathbf{v}(x)|^{2}}{|x|^{2}} d x \\
& \leq \frac{2}{m}\left(\mathbf{c}^{\prime \prime}+\boldsymbol{\alpha}(m) \mathbf{c}\right)\left\|g_{0}\right\| \rho^{2} \epsilon \\
& \leq \mathbf{c}^{\prime \prime \prime}\left\|g_{0}\right\| \rho \epsilon,
\end{aligned}
$$

for some $\mathbf{c}^{\prime \prime \prime}(m)>0$. Because of (3.2), Lemma 3.7, Lemma 3.8 and the assumption on $P$ and assuming $\epsilon$ is small,

$$
\begin{aligned}
\left\|g_{0}\right\| \int_{\mathbf{B}_{V}} \sum_{i \in I_{x}}|\mathbf{v}(x)|^{2} d x \leq & \left\|g_{0}\right\| \int_{E_{2}} \# I_{x}|\mathbf{v}(x)|^{2} d x+\left\|g_{0}\right\| \int_{E_{1}}|\mathbf{v}(x)|^{2} d x \\
\leq & 10 \mathbf{c}_{3.7}\left\|g_{0}\right\| \epsilon^{\frac{5}{3}}+\left\|g_{0}\right\| \int_{\mathbf{B}_{V}}|\mathbf{v}(x)|^{2} d x \\
\leq & 10 \mathbf{c}_{3.7}\left\|g_{0}\right\| \epsilon+4\left\|g_{0}\right\| \int_{\mathbf{B}_{V}}\left(1+|\mathbf{v}(x)|^{2}\right)^{\frac{1}{2}}-1 d x \\
\leq & 10 \mathbf{c}_{3.7}\left\|g_{0}\right\| \epsilon+4\left\|g_{0}\right\| \int_{\mathbf{B}_{V}}\left(1+\left\|D \mathbf{v}_{x}\right\|_{\mathrm{HS}}^{2}\right)^{\frac{1}{2}}-1 d x \\
\leq & \left(10 \mathbf{c}_{3.7}+4 \mathbf{c}_{3.8}\right)\left\|g_{0}\right\| \epsilon \\
& +4 \int_{\mathbf{B}_{V}} \sum_{i \in I_{x}}\left\|g_{i}\right\|\left(1+\left(\mathcal{J} \mathbf{y}_{x}^{i}\right)^{2}\right)^{\frac{1}{2}}-\left\|g_{0}\right\| d x \\
\leq & \mathbf{c}^{\prime \prime \prime \prime \prime}\left\|g_{0}\right\| \epsilon,
\end{aligned}
$$

for some $\mathbf{c}^{\prime \prime \prime \prime}(m)>0$. Using a similar calculation, the bound $k(x) \leq\left(2 \mathbf{c}_{3.7}+\right.$ $\mathbf{c}_{3.12)} \epsilon^{\frac{1}{3}}$ and (3.36), $\mathbf{c}^{\prime \prime \prime \prime}$ can be assumed big enough such that also

$$
\left\|g_{0}\right\| \int_{\mathbf{B}_{V}} \sum_{i \in I_{x}} k(x)^{2} d x \leq \mathbf{c}^{\prime \prime \prime \prime}\left\|g_{0}\right\| \epsilon .
$$


There is a Lipschitz constant $L(m)>0$ for which $\left|(1+s)^{-\frac{m}{2}}-(1+t)^{-\frac{m}{2}}\right| \leq L|s-t|$ if $|s|,|t| \leq \frac{1}{2}$. The assumption on $P$ and the 1-homogeneity of $\mathbf{v}, \mathbf{y}^{i}, k$ and $k^{i}$ imply with (3.2), (3.3), (3.32) and (3.33),

$$
\begin{aligned}
\int_{\mathbf{B}_{V}} & \left|\left\|g_{0}\right\| \delta(x)-\sum_{i \in I_{x}}\left\|g_{i}\right\| \delta^{i}(x)\right| d x \\
= & \left\|g_{0}\right\| \int_{\mathbf{B}_{V}}\left|\left(1-\# I_{x}\right) \delta(x)\right| d x+\int_{\mathbf{B}_{V}}\left|\sum_{i \in I_{x}}\left\|g_{0}\right\| \delta^{i}(x)-\left\|g_{i}\right\| \delta^{i}(x)\right| d x \\
& +\left\|g_{0}\right\| \int_{\mathbf{B}_{V}} \sum_{i \in I_{x}}\left|\delta(x)-\delta^{i}(x)\right| d x \\
\leq & \mathbf{c}^{\prime} \rho^{2}\left\|g_{0}\right\| \int_{E_{2}} \# I_{x} d x+\mathbf{c}^{\prime} \rho^{2} \int_{E_{2}} \# I_{x}\left\|g_{0}\right\|+\sum_{i \in I_{x}}\left\|g_{i}\right\| d x \\
& +L\left\|g_{0}\right\| \int_{\mathbf{B}_{V}} \sum_{i \in I_{x}}\left|\frac{k(x)^{2}-|\mathbf{v}(x)|^{2}}{|x|^{2}}-\frac{k^{i}(x)^{2}-\left|\mathbf{y}^{i}(x)\right|^{2}}{|x|^{2}}\right| d x \\
\leq & 35 \mathbf{c}^{\prime}\left\|g_{0}\right\| \rho^{2} \epsilon+\left.3 L\left\|g_{0}\right\| \int_{\mathbf{B}_{V}} \sum_{i \in I_{x}}|| \mathbf{v}(x)\right|^{2}-\left|\mathbf{y}^{i}(x)\right|^{2}|+| k(x)^{2}-k^{i}(x)^{2} \mid d x .
\end{aligned}
$$

Instead of the factor 3 above we could use $\frac{m+2}{m}$. Since $\left|k(x)-k^{i}(x)\right| \leq\left|\mathbf{v}(x)-\mathbf{y}^{i}(x)\right|$ by the definition of $k$ and $k^{i}$, there holds

$$
\begin{aligned}
\left.|| \mathbf{v}(x)\right|^{2}-\left|\mathbf{y}^{i}(x)\right|^{2} \mid+ & \left|k(x)^{2}-k^{i}(x)^{2}\right| \\
\leq & \left|\mathbf{v}(x)-\mathbf{y}^{i}(x)\right|^{2}+2|\mathbf{v}(x)|\left|\mathbf{v}(x)-\mathbf{y}^{i}(x)\right| \\
& +\left|k(x)-k^{i}(x)\right|^{2}+2 k(x)\left|k(x)-k^{i}(x)\right| \\
\leq & 2\left|\mathbf{v}(x)-\mathbf{y}^{i}(x)\right|^{2}+2(|\mathbf{v}(x)|+k(x))\left|\mathbf{v}(x)-\mathbf{y}^{i}(x)\right| .
\end{aligned}
$$

Hence by Lemma 3.5, the fact that $2 a b \leq \rho a^{2}+\rho^{-1} b^{2},(3.37)$ and (3.38),

$$
\begin{aligned}
\mid \int_{\mathbf{B}_{V}} & \left\|g_{0}\right\| \delta(x)-\sum_{i \in I_{x}}\left\|g_{i}\right\| \delta^{i}(x) d x \mid \\
\leq & 35 \mathbf{c}^{\prime}\left\|g_{0}\right\| \rho^{2} \epsilon \\
& +3 L\left\|g_{0}\right\| \int_{\mathbf{B}_{V}} \sum_{i \in I_{x}} 2\left|\mathbf{v}(x) \| \mathbf{v}(x)-\mathbf{y}^{i}(x)\right|+2 k(x)\left|\mathbf{v}(x)-\mathbf{y}^{i}(x)\right| d x \\
\leq & 35 \mathbf{c}^{\prime}\left\|g_{0}\right\| \rho^{2} \epsilon+3 L\left\|g_{0}\right\| \int_{\mathbf{B}_{V}} \sum_{i \in I_{x}} \rho|\mathbf{v}(x)|^{2}+\rho k(x)^{2}+2 \rho^{-1}\left|\mathbf{v}(x)-\mathbf{y}^{i}(x)\right|^{2} d x \\
\leq & \left(35 \mathbf{c}^{\prime}+6 \mathbf{c}^{\prime \prime \prime \prime} L+6 \mathbf{c}_{3.5} L\right)\left\|g_{0}\right\| \rho \epsilon .
\end{aligned}
$$

If we apply this in (3.34) and (3.35), the lemma follows.

Next we give another technical lemma that will be used in the main result of this section. It essentially tells that if a rather flat cone is far away from some plane, then the cylindrical excess over this plane is large.

3.17. Lemma. Let $C \in \mathscr{P}_{m}(X ; G)$ be an infinite (or large enough) cone with center 0 . Assume that there is some $0<\delta<\frac{1}{5}$ and $V \in \mathbf{G}(X, m)$ such that,

(1) $\operatorname{spt}\left(\partial\left(C\left\llcorner Z_{V}(0,2)\right)\right) \subset X \backslash Z_{V}(0,2)\right.$, 
(2) $\pi_{V \#}\left(C\left\llcorner Z_{V}\right)=g_{0} \llbracket \boldsymbol{B}_{V}(0,1) \rrbracket\right.$,

(3) $\left|\pi_{V \perp}(x)\right| \leq \delta$ for $x \in \operatorname{spt}(C) \cap Z_{V}$.

If for some $\lambda \geq 2$ and $U \in \mathbf{G}(X, m)$ there holds

$$
\frac{1}{5}>\left\|\pi_{U}-\pi_{V}\right\|>6 \lambda \delta,
$$

then $\left|\pi_{U^{\perp}}(x)\right|>2(\lambda-2) \delta$ for some $x \in \operatorname{spt}(C) \cap Z_{U}$ and

$$
\operatorname{Exc}_{1}(C, U) \geq\left\|g_{0}\right\| \frac{\boldsymbol{\alpha}(m)}{3^{m+1}} \lambda^{2} \delta^{2}
$$

Proof. Note first that Lemma 2.7 implies $\pi_{U \#}\left(C\left\llcorner Z_{U}\right)=g_{0} \llbracket \mathbf{B}_{U}(0,1) \rrbracket\right.$ for the same group element $g_{0} \in G$. Let $A$ be the set of points $u \in \mathbf{B}_{U}(0,1)$ for which $\left|\pi_{V^{\perp}}(u)\right| \leq$ $2 \lambda \delta$. $A$ is closed, contains the origin and by the triangle inequality it is also convex. By assumption and Lemma 2.1 there is some $u \in \mathbf{B}_{U}(0,1)$ such that $\left|\pi_{V^{\perp}}(u)\right|=$ $\left|\pi_{V}(u)-u\right|>6 \lambda \delta$. Hence $\frac{1}{3} u \notin A$. By the hyperplane separation theorem there is a half-plane $U_{0}$ of $U$ that contains $\frac{1}{3} u$ and is disjoint from $A$. Hence there is some ball $A^{\prime}:=\mathbf{B}_{U}\left(u_{0}, \frac{1}{3}\right) \subset \mathbf{B}_{U}(0,1) \backslash A$. Let $x \in \operatorname{spt}(C) \cap Z_{U}$ with $\pi_{U}(x) \in A^{\prime}$ and $\left|\pi_{U^{\perp}}(x)\right| \leq 2 \lambda \delta$. Then $|x| \leq 1+2 \lambda \delta \leq 2$ and hence $\left|\pi_{V^{\perp}}(x)\right| \leq \operatorname{dist}(V, \operatorname{spt}(C) \cap$ $\left.Z_{V}(0,2)\right) \leq 2 \delta$. Further,

$$
\begin{aligned}
\left|\pi_{V^{\perp}}(x)\right|+\left|\pi_{U^{\perp}}(x)\right| & \geq\left|\pi_{V^{\perp}}(x)\right|+\left|\pi_{V^{\perp}}\left(\pi_{U^{\perp}}(x)\right)\right| \geq\left|\pi_{V^{\perp}}\left(x-\pi_{U^{\perp}}(x)\right)\right| \\
& \geq\left|\pi_{V^{\perp}}\left(\pi_{U}(x)\right)\right|>2 \lambda \delta,
\end{aligned}
$$

and because $\lambda \geq 2$,

$$
\left|\pi_{U^{\perp}}(x)\right|>2 \lambda \delta-2 \delta \geq \lambda \delta .
$$

This shows the first conclusion and further that if $x \in \operatorname{spt}(C)$ with $\pi_{U}(x) \in A^{\prime}$, then $\left|\pi_{U^{\perp}}(x)\right| \geq 2 \delta$.

Set $C^{\prime}=\sum_{i} g_{i} \llbracket S_{i} \rrbracket$ as a finite sum over oriented simplices $S_{i}$ for which one vertex is at the origin and the other vertices outside $Z_{U}$ such that $C\left\llcorner Z_{U}=C^{\prime}\left\llcorner Z_{U}\right.\right.$. Let $A^{\prime \prime}$ be the subset of those points $u \in A^{\prime}$ for which the set $\pi_{U}{ }^{-1}\{u\} \cap \operatorname{spt}\left(C^{\prime}\right)$ is finite and contained in the interior of any simplex it intersects. $\pi_{U}\left(A^{\prime \prime}\right)$ has full measure in $A^{\prime}$. For $x \in A^{\prime \prime}$ let $I_{x}$ be the collection of those $i$ for which $x \in \pi_{U}\left(S_{i}\right)$ and as we did for $P$ in the first sections, let $\mathbf{y}^{i}: U \rightarrow U^{\perp}$ be the affine map such that $x^{\prime}+\mathbf{y}^{i}\left(x^{\prime}\right)$ is in $S_{i}$ if $x^{\prime}$ is close to $x$. From Lemma 2.9 it follows that $\sum_{i \in I_{x}}\left\|g_{i}\right\| \geq\left\|g_{0}\right\|$. It is $\mathcal{J} \mathbf{y}_{x}^{i} \geq\left\|D \mathbf{y}_{x}^{i}\right\| \geq\left|\mathbf{y}^{i}(x)\right| \geq 2 \delta$ for $x \in A^{\prime \prime}$ by (2.5) and (2.6). Using $\left(1+a^{2}\right)^{\frac{1}{2}} \geq 1+\frac{1}{3} a^{2}$ for $a \leq 1$ we get

$$
\begin{aligned}
\operatorname{Exc}_{1}(C, U) & \geq \operatorname{Exc}\left(C, U, A^{\prime \prime}\right)=\int_{A^{\prime \prime}} \sum_{i \in I_{x}}\left\|g_{i}\right\|\left(1+\left(\mathcal{J} \mathbf{y}_{x}^{i}\right)^{2}\right)^{\frac{1}{2}}-\left\|g_{0}\right\| d x \\
& \geq \int_{A^{\prime \prime}} \sum_{i \in I_{x}}\left\|g_{i}\right\|\left(1+(\lambda \delta)^{2}\right)^{\frac{1}{2}}-\left\|g_{0}\right\| d x \\
& \geq\left\|g_{0}\right\| \mathscr{H}^{m}\left(A^{\prime \prime}\right)\left[\left(1+(\lambda \delta)^{2}\right)^{\frac{1}{2}}-1\right] \\
& \geq\left\|g_{0}\right\| \frac{\boldsymbol{\alpha}(m)}{3^{m+1}} \lambda^{2} \delta^{2} .
\end{aligned}
$$

Below is the main result of this section, the epiperimetric inequality of Reifenberg [20] adapted to $G$-chains in Hilbert spaces. 
3.18. Theorem. There is a constant $\epsilon_{3.18}(m)>0$ with the following property. Let $P \in \mathscr{P}_{m}(X ; G)$ be a cone with center 0 and $V$ be an $m$-plane such that:

(1) $\operatorname{spt}(\partial P) \cap Z_{V}(2)=\emptyset$,

(2) $\pi_{V \#}\left(P\left\llcorner Z_{V}\right)=g_{0} \llbracket \boldsymbol{B}_{V}(0,1) \rrbracket\right.$ for some $g_{0} \in G \backslash\left\{0_{G}\right\}$,

(3) $\|\mathbf{g}(x)\| \geq \frac{3}{4}\left\|g_{0}\right\|$ for $\|P\|$-almost every $x \in X$

(4) $\left|\pi_{V^{\perp}}(x)\right|<\epsilon_{3.18}$ for $x \in \operatorname{spt}(P) \cap Z_{V}$,

(5) $\operatorname{Exc}_{1}(P, V)<\left\|g_{0}\right\| \epsilon_{3.18}$.

Then there is some $S \in \mathscr{R}_{m}(X ; G)$ with $\partial(S\llcorner\boldsymbol{B}(0,1))=\partial(P\llcorner\boldsymbol{B}(0,1))$ and

$$
\mathbf{M}\left(S\llcorner\boldsymbol{B}(0,1))-\left\|g_{0}\right\| \boldsymbol{\alpha}(m) \leq \lambda_{3.18}\left(\mathbf{M}\left(P\llcorner\boldsymbol{B}(0,1))-\left\|g_{0}\right\| \boldsymbol{\alpha}(m)\right)\right.\right.
$$

for $\lambda_{3.18}:=\frac{2 m+1-4^{-m-1}}{2 m+1}<1$.

Proof. As noted in the beginning of this section, the group norm can be normalized such that $\left\|g_{0}\right\|=1$. Let $\epsilon_{3.18}>0$ be small enough such that

$$
24 \lambda \sqrt{\epsilon_{3.18}}<\left(2 \epsilon_{3.18}\right)^{\frac{1}{6 m}} \leq \epsilon_{3.12}<\frac{1}{20} .
$$

where $\lambda^{2}:=2 \boldsymbol{\alpha}(m)^{-1} 3^{m+1} \geq \frac{3^{m}}{2^{m}} 6 \geq 2^{2}$. Let $V^{\prime} \in \mathbf{G}(X, m)$ be such that

$$
\operatorname{Exc}_{1}\left(P, V^{\prime}\right)<2 \inf \left\{\operatorname{Exc}_{1}(P, U):\left\|\pi_{V}-\pi_{U}\right\|<\frac{1}{10}\right\} .
$$

Then $\epsilon:=\operatorname{Exc}_{1}\left(P, V^{\prime}\right)<2 \epsilon_{3.18} \leq \epsilon_{3.12}$ and hence $\left\|\pi_{V}-\pi_{V^{\prime}}\right\| \leq 6 \lambda \sqrt{\epsilon_{3.18}}<\frac{1}{20}$ by Lemma 3.17. Since $\operatorname{spt}(P) \cap Z_{V^{\prime}} \subset \mathbf{B}(0,2)$, there holds $\left|\pi_{V^{\prime \perp}}(x)\right| \leq 24 \lambda \sqrt{\epsilon_{3.18}}<$ $\rho:=\left(2 \epsilon_{3.18}\right)^{\frac{1}{6 m}} \leq \epsilon_{3.12}$. In particular

$$
\operatorname{Exc}_{1}\left(P, V^{\prime}\right)<2 \inf \left\{\operatorname{Exc}_{1}(P, U):\left\|\pi_{V^{\prime}}-\pi_{U}\right\|<\frac{1}{20}\right\},
$$

and Lemma 3.12 implies $\left\|\pi_{V^{\prime}}-\pi_{W}\right\|<\frac{1}{20}$ for the plane $W$ used in the construction of the competitor $S$. By changing $V^{\prime}$ slightly we can assume that $P$ is in general position with respect to $V^{\prime}\left(\operatorname{Exc}_{1}(P, U)\right.$ is continuous in $U$ and the $m$-planes for which $P$ is in general position are dense in $\mathbf{G}(X, m))$. Hence $\epsilon, \rho$ and $V^{\prime}$ satisfy all the conditions at the beginning of Subsection 3.1 and the subsequent estimates. For the rest of this proof we identify $V^{\prime}$ with $V$. Let $S$ be the comparison surface as described in the beginning of this subsection. Lemma 3.13 and Lemma 3.15 imply

$$
\begin{aligned}
\mathbf{M}\left(S\left\llcorner Z_{W}\left(4^{-1}\right)\right)-4^{-m} \boldsymbol{\alpha}(m)=\right. & 4^{-m} \int_{\mathbf{B}_{W}}\left(1+\left(\mathcal{J} \mathbf{h}_{x}\right)^{2}\right)^{\frac{1}{2}}-1 d x \\
\leq & \mathbf{c}_{3.13^{\epsilon^{\frac{4}{3}}}+4^{-m}} \int_{\mathbf{B}_{W}}\left(1+\left\|D \mathbf{h}_{x}\right\|_{\mathrm{HS}}^{2}\right)^{\frac{1}{2}}-1 d x \\
\leq & \mathbf{c}_{3.13^{\epsilon^{\frac{4}{3}}}+4^{-m}} \int_{\mathbf{B}_{W}} \frac{1}{2}\left\|D \mathbf{h}_{x}\right\|_{\mathrm{HS}}^{2} d x \\
\leq & \left(\mathbf{c}_{3.13}+\mathbf{c}_{3.15}\right) \epsilon^{\frac{4}{3}} \\
& +4^{-m} \frac{2 m}{2 m+1} \int_{\mathbf{B}_{W}} \frac{1}{2}\left\|D \mathbf{w}_{x}\right\|_{\mathrm{HS}}^{2} d x .
\end{aligned}
$$




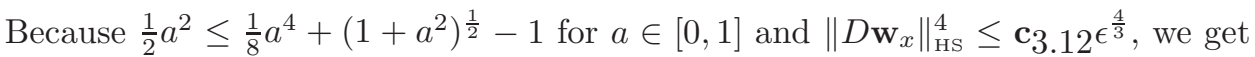
for some $\mathbf{c}(m)>0$,

$$
\begin{aligned}
\mathbf{M}\left(S\left\llcorner Z_{W}\left(4^{-1}\right)\right)-4^{-m} \boldsymbol{\alpha}(m)\right. \\
\quad \leq \mathbf{c} \epsilon^{\frac{4}{3}}+4^{-m} \frac{2 m}{2 m+1} \int_{\mathbf{B}_{W}}\left(1+\left\|D \mathbf{w}_{x}\right\|_{\mathrm{HS}}^{2}\right)^{\frac{1}{2}}-1 d x \\
\leq \mathbf{c} \epsilon^{\frac{4}{3}}+4^{-m} \frac{2 m}{2 m+1} \int_{\mathbf{B}_{W}}\left(1+\left(\mathcal{J} \mathbf{w}_{x}\right)^{2}\right)^{\frac{1}{2}}-1 d x \\
\quad=\mathbf{c} \epsilon^{\frac{4}{3}}+\frac{2 m}{2 m+1}\left[\mathbf{M}\left(T\left\llcorner Z_{W}\left(4^{-1}\right)\right)-4^{-m} \boldsymbol{\alpha}(m)\right] .\right.
\end{aligned}
$$

Hence,

$$
\begin{aligned}
\mathbf{M}\left(S\left\llcorner Z_{V}\right)=\right. & \mathbf{M}\left(T\left\llcorner\left(Z_{V} \backslash Z_{W}\left(4^{-1}\right)\right)\right)+\mathbf{M}\left(S\left\llcorner Z_{W}\left(4^{-1}\right)\right)\right.\right. \\
\leq & \mathbf{c} \epsilon^{\frac{4}{3}}+\mathbf{M}\left(T\left\llcorner\left(Z_{V} \backslash Z_{W}\left(4^{-1}\right)\right)\right)\right. \\
& +\frac{2 m}{2 m+1} \mathbf{M}\left(T\left\llcorner Z_{W}\left(4^{-1}\right)\right)+\frac{4^{-m} \boldsymbol{\alpha}(m)}{2 m+1}\right. \\
= & \mathbf{c} \epsilon^{\frac{4}{3}}+\mathbf{M}\left(T\left\llcorner Z_{V}\right)-\frac{1}{2 m+1}\left[\mathbf{M}\left(T\left\llcorner Z_{W}\left(4^{-1}\right)\right)-4^{-m} \boldsymbol{\alpha}(m)\right] .\right.\right.
\end{aligned}
$$

If $\mathbf{M}\left(T\left\llcorner Z_{W}\left(4^{-1}\right)\right) \geq \mathbf{M}\left(T\left\llcorner Z_{V}\left(4^{-1}\right)\right)\right.\right.$ we estimate using Proposition 3.11,

$$
\begin{aligned}
\mathbf{M}\left(S\left\llcorner Z_{V}\right) \leq\right. & \mathbf{c} \epsilon^{\frac{4}{3}}+\mathbf{M}\left(T\left\llcorner Z_{V}\right)-\frac{1}{2 m+1}\left[\mathbf{M}\left(T\left\llcorner Z_{W}\left(4^{-1}\right)\right)-4^{-m} \boldsymbol{\alpha}(m)\right]\right.\right. \\
\leq & \mathbf{c} \epsilon^{\frac{4}{3}}+\mathbf{M}\left(T\left\llcorner\left(Z_{V} \backslash Z_{V}\left(4^{-1}\right)\right)\right)\right. \\
& +\frac{2 m}{2 m+1} \mathbf{M}\left(T\left\llcorner Z_{V}\left(4^{-1}\right)\right)+\frac{4^{-m} \boldsymbol{\alpha}(m)}{2 m+1}\right. \\
\leq & \left(\mathbf{c}+\mathbf{c}_{3.11}\right) \rho^{\frac{1}{2}} \epsilon+\mathbf{M}\left(P\left\llcorner\left(Z_{V} \backslash Z_{V}\left(4^{-1}\right)\right)\right)\right. \\
& +\frac{2 m}{2 m+1} \mathbf{M}\left(P\left\llcorner Z_{V}\left(4^{-1}\right)\right)+\frac{4^{-m} \boldsymbol{\alpha}(m)}{2 m+1}\right. \\
= & \left(\mathbf{c}+\mathbf{c}_{3.11}\right) \rho^{\frac{1}{2}} \epsilon+\mathbf{M}\left(P\left\llcorner Z_{V}\right)\right. \\
& -\frac{1}{2 m+1}\left[\mathbf{M}\left(P\left\llcorner Z_{V}\left(4^{-1}\right)\right)-4^{-m} \boldsymbol{\alpha}(m)\right]\right. \\
= & \left(\mathbf{c}+\mathbf{c}_{3.11}\right) \rho^{\frac{1}{2}} \epsilon+\frac{2 m+1-4^{-m}}{2 m+1} \mathbf{M}\left(P\left\llcorner Z_{V}\right)+\frac{4^{-m}}{2 m+1} \boldsymbol{\alpha}(m) .\right.
\end{aligned}
$$

On the other hand, if $\mathbf{M}\left(T\left\llcorner Z_{W}\left(4^{-1}\right)\right) \leq \mathbf{M}\left(T\left\llcorner Z_{V}\left(4^{-1}\right)\right)\right.\right.$ it follows from Lemma 3.16 and the almost minimality of the plane $V$ with respect to the excess of $P$,

$$
\begin{aligned}
\operatorname{Exc}_{4^{-1}}(T, V) & \leq \mathbf{c}_{3.16} \rho \epsilon+\operatorname{Exc}_{4^{-1}}(T, W)+\operatorname{Exc}_{4^{-1}}(P, V)-\operatorname{Exc}_{4^{-1}}(P, W) \\
& \leq \mathbf{c}_{3.16} \rho \epsilon+\operatorname{Exc}_{4^{-1}}(T, W)+2^{-1} \operatorname{Exc}_{4^{-1}}(P, V) .
\end{aligned}
$$


Hence, with the same derivations as in the other case above,

$$
\begin{aligned}
& \mathbf{M}\left(S\left\llcorner Z_{V}\right) \leq \mathbf{c} \epsilon^{\frac{4}{3}}+\right. \mathbf{M}\left(T\left\llcorner Z_{V}\right)-\frac{1}{2 m+1} \operatorname{Exc}_{4^{-1}}(T, W)\right. \\
& \leq\left(\mathbf{c}+\mathbf{c}_{3.16}\right) \rho \epsilon+\mathbf{M}\left(T\left\llcorner Z_{V}\right)\right. \\
& \quad-\frac{1}{2 m+1}\left[\operatorname{Exc}_{4^{-1}}(T, V)-2^{-1} \operatorname{Exc}_{4^{-1}}(P, V)\right] \\
& \leq\left(\mathbf{c}+\mathbf{c}_{3.11}+\mathbf{c}_{3.16}\right) \rho^{\frac{1}{2}} \epsilon+\frac{2 m+1-4^{-m}}{2 m+1} \mathbf{M}\left(P\left\llcorner Z_{V}\right)\right. \\
& \quad+\frac{4^{-m}}{2 m+1} \boldsymbol{\alpha}(m)+\frac{2^{-1} 4^{-m}}{2 m+1} \operatorname{Exc}_{1}(P, V) .
\end{aligned}
$$

By subtracting $\boldsymbol{\alpha}(m)$ from these estimates we obtain in both cases

$$
\operatorname{Exc}_{1}(S, V) \leq \mathbf{c}^{\prime} \rho^{\frac{1}{2}} \epsilon+\frac{2 m+1-2^{-1} 4^{-m}}{2 m+1} \operatorname{Exc}_{1}(P, V)
$$

for some $\mathbf{c}^{\prime}(m)>0$. If we assume that $\epsilon_{3.18}$ is small enough such that for $\rho=$ $\left(2 \epsilon_{3.18}\right)^{\frac{1}{6 m}}$,

$$
\mathbf{c}^{\prime} \rho^{\frac{1}{2}}+\frac{2 m+1-2^{-1} 4^{-m}}{2 m+1} \leq \frac{2 m+1-4^{-m-1}}{2 m+1}=\lambda,
$$

we obtain $\operatorname{Exc}_{1}(S, V) \leq \lambda \operatorname{Exc}_{1}(P, V)$. Finally, by (3.31),

$$
\begin{aligned}
\mathbf{M}(S\llcorner\mathbf{B}(0,1))-\boldsymbol{\alpha}(m)= & \mathbf{M}\left(S\left\llcorner Z_{V}\right)-\mathbf{M}\left(P\left\llcorner\left(Z_{V} \backslash \mathbf{B}(0,1)\right)\right)-\boldsymbol{\alpha}(m)\right.\right. \\
\leq & \lambda\left[\mathbf { M } \left(P\llcorner\mathbf{B}(0,1))+\mathbf{M}\left(P\left\llcorner\left(Z_{V} \backslash \mathbf{B}(0,1)\right)\right)-\boldsymbol{\alpha}(m)\right]\right.\right. \\
& -\mathbf{M}\left(P\left\llcorner\left(Z_{V} \backslash \mathbf{B}(0,1)\right)\right)\right. \\
\leq & \lambda(\mathbf{M}(P\llcorner\mathbf{B}(0,1))-\boldsymbol{\alpha}(m)) .
\end{aligned}
$$

\section{Moments computations and Good approximations By planes}

4.1. Nearly monotonic, almost monotonic and epiperimetric chains. Let $\phi$ be a finite Borel measure on $X$. For $x \in X$ and $r>0$ we define

$$
\begin{aligned}
& \operatorname{exc}_{*}^{m}(\phi, x, r):=\sup \left\{\left(\frac{\phi(\mathbf{B}(x, t))}{\boldsymbol{\alpha}(m) t^{m}}-\frac{\phi(\mathbf{B}(x, s))}{\boldsymbol{\alpha}(m) s^{m}}\right)_{-}: 0<s \leq t \leq r\right\}, \\
& \mathbf{e x c}^{m *}(\phi, x, r):=\sup \left\{\left(\frac{\phi(\mathbf{B}(x, t))}{\boldsymbol{\alpha}(m) t^{m}}-\frac{\phi(\mathbf{B}(x, s))}{\boldsymbol{\alpha}(m) s^{m}}\right)_{+}: 0<s \leq t \leq r\right\}, \\
& \mathbf{e x c}^{m}(\phi, x, r):=\max \left\{\mathbf{e x c}_{*}^{m}(\phi, x, r), \mathbf{e x c}^{m *}(\phi, x, r)\right\} .
\end{aligned}
$$

Throughout the rest of these notes we use the term gauge or gauge function for an increasing function $\xi:(0, \delta] \rightarrow \mathbb{R}_{+}$for some $\delta>0$ with $\lim _{r \rightarrow 0} \xi(r)=0$. Let $T \in \mathscr{R}_{m}(X ; G)$. For a subset $A \subset X \backslash \operatorname{spt}(\partial T)$ we say that $T$ is nearly monotonic in $A$ if there is a gauge $\xi_{*}:(0, \delta] \rightarrow \mathbb{R}_{+}$such that

$$
\mathbf{e x c}_{*}^{m}(\|T\|, x, r) \leq \xi_{*}(r)
$$

for all $x \in A$ and $0<r<\min \{\delta, \operatorname{dist}(x, \operatorname{spt}(\partial T))\}$. We say that $T$ is almost monotonic in $A$ if there is a gauge $\xi:(0, \delta] \rightarrow \mathbb{R}_{+}$such that

$$
r \mapsto \exp (\xi(r)) \frac{\|T\|(\mathbf{B}(x, r))}{\boldsymbol{\alpha}(m) r^{m}}
$$


is an increasing function for all $x \in A$ and all $0<r<\min \{\delta$, $\operatorname{dist}(x, \operatorname{spt}(\partial T))\}$.

The following Lemma is an adaptation of [11, Lemma 3.2.3] to the setting of rectifiable chains Hilbert spaces. The proof goes through unchanged.

4.1. Lemma. If $T \in \mathscr{R}_{m}(X ; G)$ is nearly monotonic in $A \subset X \backslash \operatorname{spt}(\partial T)$, then the density $\Theta^{m}(\|T\|, x)$ exists for all $x \in A$ and is finite. Further, the function $x \mapsto \Theta^{m}(\|T\|, x)$ is upper semicontinuous in $A$.

Proof. Let $\xi_{*}:(0, \delta] \rightarrow \mathbb{R}_{+}$be a gauge with respect to which $T$ is nearly monotonic. For $x \in A$ and $0<r<\min \{\delta$, $\operatorname{dist}(x, \operatorname{spt}(\partial T))\}$ we abbreviate $f_{x}(r):=\boldsymbol{\alpha}(m)^{-1} r^{-m}\|T\|(\mathbf{B}(x, r))$. By assumption

$$
-\xi_{*}(R) \leq f_{x}(R)-f_{x}(r),
$$

for all $x \in A$ and $0<r \leq R<\min \{\delta, \operatorname{dist}(x, \operatorname{spt}(\partial T))\}$. Therefore,

$$
-\xi_{*}(R) \leq \liminf _{r \downarrow 0}\left(f_{x}(R)-f_{x}(r)\right)=f_{x}(R)-\limsup _{r \downarrow 0} f_{x}(r),
$$

and, in turn,

$$
0 \leq \liminf _{R \downarrow 0} f_{x}(R)-\limsup _{r \downarrow 0} f_{x}(r) .
$$

This shows that the densities $\Theta^{m}(\|T\|, x)$ exist for all $x \in A$. Let $\left(x_{i}\right)$ be a sequence in $A$ with $\lim _{i \rightarrow \infty} x_{i}=x \in A$ and $\left|x_{i}-x\right|<2^{-1} \min \{\delta, \operatorname{dist}(x, \operatorname{spt}(\partial T))\}$ for all $i$. Since $\|T\|$ is a finite Borel measure, the function $\rho \mapsto\|T\|(\mathbf{B}(x, \rho))$ is continuous in all but countably many $r>0$. If we pick a point of continuity $0<r<2^{-1} \min \{\delta, \operatorname{dist}(x, \operatorname{spt}(\partial T))\}$, then

$$
\begin{aligned}
\Theta^{m}\left(\|T\|, x_{i}\right) & \leq f_{x_{i}}(r)+\xi_{*}(r) \\
& \leq f_{x}\left(r+\left|x_{i}-x\right|\right)\left(1+\frac{\left|x_{i}-x\right|}{r}\right)^{m}+\xi_{*}(r),
\end{aligned}
$$

for all $i$. Hence for all such $r$,

$$
\limsup _{i \rightarrow \infty} \Theta^{m}\left(\|T\|, x_{i}\right) \leq f_{x}(r)+\xi_{*}(r) .
$$

Taking the limit for $r \rightarrow 0$, we see that $\lim \sup _{i \rightarrow \infty} \Theta^{m}\left(\|T\|, x_{i}\right) \leq \Theta^{m}(\|T\|, x)$.

The following observation is essentially [11, Lemma 3.4.1]. For the reader's convenience we repeat the proof here.

4.2. Lemma. If $T \in \mathscr{R}_{m}(X ; G)$ is almost monotonic in $A \subset X \backslash \operatorname{spt}(\partial T)$ with gauge $\xi:(0, \delta] \rightarrow \mathbb{R}_{+}$, then $T$ is nearly monotonic in $A$ with gauge $\xi_{*}:=\mathbf{c}_{4.2} \xi$, for some constant $\mathbf{c}_{4.2}(m, \delta, \xi(\delta), \mathbf{M}(T))>0$.

Proof. For $x \in A$ and $0<r<\min \{\delta$, $\operatorname{dist}(x, \operatorname{spt}(\partial T))\}$ we abbreviate again $f_{x}(r):=$ $\boldsymbol{\alpha}(m)^{-1} r^{-m}\|T\|(\mathbf{B}(x, r))$. Because $T$ is almost monotonic,

$$
f_{x}(r) \leq \mathbf{c}_{1}:=\exp (\xi(\delta)) \frac{\mathbf{M}(T)}{\boldsymbol{\alpha}(m) \delta^{m}} .
$$

Let $\mathbf{c}_{2}:=\xi(\delta)^{-1}(\exp (\xi(\delta))-1)$. Then for $x \in A$ and $0<r \leq s \leq \delta$,

$$
\begin{aligned}
f_{x}(r) & \leq \exp (\xi(r)) f_{x}(r) \leq \exp (\xi(s)) f_{x}(s) \\
& \leq\left(1+\mathbf{c}_{2} \xi(s)\right) f_{x}(s) \leq f_{x}(s)+\mathbf{c}_{1} \mathbf{c}_{2} \xi(s) .
\end{aligned}
$$


A rather simple consequence of nearly monotonicity and lower density bounds is the compactness of the support away from the boundary and Ahlfors-regularity of the associated measure. The latter observation will be important in connection with Lemma 2.6 about tangent planes.

4.3. Lemma. Let $T \in \mathscr{R}_{m}(X ; G)$ and $U \subset X$ be an open set with $\operatorname{dist}(U$, $\operatorname{spt}(\partial T))>$ $r_{0}>0$. Assume that there is a constant $\theta>0$ such that:

(1) $\Theta^{m}(\|T\|, x) \geq \theta$ for $\|T\|$-almost all $x \in \operatorname{cl}(U)$.

(2) $\operatorname{exc}_{*}^{m}\left(\|T\|, x, r_{0}\right) \leq \frac{\theta}{2}$ for all $x \in \operatorname{cl}(U)$.

(3) $T$ is nearly monotonic in $\operatorname{cl}(U)$.

Then $\operatorname{spt}(T) \cap \operatorname{cl}(U)$ is compact and for all $0<r<r_{0}$ and $x \in \operatorname{spt}(T) \cap \operatorname{cl}(U)$ there holds

$$
\frac{\theta}{2} \leq \frac{\|T\|(\boldsymbol{B}(x, r))}{\boldsymbol{\alpha}(m) r^{m}} \leq \mathbf{c}\left(\mathbf{M}(T), r_{0}, \theta, m\right)
$$

Proof. As a closed subset of a complete space, $\operatorname{spt}(T) \cap \operatorname{cl}(U)$ is itself complete. It remains to show that this set is totally bounded. By assumption, for $\|T\|$-almost all $x \in \operatorname{cl}(U)$ and all $0<r<r_{0}$,

$$
\theta \leq \Theta^{m}(\|T\|, x) \leq \frac{\|T\|(\mathbf{B}(x, r))}{\boldsymbol{\alpha}(m) r^{m}}+\frac{\theta}{2}
$$

and hence $\frac{\theta}{2} \boldsymbol{\alpha}(m) r^{m} \leq\|T\|(\mathbf{B}(x, r))$. The nearly monotonicity of $T$ and Lemma 4.1 show that $(4.1)$ holds for every $x \in \operatorname{spt}(T) \cap \operatorname{cl}(U)$. Let $x_{1}, \ldots, x_{k}$ be points in $\operatorname{spt}(T) \cap \operatorname{cl}(U)$ with $d\left(x_{i}, x_{j}\right)>2 r$ for $i \neq j$. Then

$$
k \frac{\theta}{2} \boldsymbol{\alpha}(m) r^{m} \leq \sum_{i=1}^{k}\|T\|\left(\mathbf{B}\left(x_{i}, r\right)\right) \leq \mathbf{M}(T) .
$$

Thus there is an upper bound on the number of points that are $2 r$-separated. Taking a maximal collection of such points it follows that $\operatorname{spt}(T) \cap \operatorname{cl}(U)$ can be covered by $\left\lfloor\mathbf{M}(T)\left(\frac{\theta}{2} \boldsymbol{\alpha}(m) r^{m}\right)^{-1}\right\rfloor$ closed balls of radius $2 r$. This is true for any $r<r_{0}$ and $\operatorname{spt}(T) \cap \operatorname{cl}(U)$ is therefore totally bounded and hence compact.

The first inequality of the second conclusion follows from (4.1). Finally, for all $x \in \operatorname{spt}(T) \cap \operatorname{cl}(U)$ and $0<r<r_{0}$,

$$
\frac{\|T\|(\mathbf{B}(x, r))}{\boldsymbol{\alpha}(m) r^{m}} \leq \frac{\|T\|\left(\mathbf{B}\left(x, r_{0}\right)\right)}{\boldsymbol{\alpha}(m) r_{0}^{m}}+\mathbf{e x c}_{*}^{m}\left(\|T\|, x, r_{0}\right) \leq \frac{\mathbf{M}(T)}{\boldsymbol{\alpha}(m) r_{0}^{m}}+\frac{\theta}{2}
$$

4.2. Moments computations. Let $\phi$ be a finite Borel measure on $X$. As defined in the introduction of the seminal paper [18] by Preiss and later used in [11], we define some integrals for $r>0$, compare with $[11, \S 4.1]$. First,

$$
V(\phi, x, r):=\int_{\mathbf{B}(0, r)}\left(r^{2}-|x-y|^{2}\right)^{2} d \phi(y) .
$$


This can be written as a sum $V(\phi, x, r)=\sum_{k=0}^{4} P_{k}(\phi, x, r)$, where

$$
\begin{aligned}
P_{0}(\phi, x, r) & :=\int_{\mathbf{B}(0, r)}\left(r^{2}-|y|^{2}\right)^{2} d \phi(y), \\
P_{1}(\phi, x, r) & :=4\left\langle x, \int_{\mathbf{B}(0, r)} y\left(r^{2}-|y|^{2}\right) d \phi(y)\right\rangle, \\
P_{2}(\phi, x, r) & :=4 \int_{\mathbf{B}(0, r)}\langle x, y\rangle^{2} d \phi(y)-2|x|^{2} \int_{\mathbf{B}(0, r)} r^{2}-|y|^{2} d \phi(y), \\
P_{3}(\phi, x, r) & :=-4|x|^{2} \int_{\mathbf{B}(0, r)}\langle x, y\rangle d \phi(y), \\
P_{4}(\phi, x, r) & :=|x|^{4} \phi(\mathbf{B}(0, r)) .
\end{aligned}
$$

We further define

$$
\begin{aligned}
b(\phi, r) & :=\int_{\mathbf{B}(0, r)} y\left(r^{2}-|y|^{2}\right) d \phi(y), \\
Q(\phi, r)(x) & :=\int_{\mathbf{B}(0, r)}\langle x, y\rangle^{2} d \phi(y) .
\end{aligned}
$$

We have already encountered the quadratic form $Q$ in Subsection 2.5 although with a particular renormalization. This same renormalization of the quantities above is what we define next and use boldface letters for those. Let $\boldsymbol{\nu}(m):=\frac{\boldsymbol{\alpha}(m)}{m+2}$ and define:

$$
\begin{aligned}
\mathbf{V}(\phi, x, r) & :=\boldsymbol{\nu}(m)^{-1} r^{-m-2} V(\phi, x, r), \\
\mathbf{P}_{k}(\phi, x, r) & :=\boldsymbol{\nu}(m)^{-1} r^{-m-2} P_{k}(\phi, x, r), k=0, \ldots, 4, \\
\mathbf{b}(\phi, r) & :=\boldsymbol{\nu}(m)^{-1} r^{-m-2} b(\phi, r), \\
\mathbf{Q}(\phi, r) & :=\boldsymbol{\nu}(m)^{-1} r^{-m-2} Q(\phi, r) .
\end{aligned}
$$

Further let

$$
\boldsymbol{\omega}(m, q):=\int_{\mathbf{B}^{m}(0,1)}\left(1-|y|^{2}\right)^{q} d \mathscr{L}^{m}(y), \quad q=0,1,2, \ldots .
$$

The following simple identities will be important,

$$
\frac{\boldsymbol{\alpha}(m)}{m+2}=\boldsymbol{\nu}(m)=\frac{\boldsymbol{\omega}(m, 0)-\boldsymbol{\omega}(m, 1)}{m}=\frac{\boldsymbol{\alpha}(m)-\boldsymbol{\omega}(m, 1)}{m},
$$

and hence

$$
\boldsymbol{\omega}(m, 1)=\frac{2 \boldsymbol{\alpha}(m)}{m+2}=2 \boldsymbol{\nu}(m) .
$$

The following results in this section are from [11], the proofs below are almost the same with the minor difference that $\xi$ is a continuous increasing function for which we don't require that $\lim _{r \rightarrow 0} \xi(r)=0$. This will be important when applied in Lemma 5.8, where only small bounds on the spherical excess are assumed. For the reader's convenience we repeat the proofs here.

4.4. Lemma. [11, Lemma 4.1.1] Let $x \in X$ and $r, \epsilon>0$ be such that

$$
\left|\frac{\phi(B(x, \rho))}{\boldsymbol{\alpha}(m) \rho^{m}}-1\right| \leq \epsilon
$$


for every $0<\rho<r$. Then for every $q=0,1,2, \ldots$ one has

$$
\left|\int_{\boldsymbol{B}(x, r)}\left(r^{2}-|x-y|^{2}\right)^{q} d \phi(y)-\boldsymbol{\omega}(m, q) r^{2 q+m}\right| \leq \epsilon \boldsymbol{\omega}(m, q) r^{2 q+m} .
$$

Proof. It suffices to observe that with Cavalieri's principle,

$$
\begin{aligned}
\int_{\mathbf{B}(x, r)}\left(r^{2}-|x-y|^{2}\right)^{q} d \phi(y) & =\int_{0}^{r^{2 q}} \phi\left(\mathbf{B}\left(x,\left(r^{2}-t^{\frac{1}{q}}\right)^{\frac{1}{2}}\right)\right) d \mathscr{L}^{1}(t) \\
& \leq(1+\epsilon) \int_{0}^{r^{2 q}} \mathscr{L}^{m}\left(\mathbf{B}\left(0,\left(r^{2}-t^{\frac{1}{q}}\right)^{\frac{1}{2}}\right)\right) d \mathscr{L}^{1}(t) \\
& =(1+\epsilon) \int_{\mathbf{B}^{m}(0, r)}\left(r^{2}-|y|^{2}\right)^{q} d \mathscr{L}^{m}(y) \\
& =(1+\epsilon) \boldsymbol{\omega}(m, q) r^{2 q+m}
\end{aligned}
$$

The other inequality is proved exactly the same way.

Next is an a priori bound on the trace of $\mathbf{Q}(\phi, r)$.

4.5. Lemma. [11, Lemma 4.1.2] Let $r, \epsilon>0$ be such that

$$
\left|\frac{\phi(B(0, \rho))}{\boldsymbol{\alpha}(m) \rho^{m}}-1\right| \leq \epsilon
$$

for every $0<\rho<r$. Then

$$
|\operatorname{tr} \mathbf{Q}(\phi, r)-m| \leq \epsilon(m+4) .
$$

Proof. Let $e_{1}, e_{2}, \ldots$ be an orthonormal basis of $X$. Then

$$
\operatorname{tr} Q(\phi, r)=\sum_{i \geq 1} Q(\phi, r)\left(e_{i}\right)=\int_{\mathbf{B}(0, r)}|y|^{2} d \phi(y) .
$$

Therefore we have with (4.2) and Lemma 4.4,

$$
\begin{aligned}
\left|m \boldsymbol{\nu}(m) r^{m+2}-\operatorname{tr} Q(\phi, r)\right|= & \left.\left|m \boldsymbol{\nu}(m) r^{m+2}-\int_{\mathbf{B}(0, r)}\right| y\right|^{2} d \phi(y) \mid \\
\leq & \left|\boldsymbol{\omega}(m, 1) r^{m+2}-\int_{\mathbf{B}(0, r)}\left(r^{2}-|y|^{2}\right) d \phi(y)\right| \\
& +\left|\boldsymbol{\omega}(m, 0) r^{m+2}-r^{2} \phi(\mathbf{B}(0, r))\right| \\
\leq & \epsilon \boldsymbol{\omega}(m, 1) r^{m+2}+\epsilon \boldsymbol{\omega}(m, 0) r^{m+2} .
\end{aligned}
$$

Dividing both sides by $\boldsymbol{\nu}(m) r^{m+2}$ gives the result.

In Proposition 4.12 the quadratic form $\mathbf{Q}(\phi, r)$ is controlled in terms of the excess. In order to do so we need some bound on the length of $\mathbf{b}(\phi, r)$ in terms of the spherical excess. Define

$$
\hat{V}(\phi, x, r):=\int_{\mathbf{B}(x, r)}\left(r^{2}-|x-y|^{2}\right)^{2} d \phi(y) .
$$


4.6. Lemma. [11, Lemma 4.2.1] There is a constant $\mathbf{c}_{4.6}(m)>0$ with the following property. Whenever $2|x|<r$, then

$$
\begin{aligned}
|V(\phi, x, r)-\hat{V}(\phi, x, r)| \leq \mathbf{c}_{4.6}(m) \boldsymbol{\nu}(m) r^{m}( & r|x|^{3}\left(r^{-m} \phi(\boldsymbol{B}(0, r))\right) \\
& \left.+r^{2}|x|^{2} \mathbf{e x c}^{m *}(\phi, 0,2 r)\right) .
\end{aligned}
$$

Proof. The following statements are easy to check:

(1) $(\mathbf{B}(x, r) \backslash \mathbf{B}(0, r)) \cup(\mathbf{B}(0, r) \backslash \mathbf{B}(x, r)) \subset \mathbf{B}(x, r+|x|) \backslash \mathbf{B}(x, r-|x|)$;

(2) If $y \in(\mathbf{B}(x, r) \backslash \mathbf{B}(0, r)) \cup(\mathbf{B}(0, r) \backslash \mathbf{B}(x, r))$, then $\left|r^{2}-\right| x-\left.y\right|^{2}|\leq 3 r| x \mid$;

(3) $\mathbf{B}(0, r-2|x|) \subset \mathbf{B}(x, r-|x|) \subset \mathbf{B}(x, r+|x|) \subset \mathbf{B}(0, r+2|x|)$.

The statements (1) and (3) are obvious. To see (2), note that because of (1),

$$
0 \leq r-|x| \leq|x-y| \leq r+|x|,
$$

whence

$$
r^{2}-2 r|x|+|x|^{2} \leq|x-y|^{2} \leq r^{2}+2 r|x|+|x|^{2} .
$$

Statement (2) now follows by noting that $|x|^{2} \leq r|x|$. Using these properties we see that

$$
\begin{aligned}
|V(\phi, x, r)-\hat{V}(\phi, x, r)| & \leq 9 r^{2}|x|^{2} \phi[(\mathbf{B}(x, r) \backslash \mathbf{B}(0, r)) \cup(\mathbf{B}(0, r) \backslash \mathbf{B}(x, r))] \\
& \leq 9 r^{2}|x|^{2}(\phi(\mathbf{B}(x, r+|x|))-\phi(\mathbf{B}(x, r-|x|))) \\
& \leq 9 r^{2}|x|^{2}(\phi(\mathbf{B}(0, r+2|x|))-\phi(\mathbf{B}(0, r-2|x|))) .
\end{aligned}
$$

Since $r+2|x|<2 r$, we have that

$$
\frac{\phi(\mathbf{B}(0, r+2|x|))}{\boldsymbol{\alpha}(m)(r+2|x|)^{m}} \leq \frac{\phi(\mathbf{B}(0, r))}{\boldsymbol{\alpha}(m) r^{m}}+\mathbf{e x c}^{m *}(\phi, 0,2 r),
$$

so that

$$
\phi(\mathbf{B}(0, r+2|x|)) \leq\left(1+\frac{2|x|}{r}\right)^{m} \phi(\mathbf{B}(0, r))+\boldsymbol{\alpha}(m)(r+2|x|)^{m} \mathbf{e x c}^{m *}(\phi, 0,2 r) .
$$

Similarly,

so that

$$
\frac{\phi(\mathbf{B}(0, r-2|x|))}{\boldsymbol{\alpha}(m)(r-2|x|)^{m}} \geq \frac{\phi(\mathbf{B}(0, r))}{\boldsymbol{\alpha}(m) r^{m}}-\mathbf{e x c}^{m *}(\phi, 0, r),
$$

$$
\phi(\mathbf{B}(0, r-2|x|)) \geq\left(1-\frac{2|x|}{r}\right)^{m} \phi(\mathbf{B}(0, r))-\boldsymbol{\alpha}(m)(r-2|x|)^{m} \mathbf{e x c}^{m *}(\phi, 0, r) .
$$

From this we deduce that

$$
\begin{aligned}
\phi(\mathbf{B}(0, r+2|x|))- & \phi(\mathbf{B}(0, r-2|x|)) \\
\leq & \phi(\mathbf{B}(0, r))\left(\left(1+\frac{2|x|}{r}\right)^{m}-\left(1-\frac{2|x|}{r}\right)^{m}\right) \\
& +\left(1+2^{m}\right) \boldsymbol{\alpha}(m) r^{m} \mathbf{e x c}^{m *}(\phi, 0,2 r) \\
\leq & \phi(\mathbf{B}(0, r)) m 2^{m} \frac{2|x|}{r}+\left(1+2^{m}\right) \boldsymbol{\alpha}(m) r^{m} \operatorname{exc}^{m *}(\phi, 0,2 r) .
\end{aligned}
$$

Plugging this into (4.4) gives the result.

4.7. Definition. Given $x_{1}, x_{2} \in X$ and $r>0$ we define the deviation as

$$
\operatorname{dev}^{m}\left(\phi, x_{1}, x_{2}, r\right):=\frac{\phi\left(\mathbf{B}\left(x_{1}, r\right)\right)-\phi\left(\mathbf{B}\left(x_{2}, r\right)\right)}{r^{m}} .
$$


4.8. Lemma. [11, Lemma 4.2.3] There is a constant $\mathbf{c}_{4.8}(m)>0$ such that

$$
\begin{array}{r}
\hat{V}(\phi, x, r)-\hat{V}(\phi, 0, r) \leq \mathbf{c}_{4.8}(m) \boldsymbol{\nu}(m) r^{m+4}\left(\mathbf{d e v}^{m}(\phi, x, 0, r)\right. \\
\left.+\mathbf{e x c}_{*}^{m}(\phi, x, r)+\mathbf{e x c}^{m *}(\phi, 0, r)\right), \\
|\hat{V}(\phi, x, r)-\hat{V}(\phi, 0, r)| \leq \mathbf{c}_{4.8}(m) \boldsymbol{\nu}(m) r^{m+4}\left(\left|\mathbf{d e v}^{m}(\phi, x, 0, r)\right|\right. \\
\left.+\mathbf{e x c}^{m}(\phi, x, r)+\mathbf{e x c}^{m}(\phi, 0, r)\right) .
\end{array}
$$

Proof. As in the proof of Lemma 4.4,

$$
\begin{aligned}
\hat{V}(\phi, x, r) & =\int_{\mathbf{B}(x, r)}\left(r^{2}-|x-y|^{2}\right)^{2} d \phi(y) \\
& =\int_{0}^{r^{2}} \phi\left(\mathbf{B}\left(x,\left(r^{2}-t^{\frac{1}{2}}\right)^{\frac{1}{2}}\right)\right) d \mathscr{L}^{1}(t) \\
& =\int_{0}^{r} \phi(\mathbf{B}(x, \rho)) 4 \rho\left(r^{2}-\rho^{2}\right) d \mathscr{L}^{1}(\rho) .
\end{aligned}
$$

Similarly for $\hat{V}(\phi, 0, r)$, so that

$$
\hat{V}(\phi, x, r)-\hat{V}(\phi, 0, r)=\int_{0}^{r}(\phi(\mathbf{B}(x, \rho))-\phi(\mathbf{B}(0, \rho))) 4 \rho\left(r^{2}-\rho^{2}\right) d \mathscr{L}^{1}(\rho) .
$$

For $0<\rho<r$ we have

$$
\frac{\phi(\mathbf{B}(x, \rho))}{\boldsymbol{\alpha}(m) \rho^{m}} \leq \frac{\phi(\mathbf{B}(x, r))}{\boldsymbol{\alpha}(m) r^{m}}+\mathbf{e x c}_{*}^{m}(\phi, x, r)
$$

so that

$$
\phi(\mathbf{B}(x, \rho)) \leq \rho^{m} \frac{\phi(\mathbf{B}(x, r))}{r^{m}}+\boldsymbol{\alpha}(m) \rho^{m} \mathbf{e x c}_{*}^{m}(\phi, x, r)
$$

Similarly,

$$
-\phi(\mathbf{B}(0, \rho)) \leq-\rho^{m} \frac{\phi(\mathbf{B}(0, r))}{r^{m}}+\boldsymbol{\alpha}(m) \rho^{m} \mathbf{e x c}^{m *}(\phi, 0, r) .
$$

One also checks that

$$
\int_{0}^{r} 4 \rho^{m+1}\left(r^{2}-\rho^{2}\right) d \mathscr{L}^{1}(\rho)=8(m+2)^{-1}(m+4)^{-1} r^{m+4} .
$$

Plugging (4.6) and (4.7) into (4.5) and using (4.8) yields the first estimate. To obtain the second it suffices to apply the first one with $x$ and 0 swapped.

Next we obtain a trivial bound on $|\mathbf{b}(\phi, r)|$ due to the normalization.

4.9. Lemma. [11, Lemma 4.3.1] There is a constant $\mathbf{c}_{4.9}(m)>0$ with the following property. If $\Theta^{m}(\phi, 0)=1$, then

$$
|\mathbf{b}(\phi, r)| \leq 2 r\left(1+\mathbf{e x c}^{m}(\phi, 0, r)\right) .
$$

Proof. It suffices to apply Lemma 4.4:

$$
\begin{aligned}
|b(\phi, r)| & \leq \int_{\mathbf{B}(0, r)}|y|\left(r^{2}-|y|^{2}\right) d \phi(y) \\
& \leq r\left(1+\operatorname{exc}^{m}(\phi, 0, r)\right) \boldsymbol{\omega}(m, 1) r^{m+2},
\end{aligned}
$$

and divide by $\boldsymbol{\nu}(m) r^{m+2}$.

We will also need to control the deviation in the following way. 
4.10. Lemma. [11, Lemma 4.3.2] Assume that $\Theta^{m}(\phi, 0)=1,0<r \leq R$ and $|x|=\epsilon R$ for some $0<\epsilon \leq 1$. Then

$$
\begin{aligned}
\boldsymbol{\alpha}(m)^{-1} \mathbf{d e v}^{m}(\phi, x, 0, r) \leq & m 2^{m-1} \epsilon\left(1+\mathbf{e x c}^{m}(\phi, 0,2 R)\right) \\
& +\mathbf{e x c}^{m *}(\phi, 0,2 R)+\mathbf{e x c}_{*}^{m}(\phi, x, R) .
\end{aligned}
$$

Proof. It suffices to compute:

$$
\begin{aligned}
& \boldsymbol{\alpha}(m)^{-1} \mathbf{d e v}^{m}(\phi, x, 0, r)=\frac{\phi(\mathbf{B}(x, r))}{\boldsymbol{\alpha}(m) r^{m}}-\frac{\phi(\mathbf{B}(0, r))}{\boldsymbol{\alpha}(m) r^{m}} \\
& \leq \frac{\phi(\mathbf{B}(x, R))}{\boldsymbol{\alpha}(m) R^{m}}+\mathbf{e x c}_{*}^{m}(\phi, x, R)-\frac{\phi(\mathbf{B}(0, r))}{\boldsymbol{\alpha}(m) r^{m}} \\
& \leq \frac{(R+|x|)^{m}}{R^{m}} \frac{\phi(\mathbf{B}(0, R+|x|))}{\boldsymbol{\alpha}(m)(R+|x|)^{m}}-\frac{\phi(\mathbf{B}(0, r))}{\boldsymbol{\alpha}(m) r^{m}} \\
& +\mathbf{e x c}_{*}^{m}(\phi, x, R) \\
& \leq\left((1+\epsilon)^{m}-1\right) \frac{\phi(\mathbf{B}(0, R+|x|))}{\boldsymbol{\alpha}(m)(R+|x|)^{m}} \\
& +\frac{\phi(\mathbf{B}(0, R+|x|))}{\boldsymbol{\alpha}(m)(R+|x|)^{m}}-\frac{\phi(\mathbf{B}(0, r))}{\boldsymbol{\alpha}(m) r^{m}}+\mathbf{e x c}_{*}^{m}(\phi, x, R) \\
& \leq m 2^{m-1} \epsilon\left(1+\operatorname{exc}^{m}(\phi, 0, R+|x|)\right) \\
& +\mathbf{e x c}^{m *}(\phi, 0, R+|x|)+\mathbf{e x c}_{*}^{m}(\phi, x, R)
\end{aligned}
$$

The following is an improvement on Lemma 4.9. Note that compared with [11, Proposition 4.3.3] we don't assume that $\lim _{t \rightarrow 0} \xi(t)=0$.

4.11. Lemma. [11, Proposition 4.3.3] There is a constant $\mathbf{c}_{4.11}(m)>0$ with the following property. Let $0<2 \sqrt{r} \leq r_{0} \leq 1$ and assume there is a continuous increasing function $\xi:\left(0, r_{0}\right] \rightarrow[0,1]$ with

(1) $\Theta^{m}(\phi, 0)=1$,

(2) $\operatorname{exc}_{*}^{m}(\phi, x, \rho) \leq \xi(\rho)$ for $0<\rho \leq \sqrt{r}$ and $x \in \boldsymbol{B}(0, r)$,

(3) $\mathbf{e x c}^{m}(\phi, 0, \rho) \leq \xi(\rho)$ for $0<\rho \leq 2 \sqrt{r}$.

Then

$$
|\mathbf{b}(\phi, r)| \leq \mathbf{c}_{4.11}(m) r \max \{\sqrt[4]{r}, \sqrt{\xi(2 \sqrt{r})}\} .
$$

Proof. We start by choosing $0<\gamma(m) \leq \frac{1}{8}$ and $\eta(m)$ such that

$$
\eta(m):=4 \gamma(m)-\gamma(m)^{2}\left(2 \mathbf{c}_{4.6}+8+8(m+2)\right)>0 .
$$

We define a continuous increasing function $\epsilon:\left(0, r_{0}\right] \rightarrow \mathbb{R}_{+}$by $\epsilon(\rho):=\max \{\rho, \xi(\rho)\}$. Now either $|\gamma(m) \mathbf{b}(\phi, r)| \leq r \sqrt{\epsilon(\sqrt{r})}$ or $|\gamma(m) \mathbf{b}(\phi, r)|>r \sqrt{\epsilon(\sqrt{r})}$. We will subsequently derive an estimate for $|\mathbf{b}(\phi, r)|$ in the latter case. We first observe that since $2 r \leq \sqrt{r}$,

$$
r \epsilon(2 r) \leq r \epsilon(\sqrt{r}) \leq r \sqrt{\epsilon(\sqrt{r})}<|\gamma(m) \mathbf{b}(\phi, r)| .
$$

According to Lemma 4.9, $|\mathbf{b}(\phi, r)| \leq 4 r$ and hence $|\gamma(m) \mathbf{b}(\phi, r)| \leq \frac{r}{2} \leq r$ as well. Since $\epsilon(\sqrt{r}) \geq \sqrt{r}$ we see that

$$
|\gamma(m) \mathbf{b}(\phi, r)| \leq r \leq \sqrt{r} \epsilon(2 \sqrt{r}) .
$$


According to (4.10) and (4.11), the intermediate value theorem applied to the function

$$
[r, \sqrt{r}] \rightarrow \mathbb{R}_{+}, \rho \mapsto \rho \epsilon(2 \rho)
$$

ensures that there exists some $r \leq R \leq \sqrt{r}$ with $R \epsilon(2 R)=|\gamma(m) \mathbf{b}(\phi, r)|$. For the point $x:=\gamma(m) \mathbf{b}(\phi, r)$ we have $x \in \mathbf{B}\left(0, \frac{r}{2}\right)$. Since $P_{0}(\phi, x, r)=V(\phi, 0, r)=$ $\hat{V}(\phi, 0, r)$ we deduce from Lemma 4.6, Lemma 4.8 and Lemma 4.10 together with $|x|=R \epsilon(2 R)$ that

$$
\begin{aligned}
P_{1}(\phi, x, r)+ & P_{2}(\phi, x, r)+P_{3}(\phi, x, r)+P_{4}(\phi, x, r) \\
= & V(\phi, x, r)-P_{0}(\phi, x, r) \\
\leq & |V(\phi, x, r)-\hat{V}(\phi, x, r)|+\hat{V}(\phi, x, r)-\hat{V}(\phi, 0, r) \\
\leq & \mathbf{c}_{4.6}(m) \boldsymbol{\nu}(m) r^{m}\left(2 r|x|^{3}+r^{2}|x|^{2}\right) \\
& +\mathbf{c}_{4.8}(m) \boldsymbol{\nu}(m) r^{m+4}\left(\boldsymbol{\alpha}(m) m 2^{m-1} \epsilon(2 R) 2\right. \\
& \left.\quad+(1+\boldsymbol{\alpha}(m))\left(\operatorname{exc}^{m *}(\phi, 0,2 R)+\operatorname{exc}_{*}^{m}(\phi, x, R)\right)\right) .
\end{aligned}
$$

Define

$$
\mathbf{c}(m):=3 \max \left\{\boldsymbol{\alpha}(m) m 2^{m}, \boldsymbol{\alpha}(m)+1\right\},
$$

and divide the estimate above by $\boldsymbol{\nu}(m) r^{m+2}$. Recalling the definition of $\epsilon$, hypotheses (2), (3) and $2|x| \leq r$,

$$
\begin{aligned}
\mathbf{P}_{1}(\phi, x, r)+ & \mathbf{P}_{2}(\phi, x, r)+\mathbf{P}_{3}(\phi, x, r)+\mathbf{P}_{4}(\phi, x, r) \\
\leq & |x|^{2} \mathbf{c}_{4.6}(m)\left(\frac{2|x|}{r}+1\right) \\
& \left.+\mathbf{c}_{4.8}(m) 3^{-1} \mathbf{c}(m) r^{2}\left(\epsilon(2 R)+\mathbf{e x c}^{m *}(\phi, 0,2 R)+\mathbf{e x c}_{*}^{m}(\phi, x, R)\right)\right) \\
\leq & |x|^{2} 2 \mathbf{c}_{4.6}(m)+\mathbf{c}_{4.8}(m) \mathbf{c}(m) r^{2} \epsilon(2 R) .
\end{aligned}
$$

We further observe that according to Lemma 4.4,

$$
\begin{aligned}
\mathbf{P}_{2}(\phi, x, r) & \geq-2|x|^{2} \boldsymbol{\nu}(m)^{-1} r^{-m-2} \int_{\mathbf{B}(0, r)} r^{2}-|y|^{2} d \phi(y) \\
& \geq-8|x|^{2},
\end{aligned}
$$

as well as,

$$
\begin{aligned}
\left|\mathbf{P}_{3}(\phi, x, r)\right| & \leq 4|x|^{2} \boldsymbol{\nu}(m)^{-1} r^{-m-2} \int_{\mathbf{B}(0, r)}|x|^{2}|y|^{2} d \phi(y) \\
& \leq 8(m+2)|x|^{2},
\end{aligned}
$$

and $\mathbf{P}_{4}(\phi, x, r) \geq 0$. Together with (4.12) this yields

$$
\mathbf{P}_{1}(\phi, x, r) \leq|x|^{2}\left(2 \mathbf{c}_{4.6}(m)+8+8(m+2)\right)+\mathbf{c}_{4.8}(m) \mathbf{c}(m) r^{2} \epsilon(2 R) .
$$

Finally recall that $x=\gamma(m) \mathbf{b}(\phi, r)$ so that $|x|^{2}=\gamma(m)^{2}|\mathbf{b}(\phi, r)|^{2}$ and

$$
\begin{aligned}
\mathbf{P}_{1}(\phi, x, r) & =\frac{4}{\boldsymbol{\nu}(m) r^{m+2}}\left\langle x, \int_{\mathbf{B}(0, r)} y\left(r^{2}-|y|^{2}\right) d \phi(y)\right\rangle \\
& =4\langle x, \mathbf{b}(\phi, r)\rangle=4 \gamma(m)|\mathbf{b}(\phi, r)|^{2} .
\end{aligned}
$$

Therefore, by (4.9), (4.13) becomes,

$$
\eta(m)|\mathbf{b}(\phi, r)|^{2} \leq \mathbf{c}_{4.8}(m) \mathbf{c}(m) r^{2} \epsilon(2 R),
$$


and in turn with (4.10):

$$
|\mathbf{b}(\phi, r)| \leq \sqrt{\eta(m)^{-1} \mathbf{c}_{4.8}(m) \mathbf{c}(m)} r \sqrt{\epsilon(2 \sqrt{r})} .
$$

We recall that according to the initial dichotomy either (4.14) holds true or

$$
|\mathbf{b}(\phi, r)| \leq \gamma(m)^{-1} r \sqrt{\epsilon(\sqrt{r})} .
$$

This proves the lemma.

The following proposition is the key estimate of these moment computations.

4.12. Proposition. [11, Proposition 4.4.1] There is a constant $\mathbf{c}_{4.12}(m)>0$ with the following property. Let $x \in X, 0<2 \sqrt{r} \leq r_{0} \leq 1$ and $\xi:\left(0, r_{0}\right] \rightarrow[0,1]$ be a continuous increasing function and assume that

(1) $\Theta^{m}(\phi, 0)=\Theta^{m}(\phi, x)=1$,

(2) $|x|=r \max \{\sqrt[8]{r}, \sqrt[4]{\xi(2 \sqrt{r})}\}$,

(3) $\operatorname{exc}_{*}^{m}(\phi, y, \rho) \leq \xi(\rho)$ for $0<\rho \leq \sqrt{r}$ and $y \in \boldsymbol{B}(0, r)$,

(4) $\operatorname{exc}^{m}(\phi, y, \rho) \leq \xi(\rho)$ for $0<\rho \leq 2 \sqrt{r}$ and $y \in\{0, x\}$.

Then

$$
\left.\left.|\mathbf{Q}(\phi, r)(x)-| x\right|^{2}\left|\leq \mathbf{c}_{4.12}(m)\right| x\right|^{2} \max \{\sqrt[8]{r}, \sqrt[4]{\xi(2 \sqrt{r})}\} .
$$

Proof. First note that as in the proof of Lemma 4.11 above, Lemma 4.6 together with Lemma 4.8 imply that

$$
\begin{aligned}
\mid P_{1}(\phi, x, r)+ & P_{2}(\phi, x, r)+P_{3}(\phi, x, r)+P_{4}(\phi, x, r) \mid \\
= & \left|V(\phi, x, r)-P_{0}(\phi, x, r)\right| \\
\leq & |V(\phi, x, r)-\hat{V}(\phi, x, r)|+|\hat{V}(\phi, x, r)-\hat{V}(\phi, 0, r)| \\
\leq & \mathbf{c}_{4.6}(m) \boldsymbol{\nu}(m) r^{m}\left(r|x|^{3}(1+\xi(r))+r^{2}|x|^{2} \xi(2 r)\right) \\
& +\mathbf{c}_{4.8}(m) \boldsymbol{\nu}(m) r^{m+4}\left(\left|\operatorname{dev}^{m}(\phi, x, 0, r)\right|+2 \xi(r)\right) .
\end{aligned}
$$

Next we estimate $\left|\operatorname{dev}^{m}(\phi, x, 0, r)\right|$ by

$$
\begin{aligned}
\left|\boldsymbol{\alpha}(m)^{-1} \mathbf{d e v}^{m}(\phi, x, 0, r)\right| & =\left|\frac{\phi(\mathbf{B}(0, r))}{\boldsymbol{\alpha}(m) r^{m}}-\frac{\phi(\mathbf{B}(x, r))}{\boldsymbol{\alpha}(m) r^{m}}\right| \\
& \leq\left|\frac{\phi(\mathbf{B}(0, r))}{\boldsymbol{\alpha}(m) r^{m}}-\Theta^{m}(\phi, 0)\right|+\left|\Theta^{m}(\phi, x)-\frac{\phi(\mathbf{B}(x, r))}{\boldsymbol{\alpha}(m) r^{m}}\right| \\
& \leq \mathbf{e x c}^{m}(\phi, 0, r)+\mathbf{e x c}^{m}(\phi, x, r) \\
& \leq 2 \xi(r) .
\end{aligned}
$$

To simplify the writing, we introduce the following notation:

$$
\eta(r):=\max \{\sqrt[8]{r}, \sqrt[4]{\xi(2 \sqrt{r})}\} .
$$


Dividing (4.15) by $\boldsymbol{\nu}(m) r^{m+2}$ and using (4.16) and hypothesis (2), we obtain

$$
\begin{aligned}
\mid \mathbf{P}_{1}(\phi, x, r)+ & \mathbf{P}_{2}(\phi, x, r)+\mathbf{P}_{3}(\phi, x, r)+\mathbf{P}_{4}(\phi, x, r) \mid \\
\leq & \mathbf{c}_{4.6}(m)|x|^{2}\left(\frac{|x|}{r}(1+\xi(r))+\xi(2 r)\right) \\
& +\mathbf{c}_{4.8}(m) r^{2}\left(\left|\mathbf{d e v}^{m}(\phi, x, 0, r)\right|+2 \xi(r)\right) \\
\leq & \mathbf{c}_{4.6}(m)|x|^{2}(\eta(r)(1+\xi(r))+\xi(2 r)) \\
& +\mathbf{c}_{4.8}(m) 2(1+\boldsymbol{\alpha}(m))|x|^{2} \eta(r)^{-2} \xi(r) .
\end{aligned}
$$

According to Lemma 4.11 we also have that

$$
\begin{aligned}
\left|\mathbf{P}_{1}(\phi, x, r)\right| & =4|\langle x, \mathbf{b}(\phi, r)\rangle| \\
& \leq 4 \mathbf{c}_{4.11}(m)|x| r \max \{\sqrt[4]{r}, \sqrt{\xi(2 \sqrt{r})}\} \\
& =4 \mathbf{c}_{4.11}(m)|x|^{2} \eta(r) .
\end{aligned}
$$

Furthermore,

$$
\begin{aligned}
\left|\mathbf{P}_{3}(\phi, x, r)\right| & \leq 4|x|^{2} \boldsymbol{\nu}(m)^{-1} r^{-m-2} \int_{\mathbf{B}(0, r)}|x||y| d \phi(y) \\
& \leq 4|x|^{2} \boldsymbol{\nu}(m)^{-1} r^{-m-2} r \eta(r) r \phi(\mathbf{B}(0, r)) \\
& \leq 4(m+2)|x|^{2} \eta(r)(1+\xi(r)),
\end{aligned}
$$

as well as

$$
\begin{aligned}
\left|\mathbf{P}_{4}(\phi, x, r)\right| & =|x|^{4} \boldsymbol{\nu}(m)^{-1} r^{-m-2} \phi(\mathbf{B}(0, r)) \\
& \leq|x|^{2} \eta(r)^{2} r^{2} \boldsymbol{\nu}(m)^{-1} r^{-m-2} \phi(\mathbf{B}(0, r)) \\
& \leq(m+2)|x|^{2} \eta(r)^{2}(1+\xi(r)) .
\end{aligned}
$$

Plugging (4.18),(4.19) and (4.20) into (4.17), and observing that $\xi(2 r) \leq \eta(r)$ as well as $\eta(r)^{-2} \xi(r) \leq \sqrt{\xi(r)}$, we find that

$$
\begin{aligned}
\left|\mathbf{P}_{2}(\phi, x, r)\right| \leq & \mathbf{c}_{4.6}(m)|x|^{2}(\eta(r)(1+\xi(r))+\xi(2 r)) \\
& +2(1+\boldsymbol{\alpha}(m)) \mathbf{c}_{4.8}(m)|x|^{2} \eta(r)^{-1} \xi(r) \\
& +4 \mathbf{c}_{4.11}(m)|x|^{2} \eta(r) \\
& +5(m+2)|x|^{2} \eta(r)(1+\xi(r)) \\
\leq & \mathbf{c}(m)|x|^{2} \eta(r),
\end{aligned}
$$

for some $\mathbf{c}(m)>0$ depending only on $m$. Finally, recalling the definition of $P_{2}(\phi, x, r)$ and referring to Lemma 4.4 , it is an easy matter to check that

$$
\left.\left.4|\mathbf{Q}(\phi, r)(x)-| x\right|^{2}\left|\leq 4 \mathbf{e x c}^{m}(\phi, 0, x)\right| x\right|^{2}+\left|\mathbf{P}_{2}(\phi, x, r)\right| .
$$

Plugging (4.21) into (4.22) yields the expected estimate.

The lack of local compactness of the Hilbert space $X$ prevents us from showing that $\operatorname{spt}(\phi)$ is Reifenberg flat in a neighborhood of the origin as done in [11]. But if $\phi=\|T\|$ is Ahlfors regular we have additional structure. First we know that tangent planes exist almost everywhere by Lemma 2.6 and a slicing argument as used in the proof of Lemma 4.13 below allows us to find orthogonal frames in the support of $\phi=\|T\|$ at all small scales around a point that possesses a tangent 
plane. A priori, the closeness to a tangent plane at a given scale depends on the particular point, but the moment computations above can be used to make this scale uniform in some small neighborhood.

4.3. Uniform closeness to planes. We now show how to find an orthogonal family in the support of a rectifiable chain. This is similar to $[11$, Proposition 4.6.2], although simpler, because of the additional structure of a rectifiable chain we don't need to assume that the support is Reifenberg flat. We actually will use this family in order to show that the support of some almost monotonic chain is Reifenberg flat. Given a Radon measure $\phi$ in $X, x \in X, r>0$ and $W \in \mathbf{G}(X, m)$ we define

$$
\begin{aligned}
\beta_{2}(\phi, x, r, W) & :=\left(r^{-m-2} \int_{\mathbf{B}(x, r)}\left|\pi_{W^{\perp}}(y-x)\right|^{2} d \phi(y)\right)^{\frac{1}{2}}, \\
\beta_{\infty}(\phi, x, r, W) & :=r^{-1} \sup \left\{\left|\pi_{W^{\perp}}(y-x)\right|: y \in \operatorname{spt}(\phi) \cap \mathbf{B}(x, r)\right\} .
\end{aligned}
$$

4.13. Lemma. Let $T \in \mathscr{R}_{m}(X ; G)$ and assume that $W \in \mathbf{G}(X, m)$ and $0<\rho \leq$ $(25 \sqrt{m})^{-1}$ are such that

(1) $\operatorname{spt}(T) \subset \boldsymbol{B}(0,1)$ and $\operatorname{spt}(\partial T) \subset \partial \boldsymbol{B}(0,1)$,

(2) $\pi_{W \#}\left(T\left\llcorner Z_{W}\left(0,2^{-1}\right)\right) \neq 0\right.$,

(3) $\beta_{\infty}(\|T\|, 0,1, W)<\rho$.

Let $\rho^{\prime}:=m^{\frac{1}{4}} \rho^{\frac{1}{2}}$. Then $\rho^{\prime} \leq \frac{1}{5}$ and for every $s \in\left(2 \rho^{\prime}, 1\right]$ there $i s$ an orthonormal family $e_{1}, \ldots, e_{m} \in X$ with $s_{i} \in \operatorname{spt}(T), i=1, \ldots, m$.

Proof. Note that if $v_{1}, \ldots, v_{m} \in X$ are orthonormal vectors with $\left|\pi_{W^{\perp}}\left(v_{i}\right)\right| \leq c$ and $V:=\operatorname{span}\left\{v_{1}, \ldots, v_{m}\right\} \in \mathbf{G}(X, m)$, then

$$
d_{\mathrm{H}}\left(\mathbf{B}_{V}(0,1), \mathbf{B}_{W}(0,1)\right) \leq \sup \left\{\left|\pi_{W \perp}(x)\right|: x \in \mathbf{B}_{V}(0,1)\right\} \leq \sqrt{m} c .
$$

By hypothesis $(3)$ (and since $\left.1-\rho \leq \sqrt{1-\rho^{2}}\right), \operatorname{spt}(\partial T) \cap Z_{W}(1-\rho)=\emptyset$ and the constancy theorem implies together with $(2)$ that there is some $g_{0} \neq 0$ with

$$
\pi_{W \#}\left(T\left\llcorner Z_{W}(1-\rho)\right)=g_{0} \llbracket \mathbf{B}_{W}(0,1-\rho) \rrbracket .\right.
$$

For an $m$-plane $V$ and $r>0$ let $\psi_{V, r}: \mathbf{B}(0,1) \backslash Z_{V}(r) \rightarrow V$ be the Lipschitz map given by $\psi_{V, r}(x):=|x|\left|\pi_{V}(x)\right|^{-1} \pi_{V}(x)$. This map preserves the norm and orthogonality in the following sense,

$$
\left|\psi_{V, r}(x)\right|=|x|, \quad \text { and for } y \in V \quad \psi_{V, r}(x) \perp y \Leftrightarrow x \perp y .
$$

The fist statement is obvious, for the second note that if $x=v+v^{\perp}$ with $v \in V$ and $v \in V^{\perp}$, then $\psi_{V, r}(x)=\lambda v$ for some $\lambda \neq 0$ and hence

$$
\left\langle\psi_{V, r}(x), y\right\rangle=\lambda\langle v, y\rangle=\lambda\langle x, y\rangle,
$$

for $y \in V$. Let $H_{V, r}:[0,1] \times\left(\mathbf{B}(0,1) \backslash Z_{V}(r)\right) \rightarrow V$ be the Lipschitz homotopy $H_{V, r}(t, x)=t \pi_{V}(x)+(1-t) \psi_{V, r}(x)$ between $\psi_{V, r}$ and $\pi_{V}$. By assumption $\pi_{W}$ : $\operatorname{spt}(T) \cap Z_{W}(1-\rho) \rightarrow \mathbf{B}_{W}(0,1-\rho)$ is surjective. We next show that

$$
B_{W}(0,1) \backslash \mathbf{B}_{W}(0,2 \rho) \subset \psi_{W, \rho}\left(\operatorname{spt}(T) \backslash Z_{W}(\rho)\right) .
$$

This is essentially a consequence of the constancy theorem. More precisely, consider the rectifiable chain $S:=\llbracket 0,1 \rrbracket \times\left(T\left\llcorner Z_{W}(\rho)^{c}\right) \in \mathscr{R}_{m+1}(\mathbb{R} \times X ; G)\right.$ and the set $B_{\rho}:=\mathbf{B}_{W}(0,1-\rho) \backslash \mathbf{B}_{W}(0,2 \rho)$. Clearly, $H_{W, \rho \#} S=0$ because the image lies in an 
$m$-dimensional plane. By the homotopy formula for $G$-chains (compare with the proof of Lemma 2.7),

$$
\begin{aligned}
0 & =\partial\left(H_{W, \rho \#} S\right)=H_{W, \rho \#} \partial S \\
& =H_{W, \rho \#}\left(\llbracket 1 \rrbracket \times\left(T\left\llcorner Z_{W}(\rho)^{c}\right)-\llbracket 0 \rrbracket \times\left(T\left\llcorner Z_{W}(\rho)^{c}\right)-\llbracket 0,1 \rrbracket \times \partial\left(T\left\llcorner Z_{W}(\rho)^{c}\right)\right)\right.\right.\right. \\
& =\pi_{W \#}\left(T\left\llcorner Z_{W}(\rho)^{c}\right)-\psi_{W, \rho \#}\left(T\left\llcorner Z_{W}(\rho)^{c}\right)-H_{W, \rho \#}\left(\llbracket 0,1 \rrbracket \times \partial\left(T\left\llcorner Z_{W}(\rho)^{c}\right)\right) .\right.\right.\right.
\end{aligned}
$$

Because of hypothesis (1) and (3), $H_{W, \rho \#}\left(\operatorname{spt}\left([0,1] \times \partial\left(T\left\llcorner Z_{W}(\rho)^{c}\right)\right) \subset W \backslash B_{\rho}\right.\right.$ and on $B_{\rho},\left(\pi_{W \#}\left(T\left\llcorner Z_{W}(\rho)^{c}\right)\right)\left\llcorner B_{\rho}=g_{0} \llbracket B_{\rho} \rrbracket\right.\right.$. So the same must be true for $\psi_{W, \rho \#}\left(T\left\llcorner Z_{W}(\rho)^{c}\right)\right.$, and since $g_{0} \neq 0$, the inclusion in (4.25) holds by the constancy theorem because $\psi_{W, \rho}\left(\partial\left(T\left\llcorner Z_{W}(\rho)^{c}\right)\right) \subset \partial \mathbf{B}_{W}(0,1) \cup \mathbf{B}_{W}(0,2 \rho)\right.$.

Pick some $w_{1} \in W$ with $\left|w_{1}\right|=s$, where $s \in\left(2 \rho^{\prime}, 1\right]$. Since $2 \rho \leq 2 \rho^{\prime}<s$, (4.25) implies the existence of some $e_{1} \in X$ with $s e_{1} \in \operatorname{spt}(T)$ and $\psi_{W, \rho}\left(s e_{1}\right)=$ $w_{1}$. Because $\psi_{W, \rho}$ preserves the norm by (4.24), $e_{1}$ is of unit length. Let $V_{1}$ be the orthogonal complement of $w_{1}$ in $W$. Again by (4.24), the vector $e_{1}$ is also orthogonal to $V_{1} \subset W$. Consider the new $m$-plane $W_{1}$ spanned by $e_{1}$ and $V_{1}$. There holds $\left|\pi_{W^{\perp}}\left(s e_{1}\right)\right| \leq \rho$ because $s e_{1} \in \operatorname{spt}(T)$ and hence $d_{\mathrm{H}}\left(\mathbf{B}_{W}(0,1), \mathbf{B}_{W_{1}}(0,1)\right) \leq$ $\sqrt{m} \rho s^{-1}$ by (4.23). Accordingly,

$$
\begin{aligned}
\beta_{\infty}\left(\|T\|, 0,1, W_{1}\right) & \leq \beta_{\infty}(\|T\|, 0,1, W)+d_{\mathrm{H}}\left(\mathbf{B}_{W}(0,1), \mathbf{B}_{W_{1}}(0,1)\right) \\
& <\left(1+\sqrt{m} s^{-1}\right) \rho \leq 2 \sqrt{m} \rho s^{-1} \leq \sqrt{m} \rho \rho^{\prime-1} \\
& =\rho^{\prime} \leq 5^{-1} .
\end{aligned}
$$

Because of Lemma 2.7, the new plane $W_{1}$ satisfies the same hypotheses as $W$ with $\rho^{\prime}$ in place of $\rho$.

Assume that for $k<m$ we have already constructed some orthonormal vectors $e_{1}, \ldots, e_{k}$ orthogonal to some $m-k$ dimensional subspace $V_{k} \subset W$ with $s e_{i} \in \operatorname{spt}(T)$ for all $i$. With $W_{k}$ we denote the $m$-plane spanned by $e_{1}, \ldots, e_{k}$ and $V_{k}$. Now, pick a $w_{k+1} \in V_{k}$ with $\left|w_{k+1}\right|=s$ and $s \in\left(2 \rho^{\prime}, 1\right]$. The same calculation as for $W_{1}$ gives that

$$
\max \left\{\boldsymbol{\beta}_{\infty}\left(\|T\|, 0,1, W_{k}\right), d_{\mathrm{H}}\left(\mathbf{B}_{W}(0,1), \mathbf{B}_{W_{k}}(0,1)\right)\right\}<\rho^{\prime} \leq 5^{-1},
$$

and as in (4.25) there is some $e_{k+1} \in X$ with $s e_{k+1} \in \operatorname{spt}(T)$ and $\psi_{W_{k}, \rho^{\prime}}\left(s e_{k+1}\right)=$ $w_{k+1}$. Let $V_{k+1}$ be the orthogonal complement of $w_{k+1}$ in $V_{k}$. Since $w_{k+1}$ is orthogonal to each of the vectors $e_{1}, \ldots, e_{k}$ and to $V_{k+1}$ the same holds for $e_{k+1}$ by (4.24) applied to the map $\psi_{W_{k}, \rho^{\prime}}$. The new plane $W_{k+1}$ has the same properties we obtained for $W_{k}$. Proceeding this way we get the desired orthonormal vectors $e_{1}, \ldots, e_{m}$.

Lemma 4.5 together with Proposition 4.12 allow us to control $\boldsymbol{\beta}_{2}$ with respect to some plane that is spanned by an orthogonal frame in the support of $\phi$. This corresponds to Lemma 4.7.2 and Proposition 4.7.3 in [11].

4.14. Proposition. There is a constant $\mathbf{c}_{4.14}(m)>0$ with the following property. Let $\phi$ be a finite Borel measure on $X, x_{i}, x_{i, k} \in X$ for $i=1, \ldots, m$ and $k \geq 1$ be a sequence of points. Further assume that $0<4 \sqrt{r}<r_{0} \leq 1$ and $\xi:\left(0, r_{0}\right] \rightarrow\left(0, \frac{1}{2}\right]$ is a continuous increasing function such that

(1) $\phi(\partial \boldsymbol{B}(0, r))=0$,

(2) $\lim _{k \rightarrow \infty}\left|x_{i, k}-x_{i}\right|=0$ for all $i$, 
(3) $x_{i} \perp x_{j}$ for $i \neq j$, and $\left|x_{i}\right|=r \eta(r)$ for

$$
\eta(r):=\max \{\sqrt[8]{r}, \sqrt[4]{\xi(2 \sqrt{r})}\},
$$

(4) $\Theta^{m}(\phi, 0)=\Theta^{m}\left(\phi, x_{i, k}\right)=\Theta^{m}(\phi, y)=1$ for all $i, k$ and $\phi$-almost all $y \in$ $\boldsymbol{B}(0, r)$,

(5) $\mathbf{e x c}_{*}^{m}(\phi, y, \rho) \leq \xi(\rho)$ for $0<\rho \leq 4 \sqrt{r}$ and $y \in \boldsymbol{B}(0,2 r)$,

(6) $\operatorname{exc}^{m *}(\phi, y, \rho) \leq \xi(\rho)$ for $0<\rho \leq 4 \sqrt{r}$ and $y \in\left\{0, x_{i, k}: i, k \geq 1\right\}$.

Then

$$
\boldsymbol{\beta}_{\infty}(\phi, 0, r / 2, W)<\mathbf{c}_{4.14}(m) \eta(r)^{\frac{1}{m+2}},
$$

where $W:=\operatorname{span}\left\{x_{1}, \ldots, x_{m}\right\}$.

Proof. If $k$ is big enough, then $W_{k}:=\operatorname{span}\left\{x_{1, k}, \ldots, x_{m, k}\right\}$ is an $m$-dimensional subspace of $X$ and $W_{k}$ converges to $W$. Without loss of generality we assume that $W_{k} \in \mathbf{G}(X, m)$ for all $k$ and $0<\left|x_{i, k}\right|<2 r \eta(r) \leq 2 r$ for all $i$ and $k$. Let $e_{i}, f_{i, k} \in X$ be the unit vectors such that $\left|x_{i, k}\right| f_{i, k}=x_{i, k}$ and $\left|x_{i}\right| e_{i}=x_{i}$ and let $e_{m+1, k}, e_{m+2, k}, e_{m+3, k}, \ldots$ be an orthonormal basis of $W_{k}^{\perp}$. By assumption $s \mapsto s \eta(s)$ is continuous, strictly increasing and satisfies $\lim _{s \downarrow 0} s \eta(s)=0$. Hence there is a unique $r_{i, k}>0$ for which $\left|x_{i, k}\right|=r_{i, k} \eta\left(r_{i, k}\right)$ for some $0<r_{i, k}<2 r$ and

$$
\lim _{k \rightarrow \infty} \max _{1 \leq i \leq m}\left|r_{i, k}-r\right|=\lim _{k \rightarrow \infty} \max _{1 \leq i \leq m}\left|\eta\left(r_{i, k}\right)-\eta(r)\right|=0 .
$$

Further, there is an orthonormal basis $e_{1, k}, \ldots, e_{m, k}$ of $W_{k}$ with

$$
\lim _{k \rightarrow \infty} \max _{1 \leq i \leq m}\left|r \eta(r) e_{i, k}-x_{i, k}\right|=\lim _{k \rightarrow \infty} \max _{1 \leq i \leq m}\left|e_{i, k}-f_{i, k}\right|=0 .
$$

$e_{i, k}$ can for example be constructed via the Gram-Schmidt procedure from $e_{i, k}$. From hypothesis (1) it follows that

$$
\lim _{\epsilon \downarrow 0} \phi(\mathbf{B}(0, r+\epsilon))-\phi(\mathbf{B}(0, r-\epsilon))=0 .
$$

By the assumption on $\xi$ we have for all $0<\rho \leq r$,

$$
\left|\frac{\phi(\mathbf{B}(0, \rho))}{\boldsymbol{\alpha}(m) \rho^{m}}-1\right| \leq \max \left\{\mathbf{e x c}_{*}^{m}(\phi, 0, r), \mathbf{e x c}^{m *}(\phi, 0, r)\right\} \leq \xi(r) .
$$

Lemma 4.5 then implies that for all $k$,

$$
\left|m-\sum_{i \geq 1} \frac{1}{\boldsymbol{\nu}(m) r^{m+2}} \int_{\mathbf{B}(0, r)}\left\langle e_{i, k}, y\right\rangle^{2} d \phi(y)\right|=|m-\operatorname{tr} \mathbf{Q}(\phi, r)| \leq(m+4) \xi(r) .
$$

Additionally, Proposition 4.12 implies that for all $i, k$,

$$
\begin{aligned}
\left|1-\frac{1}{\boldsymbol{\nu}(m) r_{i, k}^{m+2}} \int_{\mathbf{B}\left(0, r_{i, k}\right)}\left\langle f_{i, k}, y\right\rangle^{2} d \phi(y)\right| & =\left.\frac{1}{\left|x_{i, k}\right|^{2}}|| x_{i, k}\right|^{2}-\mathbf{Q}\left(\phi, r_{i, k}\right)\left(x_{i, k}\right) \mid \\
& \leq \mathbf{c}_{4.12}(m) \eta\left(r_{i, k}\right) .
\end{aligned}
$$

From (4.26), (4.27) and (4.28) we get

$$
\begin{aligned}
\epsilon_{k}:=\max _{1 \leq i \leq m} & \mid \frac{1}{\boldsymbol{\nu}(m) r_{i, k}^{m+2}} \int_{\mathbf{B}\left(0, r_{i, k}\right)}\left\langle f_{i, k}, y\right\rangle^{2} d \phi(y) \\
& -\frac{1}{\boldsymbol{\nu}(m) r^{m+2}} \int_{\mathbf{B}(0, r)}\left\langle e_{i, k}, y\right\rangle^{2} d \phi(y) \mid \rightarrow 0,
\end{aligned}
$$

for $k \rightarrow \infty$. 
Since $\xi(r) \leq \eta(r)$ it follows from (4.26), (4.29), (4.30) and (4.31) that for $\mathbf{c}(m):=$ $\boldsymbol{\nu}(m)\left(m \mathbf{c}_{4.12}(m)+2 m+4\right)$,

$$
\begin{aligned}
\beta_{2}\left(\phi, 0, r, W_{k}\right)^{2} & =\frac{1}{r^{m+2}} \int_{\mathbf{B}(0, r)}\left|\pi_{W_{k}}(y)\right|^{2} d \phi(y) \\
& =\sum_{i>m} \frac{1}{r^{m+2}} \int_{\mathbf{B}(0, r)}\left\langle e_{i, k}, y\right\rangle^{2} d \phi(y) \\
& \leq \boldsymbol{\nu}(m) \operatorname{tr} \mathbf{Q}(\phi, r)-\sum_{i \leq m} \frac{1}{r^{m+2}} \int_{\mathbf{B}(0, r)}\left\langle e_{i, k}, y\right\rangle^{2} d \phi(y) \\
& \leq \boldsymbol{\nu}(m)\left((m+4) \xi(r)+m \mathbf{c}_{4.12}(m) \eta\left(r_{i, k}\right)+m \epsilon_{k}\right) \\
& \leq \mathbf{c}(m) \max _{1 \leq i \leq m}\left\{\eta(r), \eta\left(r_{i, k}\right), \epsilon_{k}\right\} \rightarrow \mathbf{c}(m) \eta(r),
\end{aligned}
$$

for $k \rightarrow \infty$. Let $\delta:=\boldsymbol{\beta}_{2}\left(\phi, 0, r, W_{k}\right)$ and define

$$
\begin{aligned}
q_{m} & :=\frac{2}{m+2}, \\
c_{m} & :=1+\left(4 \boldsymbol{\alpha}(m)^{-1}\right)^{\frac{1}{m}}, \\
\delta_{m} & :=\left(2\left(c_{m}-1\right)\right)^{-\frac{1}{q_{m}}} .
\end{aligned}
$$

If $\delta \geq \delta_{m}$, then

$$
\beta_{\infty}\left(\phi, 0, r / 2, W_{k}\right) \leq 1 \leq\left(\delta_{m}^{-1} \delta\right)^{q_{m}} \leq \delta_{m}^{-q_{m}} \delta^{q_{m}} .
$$

We therefore assume that $\delta<\delta_{m}$. Consider the set

$$
B:=\left\{y \in \mathbf{B}(0, r) \cap \operatorname{spt}(\phi):\left|\pi_{W_{k}^{\perp}}(y)\right| \geq \delta^{q_{m}} r\right\} .
$$

Observe that

$$
\begin{aligned}
\delta^{2} & =\frac{1}{r^{m+2}} \int_{\mathbf{B}(0, r)}\left|\pi_{W_{k}^{\perp}}(y)\right|^{2} d \phi(y) \\
& \geq \frac{1}{r^{m+2}} \phi(B) \delta^{2 q_{m}} r^{2}
\end{aligned}
$$

and therefore

$$
\phi(B) \leq r^{m} \delta^{2\left(1-q_{m}\right)} .
$$

Now assume there exists $y \in \mathbf{B}(0, r / 2) \cap \operatorname{spt}(\phi)$ with

$$
\left|\pi_{W_{k}^{\perp}}(y)\right|>c_{m} \delta^{q_{m}} r .
$$

Put $\rho:=\left(c_{m}-1\right) \delta^{q_{m}} r$ and notice that $\mathbf{B}(y, \rho) \subset \mathbf{U}(0, r)$ since

$$
\rho=\left(c_{m}-1\right) \delta^{q_{m}} r<\left(c_{m}-1\right) \delta_{m}^{q_{m}} r=\left(c_{m}-1\right)\left(2\left(c_{m}-1\right)\right)^{-1} r \leq \frac{1}{2} r .
$$

By our assumption on the densities we can assume that $\Theta^{m}(\phi, y)=1$. The above implies that $\operatorname{spt}(\phi) \cap \mathbf{B}(y, \rho) \subset B$ and with (4.33) we obtain

$$
\phi(\mathbf{B}(y, \rho)) \leq \phi(B) \leq r^{m} \delta^{2\left(1-q_{m}\right)} .
$$

Since $\xi(\rho) \leq \xi\left(r_{0}\right) \leq \frac{1}{2}$, the bound on $\operatorname{exc}_{*}^{m}(\phi, y, \rho)$ implies that

$$
\frac{\phi(\mathbf{B}(y, \rho))}{\boldsymbol{\alpha}(m) \rho^{m}} \geq 1-\xi(\rho) \geq \frac{1}{2}
$$


Combining this with (4.34) we obtain

$$
\frac{1}{2} \boldsymbol{\alpha}(m)\left(c_{m}-1\right)^{m} \delta^{m q_{m}} r^{m}=\frac{1}{2} \boldsymbol{\alpha}(m) \rho^{m} \leq \phi(\mathbf{B}(y, \rho)) \leq r^{m} \delta^{2\left(1-q_{m}\right)} .
$$

Since $m q_{m}=\frac{2 m}{m+2}=2\left(1-\frac{2}{m+2}\right)=2\left(1-q_{m}\right)$, this gives a contradiction,

$$
1 \geq \frac{1}{2} \boldsymbol{\alpha}(m)\left(c_{m}-1\right)^{m}=\frac{1}{2} \boldsymbol{\alpha}(m) 4 \boldsymbol{\alpha}(m)^{-1}=2 .
$$

Therefore

$$
\boldsymbol{\beta}_{\infty}\left(\phi, 0, r / 2, W_{k}\right) \leq \max \left\{\delta_{m}^{-q_{m}}, c_{m}\right\} \delta^{q_{m}}=\max \left\{2\left(c_{m}-1\right), c_{m}\right\} \delta^{\frac{2}{m+2}} .
$$

Since $\delta=\beta_{2}\left(\phi, 0, r, W_{k}\right),(4.32)$ implies that

$$
\limsup _{k \rightarrow \infty} \beta_{\infty}\left(\phi, 0, r / 2, W_{k}\right) \leq \mathbf{c}^{\prime}(m) \eta(r)^{\frac{1}{m+2}},
$$

for some constant $\mathbf{c}^{\prime}(m)>0$ depending only on $m$. Because $W_{k} \cap \mathbf{B}(0,1)$ converges in Hausdorff distance to $W \cap \mathbf{B}(0,1)$, we get $\boldsymbol{\beta}_{\infty}\left(\phi, 0, r / 2, W_{k}\right) \rightarrow \boldsymbol{\beta}_{\infty}(\phi, 0, r / 2, W)$ and the proposition follows.

This is the special case of the proposition above in case $\xi$ is the constant function.

4.15. Corollary. There is a constant $\mathbf{c}_{4.15}(m)>0$ with the following property. Let $\phi$ be a finite Borel measure on $X, x_{i}, x_{i, k} \in X$ for $i=1, \ldots, m$ and $k \geq 1$ and assume that $\epsilon, r_{0}, r>0$ are such that:

(1) $0<4 \sqrt{r} \leq r_{0} \leq \epsilon \leq \frac{1}{2}$,

(2) $\phi(\partial \boldsymbol{B}(0, r))=0$,

(3) $\lim _{k \rightarrow \infty}\left|x_{i, k}-x_{i}\right|=0$ for all $i$,

(4) $x_{i} \perp x_{j}$ for $i \neq j$ and $\left|x_{i}\right|=r \epsilon^{\frac{1}{4}}$,

(5) $\Theta^{m}(\phi, 0)=\Theta^{m}\left(\phi, x_{i, k}\right)=\Theta^{m}(\phi, y)=1$ for all $i, k$ and $\phi$-almost all $y \in$ $\boldsymbol{B}\left(0, r_{0}\right)$.

(6) $\mathbf{e x c}_{*}^{m}\left(\phi, y, r_{0}\right) \leq \epsilon$ for $y \in \boldsymbol{B}\left(0,2 r_{0}\right)$,

(7) $\operatorname{exc}^{m *}\left(\phi, y, r_{0}\right) \leq \epsilon$ for $y \in\left\{0, x_{i, k}: i, k \geq 1\right\}$.

Then

$$
\boldsymbol{\beta}_{\infty}(\phi, 0, r / 2, W)<\mathbf{c}_{4.15}(m) \epsilon^{\frac{1}{4(m+2)}},
$$

where $W:=\operatorname{span}\left\{x_{1}, \ldots, x_{m}\right\}$.

\section{Regularity of Almost minimizer}

First we define the main objects of this paper, namely almost mass minimizing rectifiable chains with respect to some gauge $\xi$. This is an adaptation of the original definition of Almgren in [2] to chains. Almgren's original definition is harder to work with since competing surfaces have to be obtained by Lipschitz deformations of the original one and cut and paste constructions are not allowed. Due to slicing, such cut and paste constructions are easily available for rectifiable $G$-chains and this simplifies the arguments greatly. For this reason we follow Bombieri and his definition of almost mass minimizing integral currents [8].

5.1. Definition. A rectifiable chain $T \in \mathscr{R}_{m}(X ; G)$ is $(\mathbf{M}, \xi, \delta)$-minimal in a set $A \subset X \backslash \operatorname{spt}(\partial T)$ if $\xi:(0, \delta] \rightarrow \mathbb{R}_{+}$is a gauge and the following holds: For every $x \in A, 0<r<\min \{\delta, \operatorname{dist}\{x, \operatorname{spt}(\partial T)\}\}$ and every $S \in \mathscr{R}_{m}(X ; G)$ with

(1) $\operatorname{spt}(S) \subset \mathbf{B}(x, r)$,

(2) $\partial S=0$, 
there holds

$$
\mathbf{M}(T\llcorner\mathbf{B}(x, r)) \leq(1+\xi(r)) \mathbf{M}(T\llcorner\mathbf{B}(x, r)+S) .
$$

We will further assume that $\xi$ is continuous and satisfies the Dini condition,

$$
\int_{0}^{\delta} \frac{\xi(t)}{t} d t<\infty
$$

The next result is a simple adjustment of [11, Proposition 3.4.5] to the setting of rectifiable $G$-chains. Note that because of the definition of almost minimality we use here, radius of balls instead of diameter of sets, there is no factor 2 appearing in the lemma below.

5.2. Lemma. Let $T \in \mathscr{R}_{m}(X ; G)$ be $(\mathbf{M}, \xi, \delta)$-minimal in $A \subset X \backslash \boldsymbol{B}(\operatorname{spt}(\partial T), \delta)$ and define

$$
\Xi(r):=m \int_{0}^{r} \frac{\xi(t)}{t} d t
$$

for every $0<r \leq \delta$. Then $T$ is almost monotonic in $A$ with gauge $\Xi$.

Proof. For $x \in A$ and $0<r<\delta$ define $f_{x}(r):=\|T\|(\mathbf{B}(x, r))$. Let $d_{x}: X \rightarrow \mathbb{R}$ be the distance function to $x$ and assume that $r$ is such that $\left\langle T, d_{x}, r\right\rangle \in \mathscr{R}_{m-1}(X ; G)$ as well as $\partial\left(T\llcorner\mathbf{B}(x, r))=\left\langle T, d_{x}, r\right\rangle \in \mathscr{R}_{m-1}(X ; G)\right.$. This holds for almost all $0<r<\delta$ because of [13, Theorem 5.2.4] and by (2.7),

$$
\int_{0}^{r} \mathbf{M}\left(\left\langle T, d_{x}, s\right\rangle\right) d s \leq \mathbf{M}\left(T\llcorner\mathbf{B}(x, r))=f_{x}(r) .\right.
$$

Hence for almost all $r$,

$$
\mathbf{M}\left(\left\langle T, d_{x}, r\right\rangle\right) \leq f_{x}^{\prime}(r) .
$$

Let $\llbracket x \rrbracket \mathbb{x}\left\langle T, d_{x}, r\right\rangle \in \mathscr{R}_{m}(X ; G)$ be the cone over $\left\langle T, d_{x}, r\right\rangle$ with center $x$. There holds $\partial\left(\llbracket x \rrbracket \nVdash\left\langle T, d_{x}, r\right\rangle\right)=\left\langle T, d_{x}, r\right\rangle$ and by $(2.8)$,

$$
\mathbf{M}\left(\llbracket x \rrbracket \circledast\left\langle T, d_{x}, r\right\rangle\right)=\frac{r}{m} \mathbf{M}\left(\left\langle T, d_{x}, r\right\rangle\right) .
$$

The almost minimality of $T$ implies in combination with (5.1) and (5.2) that for almost all $r$,

$$
\begin{aligned}
f_{x}(r) & =\mathbf{M}\left(T\llcorner\mathbf{B}(x, r)) \leq(1+\xi(r)) \mathbf{M}\left(\llbracket x \rrbracket \mathbb{x}\left\langle T, d_{x}, r\right\rangle\right)\right. \\
& =(1+\xi(r)) \frac{r}{m} \mathbf{M}\left(\left\langle T, d_{x}, r\right\rangle\right) \leq(1+\xi(r)) \frac{r}{m} f_{x}^{\prime}(r) .
\end{aligned}
$$

Hence, for almost all $r$ with $f_{x}(r)>0$,

$$
\left(\log \circ f_{x}\right)^{\prime}(r)=\frac{f_{x}^{\prime}(r)}{f_{x}(r)} \geq \frac{m}{r} \frac{1}{1+\xi(r)} \geq \frac{m}{r}(1-\xi(r)) .
$$

Integrating shows that for all $0<r_{1}<r_{2}<\delta$ with $f_{x}\left(r_{1}\right)>0$,

$$
\log \left(\frac{f_{x}\left(r_{2}\right)}{f_{x}\left(r_{1}\right)}\right) \geq \int_{r_{1}}^{r_{2}} \frac{m}{r}(1-\xi(r)) d r=\log \left(\frac{r_{2}^{m}}{r_{1}^{m}}\right)-\Xi\left(r_{2}\right)+\Xi\left(r_{1}\right),
$$

respectively that

$$
\exp \left(\Xi\left(r_{1}\right)\right) r_{1}^{-m} f_{x}\left(r_{1}\right) \leq \exp \left(\Xi\left(r_{2}\right)\right) r_{2}^{-m} f_{x}\left(r_{2}\right) .
$$

If $f_{x}\left(r_{1}\right)=0$, the statement is trivial. Hence $T$ is almost monotonic in $A$ with gauge function $\Xi$. 
We will encounter a similar differential equation in connection with the epiperimetric inequality in Lemma 5.5.

5.1. Polyhedral approximation and a differential inequality. Because of the formulation of Reifenberg's epiperimetric inequality with polyhedral chains we first need some results that justify this assumption. The required results about polyhedral approximation are contained in [10]. With $\theta>0$ and the group norm $\|\cdot\|$ we associate a new group norm $\|\cdot\|_{\theta}$ defined on $G$ by $\left\|0_{G}\right\|_{\theta}=0$ and $\|g\|_{\theta}:=\max \{\|g\|, \theta\}$ whenever $g \neq 0_{G}$.

5.3. Lemma. Let $T \in \mathscr{R}_{m}(X ; G)$ and $r_{0} \in(0,1]$ such that $\operatorname{spt}(\partial T) \cap \boldsymbol{B}\left(0, r_{0}\right)=\emptyset$. Further let $\theta>0$ and assume that,

(A) $\Theta^{m}(\|T\|, x) \geq \theta$ for $\|T\|$-a.e. $x \in \boldsymbol{B}\left(0, r_{0}\right)$,

(B) $\operatorname{spt}(T) \cap \boldsymbol{B}\left(0, r_{0}\right)$ is compact.

Let $\mathbf{M}_{\theta}$ be the mass on rectifiable $G$-chains induced by the norm $\|\cdot\|_{\theta}$ (defined right before the Lemma). For all $s>0$ and almost every $r \in\left[0, r_{0}\right], T_{r}:=$ $\partial\left(T\llcorner\boldsymbol{B}(0, r)) \in \mathscr{R}_{m-1}(X ; G)\right.$ and there is a polyhedral chain $P_{r, s} \in \mathscr{P}_{m-1}(X ; G)$ and a rectifiable chain $R_{r, s} \in \mathscr{R}_{m}(X ; G)$ such that

(1) $\partial\left(R_{r, s}+\llbracket 0 \rrbracket \mathbb{*} P_{r, s}\right)=T_{r}$,

(2) $\mathbf{M}_{\theta}\left(R_{r, s}\right)<s$ and $\operatorname{spt}\left(R_{r, s}\right) \subset \boldsymbol{U}\left(\operatorname{spt}\left(T_{r}\right), s\right)$,

(3) $\max \left\{\mathbf{M}_{\theta}\left(\bar{P}_{r, s}\right)\llcorner\boldsymbol{B}(0, r)), \mathbf{M}_{\theta}\left(\llbracket 0 \rrbracket \mathbb{*} P_{r, s}\right)\right\} \leq \mathbf{M}\left(\llbracket 0 \rrbracket \mathbb{*} T_{r}\right)+s$, where $\bar{P}_{r, s}$ is the infinite cone generated by $\llbracket 0 \rrbracket * P_{r, s}$.

Proof. Let $f: X \rightarrow \mathbb{R}$ be the 1-Lipschitz function given by $f(x):=|x|$. As noted before, the slice $\langle T, f, r\rangle$ exists for almost every $r \in \mathbb{R}$ and is an element of $\mathscr{R}_{m-1}(X ; G)$. By [13, Theorem 5.2.4] we have that $\langle T, f, r\rangle=\partial(T\llcorner\{f<r\})=$ $\partial\left(T\llcorner\mathbf{B}(0, r))=T_{r}\right.$ for almost every $r \leq r_{0}$. By the compactness assumption on $T$, the chain $T_{r}$ has also compact support. It follows from [10, Theorem 4.2 (E)] that there are $P_{r, s} \in \mathscr{P}_{m-1}(X ; G)$ and $R_{r, s} \in \mathscr{R}_{m}(X ; G)$ such that $P_{r, s}-T_{r}=\partial R_{r, s}$, $\mathbf{M}_{\theta}\left(P_{r, s}\right) \leq \mathbf{M}_{\theta}\left(T_{r}\right)+s, \mathbf{M}_{\theta}\left(R_{r, s}\right)<s$ and $\operatorname{spt}\left(R_{r, s}\right) \subset \mathbf{U}\left(\operatorname{spt}\left(T_{r}\right), s\right)$. In particular we have that $\partial P_{r, s}=0$. (1) and (2) hold by construction. By the lower bound on the densities there holds $\mathbf{M}\left(T_{r}\right)=\mathbf{M}_{\theta}\left(T_{r}\right)$ for almost all $r$ because slices inherit the group elements from the original chain. From now on we also assume that $2 s \leq r$.

As stated in (2.8) and (2.9), $\mathbf{M}\left(\llbracket 0 \rrbracket * T_{r}\right)=\frac{r}{m} \mathbf{M}\left(T_{r}\right)$ and also,

$$
\begin{aligned}
\mathbf{M}_{\theta}\left(\llbracket 0 \rrbracket \nVdash P_{r, s}\right)\llcorner\mathbf{B}(0, r-s)) & \leq \frac{r-s}{m} \mathbf{M}_{\theta}\left(P_{r, s}\right) \leq \frac{r}{m} \mathbf{M}_{\theta}\left(T_{r}\right)+\frac{r s}{m} \\
& =\mathbf{M}\left(\llbracket 0 \rrbracket \circledast T_{r}\right)+\frac{r s}{m} \\
& \leq \mathbf{M}\left(\llbracket 0 \rrbracket \circledast T_{r}\right)+s .
\end{aligned}
$$

Let $\bar{P}_{r, s}$ be a large enough (or infinite) scaled version of $\llbracket 0 \rrbracket \mathbb{*} P_{r, s}$ that has its boundary outside $\mathbf{U}(0,2 r)$. Since $\operatorname{spt}\left(P_{r, s}\right) \subset \mathbf{B}\left(\operatorname{spt}\left(T_{r}\right), s\right)$, we have $\operatorname{spt}\left(\llbracket 0 \rrbracket \mathbb{*} P_{r, s}\right) \subset$ 
$\operatorname{spt}\left(\bar{P}_{r, s}\right) \cap \mathbf{B}(0, r+s)$ and hence with (5.3) (note that $2 s \leq r$ ),

$$
\begin{aligned}
\max \left\{\mathbf { M } _ { \theta } \left(\bar{P}_{r, s}\llcorner\mathbf{B}(0, r))\right.\right. & \left.\mathbf{M}_{\theta}\left(\llbracket 0 \rrbracket \circledast P_{r, s}\right)\right\} \\
& \leq \mathbf{M}_{\theta}\left(\bar{P}_{r, s}\llcorner\mathbf{B}(0, r+s))\right. \\
& \leq \frac{(r+s)^{m}}{(r-s)^{m}} \mathbf{M}_{\theta}\left(\bar{P}_{r, s}\llcorner\mathbf{B}(0, r-s))\right. \\
& \leq \frac{(r+s)^{m}}{(r-s)^{m}}\left(\mathbf{M}\left(\llbracket 0 \rrbracket \circledast T_{r}\right)+s\right) .
\end{aligned}
$$

This converges to $\mathbf{M}\left(\llbracket 0 \rrbracket \mathbb{*} T_{r}\right)$ for $s \rightarrow 0$ and by replacing $s$ with a smaller value if necessary, we obtain (3).

Next we use Reifenberg's epiperimetric inequality of Theorem 3.18 to obtain a differential inequality for $f(r):=\mathbf{M}(T\llcorner\mathbf{B}(x, r))$, where $T$ is an almost minimizing $G$-chain.

5.4. Proposition. There is a constant $0<\epsilon_{5.4}(m) \leq \frac{1}{4}$ with the following property. Let $T \in \mathscr{R}_{m}(X ; G), g_{0} \in G \backslash\left\{0_{G}\right\}, 0<r_{0}<1,0<\epsilon \leq \epsilon_{5.4}(m)$ and assume that for any $0<r \leq r_{0}$ there is some $W_{r} \in \mathbf{G}(X, m)$ such that:

(1) $\operatorname{spt}(\partial T) \subset X \backslash \boldsymbol{B}\left(0,3 r_{0}\right)$,

(2) $T$ is $\left(\mathbf{M}, \xi, 2 r_{0}\right)$-minimal in $\boldsymbol{B}\left(0, r_{0}\right)$ for a continuous gauge $\xi$,

(3) $\Theta^{m}(\|T\|, x) \geq \frac{3}{4}\left\|g_{0}\right\|$ for $\|T\|$-a.e. $x \in \boldsymbol{B}\left(0, r_{0}\right)$,

(4) $d_{H}\left(\boldsymbol{B}(0,2 r) \cap \operatorname{spt}(T), \boldsymbol{B}(0,2 r) \cap W_{r}\right) \leq \epsilon r$,

(5) $\operatorname{Exc}\left(T\left\llcorner\boldsymbol{B}(0,2 r), 0, r, W_{r}\right) \leq\left\|g_{0}\right\| \epsilon r^{m}\right.$,

(6) $\pi_{W_{r} \#}\left(T\left\llcorner\left(\boldsymbol{B}(0,2 r) \cap Z_{W_{r}}(r)\right)\right)=g_{0} \llbracket \boldsymbol{B}_{W_{r}}(0, r) \rrbracket\right.$.

If we set $f(r):=\mathbf{M}\left(T\llcorner\boldsymbol{B}(0, r))\right.$ and $\lambda:=\lambda_{3.18}(m)$, then for almost every $r \in$ $\left[0, r_{0}\right]$

$$
f(r) \leq(1+\xi(r)) \frac{r}{m}\left(\lambda f^{\prime}(r)+(1-\lambda)\left\|g_{0}\right\| \boldsymbol{\alpha}(m) m r^{m-1}\right) .
$$

Proof. As in Lemma 5.3 we use the notation $T_{r}:=\partial\left(T\llcorner\mathbf{B}(0, r))\right.$ for $r \in\left[0, r_{0}\right]$. Assume that

$$
s \leq \min \left\{\left\|g_{0}\right\| \epsilon r^{m}, 2^{-1} r\right\},
$$

and for the application of Lemma 5.3 let $\theta:=\frac{3}{4}\left\|g_{0}\right\|\left(\right.$ notice $\operatorname{spt}(T) \cap \mathbf{B}\left(0, r_{0}\right)$ is compact according to Lemma 4.3) and consider the two chains $R_{r, s}$ and $P_{r, s}$ as constructed there. The constant $\theta$ is justified by our assumption on the densities of $\|T\|$. We also abbreviate $\|g\|_{\theta}:=\max \{\|g\|, \theta\}$ if $g \neq 0_{G}$ and $\mathbf{M}_{\theta}$ the associated mass. Note that $\mathbf{M} \leq \mathbf{M}_{\theta},\left\|g_{0}\right\|_{\theta}=\left\|g_{0}\right\|$ and $\mathbf{M}\left(T\llcorner B)=\mathbf{M}_{\theta}(T\llcorner B)\right.$ for every Borel set $B \subset \mathbf{B}\left(0, r_{0}\right)$. Let $\bar{P}_{r, s}$ and $\bar{T}_{r}$ be the infinite cones generated by $\llbracket 0 \rrbracket \mathbb{*} P_{r, s}$ and $\llbracket 0 \rrbracket \mathbb{*} T_{r}$ respectively. The following holds for almost all $r$ :

$$
\begin{gathered}
\operatorname{spt}\left(R_{r, s}\right) \subset \mathbf{U}\left(\operatorname{spt}\left(T_{r}\right), s\right) \subset \mathbf{U}(0, r+s) \backslash \mathbf{B}(0, r-s), \\
\operatorname{spt}\left(\llbracket 0 \rrbracket \nVdash P_{r, s}+R_{r, s}\right) \subset \mathbf{U}(0, r+s), \\
\partial\left(\llbracket 0 \rrbracket \mathbb{*} P_{r, s}+R_{r, s}\right)=T_{r}, \\
\mathbf{M}_{\theta}\left(\llbracket 0 \rrbracket \nVdash P_{r, s}\right) \leq \mathbf{M}\left(\llbracket 0 \rrbracket \mathbb{*} T_{r}\right)+s, \quad \mathbf{M}_{\theta}\left(R_{r, s}\right) \leq s, \\
\mathbf{M}_{\theta}\left(\bar{P}_{r, s}\llcorner\mathbf{B}(0, r)) \leq \mathbf{M}\left(\llbracket 0 \rrbracket \mathbb{*} T_{r}\right)+s .\right.
\end{gathered}
$$


We want to show that

$$
f(r) \leq(1+\xi(r))\left(\lambda \mathbf{M}\left(\llbracket 0 \rrbracket \mathbb{*} T_{r}\right)+(1-\lambda)\left\|g_{0}\right\| \boldsymbol{\alpha}(m) r^{m}\right),
$$

for $\lambda=\lambda_{3.18} \in\left(\frac{1}{2}, 1\right)$. In order to apply Theorem 3.18 it is necessary that the cone $\bar{T}_{r}$ has small cylindrical excess, but this may not hold for almost all $r$. So we consider two cases. First assume that $\lambda \mathbf{M}\left(\llbracket 0 \rrbracket \mathbb{*} T_{r}\right) \geq\left\|g_{0}\right\|\left(\epsilon r^{m}+\lambda \boldsymbol{\alpha}(m) r^{m}\right)$. For $W:=W_{r}$, assumptions (5) and (6) imply,

$$
\begin{aligned}
f(r) & \leq \mathbf{M}\left(T\left\llcorner\left(\mathbf{B}(0,2 r) \cap Z_{W}(r)\right)\right)\right. \\
& \leq \operatorname{Exc}\left(T\llcorner\mathbf{B}(0,2 r), 0, r, W)+\left\|g_{0}\right\| \boldsymbol{\alpha}(m) r^{m}\right. \\
& \leq\left\|g_{0}\right\|\left(\epsilon r^{m}+\boldsymbol{\alpha}(m) r^{m}\right) \\
& \leq \lambda \mathbf{M}\left(\llbracket 0 \rrbracket \mathbb{*} T_{r}\right)+(1-\lambda)\left\|g_{0}\right\| \boldsymbol{\alpha}(m) r^{m} \\
& \leq(1+\xi(r))\left(\lambda \mathbf{M}\left(\llbracket 0 \rrbracket \mathbb{*} T_{r}\right)+(1-\lambda)\left\|g_{0}\right\| \boldsymbol{\alpha}(m) r^{m}\right) .
\end{aligned}
$$

In the second case $\mathbf{M}\left(\llbracket 0 \rrbracket * T_{r}\right) \leq\left\|g_{0}\right\|\left(\lambda^{-1} \epsilon r^{m}+\boldsymbol{\alpha}(m) r^{m}\right) \leq\left\|g_{0}\right\|(2 \epsilon+\boldsymbol{\alpha}(m)) r^{m}$. By assumption (4), $\left|\pi_{W^{\perp}}(x)\right| \leq \epsilon r$ for $x \in \operatorname{spt}(T) \cap \mathbf{B}(0,2 r)$ and hence $|x| \leq(1+\epsilon) r$ for all $x \in \operatorname{spt}(T) \cap \mathbf{B}(0,2 r) \cap Z_{W}(r)$. Since we assume $s \leq\left\|g_{0}\right\| \epsilon r^{m}$, it follows from (5) and (5.8) that

$$
\begin{aligned}
\mathbf{M}_{\theta}\left(\bar{P}_{r, s}\left\llcorner Z_{W}(r)\right)\right. & \leq \mathbf{M}_{\theta}\left(\bar{P}_{r, s}\llcorner\mathbf{B}(0,(1+\epsilon) r))\right. \\
& =(1+\epsilon)^{m} \mathbf{M}_{\theta}\left(\bar{P}_{r, s}\llcorner\mathbf{B}(0, r))\right. \\
& \leq(1+\epsilon)^{m}\left(\mathbf{M}\left(\llbracket 0 \rrbracket \mathbb{X} T_{r}\right)+s\right) \\
& \leq(1+\epsilon)^{m}\left\|g_{0}\right\|\left((2 \epsilon+\boldsymbol{\alpha}(m)) r^{m}+\epsilon r^{m}\right) \\
& \leq \mathbf{c}(m)\left\|g_{0}\right\| \epsilon r^{m}+\left\|g_{0}\right\| \boldsymbol{\alpha}(m) r^{m},
\end{aligned}
$$

for some constant $\mathbf{c}(m)>0$. By the constancy theorem it is $\pi_{W \#}\left(\bar{P}_{r, s}\left\llcorner Z_{W}(r)\right)=\right.$ $g \llbracket \mathbf{B}(0, r) \rrbracket$ for some $g \in G$. It is indeed $g=g_{0}$ as we will see. From (5.6) it follows that $\partial\left(\llbracket 0 \rrbracket \nVdash P_{r, s}+R_{r, s}-(T\llcorner\mathbf{B}(0, r)))=0\right.$ and hence $\pi_{W \#}\left(\llbracket 0 \rrbracket \nVdash P_{r, s}+R_{r, s}\right)=$ $\pi_{W \#}(T\llcorner\mathbf{B}(0, r))$ by the constancy theorem. From assumptions $(4),(6)$ and the fact that $\epsilon \leq \frac{1}{4}$ it follows that the chain $\pi_{W \#}\left(T\llcorner\mathbf{B}(0, r))\right.$ has weight $g_{0}$ on $\mathbf{B}_{W}\left(0,4^{-1} r\right)$. From (5.4), (4), $s \leq \frac{r}{2}$ and $\epsilon \leq \frac{1}{4}$ it follows that

$$
\operatorname{spt}\left(\pi_{W \#} R_{r, s}\right) \subset \mathbf{B}_{W}(0, r+s) \backslash \mathbf{B}_{W}(0, r-s-\epsilon r) \subset \mathbf{B}_{W}(0, r+s) \backslash \mathbf{B}_{W}\left(0,4^{-1} r\right) .
$$

So $\pi_{W \#}\left(\llbracket 0 \rrbracket \circledast P_{r, s}\right)$ has the same weight as $\pi_{W \#}\left(T\llcorner\mathbf{B}(0, r))\right.$ on $\mathbf{B}_{W}\left(0,4^{-1} r\right)$ which is $g_{0}$. Hence, if $\mathbf{c}(m) \epsilon_{5.4}<\epsilon_{3.18}$, it follows from (5.10) that

$$
\operatorname{Exc}_{1}\left(\bar{P}_{r, s}, W\right)<\left\|g_{0}\right\| \epsilon_{3.18},
$$

and if $\epsilon_{5.4}<\frac{1}{4} \epsilon_{3.18}$, it follows from (4) and (5.4) that for all $x \in \operatorname{spt}\left(\bar{P}_{r, s}\right) \cap Z_{W}$,

$$
\left|\pi_{W^{\perp}}(x)\right| \leq \sup _{y \in \operatorname{spt}\left(T_{r}\right)} \frac{\left|\pi_{W^{\perp}}(y)\right|+s}{r-s-\left|\pi_{W^{\perp}}(y)\right|} \leq \frac{2 \epsilon_{5.4} r}{r-2 \epsilon_{5.4^{r}}} \leq 4 \epsilon_{5.4}<\epsilon_{3.18} .
$$

Hence we can apply Theorem 3.18 to the cone $\bar{P}_{r, s}$. This gives a new chain $S_{r, s} \in$ $\mathscr{R}_{m}(X ; G)$ with $\partial S_{r, s}=\partial\left(\bar{P}_{r, s}\llcorner\mathbf{B}(0, r-s))=\partial\left(\left(\llbracket 0 \rrbracket \nVdash P_{r, s}\right)\llcorner\mathbf{B}(0, r-s))\right.\right.$ and

$$
\mathbf{M}_{\theta}\left(S_{r, s}\right) \leq \lambda \mathbf{M}_{\theta}\left(\left(\llbracket 0 \rrbracket \mathbb{X} P_{r, s}\right)\llcorner\mathbf{B}(0, r-s))+(1-\lambda)\left\|g_{0}\right\| \boldsymbol{\alpha}(m)(r-s)^{m} .\right.
$$


Let $S_{r, s}^{\prime}:=S_{r, s}+R_{r, s}+\left(\llbracket 0 \rrbracket \times P_{r, s}\right)\left\llcorner\mathbf{B}(0, r-s)^{c}\right.$. By (5.6), $\partial S_{r, s}^{\prime}=T_{r}$ and by (5.7) and (5.8),

$$
\begin{aligned}
e(r, s) & :=\mathbf{M}_{\theta}\left(R_{r, s}+\left(\left(0 \circledast P_{r, s}\right)\left\llcorner\mathbf{B}(0, r-s)^{c}\right)\right)\right. \\
& \leq s+\left(\left(1+s r^{-1}\right)^{m}-\left(1-s r^{-1}\right)^{m}\right)\left(\mathbf{M}_{\theta}\left(\llbracket 0 \rrbracket \nVdash T_{r}\right)+s\right),
\end{aligned}
$$

and this converges to 0 for $s \rightarrow 0$. By the almost minimality of $T$, (5.5) and (5.7) it follows for almost all $r$ and small enough $s$,

$$
\begin{aligned}
f(r)= & \mathbf{M}\left(T\llcorner\mathbf{B}(0, r)) \leq(1+\xi(r+s)) \mathbf{M}_{\theta}\left(S_{r, s}^{\prime}\right)\right. \\
\leq & (1+\xi(r+s))\left(\mathbf{M}_{\theta}\left(S_{r, s}\right)+e(r, s)\right) \\
\leq & (1+\xi(r+s))\left(\lambda \mathbf { M } _ { \theta } \left(\left(\llbracket 0 \rrbracket \mathbb{X} P_{r, s}\right)\llcorner\mathbf{B}(0, r-s))\right.\right. \\
& \left.\quad+(1-\lambda)\left\|g_{0}\right\| \boldsymbol{\alpha}(m)(r-s)^{m}+e(r, s)\right) \\
& \quad(1+\xi(r+s))\left(\lambda\left(\mathbf{M}\left(\llbracket 0 \rrbracket \mathbb{*} T_{r}\right)+s\right)+(1-\lambda)\left\|g_{0}\right\| \boldsymbol{\alpha}(m)(r-s)^{m}+e(r, s)\right) .
\end{aligned}
$$

Taking the limit for $s \rightarrow 0$ we obtain (5.9) for almost all $r \in\left[0, r_{0}\right] . \quad f(r)=$ $\mathbf{M}(T\llcorner\mathbf{B}(0, r))$ is monotonically increasing and hence differentiable almost everywhere. By the integral slice estimate (2.7) we obtain for all $0<a \leq b \leq r_{0}$,

$$
\int_{a}^{b} \mathbf{M}\left(T_{r}\right) d r \leq \mathbf{M}(T\llcorner(\mathbf{B}(0, b) \backslash \mathbf{U}(0, a)))=f(b)-f(a) .
$$

If $r$ is a Lebesgue point of $s \mapsto \mathbf{M}\left(T_{s}\right)$ and also a point of differentiability for $f$, then $\mathbf{M}\left(T_{r}\right) \leq f^{\prime}(r)$. Almost every $r$ is such a point, hence $\mathbf{M}\left(\llbracket 0 \rrbracket \mathbb{X} T_{r}\right)=\frac{r}{m} \mathbf{M}\left(T_{r}\right) \leq$ $\frac{r}{m} f^{\prime}(r)$ for almost all $r$. Applied to formula (5.9) we obtain the result.

Next we state a lemma that treats the differential inequality obtained in Proposition 5.4.

5.5. Lemma. Let $f, \xi, \Xi:\left(0, r_{0}\right] \rightarrow[0, \infty)$ be gauges and $\lambda \in(0,1), \theta>0$. Assume that $\xi$ is continuous and for almost all $r \in\left[0, r_{0}\right]$,

$$
\begin{aligned}
\Xi(r) & =m \int_{0}^{r} \frac{\xi(t)}{t} d t<\infty, \\
\theta r^{m} & \leq \exp (\Xi(r)) f(r), \\
f(r) & \leq(1+\xi(r)) \frac{r}{m}\left(\lambda f^{\prime}(r)+(1-\lambda) \theta m r^{m-1}\right) .
\end{aligned}
$$

Assume further that $\left(1+\xi\left(r_{0}\right)\right) \sqrt{\lambda} \leq \lambda_{0}<1, \exp \left(\Xi\left(r_{0}\right)\right) \leq 2, \xi(r) \leq \Xi(r)$ and

$$
\lambda_{0} \leq \frac{\Xi(r)}{\Xi(r)+\xi(r)}, \quad \text { for all } r
$$

Then for all $0<r \leq r_{0}$,

$$
\exp (\Xi(r)) \frac{f(r)}{r^{m}}-\theta \leq \Xi(r)\left(\Xi\left(r_{0}\right)^{-1}\left(\exp \left(\Xi\left(r_{0}\right)\right) \frac{f\left(r_{0}\right)}{r_{0}^{m}}-\theta\right)+8 \theta\right) .
$$

Proof. Define the function $e(r):=\exp (\Xi(r)) f(r)-\theta r^{m}$. By assumption $e(r) \geq 0$ and w.l.o.g. we assume that $e(r)>0$ for all $r>0$. Otherwise we replace $\xi(r)$ with $\xi_{a}(r):=\xi(r)+a r$ for $a>0$. Since the final estimate is continuous in $a$ we then can take the limit for $a \downarrow 0$ afterwards. 
Since $f$ and $\Xi$ are differentiable almost everywhere, the same is true for $e$ and therefore,

$$
\begin{aligned}
e^{\prime}(r) & =\exp (\Xi(r)) f^{\prime}(r)+\Xi^{\prime}(r) \exp (\Xi(r)) f(r)-\theta m r^{m-1} \\
& =\exp (\Xi(r)) f^{\prime}(r)+m \frac{\xi(r)}{r}\left(e(r)+\theta r^{m}\right)-\theta m r^{m-1} \\
& =\exp (\Xi(r)) f^{\prime}(r)+m \frac{\xi(r)}{r} e(r)-(1-\xi(r)) \theta m r^{m-1}
\end{aligned}
$$

Hence,

$$
\begin{aligned}
e(r)= & \exp (\Xi(r)) f(r)-\theta r^{m} \\
\leq & \exp (\Xi(r))(1+\xi(r)) \frac{r}{m}\left(\lambda f^{\prime}(r)+(1-\lambda) \theta m r^{m-1}\right)-\theta r^{m} \\
= & (1+\xi(r)) \frac{\lambda r}{m}\left(e^{\prime}(r)+(1-\xi(r)) \theta m r^{m-1}-m \frac{\xi(r)}{r} e(r)\right) \\
& +\exp (\Xi(r))(1+\xi(r)) \frac{r}{m}(1-\lambda) \theta m r^{m-1}-\theta r^{m} \\
= & (1+\xi(r)) \frac{\lambda r}{m} e^{\prime}(r)-\lambda(1+\xi(r)) \xi(r) e(r) \\
& +\theta r^{m}(\exp (\Xi(r))(1+\xi(r))(1-\lambda)+(1-\xi(r))(1+\xi(r)) \lambda-1) \\
\leq & (1+\xi(r)) \frac{\lambda r}{m} e^{\prime}(r)-\lambda(1+\xi(r)) \xi(r) e(r) \\
& +\theta r^{m}(1-\lambda)(\exp (\Xi(r))(1+\xi(r))-1) .
\end{aligned}
$$

We look at two cases. First assume that for some $r$,

$$
(1-\sqrt{\lambda}) e(r) \leq \theta r^{m}(1-\lambda)(\exp (\Xi(r))(1+\xi(r))-1) .
$$

Since we assume that $\exp \left(\Xi\left(r_{0}\right)\right) \leq 2$ and $\xi(r) \leq \Xi(r)$ for all $r$, we get

$$
\begin{aligned}
e(r) & \leq \theta r^{m}(1+\sqrt{\lambda})(\exp (\Xi(r))(1+\xi(r))-1) \\
& \leq \theta r^{m}(1+\sqrt{\lambda})\left(\exp \left(\Xi\left(r_{0}\right)\right) \Xi(r)+2 \xi(r)\right) \\
& \leq 2 \theta r^{m}(2 \Xi(r)+2 \Xi(r)) \\
& \leq 8 \theta r^{m} \Xi(r)=: g(r) .
\end{aligned}
$$

For this function $g$ the derivative calculates as

$$
\begin{aligned}
g^{\prime}(r) & =8 \theta m r^{m-1} \Xi(r)+8 \theta r^{m-1} m \xi(r)=8 \theta r^{m-1} m(\Xi(r)+\xi(r)) \\
& =\frac{\Xi(r)+\xi(r)}{\Xi(r)} \frac{m}{r} g(r) .
\end{aligned}
$$

For almost all $r$ at which (5.11) doesn't hold,

$$
\sqrt{\lambda} e(r) \leq(1+\xi(r)) \frac{\lambda r}{m} e^{\prime}(r),
$$

because the sum of this inequality with the one in (5.11) has to be satisfied for almost every $r$ by our bound on $e(r)$ established before. In this case,

$$
e(r) \leq\left(1+\xi\left(r_{0}\right)\right) \frac{\sqrt{\lambda} r}{m} e^{\prime}(r) \leq \frac{\lambda_{0} r}{m} e^{\prime}(r) \leq \frac{\Xi(r)}{\Xi(r)+\xi(r)} \frac{r}{m} e^{\prime}(r) .
$$


Let $h(r):=\max \{e(r), g(r)\}$. Then for almost every $r \in\{g \geq e\}$,

$$
h^{\prime}(r)=g^{\prime}(r)=\frac{\Xi(r)+\xi(r)}{\Xi(r)} \frac{m}{r} g(r)=\frac{\Xi(r)+\xi(r)}{\Xi(r)} \frac{m}{r} h(r) .
$$

and for almost every $r \in\{g<e\}$,

$$
h^{\prime}(r)=e^{\prime}(r) \geq \frac{\Xi(r)+\xi(r)}{\Xi(r)} \frac{m}{r} e(r)=\frac{\Xi(r)+\xi(r)}{\Xi(r)} \frac{m}{r} h(r) .
$$

Hence for almost every $r$,

$$
\frac{\Xi(r)+\xi(r)}{\Xi(r)} \frac{m}{r} h(r) \leq h^{\prime}(r) .
$$

Because $\Xi^{\prime}(r)=\frac{m}{r} \xi(r)$,

$$
\begin{aligned}
\frac{\partial}{\partial r} \log \left(r^{m} \Xi(r)\right) & =\frac{\partial}{\partial r}(m \log (r)+\log (\Xi(r)))=\left(1+\frac{\xi(r)}{\Xi(r)}\right) \frac{m}{r} \\
& \leq \frac{h^{\prime}(r)}{h(r)}=\frac{\partial}{\partial r} \log (h(r)) .
\end{aligned}
$$

Integrating gives for all $0<r \leq r_{0}$,

$$
\frac{r_{0}^{m} \Xi\left(r_{0}\right)}{r^{m} \Xi(r)} \leq \frac{h\left(r_{0}\right)}{h(r)}
$$

respectively,

$$
\frac{e(r)}{r^{m}} \leq \frac{h(r)}{r^{m}} \leq \frac{\Xi(r)}{\Xi\left(r_{0}\right)} \frac{h(r)}{r_{0}^{m}} \leq \frac{\Xi(r)}{r_{0}^{m} \Xi\left(r_{0}\right)}\left(\exp \left(\Xi\left(r_{0}\right)\right) f\left(r_{0}\right)-\theta r_{0}^{m}+8 \theta r_{0}^{m} \Xi\left(r_{0}\right)\right) .
$$

This shows the lemma.

Before we proceed we give some remarks on the assumptions on $\xi$ in the lemma above.

5.6. Lemma. The following properties hold for a gauge $\xi:\left(0, r_{0}\right] \rightarrow \mathbb{R}_{+}$:

(1) If $\xi$ is concave, then $\Xi(r) \geq m \xi(r) \geq \xi(r)$ for all $r$.

(2) If $\frac{\xi(r)}{r^{\alpha}} \geq \frac{\xi(s)}{s^{\alpha}}$ for all $0<r \leq s \leq r_{0}$ and some $0<\alpha \leq 1$. Then the same holds for $\beta \in[\alpha, 1]$ in place of $\alpha$ and $\Xi(r) \geq \frac{m}{\alpha} \xi(r)$ is satisfied for all $r$. Additionally,

$$
\lambda \leq \frac{\Xi(r)}{\Xi(r)+\xi(r)} \quad \text { holds for all } r,
$$

if $\lambda \leq \frac{m}{m+\alpha}$, respectively, $\alpha \leq m \frac{1-\lambda}{\lambda}$.

Proof. If $\xi$ is concave, then for $0<r \leq r_{0}$ and $0<s \leq 1$ there holds $\xi(s r) \geq s \xi(r)$, respectively, $(s r)^{-1} \xi(s r) \geq r^{-1} \xi(r)$. This shows that

$$
\Xi(r)=m \int_{0}^{r} \frac{\xi(t)}{t} d t \geq m \int_{0}^{r} \frac{\xi(r)}{r} d t=m \xi(r) \geq \xi(r) .
$$

Let $\alpha$ and $\beta$ be as in (2), then

$$
\frac{\xi(s)}{s^{\beta}}=\frac{\xi(s)}{s^{\beta-\alpha} s^{\alpha}} \leq \frac{\xi(r)}{s^{\beta-\alpha} r^{\alpha}}=\frac{r^{\beta-\alpha}}{s^{\beta-\alpha}} \frac{\xi(r)}{r^{\beta}} \leq \frac{\xi(r)}{r^{\beta}} .
$$


Similarly to the concave case,

$$
\begin{aligned}
\Xi(r) & =m \int_{0}^{r} \frac{\xi(t)}{t} d t \geq m \int_{0}^{r} t^{\alpha-1} \frac{\xi(r)}{r^{\alpha}} d t \\
& =\frac{m}{\alpha} \xi(r) .
\end{aligned}
$$

For all $r$ we get

$$
\frac{\Xi(r)}{\Xi(r)+\xi(r)} \geq \frac{\Xi(r)}{\Xi(r)+\frac{\alpha}{m} \Xi(r)}=\frac{m}{m+\alpha} .
$$

5.2. Upper excess bounds and Reifenberg flatness. First we give conditions that allow to estimate the excess in a neighborhood of a point with small excess.

5.7. Lemma. Let $\phi$ be a finite Borel measure on $X, 0<r_{0}, \epsilon, \eta \leq 1, \xi:\left(0,2 r_{0}\right] \rightarrow$ $(0, \epsilon]$ be a gauge and $|x| \leq r_{0} \eta$. Assume that

(1) $\Theta^{m}(\phi, 0)=\Theta^{m}(\phi, x)=1$,

(2) $\operatorname{exc}^{m}\left(\phi, 0,2 r_{0}\right) \leq 1$,

(3) for $0<s \leq t \leq r_{0}$ and $y \in\{0, x\}$,

$$
\exp (\xi(s)) \frac{\phi(\boldsymbol{B}(y, s))}{\boldsymbol{\alpha}(m) s^{m}} \leq \exp (\xi(t)) \frac{\phi(\boldsymbol{B}(y, t))}{\boldsymbol{\alpha}(m) t^{m}} .
$$

Then

$$
\operatorname{exc}^{m}\left(\phi, x, r_{0}\right) \leq \operatorname{exc}^{m}\left(\phi, 0, r_{0}(1+\eta)\right)+2^{m+6} \max \{\eta, \epsilon\} .
$$

Proof. Because of (1) and (2), the following estimate holds for all $0<r \leq 2 r_{0}$,

$$
\frac{\phi(\mathbf{B}(0, r))}{\boldsymbol{\alpha}(m) r^{m}} \leq \operatorname{exc}^{m}\left(\phi, 0,2 r_{0}\right)+1 \leq 2 .
$$

Let $0<r \leq r_{0}$ be such that $|x|=\eta r$ (if $|x|=0$ there is nothing to show). We first assume that the functions in (3) are strictly increasing on $\left(0, r_{0}\right]$. Clearly,

$$
\begin{aligned}
\frac{\phi(\mathbf{B}(x, r))}{\boldsymbol{\alpha}(m) r^{m}} & \leq \frac{\phi(\mathbf{B}(0,|x|+r))}{\boldsymbol{\alpha}(m)(|x|+r)^{m}} \frac{(|x|+r)^{m}}{r^{m}} \\
& =\frac{\phi(\mathbf{B}(0, r(1+\eta)))}{\boldsymbol{\alpha}(m)(r(1+\eta))^{m}}(1+\eta)^{m}
\end{aligned}
$$

Hence for $0<t \leq r$,

$$
\begin{aligned}
\exp (\xi(t)) \frac{\phi(\mathbf{B}(x, t))}{\boldsymbol{\alpha}(m) t^{m}} & \leq \exp (\xi(r)) \frac{\phi(\mathbf{B}(x, r))}{\boldsymbol{\alpha}(m) r^{m}} \\
& \leq \exp (\xi(r))(1+\eta)^{m} \frac{\phi(\mathbf{B}(0, r(1+\eta)))}{\boldsymbol{\alpha}(m)(r(1+\eta))^{m}}
\end{aligned}
$$

Since

$$
\lim _{s \downarrow 0} \exp (\xi(s)) \frac{\phi(\mathbf{B}(x, s))}{\boldsymbol{\alpha}(m) s^{m}}=\lim _{s \downarrow 0} \exp (\xi(s)) \frac{\phi(\mathbf{B}(0, s))}{\boldsymbol{\alpha}(m) s^{m}},
$$

and these functions are strictly increasing, there is for any $s \in(0, r]$ some $s^{\prime} \in(0, s]$ with

$$
\exp \left(\xi\left(s^{\prime}\right)\right) \frac{\phi\left(\mathbf{B}\left(0, s^{\prime}\right)\right)}{\boldsymbol{\alpha}(m) s^{\prime m}} \leq \exp (\xi(s)) \frac{\phi(\mathbf{B}(x, s))}{\boldsymbol{\alpha}(m) s^{m}} .
$$


Thus,

$$
-\frac{\phi(\mathbf{B}(x, s))}{\boldsymbol{\alpha}(m) s^{m}} \leq-\exp \left(\xi\left(s^{\prime}\right)-\xi(s)\right) \frac{\phi\left(\mathbf{B}\left(0, s^{\prime}\right)\right)}{\boldsymbol{\alpha}(m) s^{\prime m}} \leq-\exp (-\xi(s)) \frac{\phi\left(\mathbf{B}\left(0, s^{\prime}\right)\right)}{\boldsymbol{\alpha}(m) s^{\prime m}} .
$$

If we assume that $\exp (\xi(r))(1+\eta)^{m} \leq 1+\delta$ and $\exp (-\xi(r)) \geq 1-\delta$ for some $\delta>0$, and combine (5.14), (5.15) with (5.16), we get for $s, t \in(0, r]$,

$$
\begin{aligned}
\frac{\phi(\mathbf{B}(x, t))}{\boldsymbol{\alpha}(m) t^{m}}-\frac{\phi(\mathbf{B}(x, s))}{\boldsymbol{\alpha}(m) s^{m}} & \leq(1+\delta) \frac{\phi(\mathbf{B}(0, r(1+\eta)))}{\boldsymbol{\alpha}(m)(r(1+\eta))^{m}}-(1-\delta) \frac{\phi\left(\mathbf{B}\left(0, s^{\prime}\right)\right)}{\boldsymbol{\alpha}(m) s^{\prime m}} \\
& \leq \mathbf{e x c}^{m}(\phi, 0, r(1+\eta))+4 \delta .
\end{aligned}
$$

Since $\eta \leq 1$ and $\xi\left(2 r_{0}\right) \leq \epsilon \leq 1$, there holds

$$
\begin{aligned}
\exp (\xi(r))(1+\eta)^{m}-1 & \leq(1+4 \epsilon)\left(1+2^{m} \eta\right)-1 \\
& \leq\left(4+2^{m}+2^{m+2}\right) \max \{\epsilon, \eta\} \\
& \leq 2^{m+4} \max \{\epsilon, \eta\},
\end{aligned}
$$

and similarly, $\exp (-\xi(r)) \geq 1-\epsilon$. Hence

$$
\operatorname{exc}^{m}(\phi, x, r) \leq \operatorname{exc}^{m}(\phi, 0, r(1+\eta))+2^{m+6} \max \{\epsilon, \eta\} .
$$

This shows the lemma assuming that $s \mapsto \exp (\xi(s)) \frac{\phi(\mathbf{B}(x, s))}{\boldsymbol{\alpha}(m) s^{m}}$ is strictly increasing. The general case follows by replacing $\xi(s)$ with $\xi(s)+a s$ and taking the limit $a \downarrow 0$.

The following bootstrap argument is an application of the moments computations. It is the key lemma for showing Reifenberg flatness of the support of a chain in a neighborhood of small excess.

5.8. Lemma. Let $T \in \mathscr{R}_{m}(X ; G)$ and $0<r_{0}, s_{0}, \epsilon \leq 1$ be such that:

(1) $0<4 \sqrt{s_{0}} \leq r_{0} \leq \epsilon \leq \frac{1}{81}$.

(2) $\operatorname{spt}(\partial T) \subset X \backslash \boldsymbol{B}\left(0,2 r_{0}\right)$.

(3) $\Theta^{m}(\|T\|, x)=\theta>0$ for $\|T\|$-almost all $x \in \operatorname{spt}(T) \cap \boldsymbol{B}\left(0,2 r_{0}\right)$.

(4) There is a plane $W \in \mathbf{G}(X ; G)$ and $g_{0} \in G$ with $\left\|g_{0}\right\|=\Theta^{m}(\|T\|, 0)=\theta$, such that $\lim _{r \downarrow 0} \beta_{\infty}(\|T\|, 0, r, W)=0$ and for all sufficiently small $r$, $\pi_{W \#}\left(T\left\llcorner\left(\boldsymbol{B}(x, r) \cap Z_{W}\left(\pi_{W}(x), 2^{-1} r\right)\right)\right)=g_{0} \llbracket \boldsymbol{B}_{W}\left(\pi_{W}(x), 2^{-1} r\right) \rrbracket\right.$.

(5) there holds,

$$
\begin{aligned}
\mathbf{e x c}_{*}^{m}\left(\|T\|, x, r_{0}\right) & \leq \theta \epsilon \text { for all } x \in \boldsymbol{B}\left(0,2 r_{0}\right), \\
\mathbf{e x c}^{m *}\left(\|T\|, x, r_{0}\right) & \leq \theta \epsilon \text { for }\|T\|-\text { almost every } x \in \boldsymbol{B}\left(0,2 r_{0} \epsilon^{\frac{1}{4}}\right) .
\end{aligned}
$$

If

$$
\mathbf{c}_{4.15}(m) \epsilon^{\frac{1}{4(m+2)}} \leq \frac{1}{25 \sqrt{m}}
$$

then for all $r \in\left(0, s_{0}\right)$ there is a plane $W_{r} \in \mathbf{G}(X, m)$ such that

$$
d_{H}\left(\operatorname{spt}(T) \cap \boldsymbol{B}(0, r), W_{r} \cap \boldsymbol{B}(0, r)\right)<2 \mathbf{c}_{4.15}(m) r \epsilon^{\frac{1}{4(m+2)}} .
$$

and

$$
\pi_{W_{r} \#}\left(T\left\llcorner\boldsymbol{B}(0, r) \cap Z_{W_{r}}\left(0, \frac{r}{2}\right)\right)=g_{0} \llbracket \boldsymbol{B}_{W_{r}}\left(0, \frac{r}{2}\right) \rrbracket .\right.
$$

Further, for any $r \in\left(0, s_{0}\right)$ there is an orthogonal frame $x_{1}, \ldots, x_{m} \in \operatorname{spt}(T) \cap$ $\partial \boldsymbol{B}(0, r)$ with $x_{i} \perp x_{j}$ for $i \neq j$. 
THIERRY DE PAUW AND ROGER ZÜST

Proof. Let $\phi:=\theta^{-1}\|T\|$. By assumption, $s_{0}$ satisfies the bounds of Corollary 4.15. Consider the sequence $s_{k}$ defined by $s_{k+1}:=2 s_{k} \epsilon^{\frac{1}{4}}$. The factor 2 in this definition is to compensate for the fact that the $\boldsymbol{\beta}_{\infty}$ estimate in Corollary 4.15 is at scale $1 / 2$. This is a monotone sequence with $\lim _{k \rightarrow \infty} s_{k}=0$ since by definition

$$
s_{k+1} \leq 2 s_{k} \epsilon^{\frac{1}{4}} \leq \frac{2}{3} s_{k} .
$$

Because of (4) there is some $k \geq 1$ such that for any $s \in\left(0, s_{k}\right)$,

$$
\beta_{\infty}(\phi, 0, s, W)<\frac{1}{25 \sqrt{m}},
$$

and

$$
\pi_{W \#}\left(T\left\llcorner\left(\mathbf{B}(0, s) \cap Z_{W}\left(0, \frac{s}{2}\right)\right)\right)=g_{0} \llbracket \mathbf{B}_{W}\left(0, \frac{s}{2}\right) \rrbracket .\right.
$$

From Lemma 4.13 we obtain that for all $s \in\left(0, s_{k}\right)$ there is an orthogonal frame $x_{1}(s), \ldots, x_{n}(s) \in X$ such that

$$
x_{i}(s) \in \operatorname{spt}(T), \quad\left|x_{i}(s)\right|=s \quad \text { and } \quad x_{i}(s) \perp x_{j}(s) \text { if } i \neq j .
$$

For any $r \in\left(0, s_{k-1}\right)$ set $s:=2 r \epsilon^{\frac{1}{4}}<s_{k}$. Corollary 4.15 implies that for all but countably many $r$ (i.e. those $r$ with $\|T\|(\partial \mathbf{B}(0, r))=0)$ there is some plane $W_{r} \in \mathbf{G}(X, m)$ with

$$
\beta_{\infty}\left(\|T\|, 0, r, W_{r}\right)<\mathbf{c}_{4.15}(m) \epsilon^{\frac{1}{4(m+2)}} \leq \frac{1}{25 \sqrt{m}} .
$$

More precisely, the plane $W_{r}$ is spanned by the vectors $x_{i}(s), i=1, \ldots, m$, from (5.19) and because of hypothesis (3) we can approximate $x_{i}(s)$ as necessary for Corollary 4.15. In order to proceed the argument we show that

$$
\pi_{W_{r} \#}\left(T\left\llcorner\left(\mathbf{B}(0, r) \cap Z_{W_{r}}\left(0, \frac{r}{2}\right)\right)\right)=g_{0} \llbracket \mathbf{B}_{W_{r}}\left(0, \frac{r}{2}\right) \rrbracket .\right.
$$

This is achieved with Lemma 2.7 by estimating the distance from $W_{r}$ to $W$. Since $x_{i}(s) \in \operatorname{spt}(T) \cap \mathbf{B}(0, s)$ by (5.19), we obtain from (5.20),

$$
\left|\pi_{W}\left(x_{i}(s)\right)-x_{i}(s)\right|=\left|\pi_{W^{\perp}}\left(x_{i}(s)\right)\right| \leq \beta_{\infty}(\|T\|, 0, s, W) s<\frac{s}{25 \sqrt{m}} .
$$

For all $w \in W_{r}$, if we write $w=\sum_{i} \lambda_{i} x_{i}(s)$ with $|w|^{2}=s^{2} \sum_{i}\left|\lambda_{i}\right|^{2}$,

$$
\left|\pi_{W}(w)-w\right|<\frac{s}{25 \sqrt{m}} \sum_{i}\left|\lambda_{i}\right| \leq \frac{|w|}{25} .
$$

Hence $\left\|\pi_{W}-\pi_{W_{r}}\right\| \leq \frac{1}{5}$ from Lemma 2.1 and Lemma 2.7 implies together with (5.18) that (5.21) holds for $W_{r}$ if $r \in\left(0, s_{k-1}\right)$ and $\|T\|(\partial \mathbf{B}(0, r))=0$. Because the inequality in (5.20) is strict, (5.20) and (5.21) hold for all $r \in\left(0, s_{k-1}\right)$. Again from Lemma 4.13 it follows that we can find an orthogonal frame as in (5.19) for all $r \in\left(0, s_{k-1}\right)$.

Proceeding this way we conclude by induction on $k$ that for all $r \in\left(0, s_{0}\right)$ there is such a plane $W_{r} \in \mathbf{G}(X, m)$. To obtain the estimate on the Hausdorff distance note that for all $x \in \operatorname{spt}(T) \cap \mathbf{B}(0, r)$,

$$
\operatorname{dist}\left(x, W_{r} \cap \mathbf{B}(0, r)\right)<\mathbf{c}_{4.15}(m) r \epsilon^{\frac{1}{4(m+2)}} .
$$

On the other side, (5.21) implies that for any

$$
x \in W_{r} \cap \mathbf{B}\left(0, r\left(1-\mathbf{c}_{4.15}(m) \epsilon^{\frac{1}{4(m+2)}}\right)\right)
$$




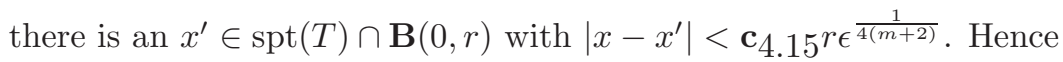

$$
d_{\mathrm{H}}\left(\operatorname{spt}(T) \cap \mathbf{B}(0, r), W_{r} \cap \mathbf{B}(0, r)\right)<2 \mathbf{c}_{4.15}(m) r \epsilon^{\frac{1}{4(m+2)}} .
$$

The following result shows that almost monotonic chains have neighborhoods that are Reifenberg flat. The assumption on the almost constant densities will be justified below in Lemma 5.11 for nearly monotonic chains if we have a discreteness assumption on $G$. Moreover an almost monotonic chain is nearly monotonic because of Lemma 4.2 .

5.9. Theorem. Let $T \in \mathscr{R}_{m}(X ; G)$ and assume that there are $x_{0} \in X, \theta>0, r_{0} \in$ $(0,1]$ with

(1) $\operatorname{spt}(\partial T) \subset X \backslash \boldsymbol{B}\left(x_{0}, 2 r_{0}\right)$,

(2) $\Theta^{m}(\|T\|, x)=\Theta^{m}\left(\|T\|, x_{0}\right)=\theta$ for $\|T\|$-almost every $x \in \boldsymbol{B}\left(x_{0}, r_{0}\right)$,

(3) $T$ is almost monotonic in $\boldsymbol{B}\left(x_{0}, r_{0}\right)$ for some gauge $\xi:\left(0, r_{0}\right] \rightarrow(0, \infty)$.

Then for any $\epsilon>0$ there is a $r_{\epsilon} \in\left(0, r_{0}\right]$ such that for all $x \in \operatorname{spt}(T) \cap \boldsymbol{B}\left(x_{0}, r_{\epsilon}\right)$ and all $r \in\left(0, r_{\epsilon}\right]$ there is a plane $W \in \mathbf{G}(X, m)$ and $g_{x} \in G$ with the properties,

$$
\begin{aligned}
& \operatorname{exc}^{m}\left(\|T\|, x, r_{\epsilon}\right) \leq \theta \epsilon, \\
& d_{H}(\operatorname{spt}(T) \cap \boldsymbol{B}(x, r),(x+W) \cap \boldsymbol{B}(x, r)) \leq r \epsilon, \\
& \pi_{W \#}\left(T \left\llcorner\left(\boldsymbol{B}(x, r) \cap Z_{W}\left(\pi_{W}(x), \frac{r}{2}\right)\right)=g_{x} \llbracket \boldsymbol{B}_{W}\left(\pi_{W}(x), \frac{r}{2}\right) \rrbracket,\right.\right.
\end{aligned}
$$

and $\left\|g_{x}\right\|=\theta$. Further, for any $x \in \operatorname{spt}(T) \cap \boldsymbol{B}\left(x_{0}, r_{\epsilon}\right)$ and $r \in\left(0, r_{\epsilon}\right]$ there is an orthogonal frame $x_{1}, \ldots, x_{m}$ with $\left|x_{i}\right|=r$ and $x+x_{i} \in \operatorname{spt}(T)$.

Proof. Fix some $\epsilon>0$ with

$$
\epsilon \leq \frac{1}{2^{m+7}} \min \left\{\frac{1}{81}, \frac{1}{\left(25 \sqrt{m} \mathbf{c}_{4.15}(m)\right)^{4(m+2)}}\right\} .
$$

The finite Borel measure $\phi:=\theta^{-1}\|T\|$ is almost monotonic in $\mathbf{B}\left(x_{0}, r_{0}\right)$ with respect to the same gauge as $\|T\|$. From Lemma 4.2 it follows that $\mathbf{e x c}_{*}^{m}(\|T\|, x, r) \leq$ $\mathbf{c}_{4.2} \xi(r)$ for all $x \in \mathbf{B}\left(x_{0}, r_{0}\right)$ and $0<r \leq r_{0}$. Since $\Theta^{m}\left(\phi, x_{0}\right)=1$ there is a $r_{1} \in\left(0, \frac{r_{0}}{6}\right]$ such that

$$
\max \left\{\theta^{-1} \mathbf{c}_{4.2} \xi\left(6 r_{1}\right), \operatorname{exc}^{m}\left(\phi, x_{0}, 6 r_{1}\right)\right\} \leq \epsilon .
$$

According to Lemma 5.7, any $x \in \mathbf{B}\left(x_{0}, 3 r_{1} \epsilon\right)$ with $\Theta^{m}(\phi, x)=1$ satisfies

$$
\begin{aligned}
\operatorname{exc}^{m}\left(\phi, x, 3 r_{1}\right) & \leq \operatorname{exc}^{m}\left(\phi, x_{0}, 6 r_{1}\right)+2^{m+6} \max \{\epsilon, \epsilon\} \leq 2^{m+7} \epsilon \\
& \leq \min \left\{\frac{1}{81}, \frac{1}{\left(25 \sqrt{m} \mathbf{c}_{4.15}(m)\right)^{4(m+2)}}\right\} .
\end{aligned}
$$

Let $r_{2}:=\frac{1}{16}\left(r_{1} \epsilon\right)^{2}$, respectively, $4 \sqrt{r_{2}} \leq r_{1} \epsilon$. Since $\mathbf{c}_{4.2} \xi\left(6 r_{1}\right) \leq \frac{\theta}{2}$, it follows from Lemma 4.3, that $\|T\|$ is Ahlfors-regular in $\mathbf{U}\left(x_{0}, 6 r_{1}\right)$ and hence tangent planes for $T$ exist for $\|T\|$-a.e. $x \in \mathbf{U}\left(x_{0}, 6 r_{1}\right)$ by Lemma 2.6. Consider a point $x \in \mathbf{B}\left(x_{0}, r_{2}\right)$ with $\Theta^{m}(\|T\|, x)=\theta$ at which such a tangent plane exists. Note that $\mathbf{B}\left(x, 2 r_{1} \epsilon\right) \subset$ $\mathbf{B}\left(x_{0}, 3 r_{1} \epsilon\right)$ and $\max \left\{\mathbf{e x c}^{m *}(\phi, y, r), \mathbf{e x c}_{*}^{m}(\phi, z, r)\right\} \leq 2^{m+7} \epsilon$ if $0<4 \sqrt{r} \leq r_{1} \epsilon$, $y \in \mathbf{B}\left(x, 2 r_{1} \epsilon\right)$ and $z \in \mathbf{B}\left(x, 2 r_{1} \epsilon\right)$ with $\Theta^{m}(\phi, x)=1$. From Lemma 5.8 and (5.22) we obtain that for any $0<r<r_{2}$ there is a plane $W_{x, r} \in \mathbf{G}(X, m)$ such that,

$$
d_{\mathrm{H}}\left(\operatorname{spt}(T) \cap \mathbf{B}(x, r), W_{x, r} \cap \mathbf{B}(x, r)\right)<2 \mathbf{c}_{4.15}(m) r\left(2^{m+7} \epsilon\right)^{\frac{1}{4(m+2)}} \leq \frac{2 r}{25} .
$$


and

$$
\pi_{W_{x, r} \#}\left(T \left\llcorner\left(\mathbf{B}(x, r) \cap Z_{W_{x, r}}\left(\pi_{W_{x, r}}(x), \frac{r}{2}\right)\right)=g_{x} \llbracket \mathbf{B}_{W_{x, r}}\left(x, \frac{r}{2}\right) \rrbracket .\right.\right.
$$

with $\left\|g_{x}\right\|=\theta$. Moreover, there is an orthogonal frame centered at $x$ for all scales $0<r<r_{2}$ as described in the theorem. The set of those points $x \in \operatorname{spt}(T) \cap$ $\mathbf{U}\left(x_{0}, r_{2}\right)$ at which tangent planes exist and $\Theta^{m}(\|T\|, x)=\theta$ is dense. Thus for all $x \in \mathbf{U}\left(x_{0}, r_{2}\right) \cap \operatorname{spt}(T)$ and all $0<r<r_{2}$ there is a $W_{x, r} \in \mathbf{G}(X, m)$ such that (5.23) holds as well as (5.24) for some $g_{x, r} \in G$ with $\left\|g_{x, r}\right\|=\theta$. $g_{x, r}$ does not depend on $r$ because of Corollary 2.4 and Lemma 2.7. Lemma 4.3 and Lemma 4.2 imply that $\operatorname{spt}(T) \cap \mathbf{B}(x, r)$ is compact if $x \in \mathbf{U}\left(x_{0}, r_{2}\right)$ and $r$ is small enough. Thus the statement about the orthogonal frames holds for all points in $\operatorname{spt}(T) \cap \mathbf{U}\left(x_{0}, r_{2}\right)$. Let $x \in \operatorname{spt}(T) \cap \mathbf{U}\left(x_{0}, r_{2}\right)$ and $x_{i}$ be a sequence in $\mathbf{U}\left(x_{0}, r_{2}\right)$ with $\Theta^{m}(\|T\|, x)=\theta$ that converges to $x_{0}$. Let $0<r<3 r_{1}$ and $\lambda>1$ with $\lambda r<3 r_{1}$. Applying (5.22) to the sequence $x_{i}$,

$$
\begin{aligned}
\frac{\|T\|(\mathbf{B}(x, r))}{\boldsymbol{\alpha}(m) r^{m}}-\theta & \leq \limsup _{i \rightarrow \infty} \frac{\|T\|\left(\mathbf{B}\left(x_{i}, \lambda r\right)\right)}{\boldsymbol{\alpha}(m) r^{m}}-\theta \\
& \leq \limsup _{i \rightarrow \infty}\left|\frac{\|T\|\left(\mathbf{B}\left(x_{i}, \lambda r\right)\right)}{\boldsymbol{\alpha}(m)(\lambda r)^{m}}-\theta\right| \lambda^{m}+\left|\theta \lambda^{m}-\theta\right| \\
& \leq \mathbf{e x c}^{m}\left(\|T\|, x, 3 r_{1}\right)+\left(\lambda^{m}-1\right) \theta \\
& \leq \theta 2^{m+7} \epsilon \lambda^{m}+\left(\lambda^{m}-1\right) \theta .
\end{aligned}
$$

Similarly we obtain a lower bound of $-\theta 2^{m+7} \epsilon \lambda^{-m}+\left(\lambda^{-m}-1\right) \theta$. Taking the limit for $\lambda \downarrow 1$, it follows that $\operatorname{exc}^{m}(\|T\|, x, r) \leq \theta 2^{m+8} \epsilon$ for all $x \in \operatorname{spt}(T) \cap \mathbf{U}\left(x_{0}, r_{2}\right)$ and $0<r<3 r_{1}$. This concludes the proof.

5.3. Main regularity results. Combining Theorem 5.9, Lemma 4.2, Lemma 5.1, Proposition 5.4, Lemma 5.5 and Lemma 5.6 we obtain.

5.10. Proposition. Assume that $T \in \mathscr{R}_{m}(X ; G), x_{0} \in \operatorname{spt}(T)$ and $r_{0}>0$ satisfy:

(1) $\operatorname{spt}(\partial T) \subset X \backslash \boldsymbol{B}\left(x_{0}, 2 r_{0}\right)$.

(2) $T$ is $\left(\mathbf{M}, \xi, r_{0}\right)$-minimal in $\boldsymbol{B}\left(x_{0}, r_{0}\right)$ for a continuous gauge $\xi$ with $\Xi(\delta)=$ $m \int_{0}^{r_{0}} \frac{\xi(s)}{s} d s<\infty$

(3) $\Theta^{m}(\|T\|, x)=\Theta^{m}\left(\|T\|, x_{0}\right)=\theta$ for $\|T\|$-almost every $x \in \boldsymbol{B}\left(x_{0}, r_{0}\right)$.

(4) Set $\lambda_{0}:=\sqrt[4]{\lambda_{3.18}}$. Assume that $\left(1+\xi\left(r_{0}\right)\right) \lambda_{0} \leq 1$, $\exp \left(\Xi\left(r_{0}\right)\right) \leq 2$ and $s \mapsto \frac{\xi(s)}{s^{\alpha}}$ is decreasing for some $\alpha \leq m \frac{1-\lambda_{0}}{\lambda_{0}}$.

Then there are constants $r_{1}>0$ and $\mathbf{c}_{5.10}\left(m, r_{0}, \Xi\left(r_{0}\right), \theta, \mathbf{M}(T)\right)>0$ such that for all $0<r \leq r_{1}$,

$$
\begin{aligned}
\exp (\Xi(r)) \frac{\|T\|(\boldsymbol{B}(x, r))}{\boldsymbol{\alpha}(m) r^{m}}-\theta \text { is increasing in } r \text { if } x \in \boldsymbol{U}\left(x_{0}, r_{1}\right), \\
\mathbf{e x c}_{*}^{m}(\|T\|, x, r) \leq \mathbf{c}_{5.10} \Xi(r), \text { if } x \in \boldsymbol{U}\left(x_{0}, r_{1}\right), \\
\exp (\Xi(r)) \frac{\|T\|(\boldsymbol{B}(x, r))}{\boldsymbol{\alpha}(m) r^{m}}-\theta \leq \mathbf{c}_{5.10} \Xi(r), \text { if } x \in \operatorname{spt}(T) \cap \boldsymbol{U}\left(x_{0}, r_{1}\right), \\
\mathbf{e x c}^{m *}(\|T\|, x, r) \leq \mathbf{c}_{5.10} \Xi(r), \text { if } x \in \operatorname{spt}(T) \cap \boldsymbol{U}\left(x_{0}, r_{1}\right) .
\end{aligned}
$$

In particular $\Theta^{m}(\|T\|, x)=\theta$ for all $x \in \operatorname{spt}(T) \cap \boldsymbol{U}\left(x_{0}, r_{1}\right)$.

Proof. From Lemma 5.1 it follows that $T$ is almost monotonic in $\mathbf{B}\left(x_{0}, r_{0}\right)$ with gauge $\Xi:\left(0, r_{0}\right] \rightarrow \mathbb{R}_{+}$. From Lemma 4.2 it follows that $\|T\|$ is nearly monotonic in 
$\mathbf{B}\left(x_{0}, r_{0}\right)$ with some gauge $\mathbf{c}_{*} \Xi$ where $\mathbf{c}_{*}:=\mathbf{c}_{4.2}\left(m, r_{0}, \Xi\left(r_{0}\right), \mathbf{M}(T)\right)$. This shows the first two conclusions.

From Theorem 5.9 it follows that for any $0<\epsilon<1$ there is a scale $0<r_{\epsilon} \leq \frac{r_{0}}{2}$ such that for all $x \in \operatorname{spt}(T) \cap \mathbf{B}\left(x_{0}, r_{\epsilon}\right)$ and $0<r \leq r_{\epsilon}$ there is a plane $W=W_{x, r} \in$ $\mathbf{G}(X, m)$ and $g_{x} \in G$ with $\left\|g_{x}\right\|=\theta$ and

$$
\begin{gathered}
\operatorname{exc}^{m}\left(\|T\|, x, 2 r_{\epsilon}\right) \leq \theta \epsilon, \\
d_{\mathrm{H}}(\operatorname{spt}(T) \cap \mathbf{B}(x, 2 r),(x+W) \cap \mathbf{B}(x, 2 r)) \leq r \epsilon, \\
\pi_{W \#}\left(T \left\llcorner\left(\mathbf{B}\left(\pi_{W}(x), 2 r\right) \cap Z_{W}\left(\pi_{W}(x), r\right)\right)=g_{x} \llbracket \mathbf{B}_{W}\left(\pi_{W}(x), r\right) \rrbracket .\right.\right.
\end{gathered}
$$

In order to apply Proposition 5.4 we need to check that $T$ has small cylindrical excess over $W$. But this is rather simple using the two properties above. Let $x \in \mathbf{B}\left(0, r_{\epsilon}\right)$. For simplicity we translate the chain to the origin $T^{\prime}:=\tau_{-x \#} T$, where $\tau_{-x}(y)=y-x$. Then for any $y \in \operatorname{spt}\left(T^{\prime}\right) \cap \mathbf{B}(0,2 r) \cap Z_{W}(0, r)$ there holds $|y| \leq r+r \epsilon$ and thus

$$
\operatorname{spt}\left(T^{\prime}\right) \cap \mathbf{B}(0,2 r) \cap Z_{W}(0, r) \subset \mathbf{B}(0, r(1+\epsilon)) .
$$

Therefore,

$$
\begin{aligned}
\frac{1}{\boldsymbol{\alpha}(m) r^{m}} & \operatorname{Exc}\left(T^{\prime}\llcorner\mathbf{B}(0,2 r), 0, r, W)\right. \\
& =\frac{1}{\boldsymbol{\alpha}(m) r^{m}}\left(\left\|T^{\prime}\right\|\left(\mathbf{B}(0,2 r) \cap Z_{W}(r)\right)-\theta \boldsymbol{\alpha}(m) r^{m}\right) \\
& \leq \frac{1}{\boldsymbol{\alpha}(m) r^{m}}\left(\left\|T^{\prime}\right\|(\mathbf{B}(0, r(1+\epsilon)))-\theta \boldsymbol{\alpha}(m) r^{m}\right) \\
& =\frac{\left\|T^{\prime}\right\|(\mathbf{B}(0, r(1+\epsilon)))}{\boldsymbol{\alpha}(m)(1+\epsilon)^{m} r^{m}}(1+\epsilon)^{m}-\theta \\
& \leq\left(\theta+\mathbf{e x c}^{m}\left(\left\|T^{\prime}\right\|, 0,2 r_{\epsilon}\right)\right)(1+\epsilon)^{m}-\theta \\
& \leq(\theta+\epsilon \theta)\left(1+2^{m} \epsilon\right)-\theta \\
& \leq \theta\left(2^{m}+2^{m} \epsilon+1\right) \epsilon .
\end{aligned}
$$

So if $\epsilon$ is small enough we can apply Proposition 5.4. It follows that for all $x \in$ $\mathbf{B}\left(x_{0}, r_{\epsilon}\right)$ with $\Theta^{m}(\|T\|, x)=\theta$ and for almost all $0<r \leq r_{\epsilon}$,

$$
f_{x}(r) \leq(1+\xi(r)) \frac{r}{m}\left(\lambda f_{x}^{\prime}(r)+(1-\lambda) \theta \boldsymbol{\alpha}(m) m r^{m-1}\right),
$$

where $f_{x}(r):=\|T\|(\mathbf{B}(x, r))$ and $0<\lambda:=\lambda_{3.18}<1$. Note that (4) implies that $\left(1+\xi\left(r_{0}\right)\right) \sqrt{\lambda_{3.18}} \leq \lambda_{0}=\sqrt[4]{\lambda_{3.18}}$. Hence with the help of Lemma 5.6 we can apply Lemma 5.5 and obtain

$$
\frac{f_{x}(r)}{\boldsymbol{\alpha}(m) r^{m}}-\theta \leq \exp (\Xi(r)) \frac{f_{x}(r)}{\boldsymbol{\alpha}(m) r^{m}}-\theta \leq \mathbf{c}^{*} \Xi(r),
$$

for all $0<r \leq r_{\epsilon}$ and some $\mathbf{c}^{*}=\mathbf{c}^{*}\left(m, r_{0}, \Xi\left(r_{0}\right), \theta, \mathbf{M}(T)\right)$. Accordingly, $\operatorname{exc}^{m *}(\|T\|, x, r) \leq\left(\mathbf{c}^{*}+\mathbf{c}_{*}\right) \Xi(r)$ if $x \in \mathbf{B}\left(x_{0}, r_{\epsilon}\right)$ satisfies $\Theta^{m}(\|T\|, x)=\theta$ and $0<r \leq r_{\epsilon}$.

Now assume that $x \in \operatorname{spt}(T) \cap \mathbf{U}\left(x_{0}, r_{\epsilon}\right)$ and let $0<r<r_{\epsilon}$. Let $x_{k}$ be a sequence in $\mathbf{B}\left(x_{0}, r_{\epsilon}\right)$ with $\left|x_{k}-x\right|=r \delta_{k} \rightarrow 0$ and $\Theta^{m}\left(\|T\|, x_{k}\right)=\theta$ for all $k$. Since $\Xi$ is 
continuous,

$$
\begin{aligned}
\mathbf{c}^{*} \Xi(r) \geq \frac{\|T\|(\mathbf{B}(x, r))}{\boldsymbol{\alpha}(m) r^{m}}-\theta & =\left(1+\delta_{k}\right)^{m} \frac{\|T\|(\mathbf{B}(x, r))}{\boldsymbol{\alpha}(m) r^{m}\left(1+\delta_{k}\right)^{m}}-\theta \\
& \leq\left(1+\delta_{k}\right)^{m} \frac{\|T\|\left(\mathbf{B}\left(x_{k}, r\left(1+\delta_{k}\right)\right)\right)}{\boldsymbol{\alpha}(m) r^{m}\left(1+\delta_{k}\right)^{m}}-\theta \\
& \leq\left(1+\delta_{k}\right)^{m}\left(\mathbf{c}^{*} \Xi\left(r\left(1+\delta_{k}\right)\right)+\theta\right)-\theta \\
& \rightarrow \mathbf{c}^{*} \Xi(r),
\end{aligned}
$$

for $k \rightarrow \infty$. Hence $\Theta^{m}(\|T\|, x)=\theta$ for all $x \in \operatorname{spt}(T) \cap \mathbf{U}\left(x_{0}, r_{\epsilon}\right)$ and we obtain the third conclusion. If $0<r \leq s<r_{\epsilon}$ are such that $\left(\boldsymbol{\alpha}(m) s^{m}\right)^{-1} f_{x}(s) \geq$ $\left(\boldsymbol{\alpha}(m) r^{m}\right)^{-1} f_{x}(r)$, then

$$
\begin{aligned}
\frac{\|T\|(\mathbf{B}(x, s))}{\boldsymbol{\alpha}(m) s^{m}}-\frac{\|T\|(\mathbf{B}(x, r))}{\boldsymbol{\alpha}(m) r^{m}} & =\left(\frac{\|T\|(\mathbf{B}(x, s))}{\boldsymbol{\alpha}(m) s^{m}}-\theta\right)-\left(\frac{\|T\|(\mathbf{B}(x, r))}{\boldsymbol{\alpha}(m) r^{m}}-\theta\right) \\
& \leq \mathbf{c}^{*} \Xi(s)+\mathbf{c}_{*} \Xi(r) \leq\left(\mathbf{c}^{*}+\mathbf{c}_{*}\right) \Xi(s) .
\end{aligned}
$$

Before we can apply Proposition 5.10 we need to find an open set in $\operatorname{spt}(T) \backslash$ $\operatorname{spt}(\partial T)$ where the densities are almost constant. This is possible assuming the normed Abelian group $(G,\|\cdot\|)$ is such that $\{\|g\|: g \in G\}$ is a discrete and closed subset of $\mathbb{R}$, or equivalently that

$$
\delta_{L}(G):=\inf \{|\|g\|-\|h\||: g \neq h,\|g\|,\|h\| \leq L\}>0
$$

for all $L>0$.

5.11. Lemma. Let $X$ be a Hilbert space, $(G,\|\cdot\|)$ be a normed Abelian group such that $\{\|g\|: g \in G\}$ is discrete, and $T \in \mathscr{R}_{m}(X ; G)$ be a rectifiable $G$-chain that is nearly monotonic in an open set $U \subset X \backslash \operatorname{spt}(\partial T)$. Then there is a dense open subset $U_{d}$ of $\operatorname{spt}(T) \cap U$ with the property that the map $x \mapsto \Theta^{m}(\|T\|, x)$ is locally $\|T\|$-almost constant on $U_{d}$. Moreover, if $g \mapsto\|g\|$ is constant on $G \backslash\left\{0_{G}\right\}$, then $x \mapsto \Theta^{m}(\|T\|, x)$ is $\|T\|$-almost constant on $U$.

Proof. Without loss of generality we can assume that $\|T\|(U)>0$, otherwise $\operatorname{spt}(T) \cap U=\emptyset$. It follows from Lemma 4.1 that $\Theta^{m}(\|T\|, x)$ exists for all $x \in U$ and the function $x \mapsto \Theta^{m}(\|T\|, x)$ is upper semicontinuous on $U$. Let $A$ be the subset of those $x \in U$ for which $\Theta^{m}(\|T\|, x)=\|\mathbf{g}(x)\|>0$, where $\mathbf{g}: X \rightarrow G$ is some $\mathscr{H}^{m}$-measurable $G$-orientation representing $T$. The set $A$ satisfies $\|T\|(U \backslash A)=0$ as we have seen in Subsection 2.3. For $L:=2 \cdot \operatorname{essinf}_{\|T\|}\{\|\mathbf{g}(z)\|: z \in A\}>0$ it holds that $0<\delta_{L}(G) \leq \operatorname{essinf}_{\|T\|}\{\|\mathbf{g}(z)\|: z \in A\}$ by the discreteness assumption on $G$. Now fix some point $x \in A$ with

$$
\|\mathbf{g}(x)\|<\delta_{L}(G) / 2+\operatorname{essinf}_{\|T\|}\{\|\mathbf{g}(z)\|: z \in A\} .
$$

Because $x \mapsto\|\mathbf{g}(x)\|$ is upper semicontinuous on $A$, there is some $r>0$ such that $\mathbf{B}(x, r) \subset U$ and for $\|T\|$-a.e. $y \in \mathbf{B}(x, r) \cap A$,

$$
\begin{aligned}
\operatorname{essinf}_{\|T\|}\{\|\mathbf{g}(z)\|: z \in A\} & \leq\|\mathbf{g}(y)\|<\|\mathbf{g}(x)\|+\delta_{L}(G) / 2 \\
& <\delta_{L}(G)+\operatorname{essinf}_{\|T\|}\{\|\mathbf{g}(z)\|: z \in A\} .
\end{aligned}
$$

Hence for $\|T\|$-a.e. $y \in \mathbf{B}(x, r)$,

$$
\Theta^{m}(\|T\|, y)=\|\mathbf{g}(y)\|=\operatorname{essinf}_{\|T\|}\{\|\mathbf{g}(z)\|: z \in A\} .
$$


This shows that $\Theta^{m}(\|T\|, y)$ is equal to some constant $\theta>0$ for $\|T\|$-a.e. $y \in \mathbf{B}(x, r)$. The same argument shows that for any open subset $U^{\prime} \subset U$ with $\|T\|\left(U^{\prime}\right)>0$ we can find an open subset $V^{\prime} \subset U^{\prime}$ with $\|T\|\left(V^{\prime}\right)>0$ on which $x \mapsto \Theta^{m}(\|T\|, x)$ is constant. This shows the first statement. If we further assume that $\|g\|=\theta>0$ for all $g \neq 0_{G}$, then obviously $\Theta^{m}(\|T\|, x)=\|\mathbf{g}(x)\|=\theta$ for $\|T\|$-almost every $x \in U$. Hence we can take $U_{d}=U$.

This assumption on the group is indeed a necessary one in order to obtain points of almost constant densities as we will see in Example 6.1. The following lemma is a standard result for representing sets as graphs. We say that a map $f: U \subset \mathbb{R}^{m} \rightarrow X$ is of class $C^{1, \xi}$ for some gauge $\xi$ if there is a constant $C>0$ such that for all $x, y \in U$ there is a linear map $D f_{x}: \mathbb{R}^{m} \rightarrow X$ such that

$$
\left|f(y)-f(x)-D f_{x}(y-x)\right| \leq C|x-y| \xi(C|x-y|) .
$$

5.12. Lemma. Let $S \subset X$ be a closed set with $0 \in S$ and assume that $r_{0}>0$ and $\eta:\left(0,2 r_{0}\right] \rightarrow \mathbb{R}_{+}$is a gauge with the following properties:

(1) For all $x \in \boldsymbol{B}\left(0, r_{0}\right)$ and all $0<r \leq r_{0}$ there is a plane $W_{x, r} \in \mathbf{G}(X, m)$ with

$$
d_{H}\left(S \cap \boldsymbol{B}(x, r),\left(x+W_{x, r}\right) \cap \boldsymbol{B}(x, r)\right) \leq r \eta(r) .
$$

(2) For $W:=W_{0, r_{0}}$ assume that $\pi_{W}\left(S \cap \boldsymbol{B}\left(0, \frac{r_{0}}{4}\right) \cap Z_{W}\left(0, \frac{r_{0}}{8}\right)\right)=\boldsymbol{B}_{W}\left(0, \frac{r_{0}}{8}\right)$.

(3) $\int_{0}^{2 r_{0}} \frac{\eta(r)}{r} d r \leq \frac{1}{120}$.

(4) $r \mapsto \frac{\eta(r)}{r}$ is decreasing.

Set $\hat{\eta}(r):=\int_{0}^{r} \frac{\eta(s)}{s} d s$. Then there is a unique map $f: \boldsymbol{B}_{W}\left(0, \frac{r_{0}}{8}\right) \rightarrow W_{0}^{\perp}$ with $\operatorname{graph}(f)=S \cap \boldsymbol{B}\left(0, \frac{r_{0}}{4}\right) \cap Z_{W}\left(0, \frac{r_{0}}{8}\right)$ and $f$ is of class $C^{1, \hat{\eta}}$.

Proof. Recall that for the distance on $\mathbf{G}(X, m)$ there holds $\left\|\pi_{V_{1}}-\pi_{V_{2}}\right\|=$ $d_{\mathrm{H}}\left(\mathbf{B}_{V_{1}}(0,1), \mathbf{B}_{V_{2}}(0,1)\right)$ as observed in Lemma 2.1. Since $r \mapsto \frac{\eta(r)}{r}$ is assumed to be decreasing,

$$
\hat{\eta}(r)=\int_{0}^{r} \frac{\eta(s)}{s} d s \geq \int_{0}^{r} \frac{\eta(r)}{r} d s=\eta(r) .
$$

Fix a point $x \in S \cap \mathbf{B}\left(0, r_{0}\right)$ and some $0<r \leq r_{0}$. Comparing the planes $W_{x, r}$ and $W_{x, r / 2}$ it follows from Corollary 2.4 and $\eta(r / 2) \leq \eta(r)$ that

$$
\left\|\pi_{W_{x, r}}-\pi_{W_{x, r / 2}}\right\| \leq \eta(r)\left(2+r(r / 2)^{-1}\right)=4 \eta(r) .
$$

Similarly, it follows from Lemma 2.5 and $\eta(r) \leq \eta\left(r_{0}\right) \leq \frac{1}{4}$ that for $x, y \in S \cap$ $\mathbf{B}\left(0, r_{0}\right)$ and $0<r \leq r_{0}$ with $|x-y| \leq r / 2$,

$$
\left\|\pi_{W_{x, r}}-\pi_{W_{y, r}}\right\| \leq 24 \eta(r) \text {. }
$$

To see this let $\lambda=\frac{1}{2}, \epsilon=\eta(r) \leq \frac{1}{4}$ and $\nu=4$ in the setting of Lemma 2.5. Let $r_{k}:=2^{-k} r_{0}$. It follows from (5.26) and hypothesis (4) that for $0 \leq k \leq l$,

$$
\begin{aligned}
\left\|\pi_{W_{x, r_{k}}}-\pi_{W_{x, r_{l}}}\right\| & \leq \sum_{i \geq k}\left\|\pi_{W_{x, r_{i}}}-\pi_{W_{x, r_{i+1}}}\right\| \leq 4 \sum_{i \geq k} \eta\left(r_{i}\right) \\
& =4 \sum_{i \geq k} \int_{r_{i}}^{2 r_{i}} \frac{\eta\left(r_{i}\right)}{r_{i}} \leq 4 \int_{0}^{2 r_{k}} \frac{\eta(r)}{r} d r=4 \hat{\eta}\left(2 r_{k}\right) .
\end{aligned}
$$


Because the Grassmannian $\mathbf{G}(X, m)$ is complete, there is for any $x \in S \cap \mathbf{B}\left(x_{0}, r_{0}\right)$ a plane $W_{x}=\lim _{k \rightarrow \infty} W_{x, k}$. Moreover, if $x, y \in S \cap \mathbf{B}\left(0, r_{0}\right)$ and $k \geq 1$ are such that $|x-y| \leq r_{k} / 2$, then with (5.25), (5.27) and (5.28),

$$
\begin{aligned}
\left\|\pi_{W_{x}}-\pi_{W_{y}}\right\| & \leq\left\|\pi_{W_{x, r_{k}}}-\pi_{W_{x}}\right\|+\left\|\pi_{W_{x, r_{k}}}-\pi_{W_{y, r_{k}}}\right\|+\left\|\pi_{W_{y, r_{k}}}-\pi_{W_{y}}\right\| \\
& \leq 24 \eta\left(r_{k}\right)+8 \hat{\eta}\left(2 r_{k}\right) \leq 32 \hat{\eta}\left(2 r_{k}\right) .
\end{aligned}
$$

With (5.28) and hypothesis (1),

$$
\begin{aligned}
d_{\mathrm{H}}\left(S \cap \mathbf{B}\left(x, r_{k}\right),(x\right. & \left.\left.+W_{x}\right) \cap \mathbf{B}\left(x, r_{k}\right)\right) \\
\leq & d_{\mathrm{H}}\left(S \cap \mathbf{B}\left(x, r_{k}\right),\left(x+W_{x, r_{k}}\right) \cap \mathbf{B}\left(x, r_{k}\right)\right) \\
& +d_{\mathrm{H}}\left(\left(x+W_{x, k}\right) \cap \mathbf{B}\left(x, r_{k}\right),\left(x+W_{x}\right) \cap \mathbf{B}\left(x, r_{k}\right)\right) \\
\leq & r_{k} \eta\left(r_{k}\right)+r_{k}\left\|\pi_{W_{x, r_{k}}}-\pi_{W_{x}}\right\| \\
\leq & 5 r_{k} \hat{\eta}\left(2 r_{k}\right) .
\end{aligned}
$$

Further, if $|x| \leq r_{0} / 2$, then with (5.25), (5.27), (5.28) and hypothesis (3),

$$
\begin{aligned}
\left\|\pi_{W}-\pi_{W_{x, r_{k}}}\right\| & \leq\left\|\pi_{W_{0, r_{0}}}-\pi_{W_{x, r_{0}}}\right\|+\left\|\pi_{W_{x, r_{0}}}-\pi_{W_{x, r_{k}}}\right\| \\
& \leq 24 \eta\left(r_{0}\right)+4 \hat{\eta}\left(2 r_{0}\right) \leq 30 \eta\left(2 r_{0}\right) \\
& \leq \frac{1}{4}
\end{aligned}
$$

So let $x, y \in S \cap \mathbf{B}\left(0, r_{0} / 4\right)$ and $k \geq 1$ such that $r_{k+1}<|x-y| \leq r_{k}$. By assumption there is a $v \in W_{x, r_{k}}$ with $|x+v-y| \leq r_{k} \eta\left(r_{k}\right)$. Because of (5.31),

$$
\begin{aligned}
\left|\pi_{W}(x-y)\right| & \geq\left|\pi_{W_{x, r_{k}}}(v)\right|-\left|\pi_{W_{x, r_{k}}}(x+v-y)\right|-|| \pi_{W}-\pi_{W_{x, r_{k}}}|||x-y| \\
& \geq|v|-r_{k} \eta\left(r_{k}\right)-\frac{1}{4}|x-y| \\
& \geq \frac{3}{4}|x-y|-2 r_{k} \eta\left(r_{0}\right) \\
& \geq \frac{3}{4}|x-y|-4 \hat{\eta}\left(2 r_{0}\right)|x-y| \\
& \geq \frac{1}{\sqrt{2}}|x-y| .
\end{aligned}
$$

Together with hypothesis (2) this shows that $\left.\pi_{W}: S \cap \mathbf{B}\left(0, r_{0} / 4\right) \cap Z_{W}\left(0, r_{0} / 8\right)\right) \rightarrow$ $\mathbf{B}_{W}\left(0, r_{0} / 8\right)$ is a bi-Lipschitz map. Let $f: \mathbf{B}_{W}\left(0, r_{0} / 4\right) \rightarrow W^{\perp}$ be the map that represents $\left.S \cap \mathbf{B}\left(0, r_{0} / 4\right) \cap Z_{W}\left(0, r_{0} / 8\right)\right)$ as a graph over $\mathbf{B}_{W}\left(0, r_{0} / 8\right)$. Since $\sqrt{2}\left|\pi_{W}(x-y)\right| \geq|x-y|$ for $x, y \in S \cap \mathbf{B}\left(0, r_{0} / 4\right)$, we can estimate the Lipschitz constant by $\operatorname{Lip}(f) \leq 1$. (5.31) also implies that for $v \in W_{x}$,

$$
\left|\pi_{W}(v)\right| \geq|v|-\frac{1}{4}|v| \geq \frac{1}{\sqrt{2}}|v| .
$$

This shows that $\pi_{W}: W_{x} \rightarrow W$ is injective and there is a linear map $L_{x}: W \rightarrow W^{\perp}$ with $\left\|L_{x}\right\| \leq 1$ which represents $W_{x}$ as a graph over $W$.

Let $w, w^{\prime} \in \mathbf{B}_{W}\left(0, r_{0} / 8\right)$ with $r_{k+1}<\left|w-w^{\prime}\right| \leq r_{k}$ for some $k \geq 2$ and set $x:=w+f(w), x^{\prime}:=w^{\prime}+f\left(w^{\prime}\right) \in S$. Since $f$ is 1-Lipschitz,

$$
r_{k+1} \leq\left|w-w^{\prime}\right| \leq\left|x-x^{\prime}\right| \leq\left|w-w^{\prime}\right|+\left|f(w)-f\left(w^{\prime}\right)\right| \leq 2\left|w-w^{\prime}\right| \leq r_{k-1} .
$$


In particular $x, x^{\prime} \in S \cap \mathbf{B}\left(0, r_{0} / 4\right)$ and by (5.30) there is some $v \in W_{x}$ with $\left|x+v-x^{\prime}\right| \leq 5 r_{k-1} \hat{\eta}\left(2 r_{k-1}\right)$. Because $\pi_{W^{\perp}}(v)=L_{x}\left(\pi_{W}(v)\right)$, we conclude

$$
\begin{aligned}
\left|f\left(w^{\prime}\right)-f(w)-L_{x}\left(w^{\prime}-w\right)\right| & \leq\left|f\left(w^{\prime}\right)-f(w)-\pi_{W^{\perp}}(v)\right|+\left|L_{x}\left(\pi_{W}(v)+w-w^{\prime}\right)\right| \\
& =\left|\pi_{W^{\perp}}\left(x^{\prime}-x-v\right)\right|+\left|L_{x}\left(\pi_{W}\left(v+x-x^{\prime}\right)\right)\right| \\
& \leq 2\left|x^{\prime}-x-v\right| \leq 10 r_{k-1} \hat{\eta}\left(2 r_{k-1}\right) \\
& \leq 40\left|w-w^{\prime}\right| \hat{\eta}\left(8\left|w-w^{\prime}\right|\right) .
\end{aligned}
$$

This shows the result.

Note that if $\eta(r)=c r^{\alpha}$ for some $0<\alpha \leq 1$, then $\hat{\eta}(r)=\frac{c}{\alpha} r^{\alpha}$ and hypothesis (4) is satisfied because of Lemma 5.6(2). Combining Proposition 5.10, Lemma 5.11 and Lemma 5.12 we obtain our main regularity result.

5.13. Theorem. Let $X$ be a Hilbert space with $\operatorname{dim}(X)>m \geq 1$ and let $(G,\|\cdot\|)$ be a normed Abelian group such that $\{\|g\|: g \in G\}$ is discrete. There is a constant $0<\alpha_{0}<1$ with the following property. Let $T \in \mathscr{R}_{m}(X ; G)$ and $U \subset X \backslash \operatorname{spt}(T)$ be an open set. Assume that $T$ is $(\mathbf{M}, \xi, \delta)$-minimal in $U$ for some gauge $\xi(r)=c r^{\alpha}$, where $\alpha>0$. Then there is an open dense subset $U_{\text {reg }}$ of $\operatorname{spt}(T) \cap U$ that is a $C^{1, \beta}$-submanifold of $X$ with

$$
\beta:=\frac{\min \left\{\alpha_{0}, \alpha\right\}}{8(m+2)} .
$$

Moreover, if $g \mapsto\|g\|$ is constant on $G \backslash\left\{0_{G}\right\}$, then

$$
\mathscr{H}^{m}\left(\operatorname{spt}(T) \cap U \backslash U_{\text {reg }}\right)=\|T\|\left(U \backslash U_{\text {reg }}\right)=0 .
$$

Proof. We can assume that $\delta \leq 1$, and hence $T$ is almost minimal with respect to the gauge $r \mapsto c r^{\min \left\{\alpha_{0}, \alpha\right\}}$, where $\alpha_{0}:=m \frac{\sqrt[1-\sqrt[4]{\lambda_{3} .18}]{\sqrt[4]{\lambda} 3.18}}{\sqrt{\lambda_{1}}}<1$. By restricting to the smaller of the two values we can further assume that $\alpha \leq \alpha_{0}$. As before we define $\Xi(r):=m \int_{0}^{r} \frac{\xi(s)}{s} d s=\frac{m}{\alpha} r^{\alpha}$.

Without loss of generality we can assume that $\operatorname{spt}(T) \cap U \neq \emptyset$ and by exhaustion we may also assume that $\operatorname{dist}(U, \operatorname{spt}(\partial T))>0$. Due to Lemma 5.2 and Lemma 4.2, $T$ is nearly monotonic in $U$. Because of Lemma 5.11 there is a dense open set $U_{d}$ of $\operatorname{spt}(T) \cap U$ that has locally $\|T\|$-almost constant densities. With Proposition 5.10 and Lemma 5.6 we deduce that for $\|T\|$-a.e. $x_{0} \in U_{d}$ there are $c^{\prime}, r_{1}>0$ such that

$$
\begin{aligned}
\mathbf{e x c}_{*}^{m}(\|T\|, x, r) \leq c^{\prime} r^{\alpha}, \text { if } x \in \mathbf{U}\left(x_{0}, r_{1}\right), \\
\operatorname{exc}^{m *}(\|T\|, x, r) \leq c^{\prime} r^{\alpha}, \text { if } x \in \operatorname{spt}(T) \cap \mathbf{U}\left(x_{0}, r_{1}\right) .
\end{aligned}
$$

The assumption $\alpha \leq \alpha_{0}$ is needed for Proposition 5.10. With Theorem 5.9 we can further assume that $r_{1}$ is small enough such that we have orthogonal frames in the support of $T$ around all points $x \in \operatorname{spt}(T) \cap \mathbf{U}\left(x_{0}, r_{1}\right)$ and for all scales $0<r<r_{1}$. Let us assume that $\Theta^{m}(\|T\|, x)=\theta>0$ for $\|T\|$-a.e. $x \in \mathbf{U}\left(x_{0}, r_{1}\right)$. Applying the moments computations we obtain from Proposition 4.14 that there is a scale $0<r_{2} \leq r_{1}$ and $c^{\prime \prime}>0$ such that for all $x \in \operatorname{spt}(T) \cap \mathbf{U}\left(x_{0}, r_{2}\right)$ with $\Theta^{m}(\|T\|, x)=\theta$ and all $0<r<r_{2}$ there is a plane $W \in \mathbf{G}(X, m)$ with

$$
\begin{aligned}
\beta_{\infty}(\|T\|, x, r, W) & <\mathbf{c}_{4.14} \max \{\sqrt[8]{2 r}, \sqrt[4]{\Xi(2 \sqrt{2 r})}\}^{\frac{1}{m+2}} \\
& \leq c^{\prime \prime} r^{\beta},
\end{aligned}
$$


and further if $r_{2}$ is small enough, Theorem 5.9 and Lemma 2.7 imply that

$$
\pi_{W \#}\left(T \left\llcorner\left(\mathbf{B}(x, r) \cap Z_{W}\left(\pi_{W}(x), \frac{r}{2}\right)\right)=g_{x} \llbracket \mathbf{B}_{W}\left(\pi_{W}(x), \frac{r}{2}\right) \rrbracket,\right.\right.
$$

for some $g_{x} \in G$ with $\left\|g_{x}\right\|=\theta$. As in the last part of the proof of Lemma 5.8 we obtain that

$$
d_{\mathrm{H}}(\operatorname{spt}(T) \cap \mathbf{B}(x, r),(x+W) \cap \mathbf{B}(x, r))<2 c^{\prime \prime} r^{1+\beta} .
$$

These points $x$ are dense $\operatorname{spt}(T) \cap \mathbf{U}\left(x_{0}, r_{2}\right)$ and as in the proof of Theorem 5.9 we conclude that for all $x \in \operatorname{spt}(T) \cap \mathbf{U}\left(x_{0}, r_{2}\right)$ and $0<r<r_{2}$ there is a plane with (5.32) and (5.33). If $W$ is such a plane at $x_{0}$ and scale $0<r<r_{2}$, the constancy theorem implies further that that $\pi_{W}: \operatorname{spt}(T) \cap \mathbf{B}\left(x_{0}, r\right) \cap Z_{W}\left(\pi_{W}\left(x_{0}\right), \frac{r}{2}\right) \rightarrow$ $\mathbf{B}_{W}\left(\pi_{W}\left(x_{0}\right), \frac{r}{2}\right)$ is surjective. This allows to apply Lemma 5.12 and we see that $\operatorname{spt}(T) \cap \mathbf{U}\left(x_{0}, r\right)$ is a $C^{1, \beta}$-submanifold of $X$ if $r$ is small enough. Let $U_{\text {reg }}$ be the set of points in $\operatorname{spt}(T) \cap U$ with a relatively open neighborhood which is a $C^{1, \beta}$-submanifold of $X$. The observations above show that any $x_{0} \in U_{d}$ with $\Theta^{m}\left(\|T\|, x_{0}\right)=\theta$ is contained in $U_{d}$. Hence $\|T\|\left(U_{d} \backslash U_{\text {reg }}\right)=0$ and since $\operatorname{spt}(T) \cap U_{d}$ is dense in $\operatorname{spt}(T) \cap U, U_{\text {reg }}$ is dense in $\operatorname{spt}(T) \cap U$. In case $g \mapsto\|g\|=\theta$ is constant on $G \backslash\left\{0_{G}\right\}$, the set of points $x_{0} \in \operatorname{spt}(T) \cap U_{d}$ with $\Theta^{m}\left(\|T\|, x_{0}\right)=\theta$ forms a set of full $\|T\|$-measure, hence $\|T\|\left(X \backslash U_{\text {reg }}\right)=0$ because $\|T\|\left(X \backslash U_{d}\right)=0$.

This theorem can be formulated for other gauge functions $\xi$. If we assume that $\xi$ is a continuous gauge such that:

(1) $r \mapsto \frac{\xi(r)}{r^{\alpha}}$ is decreasing, where $\alpha \leq \alpha_{0}:=\min \left\{m \frac{1-\sqrt[4]{\lambda_{3} .18}}{\sqrt[4]{\lambda^{\lambda} 3.18}}, \frac{1}{8(m+2)}\right\}$;

(2) $\Xi(r):=m \int_{0}^{r} \frac{\xi(s)}{s} d s<\infty$ for all $r$;

(3) $\hat{\eta}(r):=\int_{0}^{r} \frac{\eta(s)}{s} d s<\infty$ for all $r$ where $\eta(r):=\Xi(2 \sqrt{r})^{\frac{1}{4(m+2)}}$.

Under the same assumptions as in the theorem above we obtain a $C^{1, \hat{\eta}}$ regularity result.

By hypothesis (1) and Lemma 5.6 there is some constant $c>0$ such that

$$
\Xi(r) \geq m \xi(r) \geq \xi(r) \geq c r^{\frac{1}{8(m+2)}},
$$

for all small $r>0$. Hence

$$
\eta(r) \geq \max \{1, c\} \max \{\sqrt[8]{r}, \sqrt[4]{\Xi(2 \sqrt{r})}\}^{\frac{1}{m+2}} .
$$

In order to establish the technical assumption (4) in Lemma 5.12, note that,

$$
\begin{aligned}
\partial_{r} \frac{\eta(r)}{r} & =-\frac{1}{r^{2}} \Xi(2 \sqrt{r})^{\frac{1}{4(m+2)}}+\frac{1}{r} \frac{1}{4(m+2)} \Xi(2 \sqrt{r})^{\frac{1}{4(m+2)}-1} \frac{m}{2 \sqrt{r}} \xi(2 \sqrt{r}) \frac{1}{\sqrt{r}} \\
& \leq-\frac{m^{\frac{1}{4(m+2)}}}{r^{2}} \xi(2 \sqrt{r})^{\frac{1}{4(m+2)}}+\frac{1}{r^{2}} \frac{m^{\frac{1}{4(m+2)}}}{4(m+2)} \xi(2 \sqrt{r})^{\frac{1}{4(m+2)}-1} \xi(2 \sqrt{r}) \\
& =\frac{m^{\frac{1}{4(m+2)}}}{r^{2}} \xi(2 \sqrt{r})^{\frac{1}{4(m+2)}}\left(\frac{1}{4(m+2)}-1\right) \leq 0 .
\end{aligned}
$$

This shows that $r \mapsto \frac{\eta(r)}{r}$ is decreasing and with the help of Lemma 5.5, Lemma 5.6 and Lemma 5.12, the regularity result follows as in the proof of Theorem 5.13. 


\section{Examples And Counterexamples}

In this section we give examples indicating the sharpness of our results. In the example below we show that in case $\{\|g\|: g \in G\}$ is not discrete, there is in general no uniform lower density bound and mass minimizing $G$-chains may have no point of regularity.

6.1. Example (Discreteness of the group). Let $G=(\mathbb{Z} / 2 \mathbb{Z})^{\infty}$ be the Abelian group with coordinatewise addition in $\mathbb{Z} / 2 \mathbb{Z}$ and norm

$$
\left\|\left(a_{1}, a_{2}, a_{3}, \ldots\right)\right\|:=\sum_{i=1}^{\infty} \frac{1}{3^{i}}\left|a_{i}\right|,
$$

where $\left|1_{\mathbb{Z} / 2 \mathbb{Z}}\right|=1$ and $\left|0_{\mathbb{Z} / 2 \mathbb{Z}}\right|=0$. As a metric space $(G,\|\cdot\|)$ is bi-Lipschitz equivalent to the standard Cantor set and therefore totally disconnected but not discrete. Let $g_{i} \in G$ be the sequence $\left(a_{1}, a_{2}, a_{3}, \ldots\right)$ with $a_{i}=1$ and $a_{j}=0$ for $j \neq i$. Let $\left\{p_{i}\right\}_{i \geq 1} \subset[0,1]$ be a countable dense subset and consider the $G$-chain

$$
T=\sum_{i} g_{i} \llbracket\left(0, p_{i}\right),\left(1, p_{i}\right) \rrbracket \in \mathscr{R}_{1}\left(\mathbb{R}^{2} ; G\right) .
$$

Let $\pi(x, y)=x$ be the projection onto the first coordinate. A straightforward calculation shows that for $g=(1,1, \ldots)$,

$$
\begin{aligned}
\mathbf{M}\left(\pi_{\#} T\right) & =\mathbf{M}(g \llbracket(0,0),(1,0) \rrbracket)=\|g\|=\sum_{i \geq 1}\left\|g_{i}\right\| \\
& =\sum_{i \geq 1} \mathbf{M}\left(g_{i} \llbracket\left(0, p_{i}\right),\left(1, p_{i}\right) \rrbracket\right)=\mathbf{M}(T) .
\end{aligned}
$$

The constancy theorem implies that for any filling $S \in \mathscr{R}_{1}\left(\mathbb{R}^{2} ; G\right)$ of $\partial T$ there holds $\pi_{\#} S=g \llbracket(0,0),(1,0) \rrbracket$ and since $\pi$ is 1 -Lipschitz,

$$
\mathbf{M}(T)=\mathbf{M}\left(\pi_{\#} T\right)=\mathbf{M}\left(\pi_{\#} S\right) \leq \mathbf{M}(S) .
$$

Thus $T$ is a mass minimizing filling of $\partial T$, but $\operatorname{spt}(T)=[0,1]^{2}$ is not $\mathscr{H}^{1}$-rectifiable and in particular contains no point of regularity and no lower bound on 1-densities.

Under suitable conditions, the set of regular points of an almost minimizing chain is a $C^{1}$ submanifold. The converse is true too, even allowing for nice unions of such submanifolds as we show in the proposition below. Let $M \subset X$ be an oriented $m$ dimensional submanifold of regularity $C^{1}$ possibly with boundary. At any $x \in M$ there is a tangent plane $\operatorname{Tan}(M, x) \in \mathbf{G}(X, m)$. Since $M$ is oriented, there is a unique choice of orientation for $\operatorname{Tan}(M, x)$ such that $\pi_{\operatorname{Tan}(M, x)}: M \cap \mathbf{B}(0, r) \rightarrow$ $\operatorname{Tan}(M, x)$ is an orientation preserving homeomorphism onto its image for all small enough $r$.

6.2. Proposition. Let $0<\alpha \leq 1$ and $M_{1}, \ldots, M_{k} \subset X$ be compact oriented $m$ dimensional submanifolds in $X$ of regularity $C^{1, \alpha}$. Let $T:=\llbracket M_{1} \rrbracket+\cdots+\llbracket M_{k} \rrbracket \in$ $\mathscr{R}_{m}(X ; \mathbb{Z})$. Further assume that there are $C, \delta>0$ such that:

(1) If $x \in M_{i}$ and $y \in M_{j}$ for some $i, j$, then

$$
\left\|\pi_{\operatorname{Tan}\left(M_{i}, x\right)}-\pi_{\operatorname{Tan}\left(M_{j}, y\right)}\right\| \leq C|x-y|^{\alpha} .
$$

(2) If $x \in M_{i}, y \in M_{j}$ and $|x-y| \leq \delta$ for some $i, j$, then the orthogonal projection from $\operatorname{Tan}\left(M_{j}, y\right)$ to $\operatorname{Tan}\left(M_{i}, x\right)$ is orientation preserving. 
Then there are constants $C^{\prime}, \delta^{\prime}>0$ such that $T$ is $\left(\mathbf{M}, \xi, \delta^{\prime}\right)$-minimal with $\xi(t):=$ $C^{\prime} t^{2 \alpha}$.

Note that if we consider only one submanifold, then these two conditions are trivially satisfied. See [11, Lemma 2.2.3] for two different characterizations of $C^{1, \alpha}$ manifolds in $\mathbb{R}^{n}$ which easily generalize to Hilbert spaces.

Proof. Since all the $M_{i}$ are Hölder regular and compact there are constants $C_{1}, \delta_{1}>$ 0 with the following property: If $x \in M_{i} \backslash \partial M_{i}$ for some $i$ and $W \in \mathbf{G}(X, m)$ is an $m$ plane with $d_{W, i, x}:=\left\|\pi_{\operatorname{Tan}\left(M_{i}, x\right)}-\pi_{W}\right\| \leq \delta_{1}$, then

(1) for all $r>0$ with $C r^{\alpha} \leq \delta_{1}$, the orthogonal projection $\pi_{W}: M_{i} \cap \mathbf{B}(x, r) \rightarrow$ $B_{W, i, x, r} \subset W$ is a homeomorphism onto a neighborhood $B_{W, i, x, r}$ of $\pi_{W}(x)$ in $W$.

(2) If $\mathbf{w}: B_{W, i, x, r} \rightarrow W^{\perp}$ is the map with the defining property $w+\mathbf{w}(w) \in M$ in case $w \in U_{W, i, x, r}$, then

$$
\sup _{w \in B_{W, i, x, r}}\left\|D \mathbf{w}_{w}\right\| \leq C_{1}\left(d_{W, i, x}+r^{\alpha}\right) .
$$

For (2) compare with the proof of Lemma 5.12 or [11, Lemma 2.2.3]. If we assume that $W$ satisfies $d_{W, i, x}=\left\|\pi_{\operatorname{Tan}\left(M_{i}, x\right)}-\pi_{W}\right\| \leq C r^{\alpha} \leq \delta_{1}$, then there is a constant $C_{2}>0$ such that for all $w \in B_{W, i, x, r}$

$$
\left(\mathcal{J} \mathbf{w}_{w}\right)^{2}=\left\|D \mathbf{w}_{w}\right\|_{\mathrm{HS}}^{2}+\sum_{\# K \geq 2} \operatorname{det}\left(\left(D \mathbf{w}_{w}^{*} D \mathbf{w}_{w}\right)_{K}\right) \leq C_{2} r^{2 \alpha} .
$$

See (2.5) and (2.6). Hence

$$
\left(1+\left(\mathcal{J} \mathbf{w}_{w}\right)^{2}\right)^{\frac{1}{2}} \leq\left(1+C_{2} r^{2 \alpha}\right)^{\frac{1}{2}} \leq 1+C_{2} r^{2 \alpha} .
$$

Because $\pi_{W}: M_{i} \cap \mathbf{B}(x, r) \rightarrow B_{W, i, x, r} \subset W$ is a homeomorphism by (1), the cylindrical excess of a compact subset $A \subset M_{i} \cap \mathbf{B}(x, r)$ over the plane $W$ estimates as

$$
\begin{aligned}
0 \leq \mathscr{H}^{m}(A)-\mathscr{H}^{m}\left(\pi_{W}(A)\right) & =\int_{\pi_{W}(A)}\left(1+\left(\mathcal{J} \mathbf{w}_{w}\right)^{2}\right)^{\frac{1}{2}}-1 d w \\
& \leq C_{2} \int_{\pi_{W}(A)} r^{2 \alpha} d w \\
& =C_{2} r^{2 \alpha} \mathscr{H}^{m}\left(\pi_{W}(A)\right) .
\end{aligned}
$$

Let $0<\delta_{2} \leq \frac{1}{2} \delta$ be small enough such that $C\left(2 \delta_{2}\right)^{\alpha} \leq \delta_{1}$ and consider some scale $0<r \leq \delta_{2}$ and some $x \in X$. Note that by the compatibility of orientations, $\operatorname{spt}(\partial T)=\partial M_{1} \cup \cdots \cup \partial M_{k}$. Assume that $\mathbf{B}(x, r)$ intersects $M_{i(1)}, \ldots, M_{i(l)}$ in a set of positive measure and fix some points $x_{j} \in A_{j}:=M_{i(j)} \cap \mathbf{B}(x, r) \backslash \partial M_{i(j)}$ as well as $W:=\operatorname{Tan}\left(M_{i(1)}, x_{1}\right)$. Since $2 r \leq \delta$, for all $j=1, \ldots, l$ there holds,

$$
\left\|\pi_{\operatorname{Tan}\left(M_{i(j)}, x_{j}\right)}-\pi_{W}\right\| \leq C\left|x_{1}-x_{j}\right|^{\alpha} \leq C(2 r)^{\alpha} \leq \delta_{2} .
$$

Because $A_{j} \subset M_{i(j)} \cap \mathbf{B}\left(x_{j}, 2 r\right)$ it follows from (6.1) that

$$
\mathscr{H}^{m}\left(A_{j}\right) \leq\left(1+C_{2}(2 r)^{2 \alpha}\right) \mathscr{H}^{m}\left(\pi_{W}\left(A_{j}\right)\right)=\left(1+C_{3} r^{2 \alpha}\right) \mathscr{H}^{m}\left(\pi_{W}\left(A_{j}\right)\right),
$$

for $C_{3}:=4 C_{2}$. With the hypothesis on the orientation and relative position of the submanifolds, $\mathbf{M}(T)=\mathbf{M}\left(\llbracket M_{1} \rrbracket\right)+\cdots+\mathbf{M}\left(\llbracket M_{k} \rrbracket\right)$. More precisely, at every point where two submanifolds meet, they have the same tangent plane with compatible 
orientations and thus their multiplicities do not cancel. Since all the projections $\pi_{W}: A_{j} \rightarrow W$ are injective and orientation preserving,

$$
\begin{aligned}
& \mathbf{M}\left(T\llcorner\mathbf{B}(x, r))=\mathbf{M}\left(\llbracket A_{1} \rrbracket\right)+\cdots+\mathbf{M}\left(\llbracket A_{l} \rrbracket\right)\right. \\
& \leq\left(1+C_{3} r^{2 \alpha}\right) \mathbf{M}\left(\pi_{W \# \llbracket} \llbracket A_{1} \rrbracket\right)+\cdots+\left(1+C_{3} r^{2 \alpha}\right) \mathbf{M}\left(\pi_{W \# \llbracket} \llbracket A_{l} \rrbracket\right) \\
& =\left(1+C_{3} r^{2 \alpha}\right) \mathbf{M}\left(\pi_{W \#}(T\llcorner\mathbf{B}(x, r)))\right. \text {. }
\end{aligned}
$$

Let $S \in \mathscr{R}_{m}(X ; \mathbb{Z})$ with $\operatorname{spt}(S) \subset \mathbf{B}(x, r)$ and $\partial S=0$. The constancy theorem implies $\pi_{W \#} S=0$ and hence

$$
\begin{aligned}
\mathbf{M}(T\llcorner\mathbf{B}(x, r)) & \leq\left(1+C_{3} r^{2 \alpha}\right) \mathbf{M}\left(\pi_{W \#}(T\llcorner\mathbf{B}(x, r)))\right. \\
& =\left(1+C_{3} r^{2 \alpha}\right) \mathbf{M}\left(\pi_{W \#}(T\llcorner\mathbf{B}(x, r)+S))\right. \\
& \leq\left(1+C_{3} r^{2 \alpha}\right) \mathbf{M}(T\llcorner\mathbf{B}(x, r)+S) .
\end{aligned}
$$

This shows that $T$ is almost minimizing.

The following example illustrates the proposition above showing that if $G=\mathbb{Z}$, then the set of regular points of an almost minimizing rectifiable $G$-chain is in general not of full measure. The same construction also works for an arbitrary normed Abelian group $G$ if there are $g_{1}, g_{2} \in G \backslash\left\{0_{G}\right\}$ with $\left\|g_{1}\right\|+\left\|g_{2}\right\|=\left\|g_{1}+g_{2}\right\|$.

6.3. Example (Size of the regular set). Let $C \subset[0,1]$ be a topological Cantor set with $\mathscr{H}^{1}(C)>0$ and $\{0,1\} \subset C$. Let $\gamma: \mathbb{R} \rightarrow \mathbb{R}$ be a smooth function with $\gamma(t)>0$ for $|t|<1$ and $\gamma(t)=0$ for $|t| \geq 1$. We could for example take

$$
\gamma(t):= \begin{cases}\exp \left(-1 /\left(1-t^{2}\right)\right) & \text { if }|t|<1 \\ 0 & \text { if }|t| \geq 1\end{cases}
$$

Fix an enumeration $U_{i}$ of the connected components of $[0,1] \backslash C$. Since $\{0,1\} \subset C$ each $U_{i}$ is of the form $\left(a_{i}-b_{i}, a_{i}+b_{i}\right)$ for some $0<a_{i}, b_{i}<1$. We define $\gamma_{i}: \mathbb{R} \rightarrow \mathbb{R}$ by $\gamma_{i}(t):=c_{i} \gamma\left(b_{i}^{-1}\left(t-a_{i}\right)\right)$ for some $c_{i}>0$ such that $\left|D^{k} \gamma_{i}\right| \leq 2^{-i}$ for all $k \leq i$. Set $f: \mathbb{R} \rightarrow \mathbb{R}$ to be the sum $f:=\sum_{i} \gamma_{i}$. Then the partial sums $\sum_{i} D^{k} \gamma_{i}$ converge uniformly for all $k$ and hence $f$ is smooth and satisfies $f(t)=0$ for $t \in C$ and $f(t)>0$ for $t \in[0,1] \backslash C$.

Consider

$$
T:=\left(\operatorname{id}_{\mathbb{R}} \times 0\right)_{\#} \llbracket 0,1 \rrbracket+\left(\operatorname{id}_{\mathbb{R}} \times f\right)_{\#} \llbracket 0,1 \rrbracket \in \mathscr{R}_{1}\left(\mathbb{R}^{2} ; \mathbb{Z}\right) .
$$

Clearly, $\partial T=\left(g_{1}+g_{2}\right)(\llbracket 1 \rrbracket-\llbracket 0 \rrbracket)$ and the set $C$ is the complement of the set of regular points. Since $\|T\|(C)=2 \mathscr{H}^{1}(C)>0$, the set of regular points doesn't have full measure. It remains to check that $T$ is almost minimizing. Since $f \in C^{2}$ has compact support, there is a constant $L>0$ such that for all $x, y \in \mathbb{R}$,

$$
\left|f(y)-f(x)-f^{\prime}(x)(y-x)\right| \leq L|y-x|^{2} .
$$

Assume that $f^{\prime}(x)^{2}>4 L f(x)$ for some $\epsilon>0$. If $f^{\prime}(x)>0$ let $y \in \mathbb{R}$ be such that $x-y=f^{\prime}(x)(2 L)^{-1}$, and $y-x=f^{\prime}(x)(2 L)^{-1}$ otherwise. We get

$$
\begin{aligned}
f(y) & \leq f(x)+f^{\prime}(x)(y-x)+L|y-x|^{2} \\
& <f^{\prime}(x)(4 L)^{-1}-f^{\prime}(x)^{2}(2 L)^{-1}+f^{\prime}(x)^{2}(4 L)^{-1} \\
& =0 .
\end{aligned}
$$


This contradicts $f(y) \geq 0$ and hence $f^{\prime}(x)^{2} \leq 4 L f(x)$ for all $x \in[0,1] \backslash C$. Let $W_{1}=\left\{\left(t, t f^{\prime}(x)\right): t \in \mathbb{R}\right\}$ be the tangent line at $(x, f(x))$ to $T$ and $W_{0}=\{(t, 0)$ : $t \in \mathbb{R}\}$ the tangent line to $T$ at $(x, 0)$. A simple calculation shows that

$$
\left\|\pi_{W_{0}}-\pi_{W_{1}}\right\| \leq f^{\prime}(x) \leq(4 L)^{\frac{1}{2}} f(x)^{\frac{1}{2}},
$$

and hence for all $p, q \in \operatorname{spt}(T)$,

$$
\left\|\pi_{\operatorname{Tan}(T, p)}-\pi_{\operatorname{Tan}(T, q)}\right\| \leq L^{\prime}|p-q|^{\frac{1}{2}},
$$

for some $L^{\prime}>0$. Proposition 6.2 now shows that $T$ is almost minimizing with a linear gauge $\xi(r)=L_{4} r$. A more careful analysis shows that $T$ is actually almost minimizing with respect to a quadratic gauge function. But since Theorem 5.13 certainly applies to a linear gauge, this is enough to show that almost minimizing chains can have a branching set of positive measure.

\section{REFERENCES}

[1] F. J. Almgren, Existence and regularity almost everywhere of solutions to elliptic variational problemsamong surfaces of varying topological type and singularity structure, Ann. of Math. 87, 1968, 321-391.

[2] F. J. Almgren, Existence and regularity almost everywhere of solutions to elliptic variational problems with constraints, Mem. Amer. Math. Soc. No. 165, 1976.

[3] F. J. Almgren, Almgrens big regularity paper, volume 1 of World Scientific Monograph Series in Mathematics, World Scientific Publishing Co. Inc., 2000.

[4] L. Ambrosio, C. De Lellis and T. Schmidt, Partial regularity for mass-minimizing currents in Hilbert spaces, J. Reine Angew. Math. 734, 2018, 99-144.

[5] L. Ambrosio and B. Kirchheim, Rectifiable sets in metric and Banach spaces, Math. Ann. 318(3), 2000, 527-555.

[6] L. Ambrosio and B. Kirchheim, Currents in metric spaces, Acta Math. 185(1), 2000, 1-80.

[7] L. Ambrosio and P. Tilli, Topics on analysis in metric spaces, Oxford University Press, 2004.

[8] E. Bombieri, Regularity theory for almost minimal currents, Arch. Ration. Mech. Anal. 78(2), 1982, 99-130.

[9] E. De Giorgi, Frontiere orientate di misura minima, Seminario Mat. Scuola Norm. Sup. Pisa, 1961.

[10] Th. De Pauw, Approximation by polyhedral G chains in Banach spaces, Z. Anal. Anwend. 33, 2014. 311-334.

[11] Th. De Pauw, Concentrated, nearly monotonic, epiperimetric measures in Euclidean space, J. Differential Geom. 77(1), 2007, 77-134.

[12] Th. De Pauw, An example pertaining to the failure of the Besicovitch-Federer Theorem in separable Hilbert space, Publ. Mat. 61(1), 2017, 153-173.

[13] Th. De Pauw and R. Hardt, Rectifiable and flat G chains in metric spaces, Amer. J. Math. 134(1), 2012, 1-69.

[14] Th. De Pauw and R. Hardt, Some basic theorems on flat G chains, J. Math. Anal. Appl. 418, 2014, 1047-1061.

[15] H. Federer, Geometric measure theory, Springer-Verlag, 1969.

[16] B. Kirchheim, Rectifiable metric spaces: local structure and regularity of the Hausdorff measure, Proceedings of the American Mathematical Society 121(1), 1994, 113-123.

[17] C. B. Morrey Jr., Multiple integrals in the calculus of variations, Springer, 1966.

[18] D. Preiss, Geometry of measures in $\mathbb{R}^{n}$ : distribution, rectifiability, and densities, Ann. Math. 125(3), 1987, 537-643.

[19] E. R. Reifenberg, Solution of the Plateau problem for m-dimensional surfaces of varying topological type, Acta Math. 104, 1960, 1-92.

[20] E. R. Reifenberg, An epiperimetric inequality related to the analyticity of minimal surfaces, Annals of Mathematics 80(1), 1964, 1-14.

[21] E. R. Reifenberg, On the analyticity of minimal surfaces, Ann. of Math. 80, 1964, 15-21.

[22] J. E. Taylor, The structure of singularities in soap-bubble-like and soap-film-like minimal surfaces, Annals of Mathematics. Second Series 103(3), 1976, 489-539. 
School of Mathematical Sciences, Shanghai Key laboratory of PMMP, East China Normal University, 500 Dongchuang Road, Shanghai 200062, P.R. of China, and NyUECNU Institute of Mathematical Sciences at NYU Shanghai, 3663 Zhongshan Road North, Shanghai 200062, China

On leave of absence from: Université Paris Diderot, Sorbonne Université, CNRS, Institut de Mathématiques de Jussieu - Paris Rive Gauche, IMJ-PRG, F-75013, Paris, France

E-mail address: thdepauw@math.ecnu.edu.cn,thierry.de-pauw@imj-prg.fr

University of Bern, Mathematical Institute, Alpeneggstrasse 22, 3012 Bern, SWITZERLAND

E-mail address: roger.zuest@math.unibe.ch 